\title{
NUREG/CR-1133 Appendix
}

ANL/ES-81 Appendix

NUREG/CR-1133 Appendix

ANL/ES-81 Appendix

\section{APPENDIX TO \\ RADON AND RADON-DAUGHTER CONCENTRATIONS \\ IN AIR IN THE VICINITY OF \\ THE ANACONDA URANIUM MILL}

by

Michael H. Momeni, J. B. Lindstrom,

C. E. Dungey, and Walter E. Kisieleski
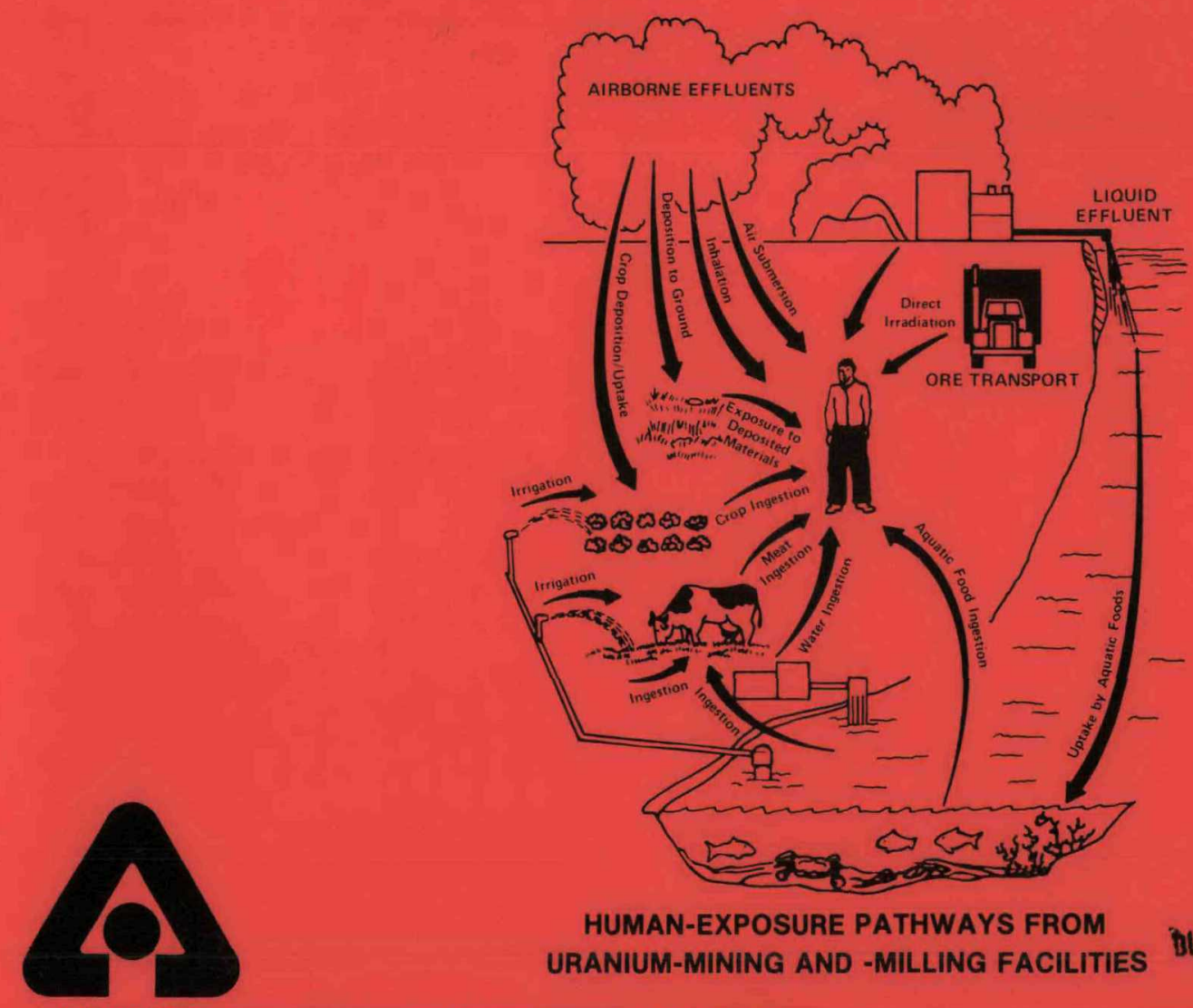

HUMAN-EXPOSURE PATHWAYS FROM URANIUM-MINING AND -MILLING FACILITIES

BISTRIBUTION OF THIS DOCUMENT IS UNCIMITED

UOFC-AUA-USDOE

ARGONNE NATIONAL LABORATORY, ARGONNE, ILLINOIS

Prepared for the U. S. NUCLEAR REGULATORY COMMISSION under Interagency Agreement DOE 40-550-75 
The facilities of Argonne National Laboratory are owned by the United States Government. Under the terms of a contract (W-31-109-Eng-38) among the U.S. Department of Energy, Argonne Universities Association and The University of Chicago, the University employs the staff and operates the Laboratory in accordance with policies and programs formulated, approved and reviewed by the Association.

\section{MEMBERS OF ARGONNE UNIVERSITIES ASSOCIATION}

The University of Arizona Carnegie-Mellon University Case Western Reserve University The University of Chicago University of Cincinnati Illinois Institute of Technology University of Illinois Indiana University The University of Iowa Iowa State University
The University of Kansas Kansas State University Loyola University of Chicago Marquette University The University of Michigan Michigan State University University of Minne sota University of Missouri Northwe stern University University of Notre Dame
The Ohio State University Ohio University

The Pennsylvania State University Purdue University

Saint Louis University Southe rn Illinois University

The University of Texas at Austin

Washington University

Wayne State University

The University of Wisconsin-Madison

\section{NOTICE}

This report was prepared as an account of work sponsored by an agency of the United States Government. Neither the United States Government nor any agency thereof, nor any of their contractors, subcontractors, or any of their employees, makes any warranty, expressed or implied, or assumes any legal liability or responsibility for any third party's use, or the results of such use, of any information, apparatus, product or process disclosed in this report, or represents that its use by such third party would not infringe privately-owned rights.

Available from

National Technical Information Service

Springfield, Virginia 22161 


\section{DISCLAIMER}

This report was prepared as an account of work sponsored by an agency of the United States Government. Neither the United States Government nor any agency Thereof, nor any of their employees, makes any warranty, express or implied, or assumes any legal liability or responsibility for the accuracy, completeness, or usefulness of any information, apparatus, product, or process disclosed, or represents that its use would not infringe privately owned rights. Reference herein to any specific commercial product, process, or service by trade name, trademark, manufacturer, or otherwise does not necessarily constitute or imply its endorsement, recommendation, or favoring by the United States Government or any agency thereof. The views and opinions of authors expressed herein do not necessarily state or reflect those of the United States Government or any agency thereof. 


\section{DISCLAIMER}

Portions of this document may be illegible in electronic image products. Images are produced from the best available original document. 
NUREG/CR-1133 Appendix

ANL/ES-81 Appendix

Distribution Code: $\mathrm{RH}$

\title{
ARGONNE NATIONAL LABORATORY
}

9700 South Cass Avenue

Argonne, Illinois 60439

APPENDIX TO

\section{RADON AND RADON-DAUGHTER CONCENTRATIONS IN AIR}

IN THE VICINITY OF THE ANACONDA URANIUM MILL

by

Michae1 H. Momeni

J.B. Lindstrom

C.E. Dungey

Walter E. Kisieleski

Division of Environmental Impact Studies

Project Manager

Walter E. Kisieleski

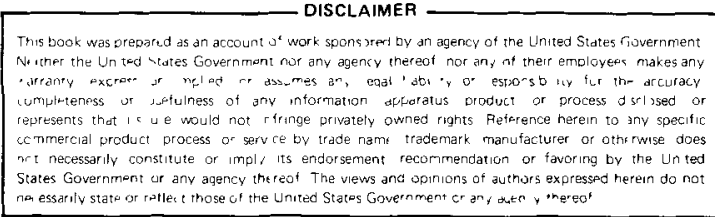

\begin{abstract}
November 1979
Prepared for

Office of Nuclear Regulatory Research

U.S. Nuclear Regulatory Commission

Washington, DC 20555

Under Interagency Agreement DOE 40-550-75

NRC FIN No. A2209
\end{abstract}


$\bullet$

○ 


\section{CONTENTS}

Page

SITE CHARACTERISTICS

Table Al . . . . . . . . . . . . . . . . . . . . .

METEOROLOGY

Tables A2-A9......................... 2

RADON CONCENTRATION IN AIR

Figures A1-A10 . . . . . . . . . . . . . . . . . . . . 6

WORKING LEVEL

Figures A11-A16 . . . . . . . . . . . . . . . . . . . . 
Table Al. Stratigraphic Description and Water-Bearing Characteristics ${ }^{a}$

\begin{tabular}{|c|c|c|c|c|c|}
\hline Age & Unst & Thlckness & $3(f t)$ & Lithologic Characteristics & water-Bearzing Characterzstics \\
\hline \multirow[t]{3}{*}{ Quaternary } & Recent alluvium & $0-$ & 50 & $\begin{array}{l}\text { Valley-fill deposits of unconsolidated silt, } \\
\text { clay, Band, and gravel. }\end{array}$ & $\begin{array}{l}\text { Alluvium and basalt form a single, impor- } \\
\text { cant aquifer. Basal alluvium most produc- } \\
\text { tive portion. Weter quality usually good. }\end{array}$ \\
\hline & Basalt & $0-$ & 200 & $\begin{array}{l}\text { Dense to vesicular basalt, extruded as lava } \\
\text { flows of varying thickness and extent. }\end{array}$ & \\
\hline & Older alluvium & $0-$ & 100 & $\begin{array}{l}\text { Valley-fill deposits of sand, gravel, silt, } \\
\text { and clay. }\end{array}$ & \\
\hline $\begin{array}{l}\text { Quaternary (") } \\
\text { and Tertiary }\end{array}$ & Basaltic rocks & - & & Basaltic cinder cones, plugs, and dikes. & Unknuwa. \\
\hline Iertiary & Extrusive rocks & $0-$ & 300 & Basalt, rhyolite, and tuff breccia. & $\begin{array}{l}\text { Yields water to springs at base of basalt } \\
\text { at favorable locations. }\end{array}$ \\
\hline \multirow[t]{3}{*}{ Cretactous } & $\begin{array}{l}\text { Mesaverde Group, } \\
\text { undivided }\end{array}$ & $1000-1$ & 1500 & $\begin{array}{l}\text { Silty shale and thin- to thick-bedded fine- } \\
\text { gratned sandstone, with some local coal } \\
\text { beda. }\end{array}$ & Not utilized in mine or mill vicinity. \\
\hline & Mancos Shale & $700-$ & 800 & $\begin{array}{l}\text { Platy, calcareous marine shale, with sotne } \\
\text { thick-bedded sandstones in lower part. }\end{array}$ & $\begin{array}{l}\text { Ires Hermanos sandstone member yields } \\
\text { small amounts of falr-quality water in } \\
\text { mine vicinity. }\end{array}$ \\
\hline & Dakota Sandstone & $50-$ & 100 & $\begin{array}{l}\text { Massive, medium- to coarse-gralned sand- } \\
\text { stone, locally with interbedded siltstone. }\end{array}$ & $\begin{array}{l}\text { Not utilized for water supply in mine or } \\
\text { mill area. }\end{array}$ \\
\hline \multirow[t]{2}{*}{ Jurassic } & Morrison Formation & $300-$ & 500 & $\begin{array}{l}\text { Brushy Basin (upper) member consists of } \\
\text { mudstone and arkosic sandstone, contains } \\
\text { Jackpile sandstone at top of unit, Waste- } \\
\text { water Canyon member, sandstone and } \\
\text { mudstone. Recapture Creek (lower) menber, } \\
\text { alternating sandstone and afltstone or } \\
\text { mudstone. }\end{array}$ & $\begin{array}{l}\text { Brushy Basin and Westwater Canyon members } \\
\text { yleld amall quantities of falr- to poor- } \\
\text { quality water. Recapture creek member not } \\
\text { known to yield groundwater. }\end{array}$ \\
\hline & $\begin{array}{l}\text { San Rafael Group, } \\
\text { und z vided }\end{array}$ & $450-$ & 700 & $\begin{array}{l}\text { Fine- to medium-grained, in part gilty } \\
\text { sandstone, siltstone, and claystone, with } \\
\text { some limestone in lower part, and massive } \\
\text { croas-bedded gandstone in basal part. }\end{array}$ & $\begin{array}{l}\text { Bluff sandstone formation is utilized for } \\
\text { stock watering in Jackpile mine area. } \\
\text { Yields small quantitles of poor-quality } \\
\text { water. }\end{array}$ \\
\hline \multirow[t]{2}{*}{ Trzassic } & Wingate Sandetone & $60-$ & 100 & Massive, crossmbedded aandstone. & Not utilized in mine or mill areas. \\
\hline & Chinle Formation & $1400-1$ & 1600 & $\begin{array}{l}\text { Siltstone and mudstone, with interbedded } \\
\text { silty sandstone and some conglomeratic } \\
\text { sandstone. Some thin limestones in the } \\
\text { upper part, a thick sandstone unit near } \\
\text { the aiddle, considerable sandstone in the } \\
\text { lower part. }\end{array}$ & $\begin{array}{l}\text { Utilized for domestic-stock watering and } \\
\text { occaslonally Irrigation supply. Water } \\
\text { quality falr to poor. }\end{array}$ \\
\hline \multirow[t]{4}{*}{ Permian } & San Andres Limestone & $80-$ & 150 & $\begin{array}{l}\text { Thick-bedded to massive limestone, sandy } \\
\text { limestone, and limy sandstone. The } \\
\text { limestone strat are cavernous in many } \\
\text { localities. }\end{array}$ & \multirow[t]{2}{*}{$\begin{array}{l}\text { San Andree and Gloriete formations consti- } \\
\text { tute a single aquifer. Most highly } \\
\text { developed aquifer in Bluewater area. } \\
\text { Water quality good near outcrop; deteri- } \\
\text { orates with distance from outcrop. }\end{array}$} \\
\hline & Glorieta Sandatone & $125-$ & 300 & $\begin{array}{l}\text { Thick-bedded to assive, well-sorted } \\
\text { medium-grained eandstone. Some inter- } \\
\text { bedded glltstone in besal part. }\end{array}$ & \\
\hline & Yeso Formation & $350-$ & 500 & $\begin{array}{l}\text { Upper part fine-grained silty sandstone } \\
\text { and mudstone with some layers of evapo- } \\
\text { rites and limestone. Lower part fine- } \\
\text { grained sandstone. }\end{array}$ & $\begin{array}{l}\text { Yields to several wells on flank of Zuni } \\
\text { uplift. Except in outcrop areas, water } \\
\text { ls of poor quality. }\end{array}$ \\
\hline & Abo Formation & $500-$ & 800 & $\begin{array}{l}\text { Arkosic sandstone and s } 1 \text { listone, with } \\
\text { numerous layers of conglomerate in lower } \\
\text { part. }\end{array}$ & $\begin{array}{l}\text { Not utilized in area. Contains water of } \\
\text { poor quality and is deeply buried. }\end{array}$ \\
\hline Penneylvanian (?) & & $0-$ & 480 & $\begin{array}{l}\text { Arkose and conglomerate, and a few thin } \\
\text { arkosic-limestone lenses and beds of shale. }\end{array}$ & Unknown. \\
\hline Pre-Cambrian & & & & $\begin{array}{l}\text { Granite, gneiss, metarhyolite, schist, and } \\
\text { greenstone. }\end{array}$ & Unknown. \\
\hline
\end{tabular}

a From Anaconda (1976). 
Table A2. Annual Frequency Distribution, Stability Class A

\begin{tabular}{rrrrrrrrr}
\hline & \multicolumn{7}{c}{ Windspeed $(\mathrm{mph})$} & \\
\cline { 2 - 7 } Direction & $0-3$ & $4-6$ & $7-11$ & $12-16$ & $17-25$ & $>25$ & Total \\
\hline N & 24 & 9 & 1 & 0 & 0 & 0 & 34 \\
NNE & 11 & 5 & 1 & 0 & 0 & 0 & 17 \\
NE & 8 & 8 & 2 & 0 & 0 & 0 & 18 \\
ENE & 12 & 5 & 1 & 0 & 0 & 0 & 18 \\
E & 7 & 2 & 1 & 0 & 0 & 0 & 10 \\
ESE & 5 & 2 & 1 & 0 & 0 & 0 & 8 \\
SE & 0 & 0 & 0 & 0 & 0 & 0 & 0 \\
SSE & 0 & 0 & 0 & 0 & 0 & 0 & 0 \\
S & 1 & 0 & 0 & 0 & 0 & 0 & 1 \\
SSW & 3 & 1 & 0 & 0 & 0 & 0 & 4 \\
SW & 7 & 0 & 0 & 0 & 0 & 0 & 7 \\
WSW & 7 & 3 & 1 & 0 & 0 & 0 & 11 \\
W & 10 & 2 & 1 & 0 & 0 & 0 & 13 \\
WNW & 12 & 7 & 2 & 0 & 0 & 0 & 21 \\
NW & 20 & 7 & 0 & 0 & 0 & 0 & 27 \\
NNW & 19 & 7 & 0 & 0 & 0 & 0 & 26 \\
\hline
\end{tabular}

Table A3. Annua1 Frequency Distribution, Stability Class B

\begin{tabular}{rrrrrrrrr}
\hline & \multicolumn{7}{c}{ Windspeed $(\mathrm{mph})$} & \\
\cline { 2 - 6 } Direction & $0-3$ & $4-6$ & $7-11$ & $12-16$ & $17-25$ & $>25$ & Total \\
\hline N & 25 & 8 & 1 & 0 & 0 & 0 & 34 \\
NNE & 17 & 7 & 2 & 0 & 0 & 0 & 26 \\
NE & 17 & 3 & 0 & 0 & 0 & 0 & 20 \\
ENE & 16 & 3 & 0 & 0 & 0 & 0 & 19 \\
E & 14 & 4 & 1 & 0 & 0 & 0 & 19 \\
ESE & 7 & 2 & 1 & 0 & 0 & 0 & 10 \\
SE & 4 & 0 & 0 & 0 & 0 & 0 & 4 \\
SSE & 6 & 1 & 1 & 0 & 0 & 0 & 8 \\
S & 9 & 0 & 0 & 0 & 0 & 0 & 9 \\
SSW & 12 & 6 & 2 & 0 & 0 & 0 & 20 \\
SW & 14 & 2 & 1 & 0 & 0 & 0 & 17 \\
WSW & 14 & 5 & 1 & 1 & 0 & 0 & 21 \\
W & 29 & 7 & 2 & 0 & 0 & 0 & 38 \\
WNW & 26 & 8 & 3 & 0 & 0 & 0 & 37 \\
NW & 33 & 8 & 3 & 0 & 1 & 0 & 45 \\
NNW & 27 & 9 & 3 & 2 & 0 & 0 & 41 \\
\hline
\end{tabular}


Table A4. Annual Frequency Distribution, Stability Class C

\begin{tabular}{rrrrrrrrr}
\hline & \multicolumn{7}{c}{ Windspeed $(\mathrm{mph})$} & \\
\cline { 2 - 7 } Direction & $0-3$ & $4-6$ & $7-11$ & $12-16$ & $17-25$ & $>25$ & Total \\
\hline N & 44 & 32 & 8 & 1 & 0 & 0 & 85 \\
NNE & 39 & 10 & 5 & 0 & 0 & 0 & 54 \\
NE & 26 & 6 & 2 & 0 & 0 & 0 & 34 \\
ENE & 17 & 4 & 1 & 0 & 0 & 0 & 22 \\
E & 27 & 6 & 0 & 0 & 0 & 0 & 33 \\
ESE & 21 & 16 & 2 & 0 & 0 & 0 & 39 \\
SE & 10 & 17 & 2 & 0 & 0 & 0 & 29 \\
SSE & 20 & 12 & 1 & 1 & 0 & 0 & 34 \\
S & 20 & 11 & 10 & 4 & 1 & 0 & 46 \\
SSW & 32 & 15 & 4 & 0 & 0 & 0 & 51 \\
SW & 34 & 15 & 9 & 0 & 0 & 0 & 58 \\
WSW & 62 & 17 & 12 & 0 & 0 & 0 & 91 \\
W & 60 & 17 & 9 & 0 & 0 & 0 & 86 \\
WNW & 52 & 24 & 8 & 1 & 0 & 0 & 85 \\
NW & 49 & 26 & 8 & 1 & 0 & 0 & 84 \\
NNW & 35 & 21 & 5 & 0 & 1 & 0 & 62 \\
\hline
\end{tabular}

Table A5. Annual Frequency Distribution, Stability Class D

\begin{tabular}{rrrrrrrrr}
\hline & \multicolumn{7}{c}{ Windspeed $(\mathrm{mph})$} & \\
\cline { 2 - 7 } Direction & $0-3$ & $4-6$ & $7-11$ & $12-16$ & $17-25$ & $>25$ & Total \\
\hline N & 72 & 40 & 40 & 1 & 0 & 0 & 153 \\
NNE & 62 & 19 & 10 & 2 & 0 & 0 & 93 \\
NE & 40 & 8 & 5 & 0 & 0 & 0 & 53 \\
ENE & 49 & 16 & 3 & 1 & 0 & 0 & 69 \\
E & 46 & 22 & 1 & 0 & 0 & 1 & 70 \\
ESE & 67 & 33 & 22 & 0 & 0 & 0 & 122 \\
SE & 46 & 58 & 46 & 2 & 0 & 0 & 152 \\
SSE & 51 & 43 & 36 & 6 & 1 & 0 & 137 \\
S & 37 & 33 & 15 & 5 & 3 & 0 & 93 \\
SSW & 83 & 44 & 25 & 7 & 1 & 0 & 160 \\
SW & 91 & 38 & 44 & 20 & 8 & 0 & 201 \\
WSW & 99 & 53 & 64 & 40 & 8 & 0 & 264 \\
W & 164 & 47 & 68 & 24 & 2 & 0 & 305 \\
WNW & 151 & 54 & 63 & 22 & 1 & 0 & 291 \\
NW & 114 & 56 & 53 & 5 & 2 & 0 & 230 \\
NNW & 110 & 59 & 54 & 5 & 0 & 0 & 228 \\
\hline
\end{tabular}


Table A6. Annual Frequency Distribution, Stability Class E

\begin{tabular}{rrrrrrrrr}
\hline & \multicolumn{7}{c}{ Windspeed $(\mathrm{mph})$} & \\
\cline { 2 - 7 } Direction & $0-3$ & $4-6$ & $7-11$ & $12-16$ & $17-25$ & $>25$ & Total \\
\hline N & 47 & 30 & 28 & 4 & 1 & 0 & 110 \\
NNE & 21 & 11 & 10 & 2 & 0 & 0 & 44 \\
NE & 18 & 5 & 5 & 1 & 0 & 1 & 30 \\
ENE & 14 & 6 & 2 & 2 & 0 & 0 & 24 \\
E & 18 & 7 & 4 & 1 & 0 & 0 & 30 \\
ESE & 11 & 21 & 13 & 0 & 0 & 0 & 45 \\
SE & 17 & 33 & 27 & 1 & 0 & 0 & 78 \\
SSE & 35 & 59 & 89 & 17 & 7 & 1 & 208 \\
S & 57 & 28 & 52 & 16 & 8 & 6 & 167 \\
SSW & 79 & 33 & 32 & 9 & 15 & 4 & 172 \\
SW & 76 & 78 & 39 & 25 & 17 & 0 & 235 \\
WSW & 96 & 48 & 79 & 77 & 46 & 6 & 352 \\
W & 178 & 65 & 89 & 66 & 24 & 0 & 422 \\
WNW & 170 & 100 & 74 & 31 & 25 & 1 & 401 \\
NW & 98 & 101 & 77 & 46 & 20 & 0 & 342 \\
NNW & 69 & 114 & 67 & 6 & 4 & 0 & 260 \\
\hline
\end{tabular}

Table A7. Annual Frequency Distribution, Stability Class F

\begin{tabular}{rrrrrrrrr}
\hline & \multicolumn{7}{c}{ Windspeed $(\mathrm{mph})$} & \\
\cline { 2 - 6 } Direction & $0-3$ & $4-6$ & $7-11$ & $12-16$ & $17-25$ & $>25$ & Total \\
\hline N & 2 & 4 & 4 & 0 & 0 & 0 & 10 \\
NNE & 1 & 2 & 0 & 0 & 0 & 0 & 3 \\
NE & 1 & 1 & 0 & 1 & 0 & 0 & 3 \\
ENE & 0 & 1 & 0 & 0 & 0 & 0 & 1 \\
E & 0 & 0 & 1 & 0 & 0 & 0 & 1 \\
ESE & 0 & 1 & 0 & 0 & 0 & 0 & 1 \\
SE & 0 & 2 & 3 & 0 & 0 & 0 & 5 \\
SSE & 1 & 2 & 16 & 1 & 0 & 0 & 20 \\
S & 8 & 12 & 24 & 4 & 2 & 1 & 51 \\
SSW & 16 & 8 & 8 & 6 & 0 & 1 & 39 \\
SW & 16 & 12 & 17 & 5 & 2 & 1 & 53 \\
WSW & 7 & 9 & 21 & 8 & 4 & 7 & 56 \\
W & 21 & 5 & 4 & 3 & 1 & 1 & 35 \\
WNW & 33 & 10 & 14 & 9 & 6 & 0 & 72 \\
NW & 12 & 11 & 25 & 9 & 5 & 0 & 62 \\
NNW & 3 & 19 & 23 & 0 & 0 & 0 & 45 \\
\hline
\end{tabular}


Table A8. Annual Frequency Distribution, Stability Class G

\begin{tabular}{|c|c|c|c|c|c|c|c|}
\hline \multirow[b]{2}{*}{ Direction } & \multicolumn{6}{|c|}{ Windspeed (mph) } & \multirow[b]{2}{*}{ Total } \\
\hline & $0-3$ & $4-6$ & $7-11$ & $12-16$ & $17-25$ & $>25$ & \\
\hline $\mathrm{N}$ & 0 & 1 & 0 & 0 & 0 & 0 & 1 \\
\hline NNE & 0 & 0 & 0 & 0 & 0 & 0 & 0 \\
\hline $\mathrm{NE}$ & 1 & 0 & 0 & 0 & 0 & 0 & 1 \\
\hline ENE & 1 & 0 & 0 & 0 & 0 & 0 & 1 \\
\hline $\mathrm{E}$ & 3 & 0 & 0 & 0 & 0 & 0 & 3 \\
\hline ESE & 0 & 0 & 0 & 0 & 0 & 0 & 0 \\
\hline$S E$ & 0 & 0 & 0 & 0 & 0 & 0 & 0 \\
\hline SSE & 0 & 0 & 0 & 1 & 0 & 0 & 1 \\
\hline $\mathrm{s}$ & 0 & 1 & 5 & 0 & 0 & 0 & 6 \\
\hline SSW & 0 & 1 & 1 & 1 & 2 & 0 & 5 \\
\hline SW & 5 & 5 & 3 & 0 & 0 & 0 & 13 \\
\hline WSW & 12 & 3 & 4 & 1 & 0 & 0 & 20 \\
\hline W & 1 & $?$ & 2 & 0 & 0 & 0 & 5 \\
\hline WNW & 1 & 0 & 1 & 1 & 1 & 0 & 4 \\
\hline NW & 1 & 1 & 3 & 0 & 0 & 0 & 5 \\
\hline NNW & 0 & 1 & 0 & 0 & 0 & 0 & 1 \\
\hline
\end{tabular}

Table A9. Annual Frequency Distribution, A11 Stability Classes

\begin{tabular}{|c|c|c|c|c|c|c|c|}
\hline \multirow[b]{2}{*}{ Direction } & \multicolumn{6}{|c|}{ Windspeed (mph) } & \multirow[b]{2}{*}{ Tota } \\
\hline & $0-3$ & $4-6$ & $7-11$ & $12-16$ & $17-25$ & $>25$ & \\
\hline $\mathrm{N}$ & 214 & 124 & 82 & 6 & 1 & 0 & 427 \\
\hline NNE & 151 & 54 & 28 & 4 & 0 & 0 & 237 \\
\hline $\mathrm{NE}$ & 111 & 31 & 14 & 2 & 0 & 1 & 159 \\
\hline ENE & 109 & 35 & 7 & 3 & 0 & 0 & 154 \\
\hline E & 115 & 41 & 8 & 1 & 0 & 1 & 166 \\
\hline ESE & 111 & 75 & 39 & 0 & 0 & 0 & 225 \\
\hline$S E$ & 77 & 110 & 78 & 3 & 0 & 0 & 268 \\
\hline SSE & 113 & 117 & 143 & 26 & 8 & 1 & 408 \\
\hline$S$ & 132 & 85 & 106 & 29 & 14 & 7 & 373 \\
\hline SSW & 225 & 108 & 72 & 23 & 18 & 5 & 451 \\
\hline SW & 243 & 150 & 113 & 50 & 27 & 1 & 584 \\
\hline WSW & 297 & 138 & 182 & 127 & 58 & 13 & 815 \\
\hline W & 463 & 145 & 175 & 93 & 27 & 1 & 904 \\
\hline WNW & 445 & 203 & 165 & 64 & 33 & 1 & 911 \\
\hline NW & 327 & 210 & 169 & 61 & 28 & 0 & 795 \\
\hline NNW & 263 & 230 & 152 & 13 & 5 & 0 & 663 \\
\hline
\end{tabular}


STATION 701, DECEMBER 1977

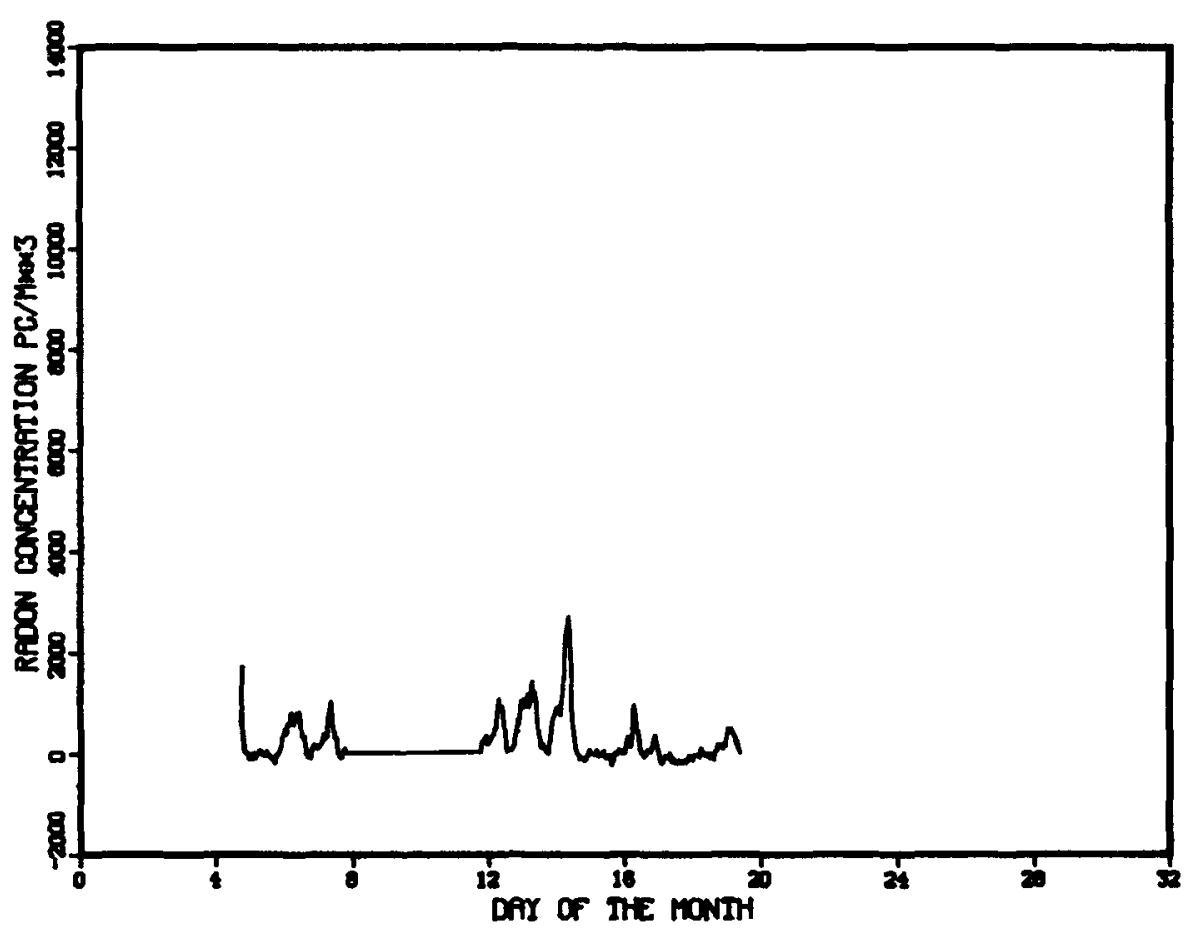

STATION 701, JANUARY 1978

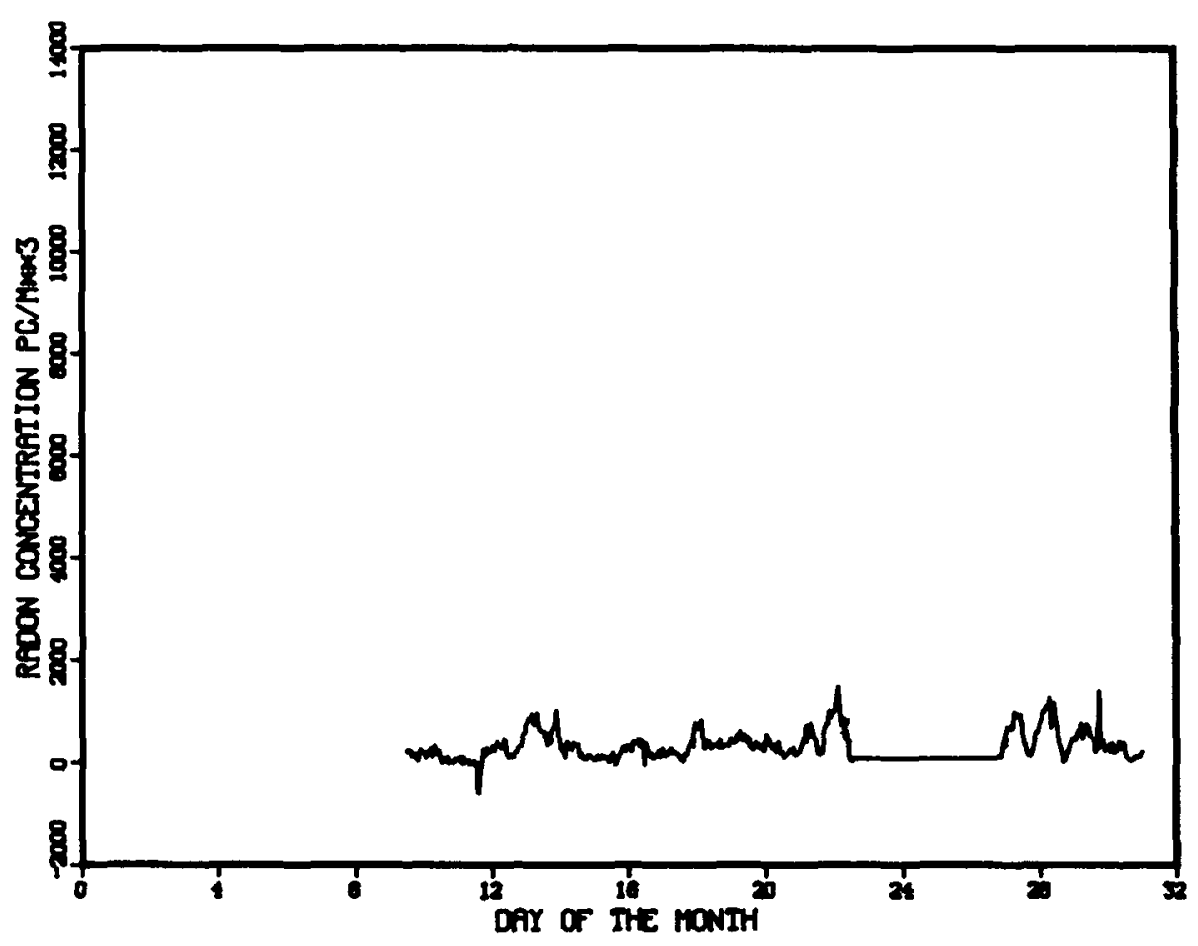

Figure Al. Radon Concentration by Hour at the Elkins Background Station 701 . 
STATION 701, APRIL 1978

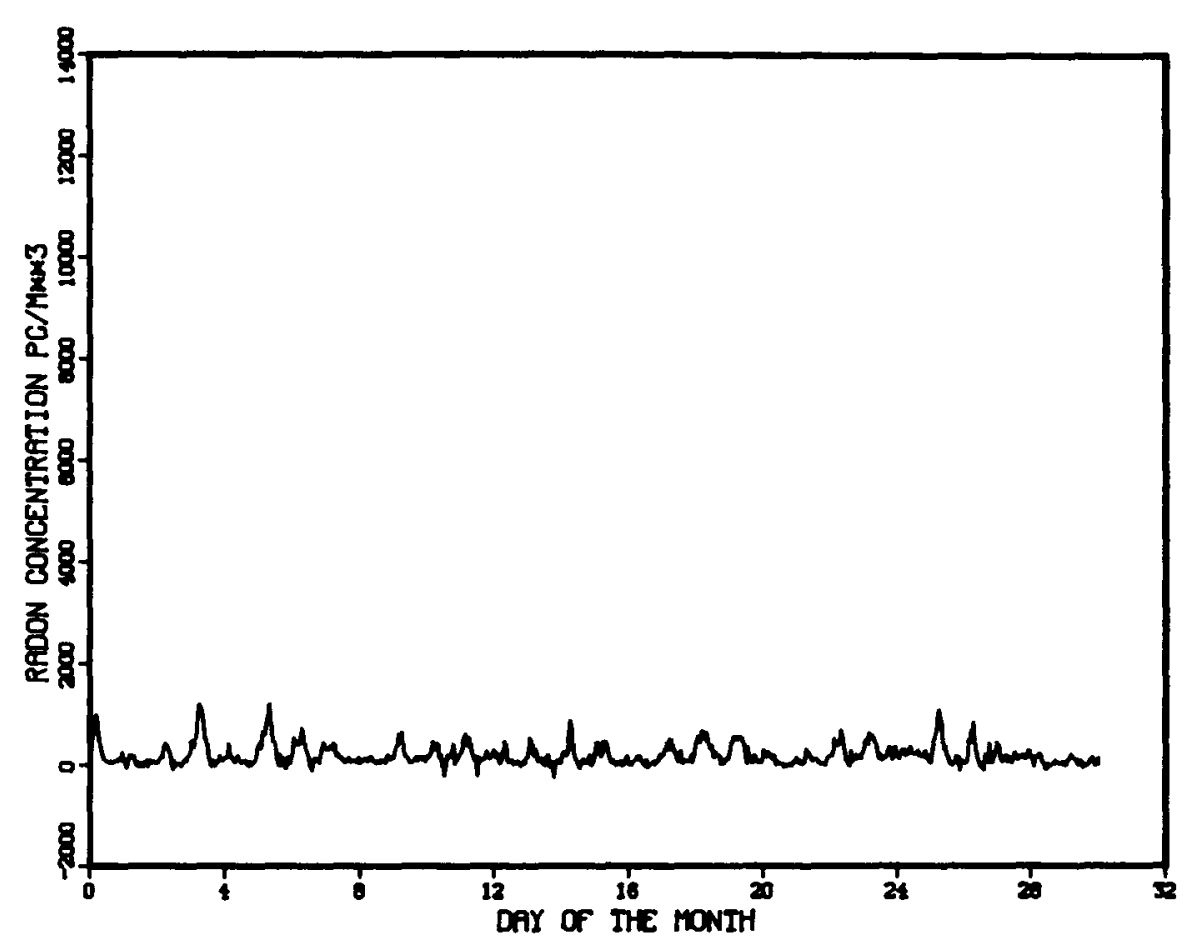

STATION 701, MAY 1978

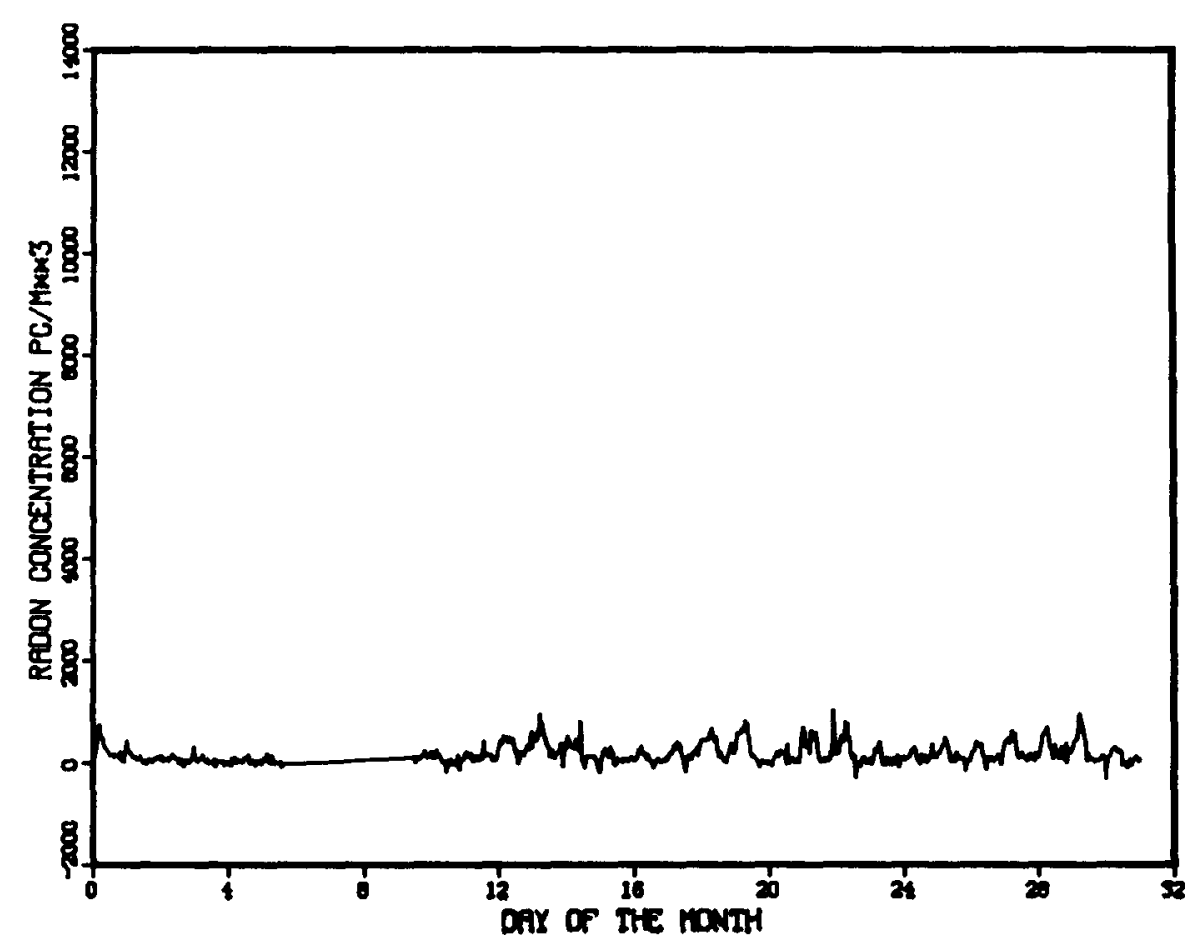

Figure Al. Continued. 
STATION 701, JUNE 1978

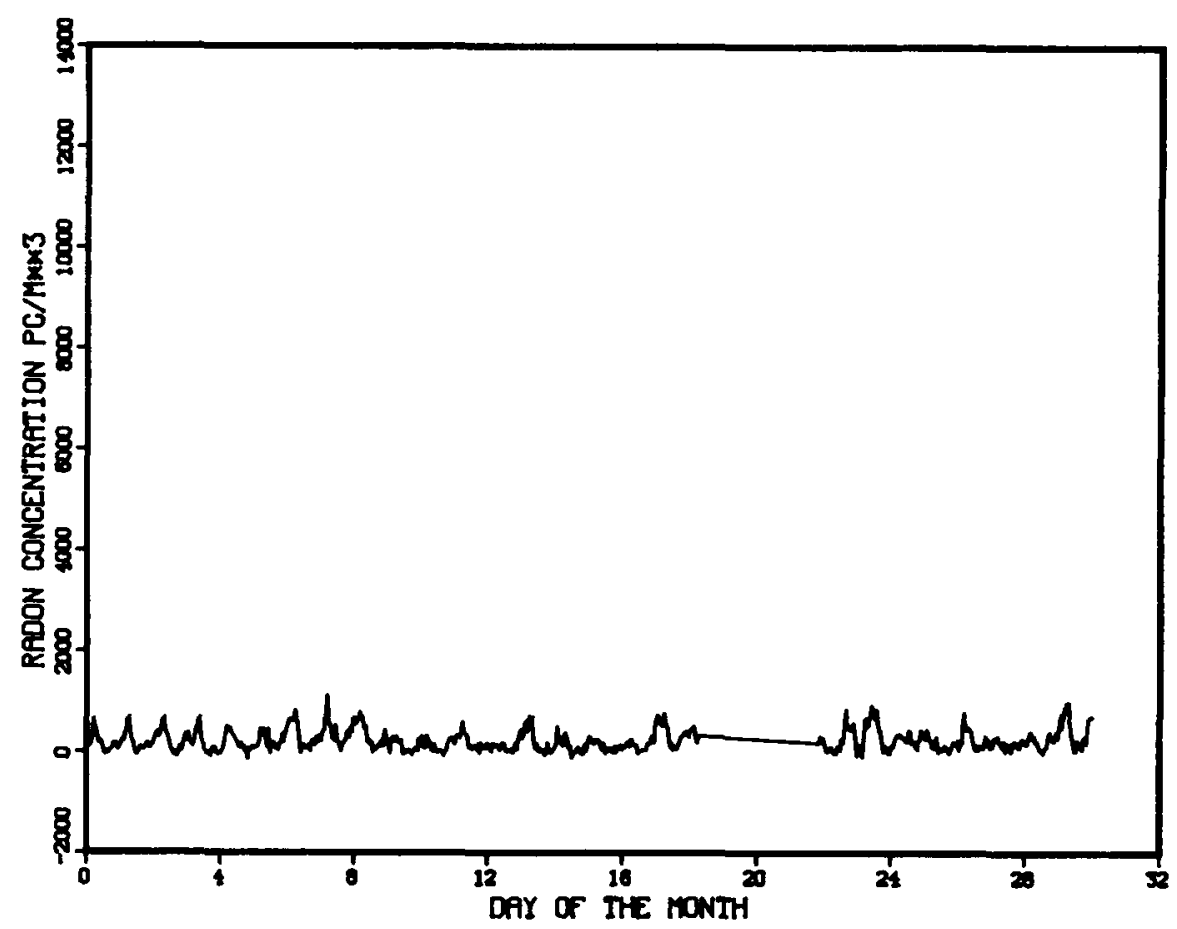

STATION 701, JULY 1978

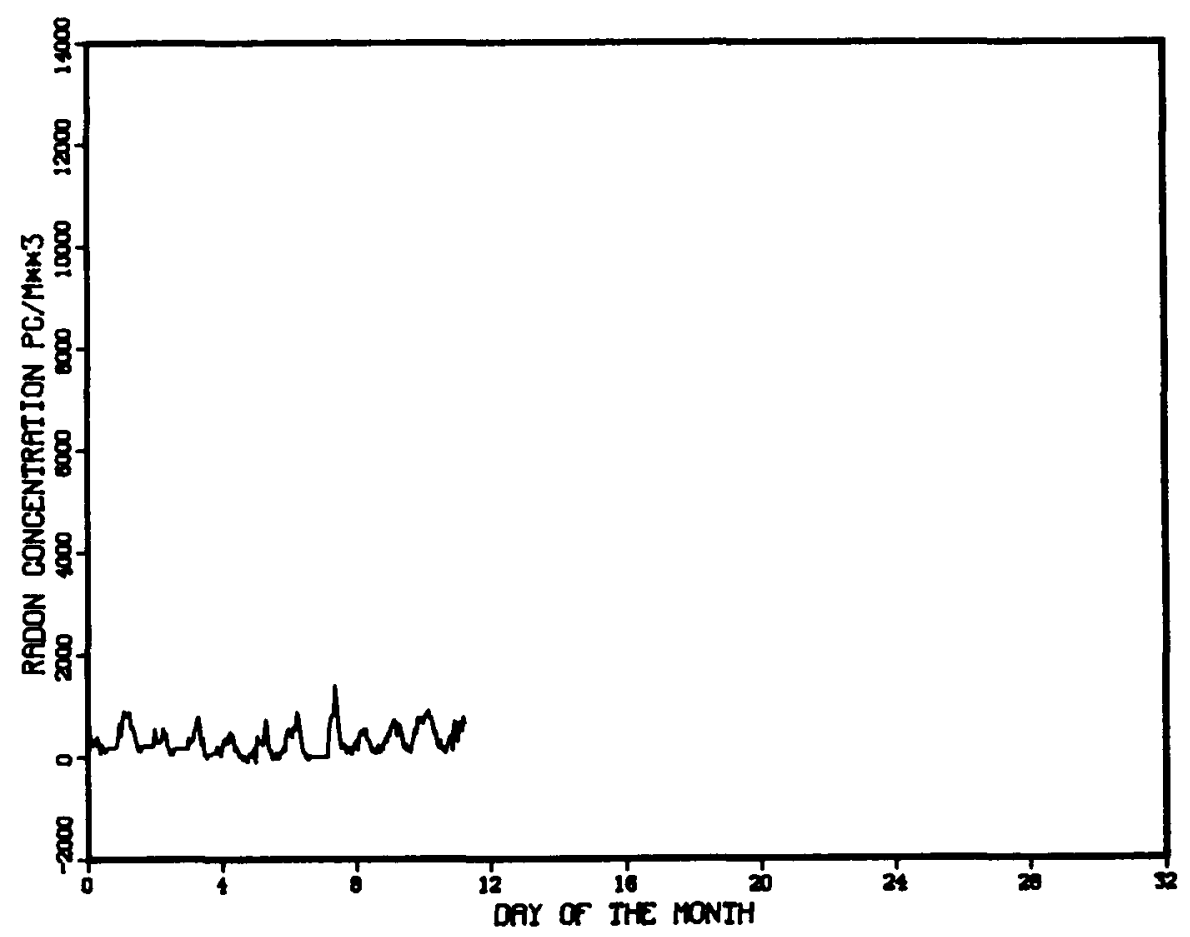

Figure Al. Continued. 
STATION 701, SEPTEMBER 1978

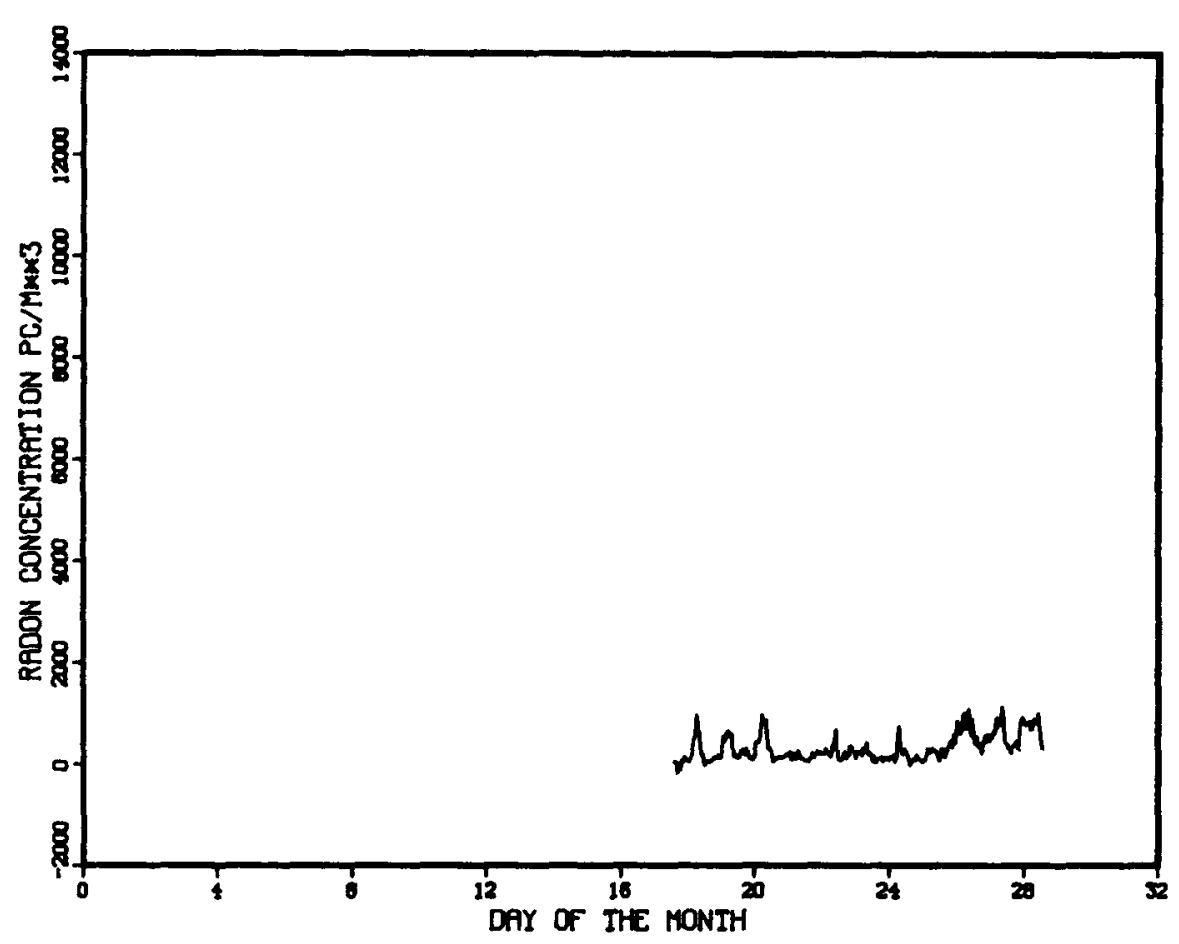

STATION 701, OCTOBER 1978

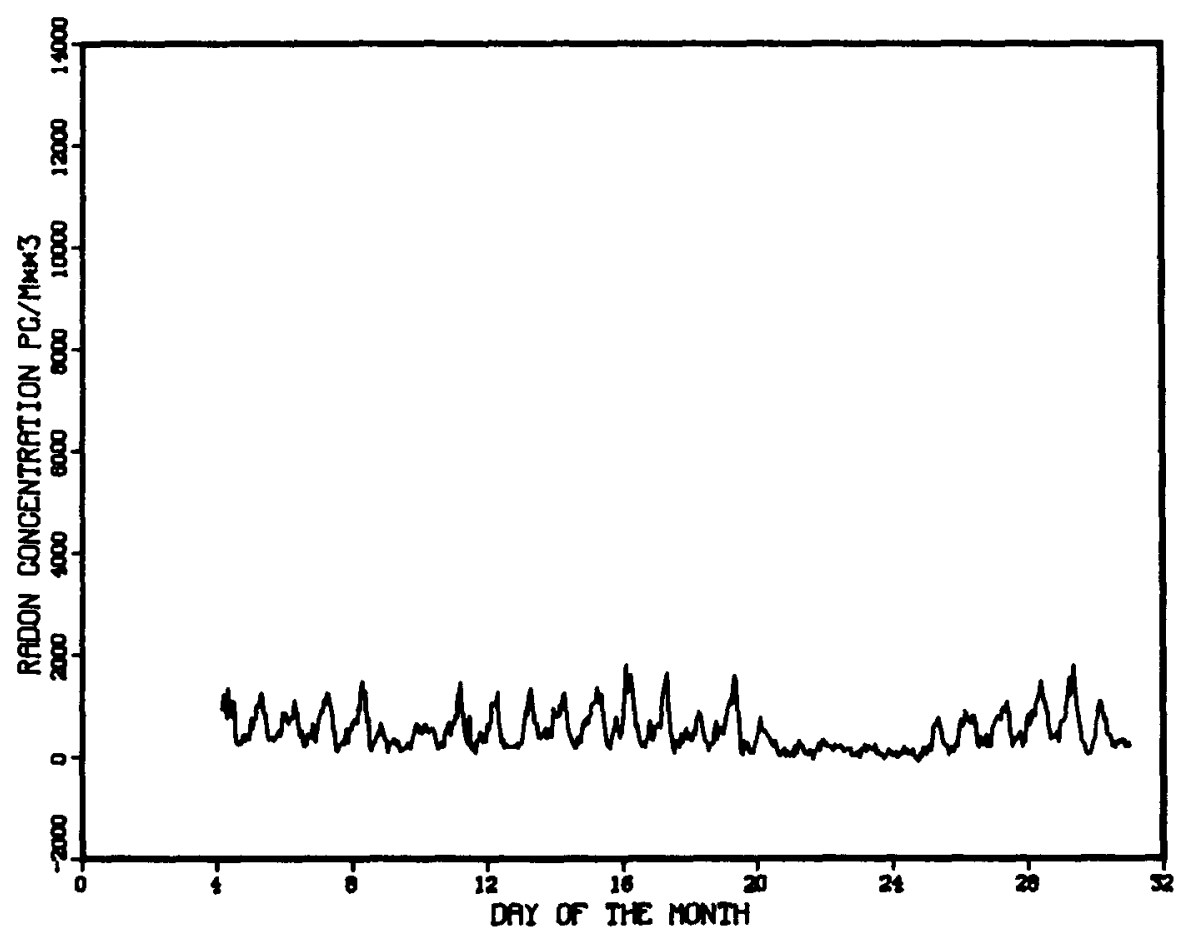

Figure Al. Continued. . 
STATION 701, NOVEMBER 1978

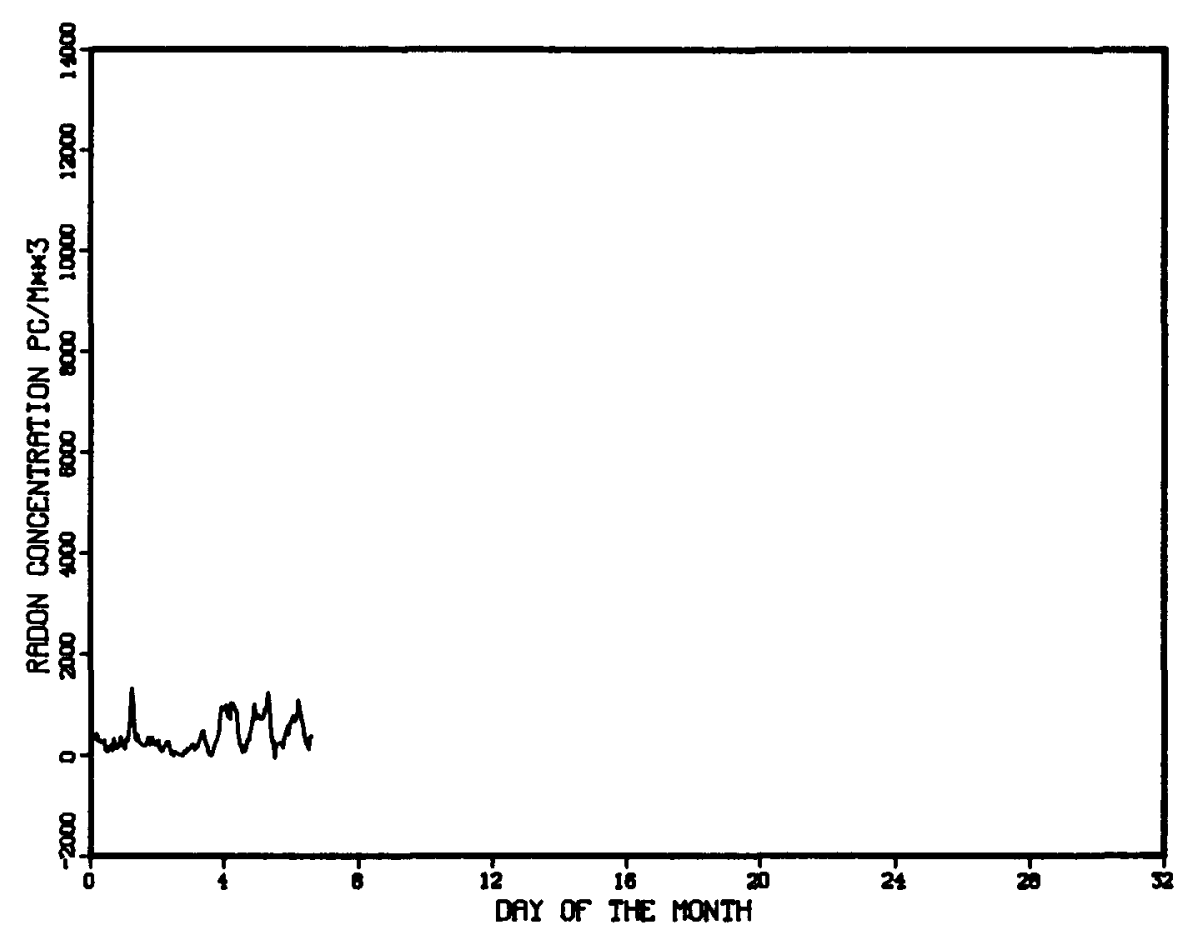

Figure Al. Continued. 
STATION 701, DECEMBER 1977

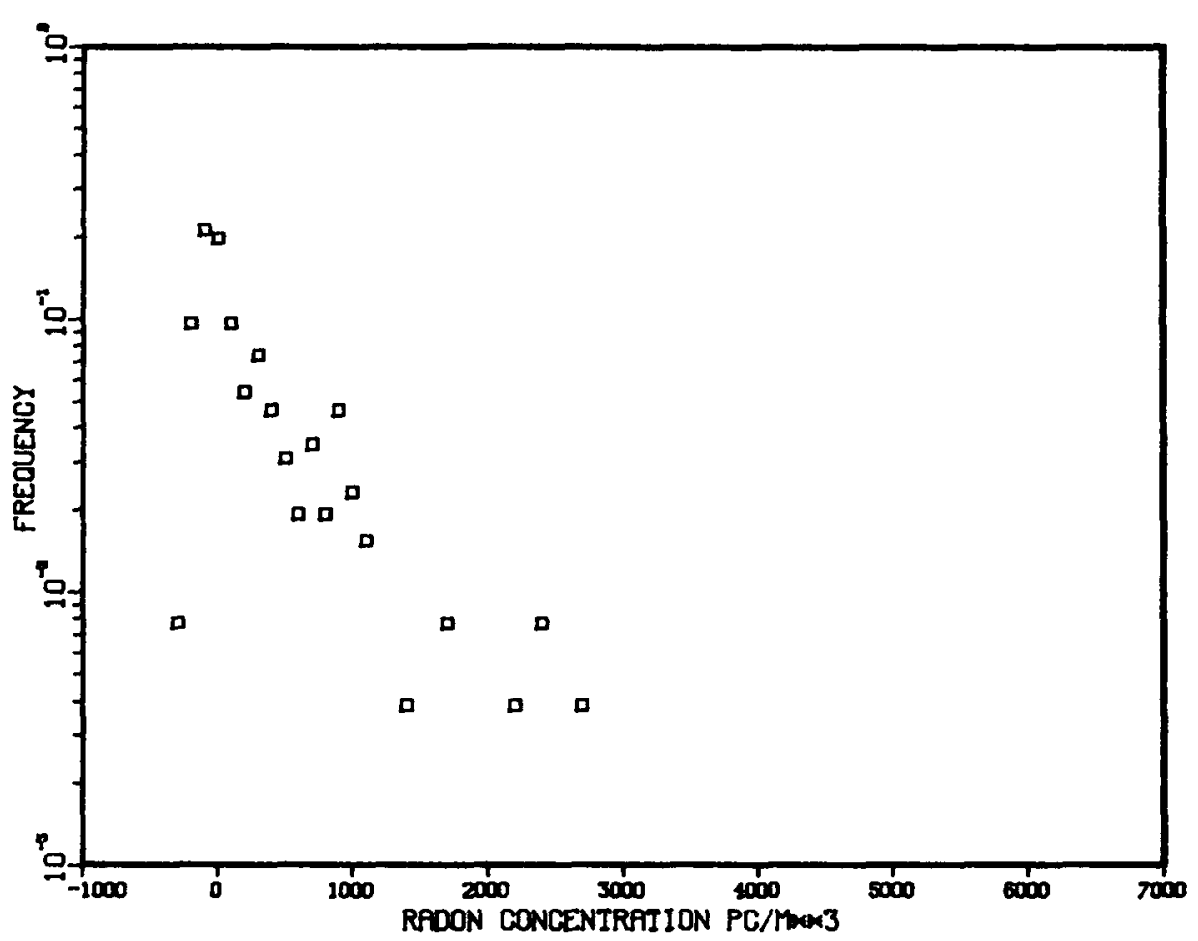

STATION 701, JANUARY 1978

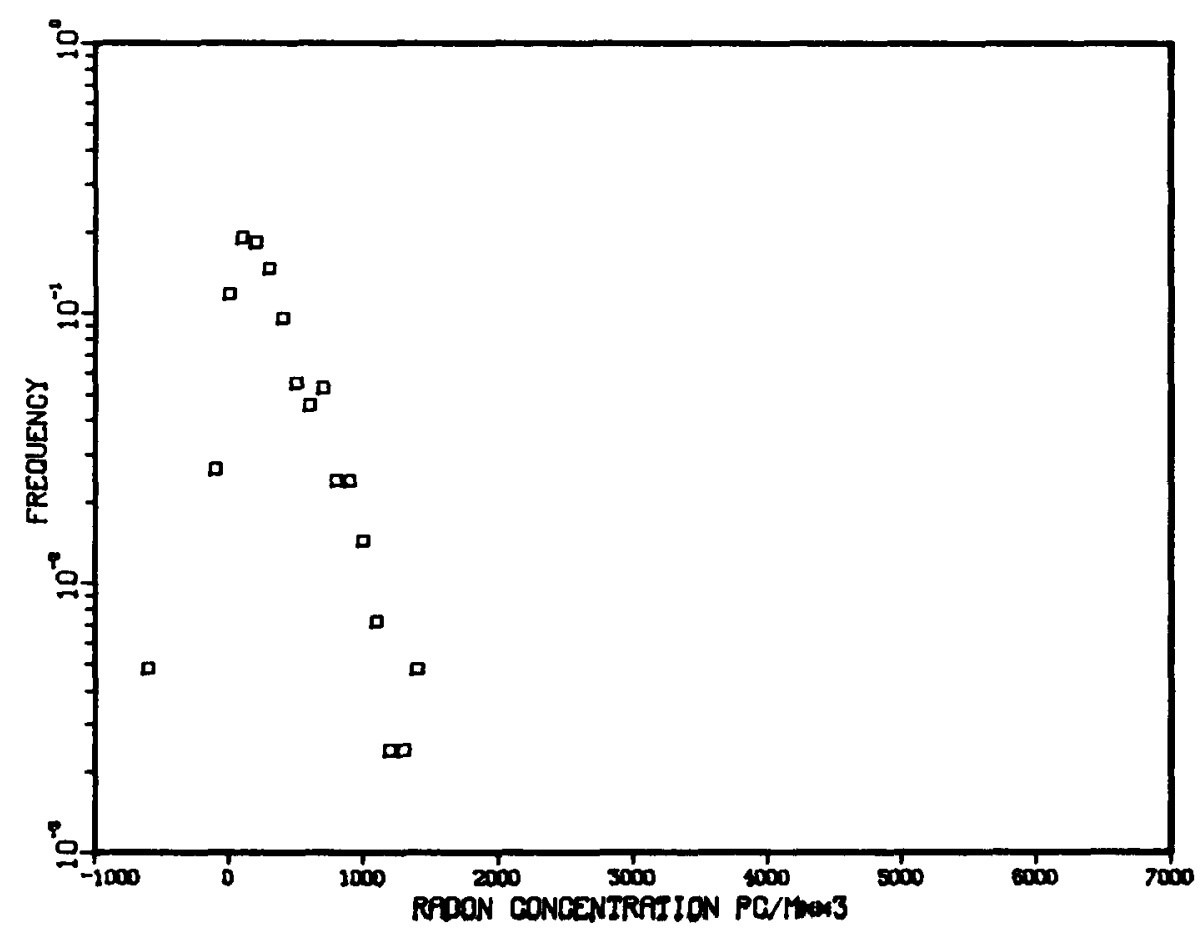

Figure A2. Radon-Concentration Frequency at the Elkins Background Station 701 . 
STATION 701, APRIL 1978

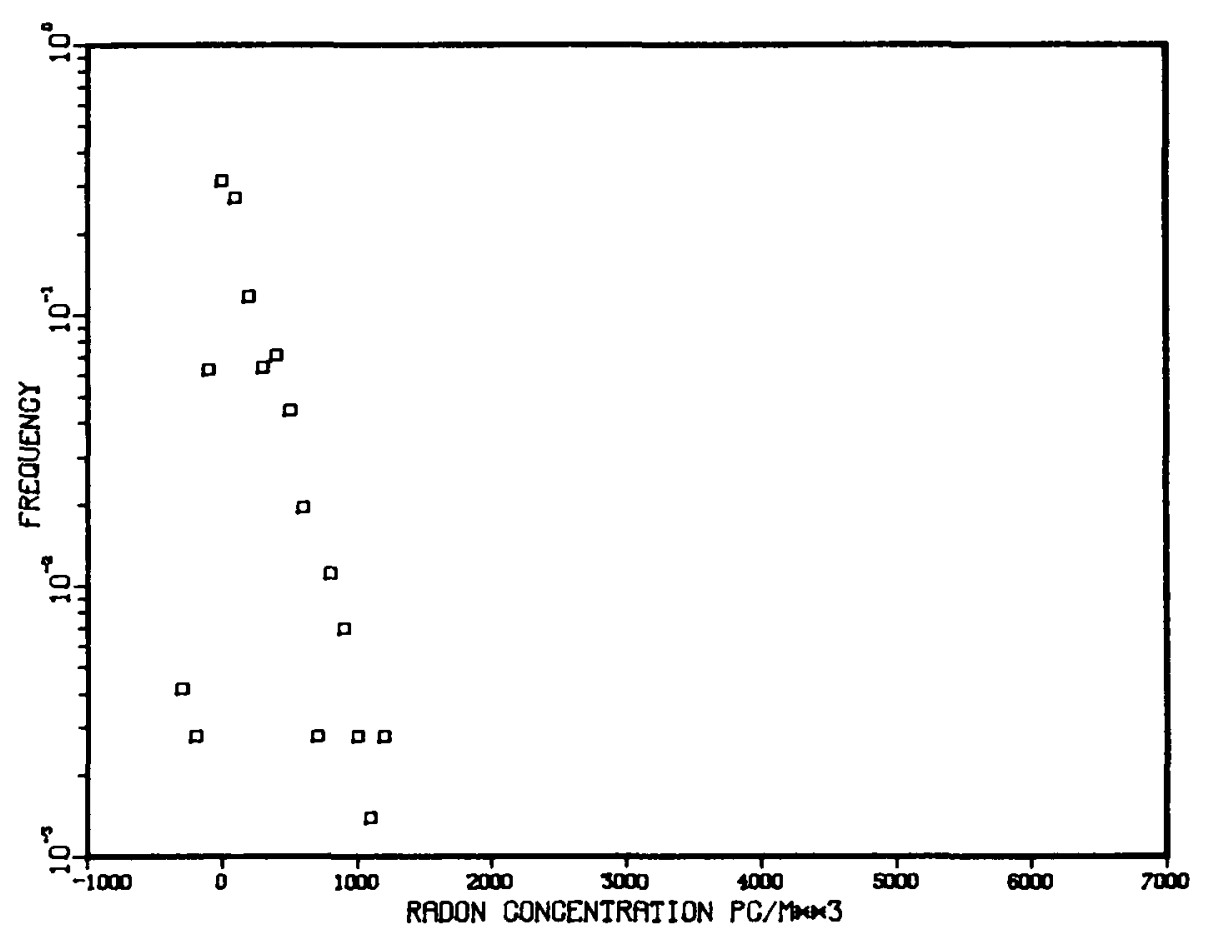

STATION 701, MAY 1978

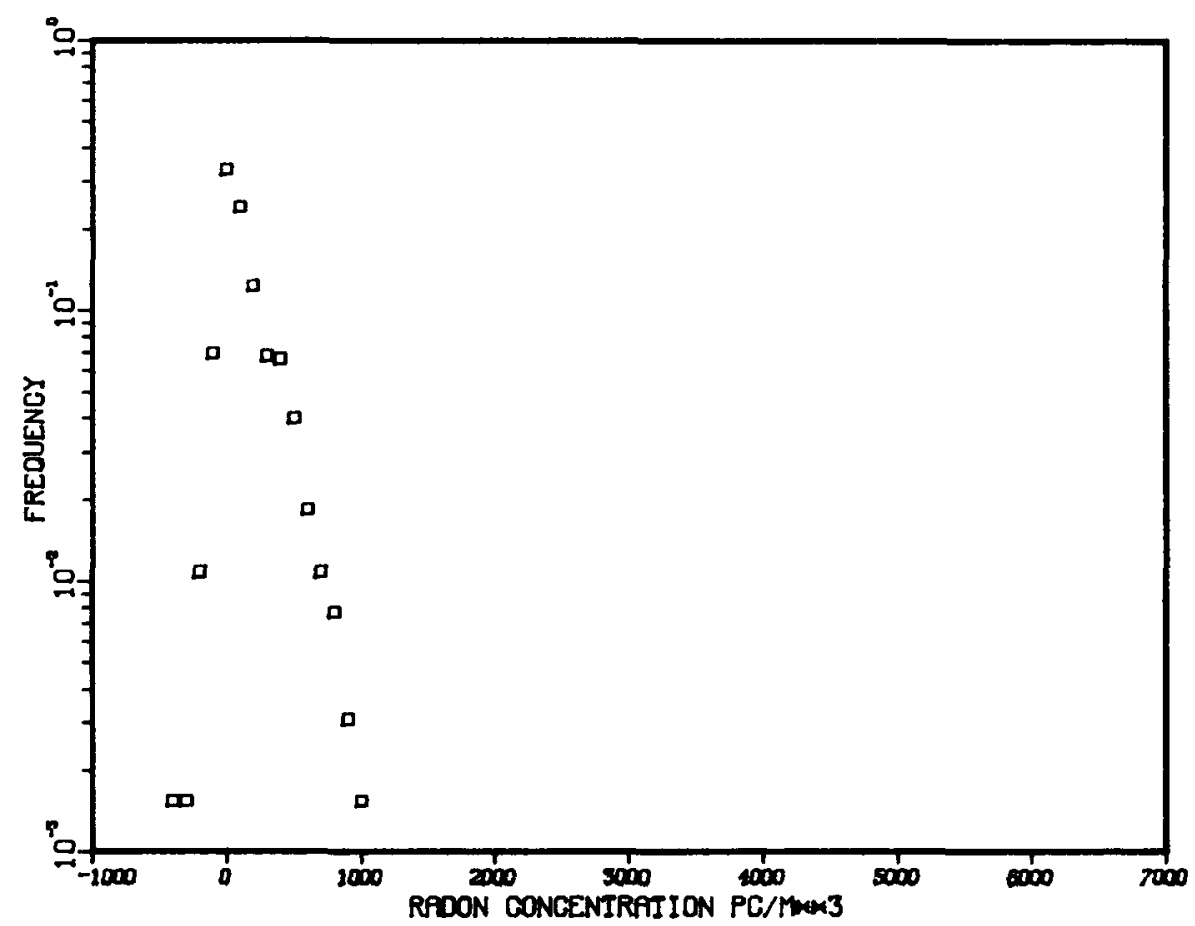

Figure A2. Continued. 


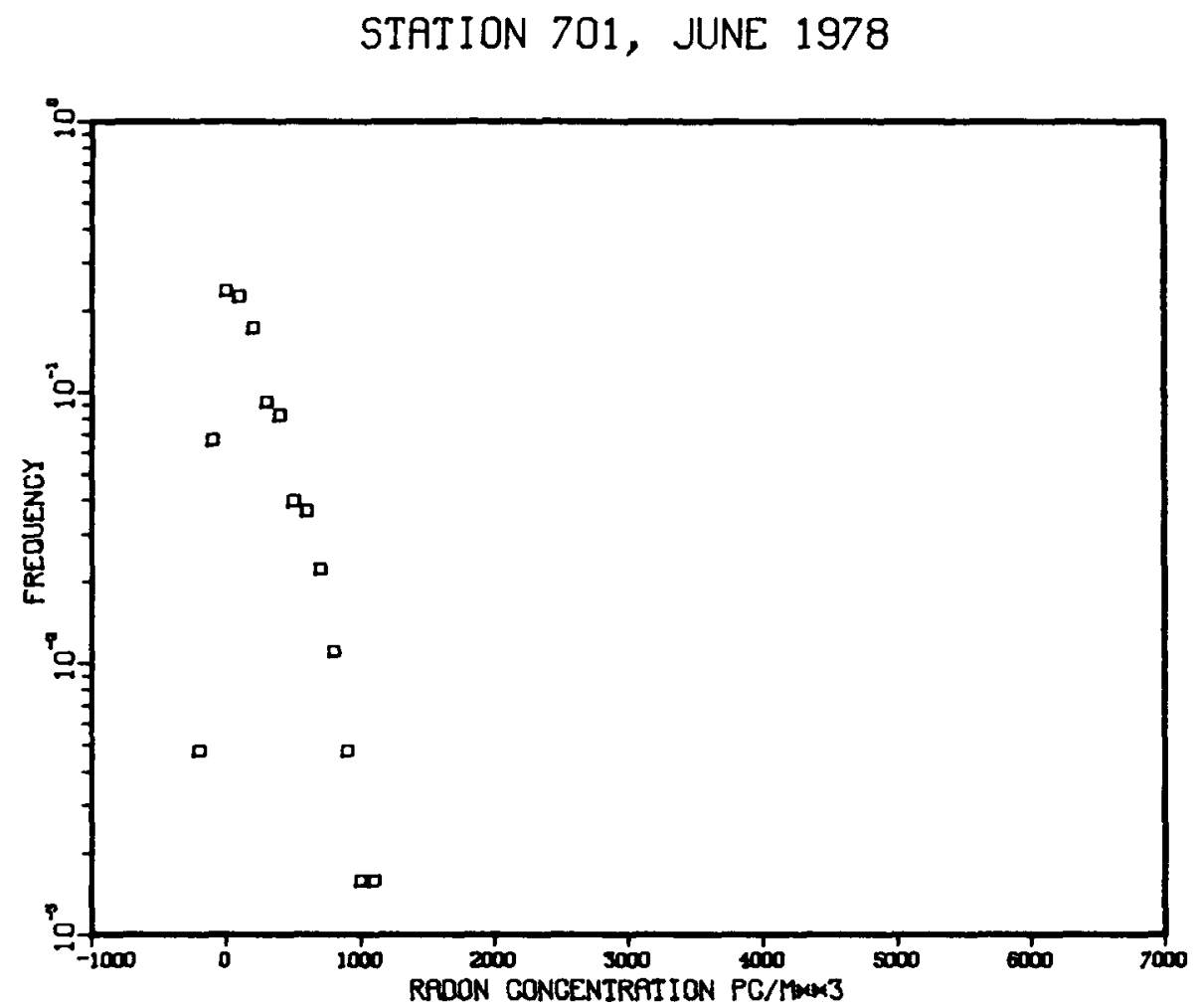

STATION 701, JULY 1978

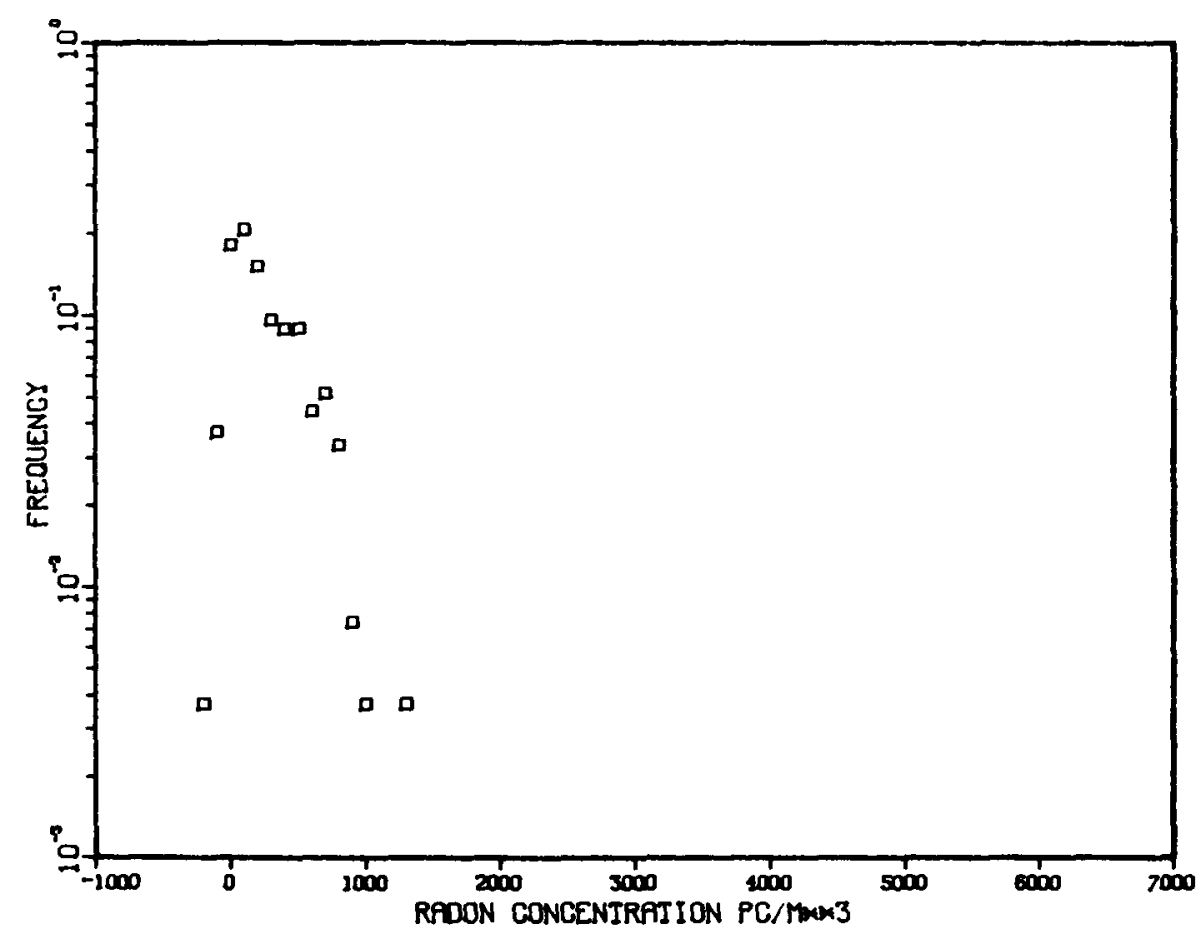

Figure A2. Continued. 
STATION 701, SEPTEMBER 1978

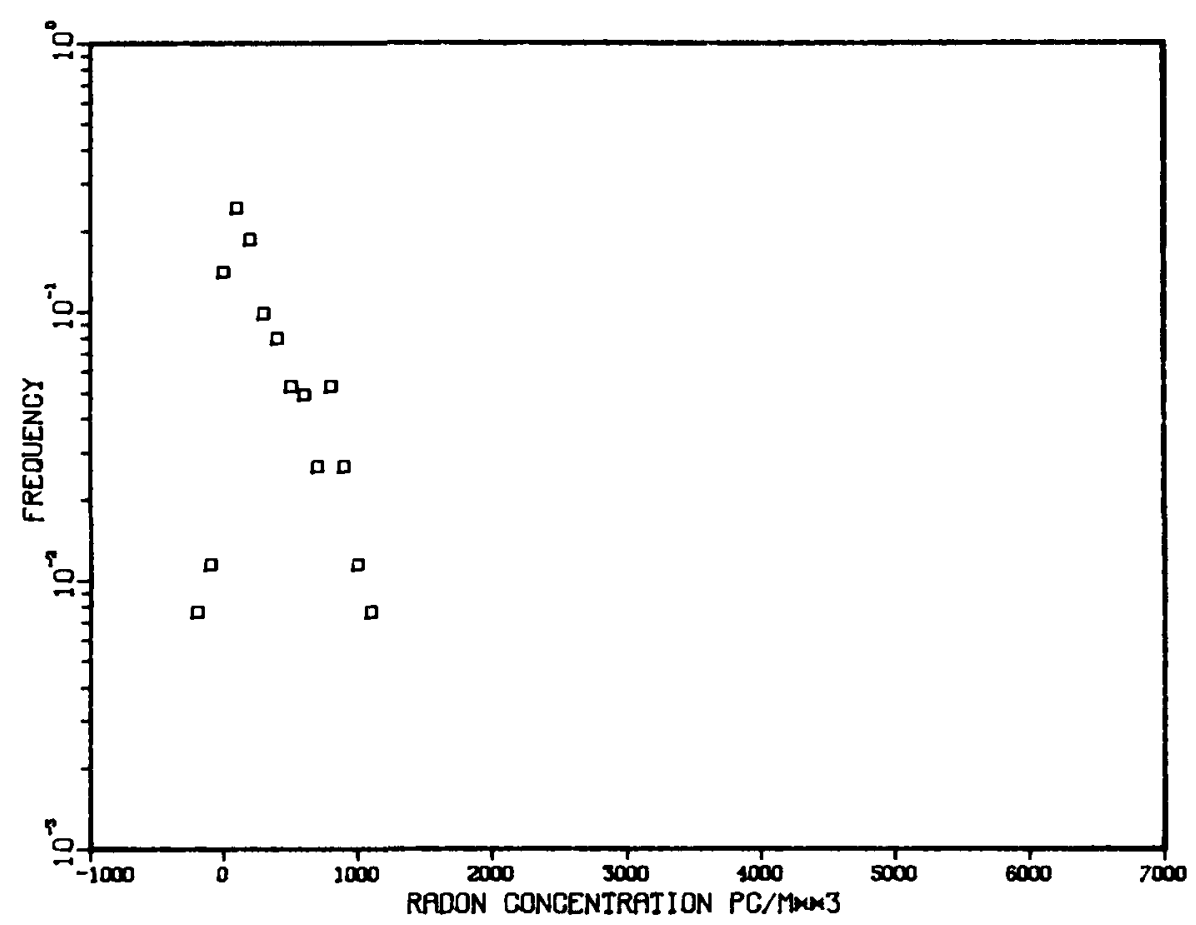

STATION 701, OCTOBER 1978

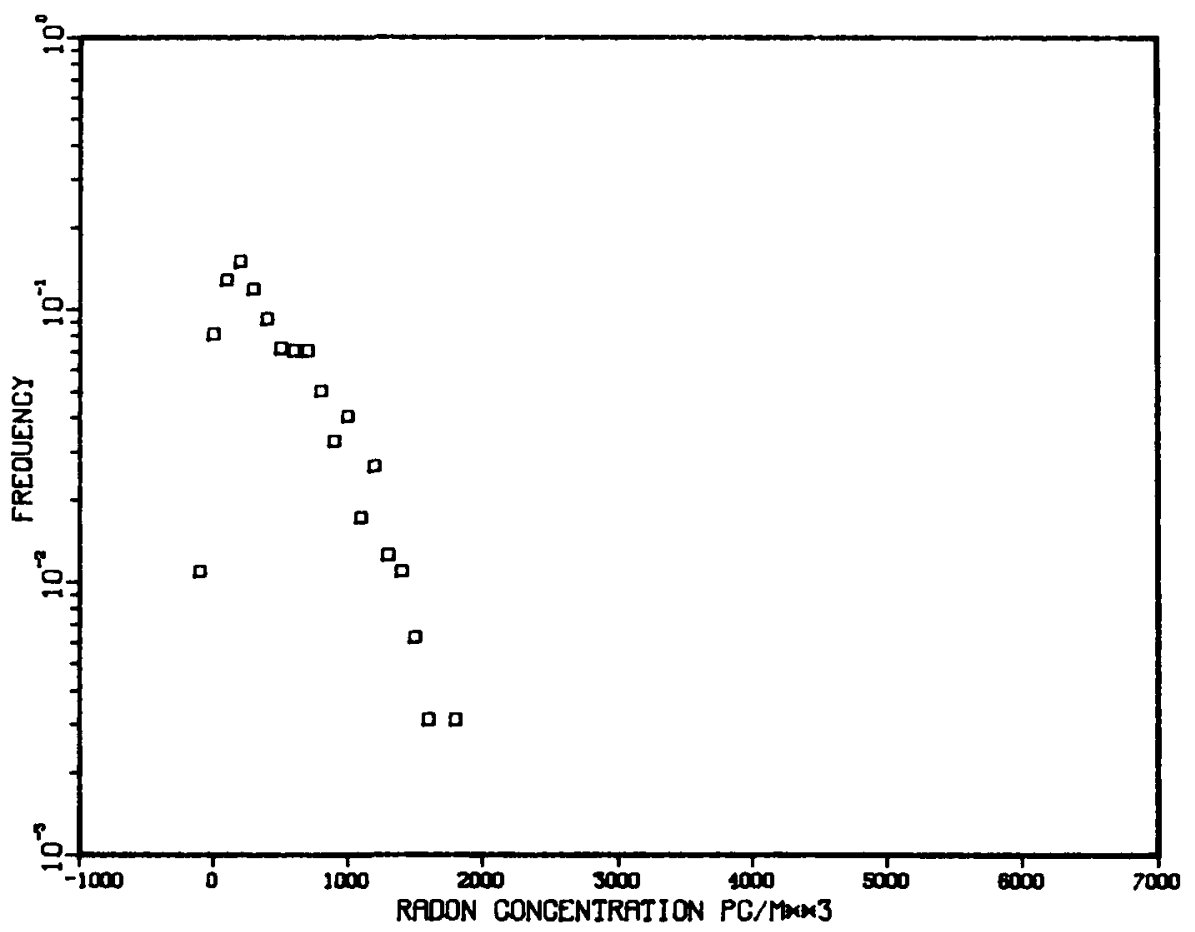

Figure A2. Continued. 


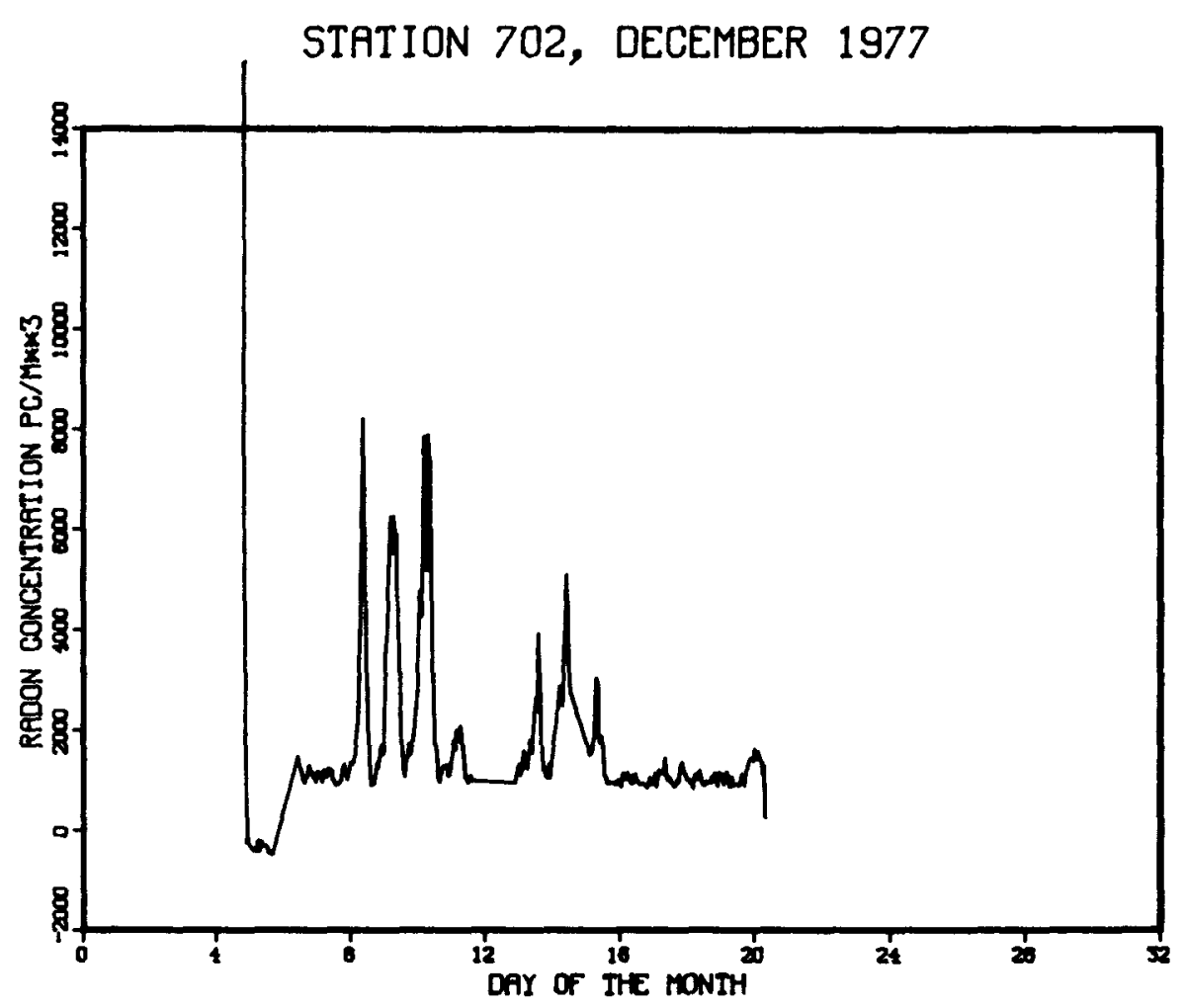

STATION 702, JANUARY 1978

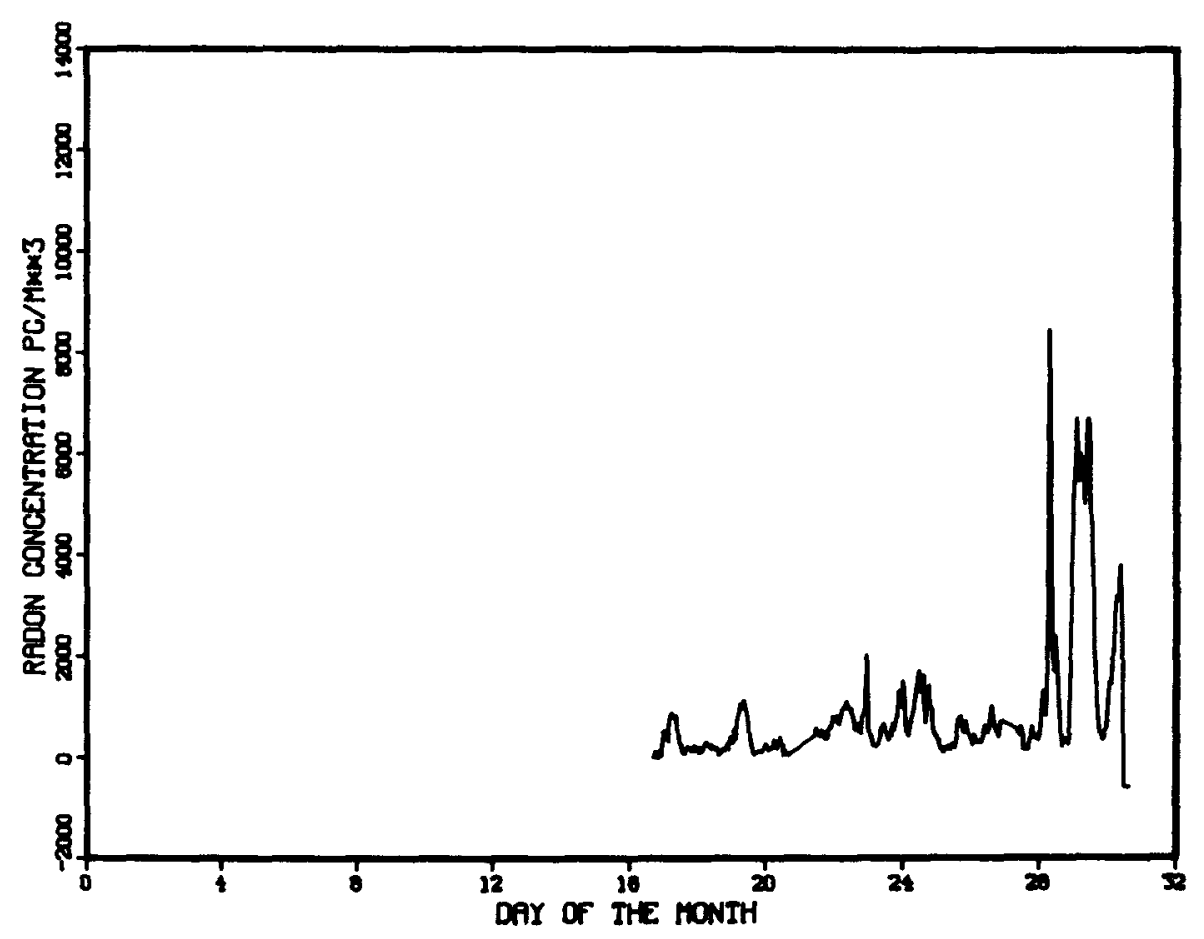

Figure A3. Radon Concentration by Hour at the McBride Background Station 702. 
STATION 702, APRIL 1978

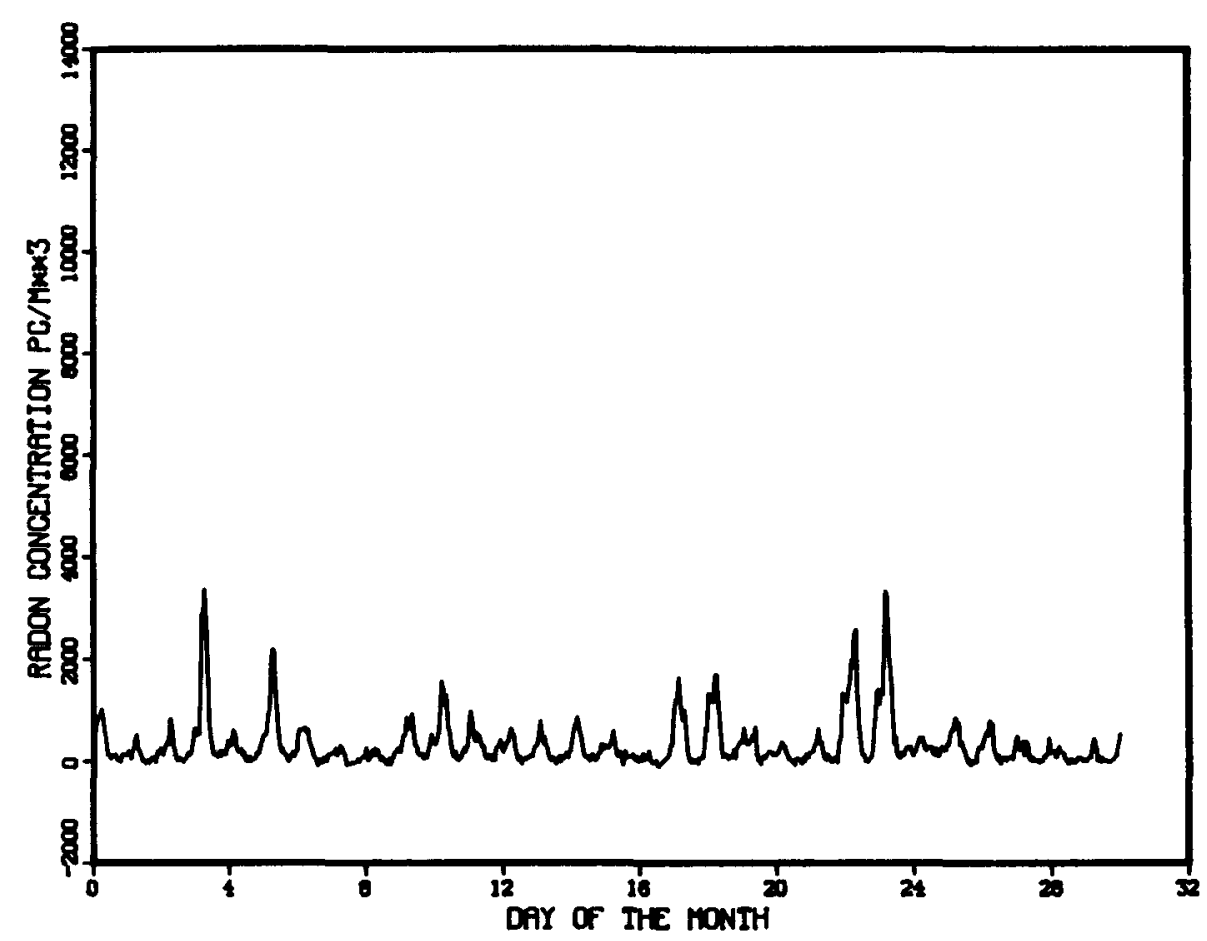

STATION 702, MAY 1978

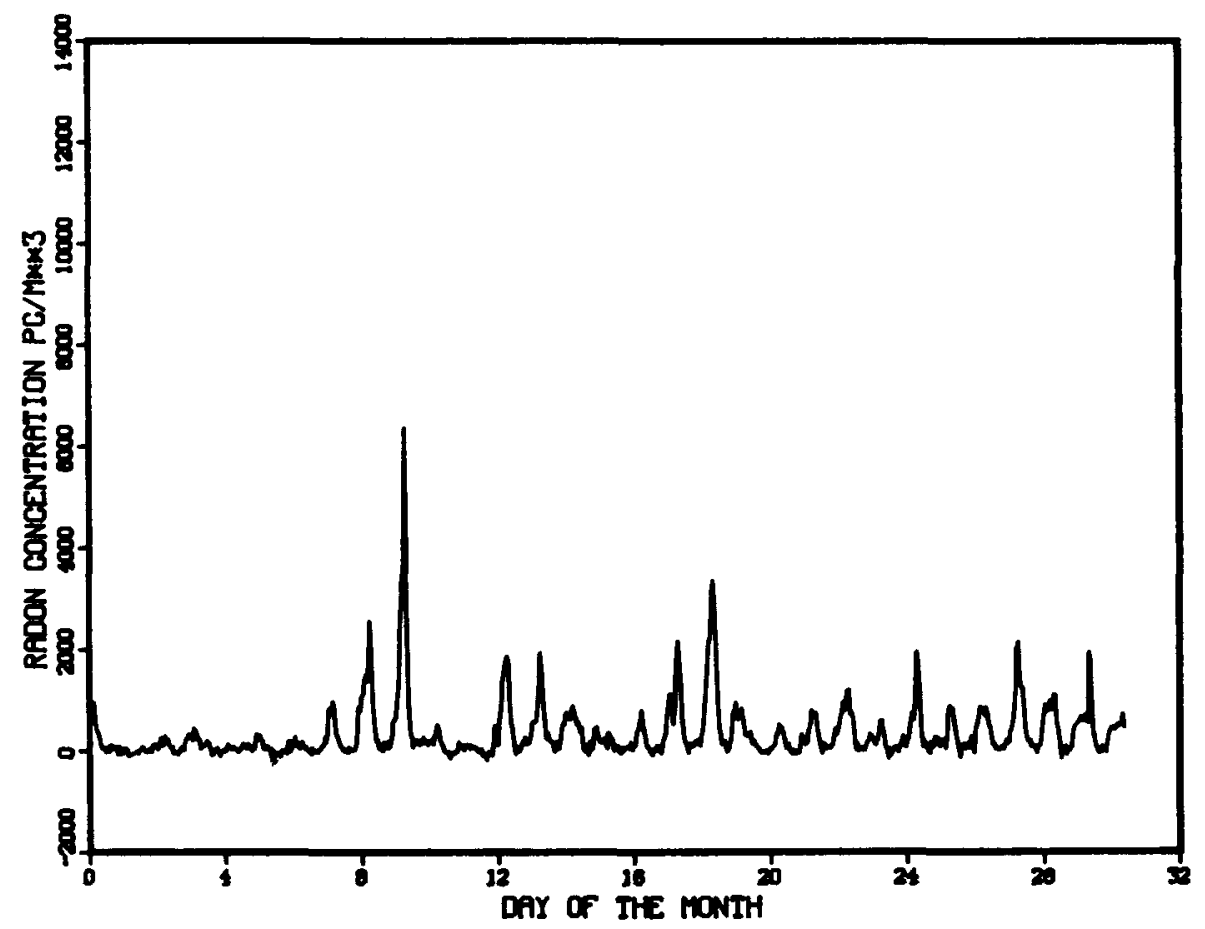

Figure A3. Continued. 
STATION 702, JUNE 1978

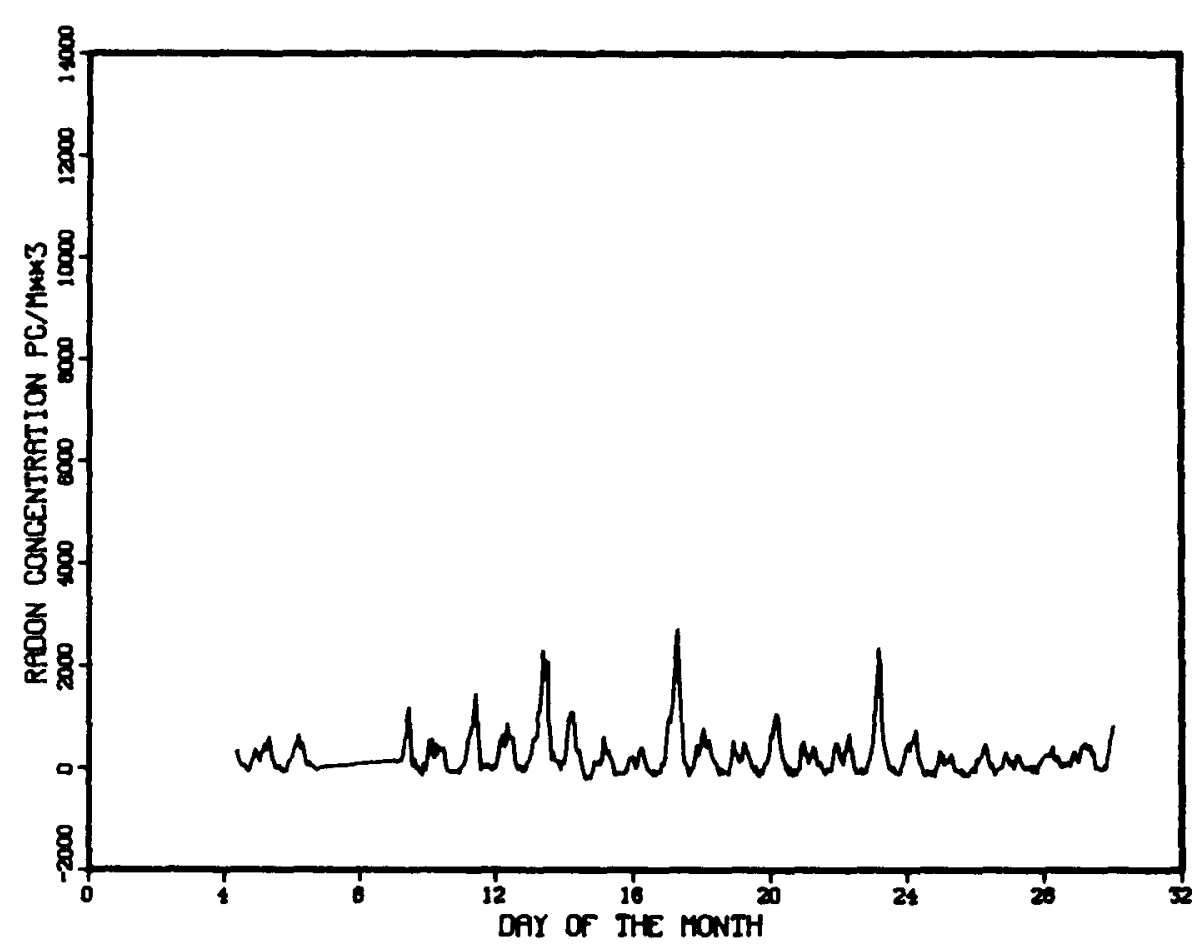

STATION 702, JULY 1978

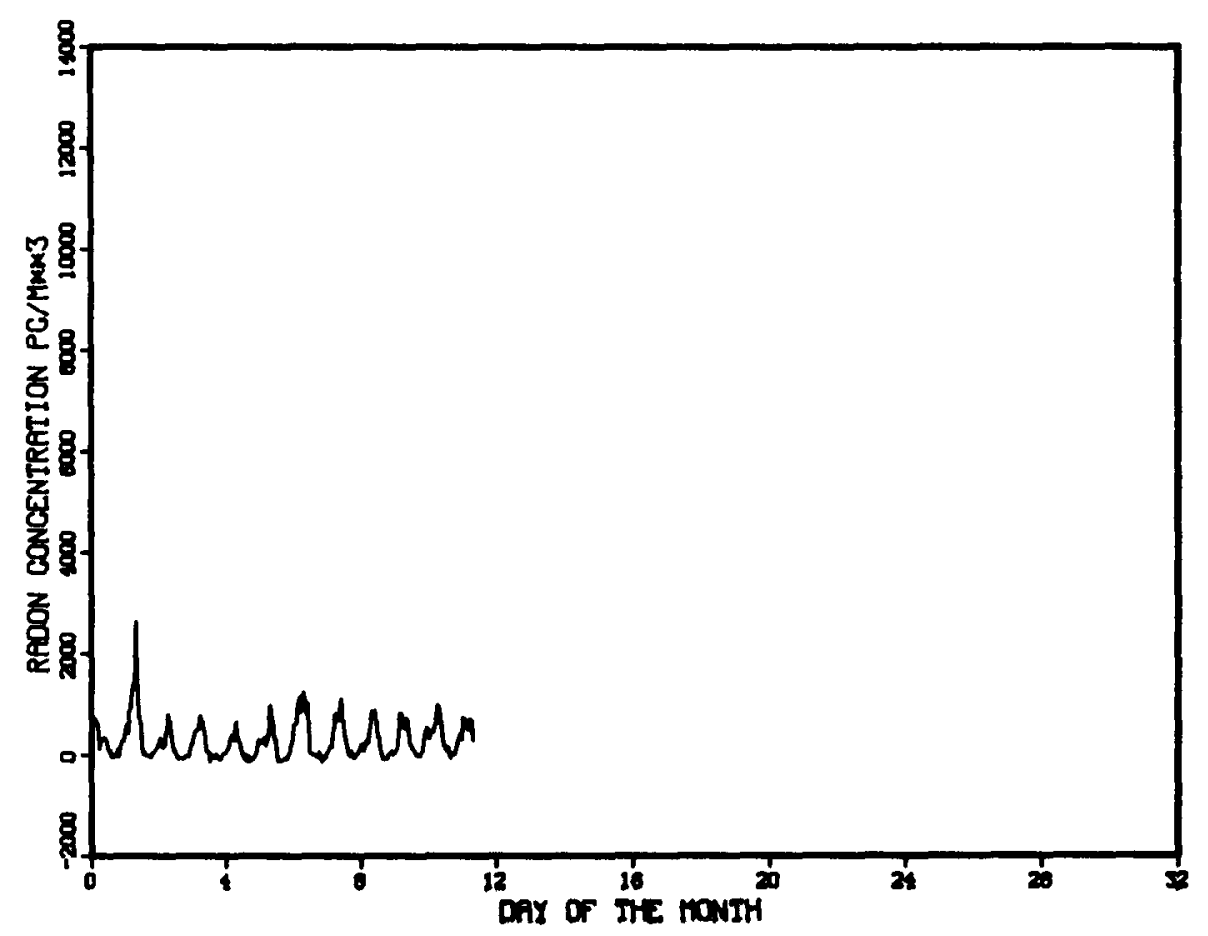

Figure A3. Continued. 
STATION 702, SEPTEMBER 1978

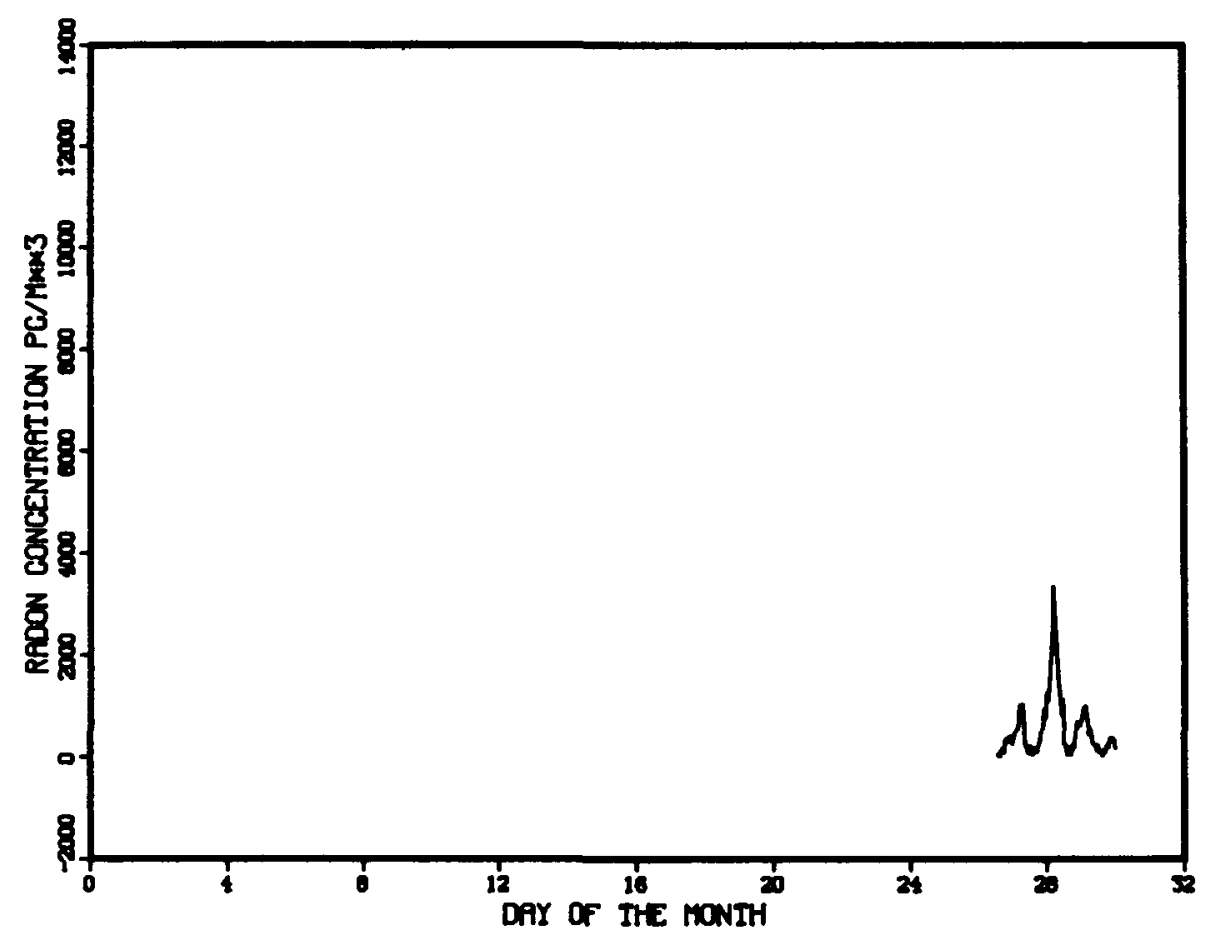

STATION 702, OCTOBER 1978

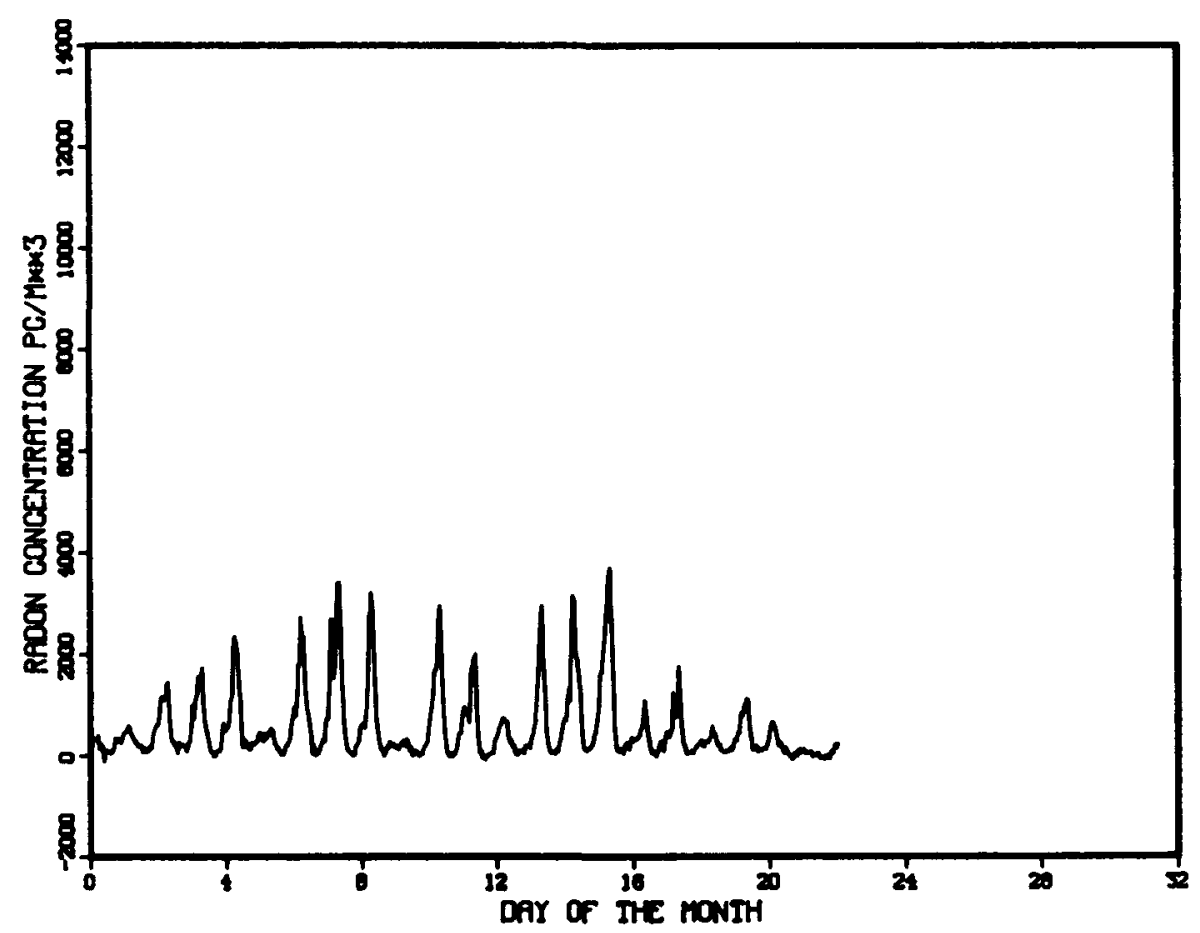

Figure A3. Continued. 
STATION 702, DECEMBER 1977

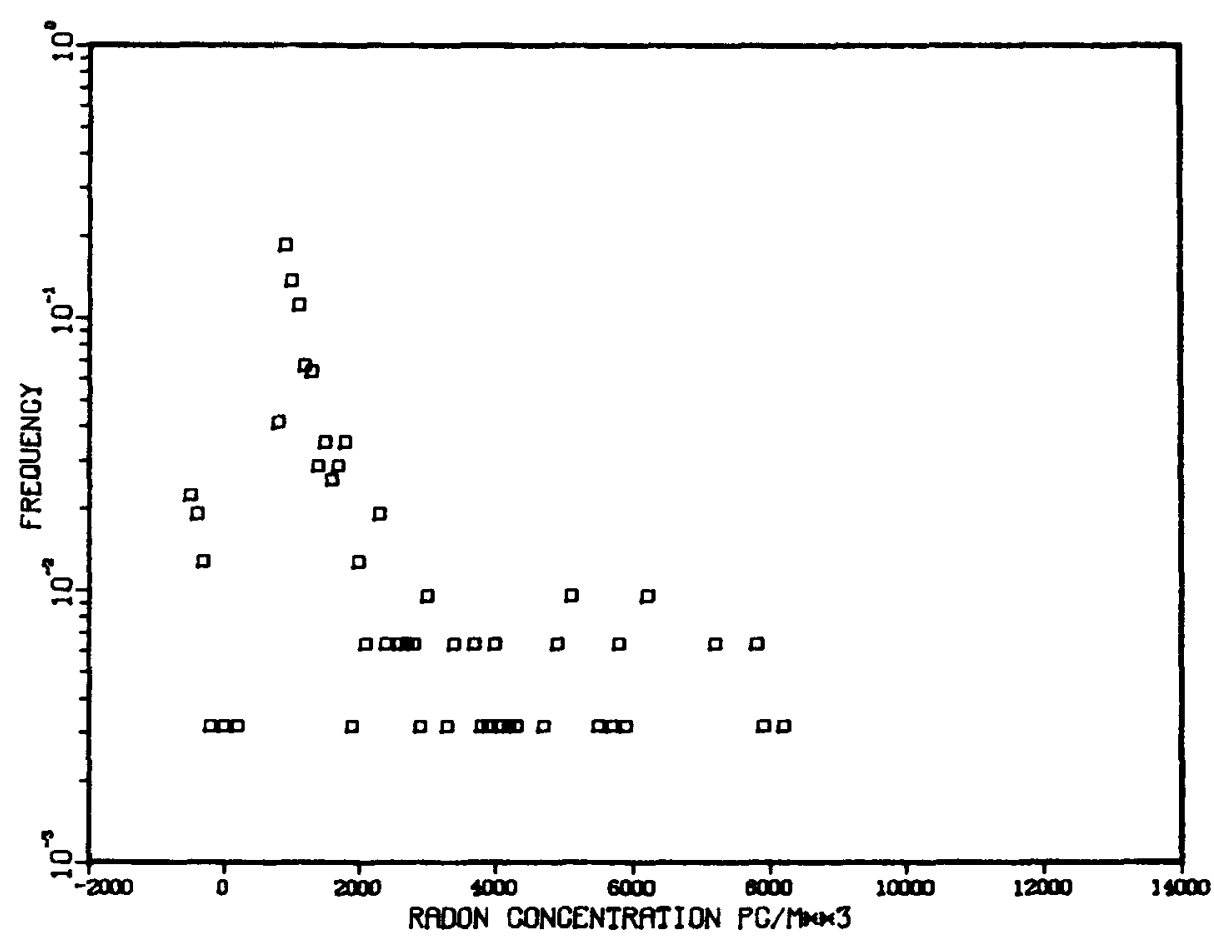

STATION 702, JANUARY 1978

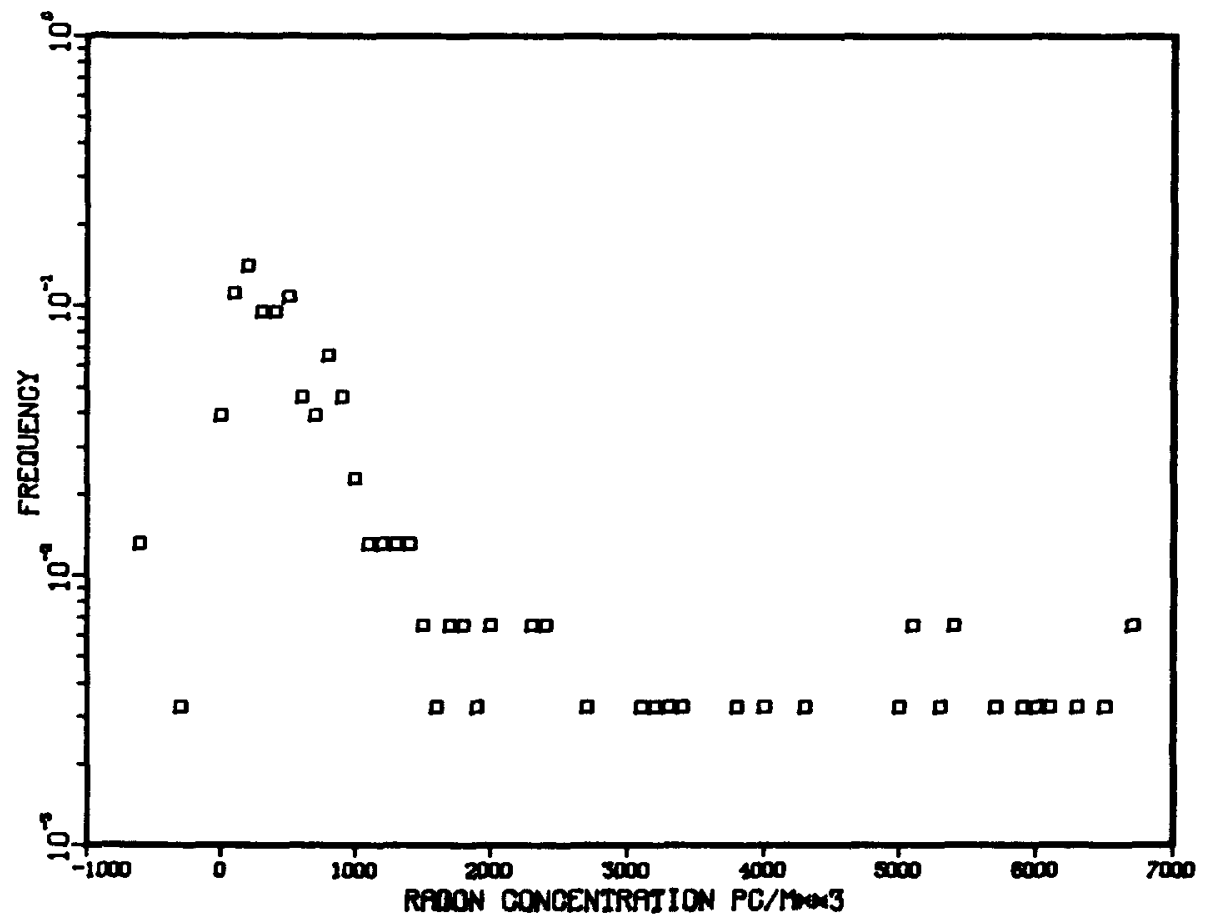

Figure A4. Radon-Concentration Frequency at the McBride Background Station 702 . 
STATION 702, APRIL 1978

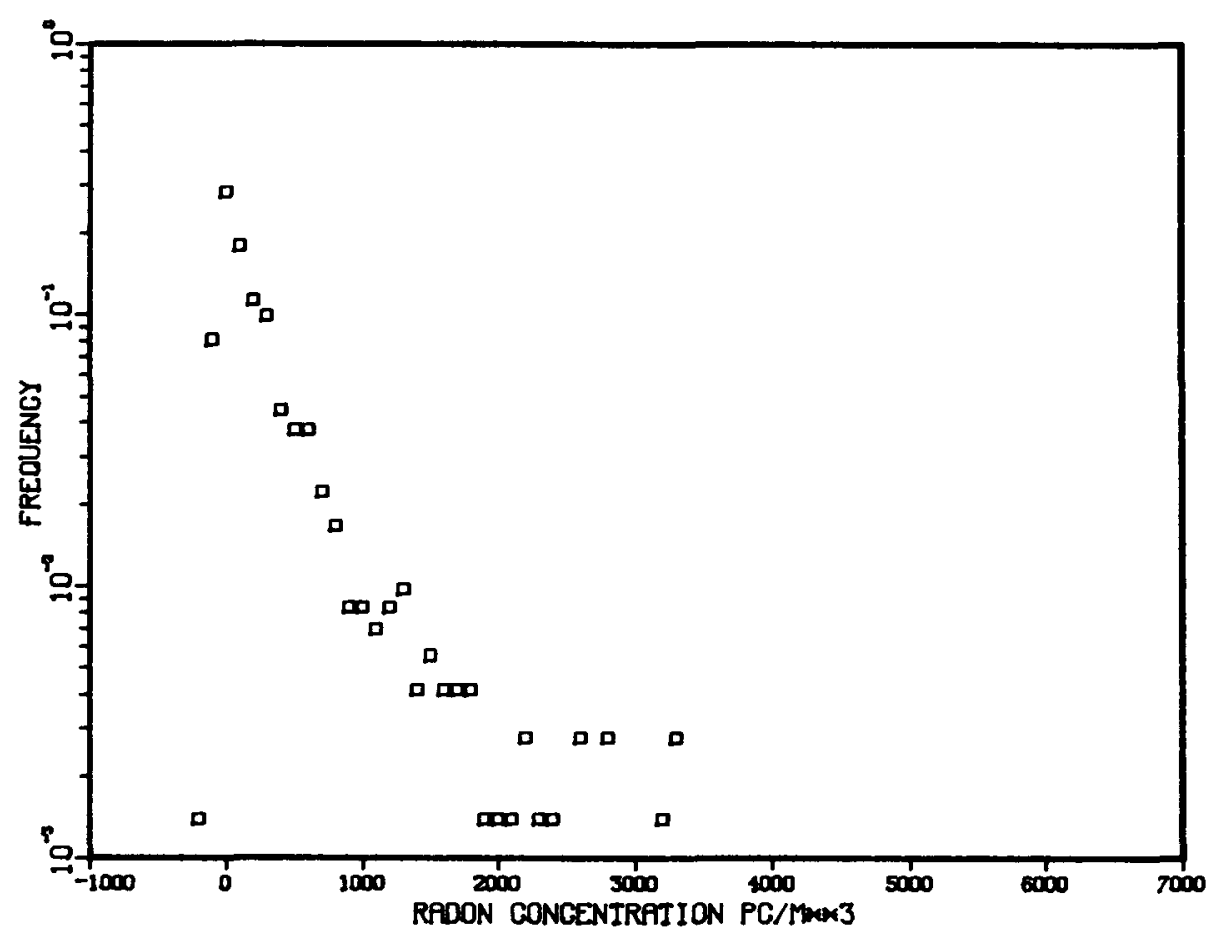

STATION 702, MAY 1978

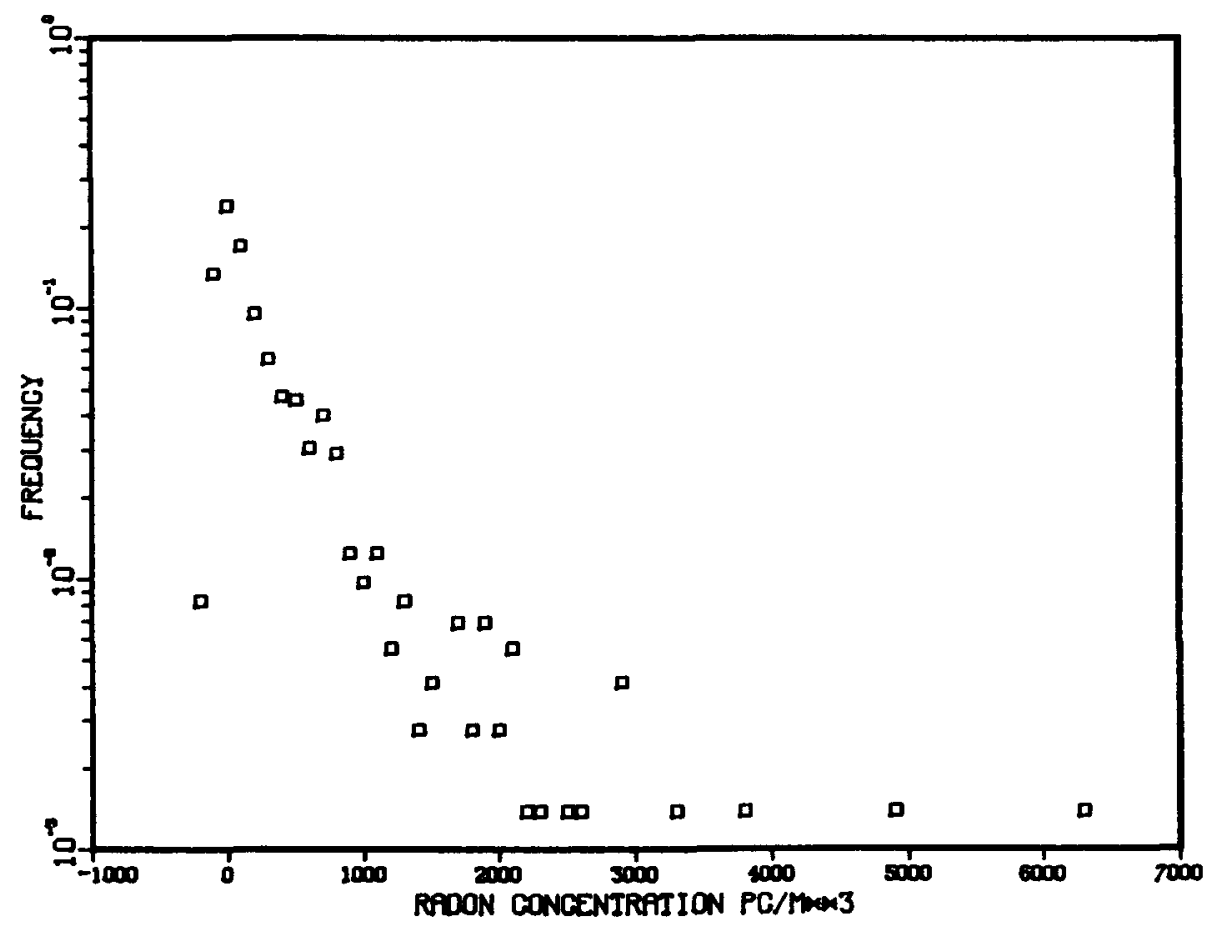

Figure A4. Continued. 
STATION 702, JUNE 1978

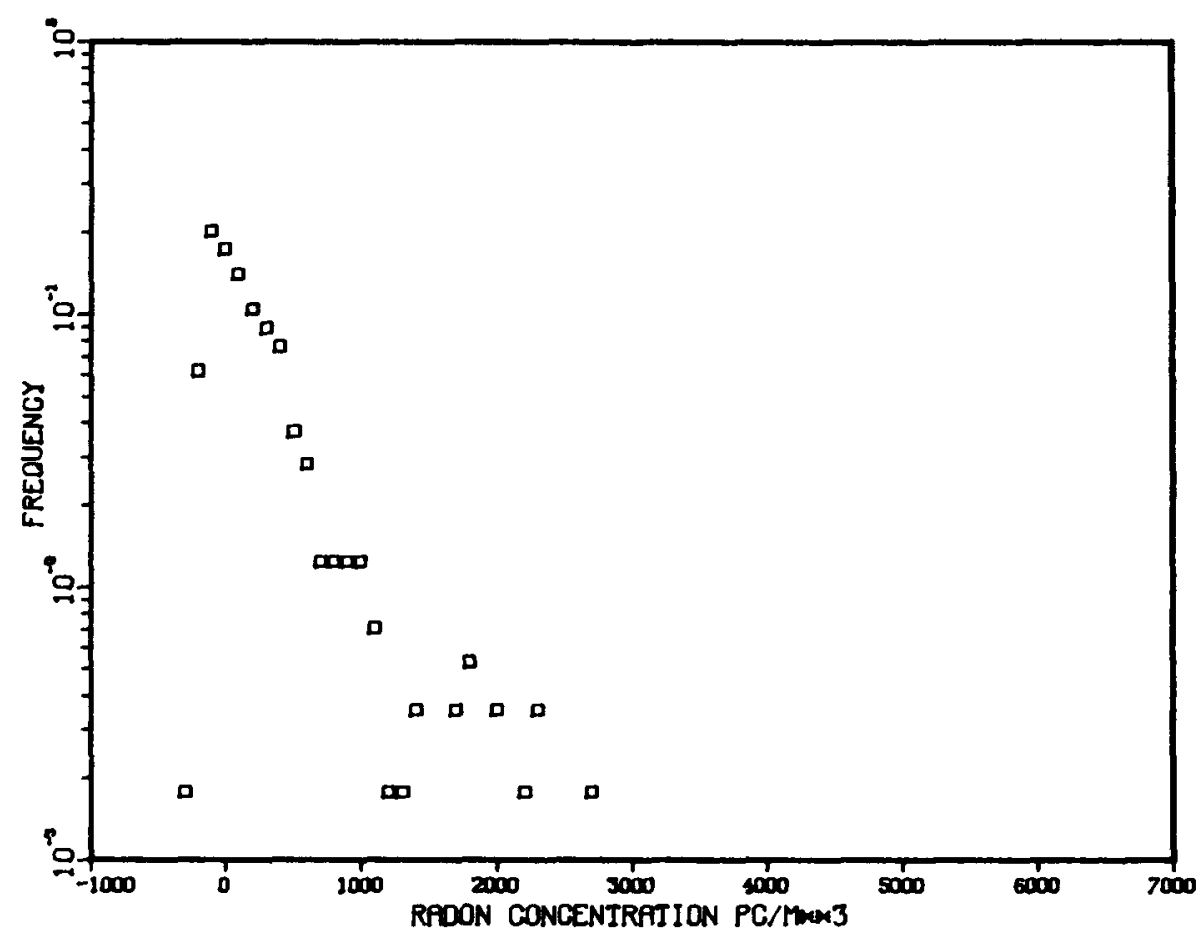

STATION 702, JULY 1978

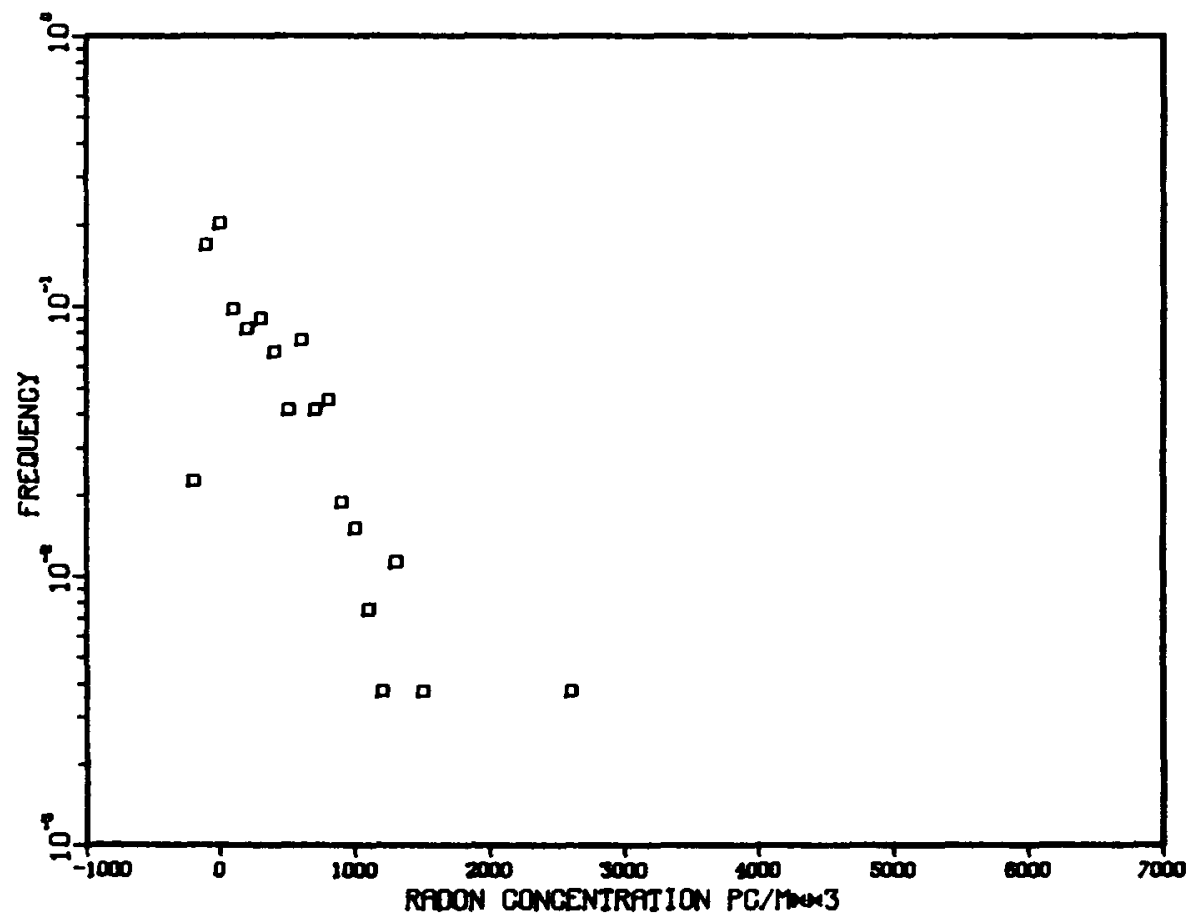

Figure A4. Continued. 
STATION 702, SEPTEMBER 1978

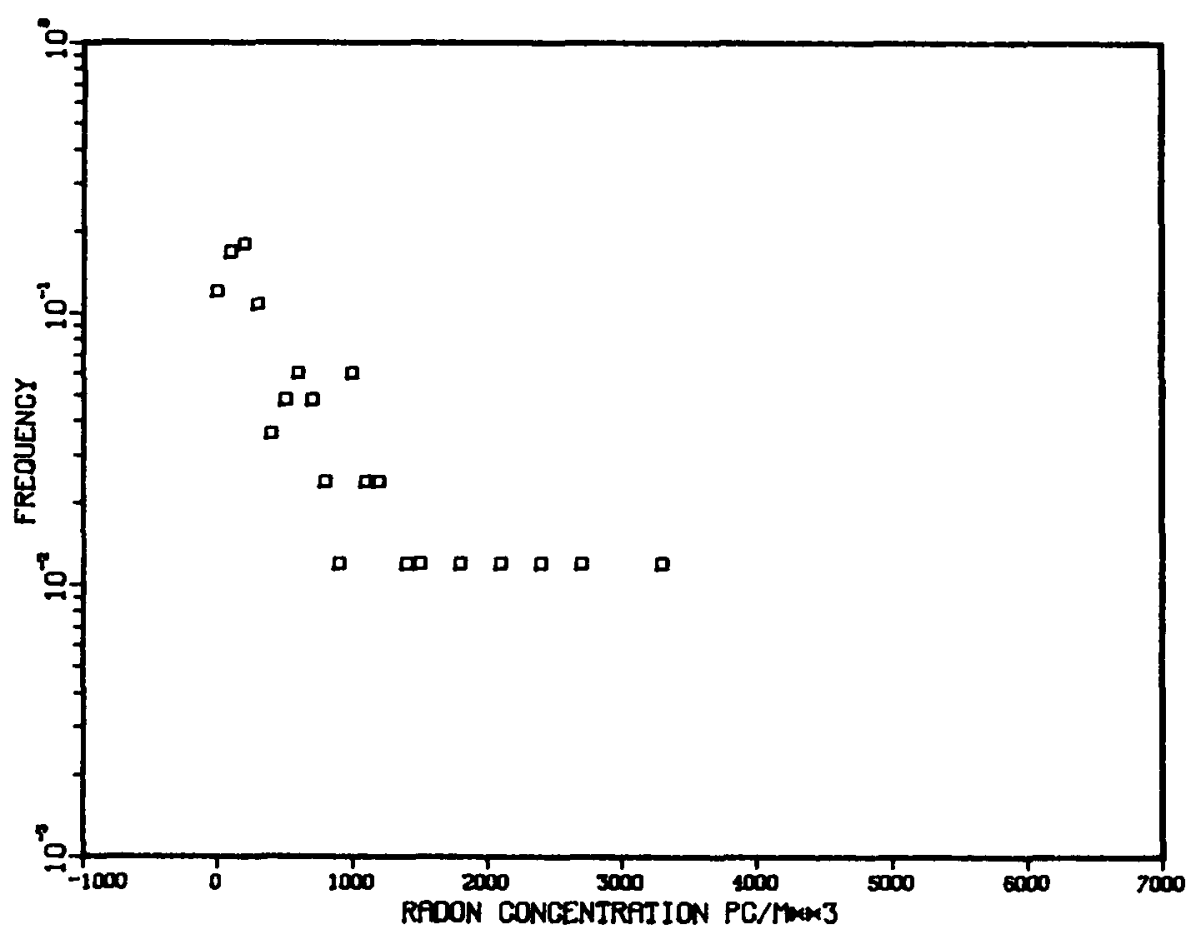

STATION 702, OCTOBER 1978

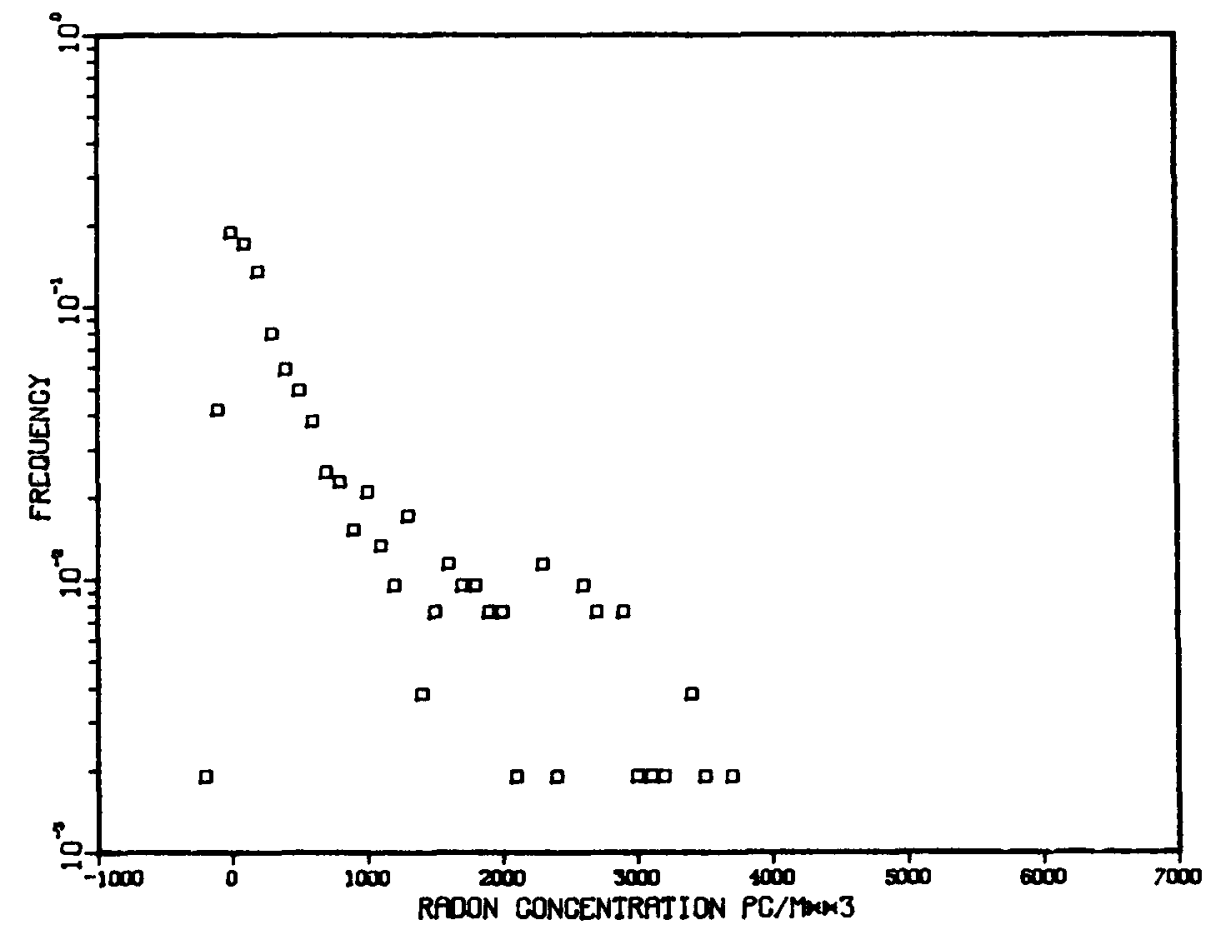

Figure A4. Continued. 
STATION 102, JULY 1977

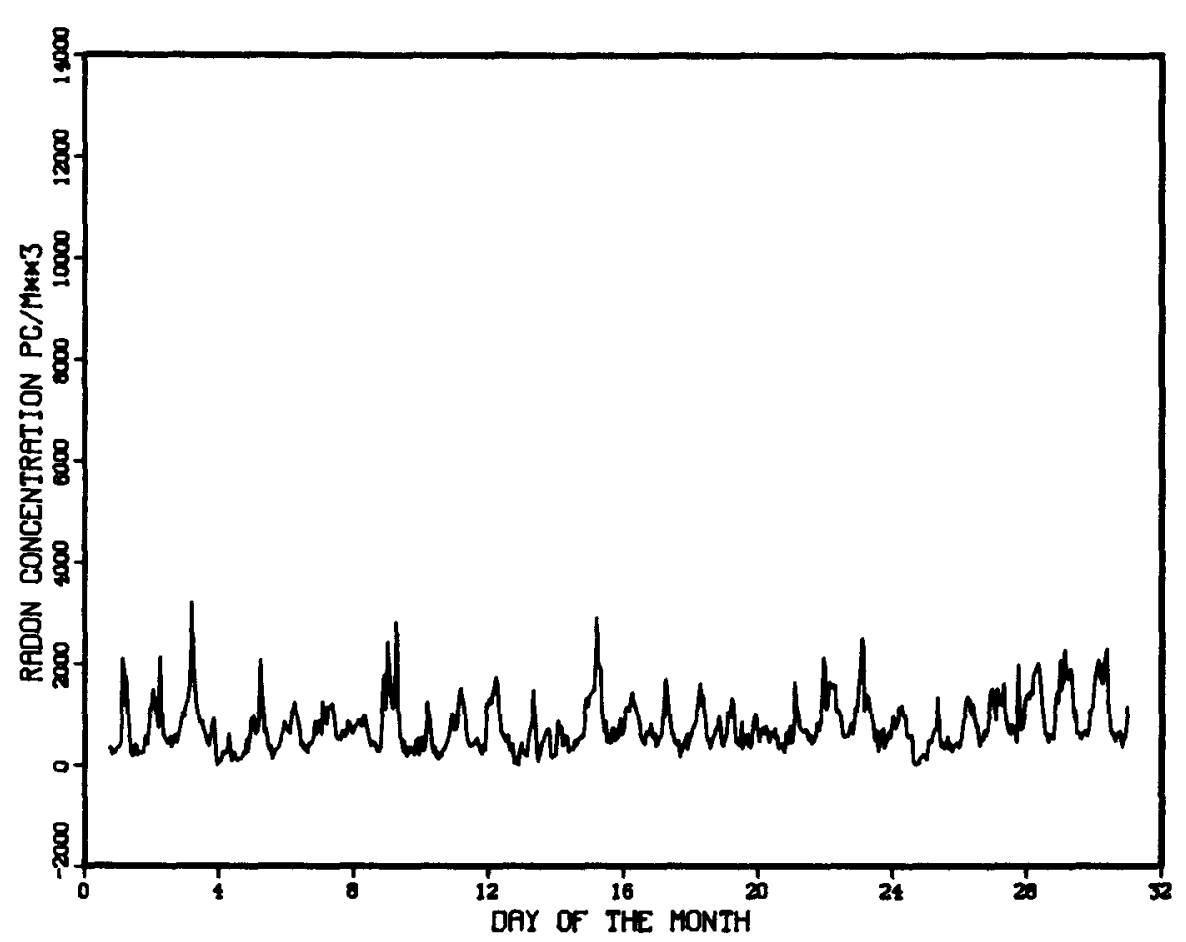

STATION 102, AUGUST 1977

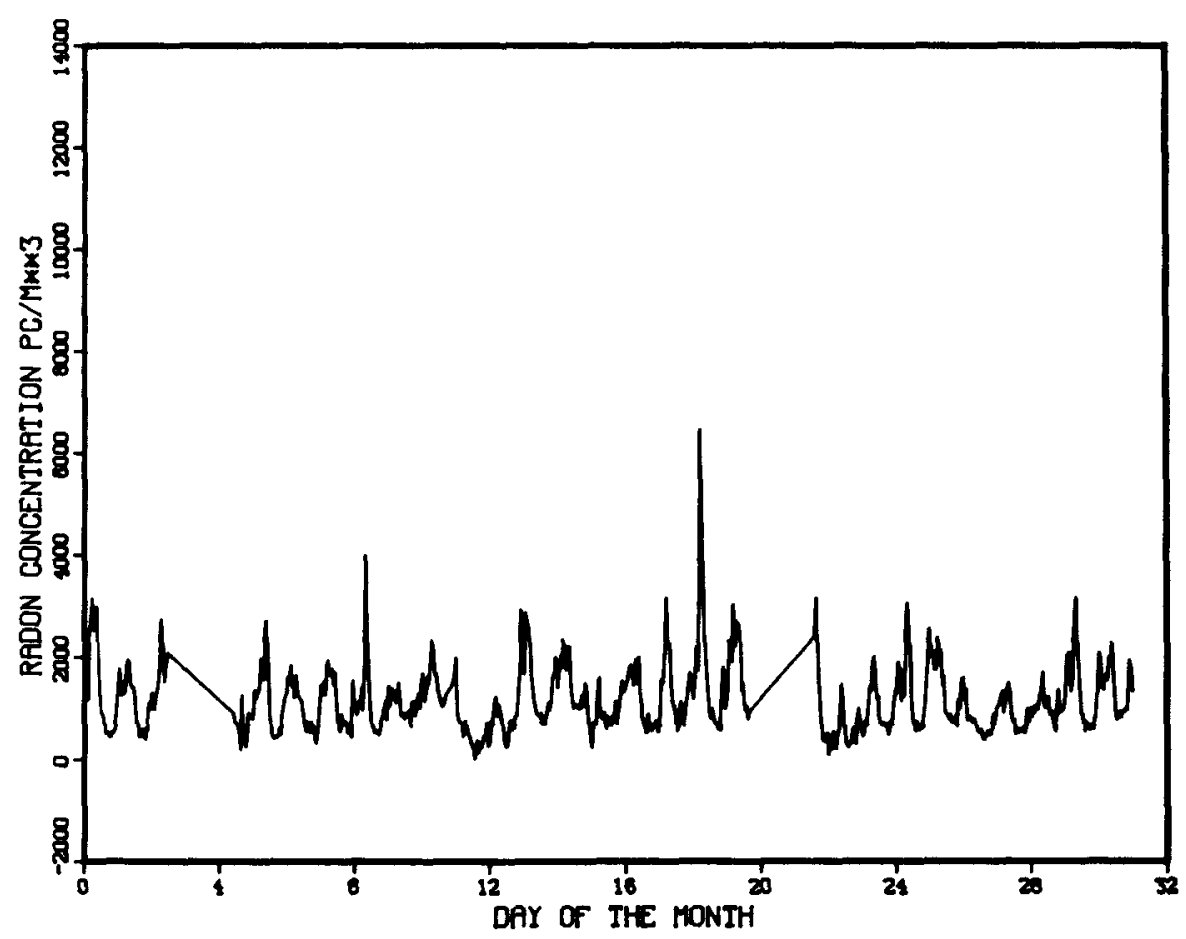

Figure A5. Radon Concentration by Hour at Station 102. 
STATION 102, SEPTEMBER 1977

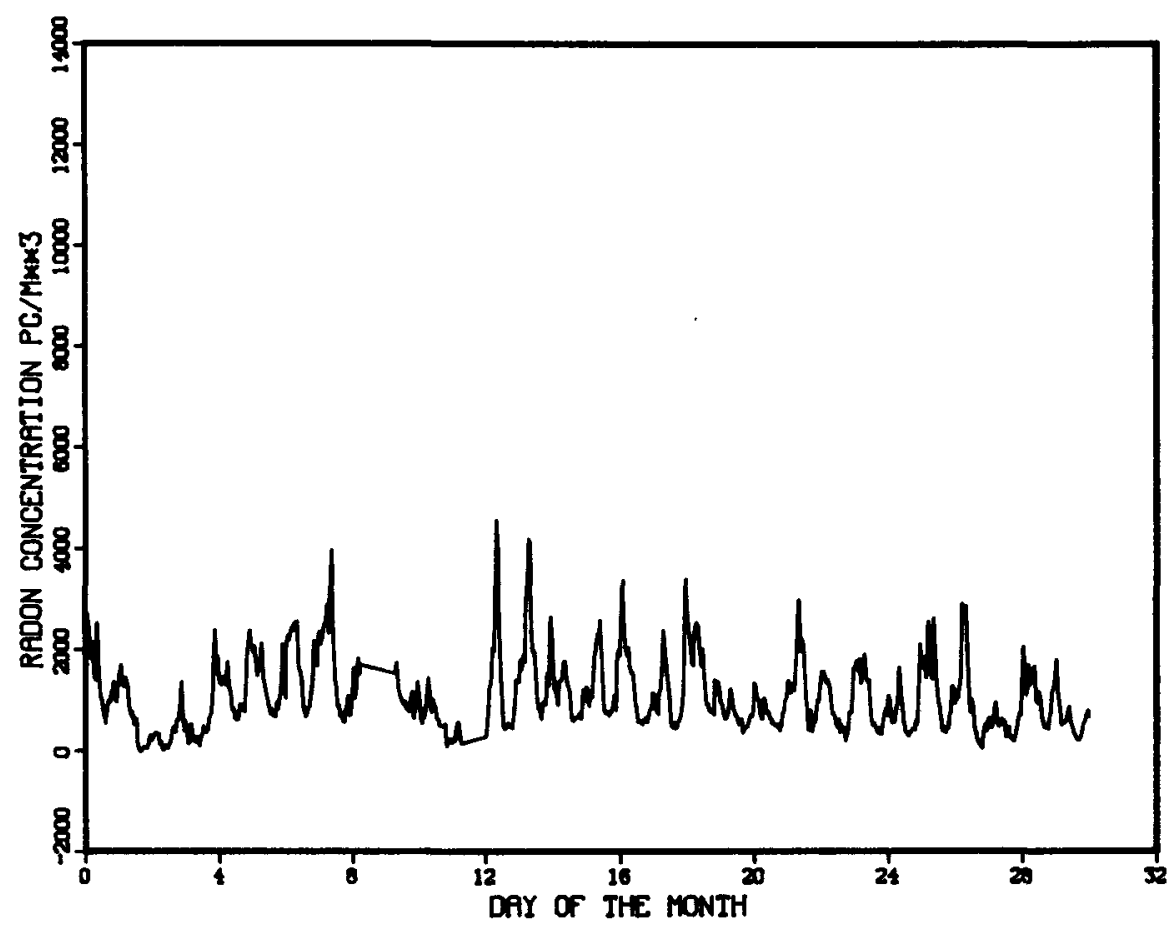

STATION 102, OCTOBER 1977

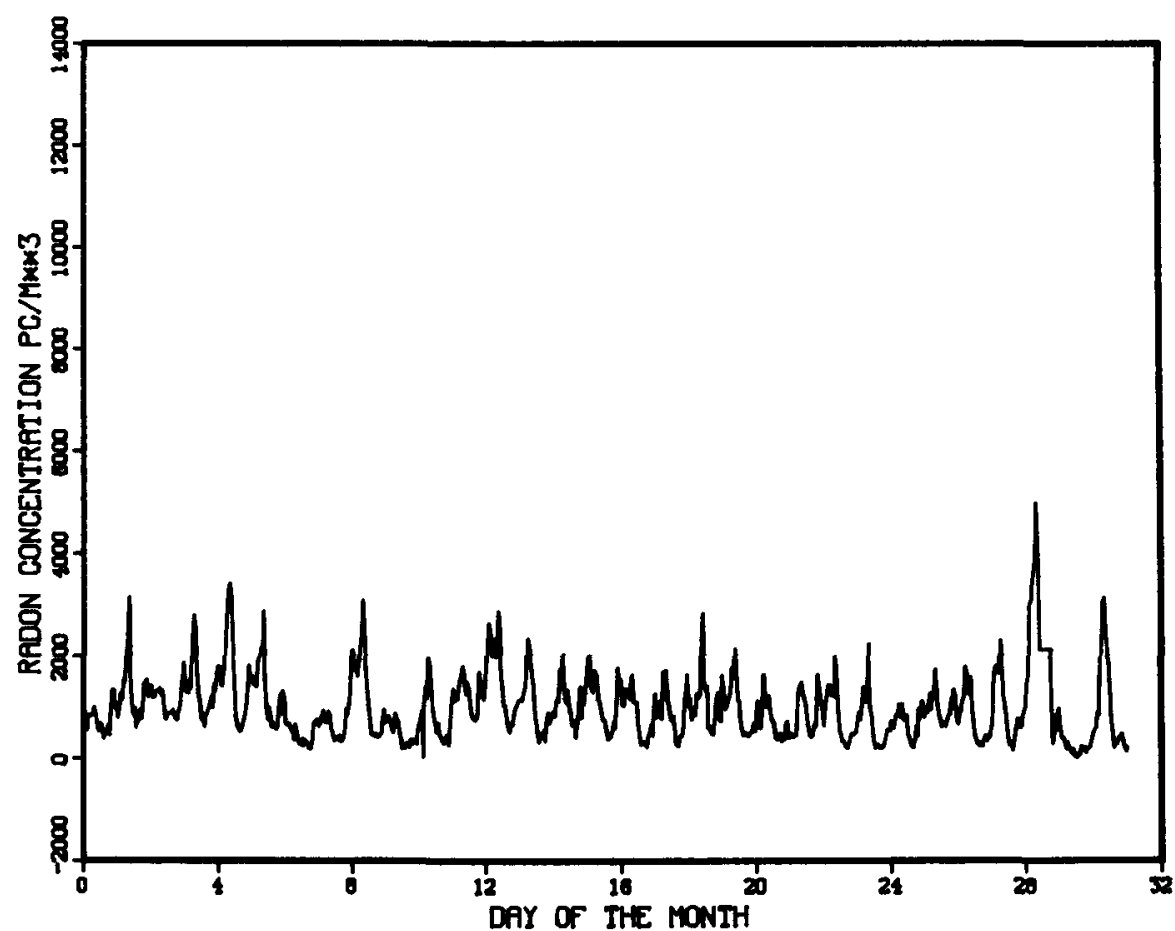

Figure A5. Continued. 
STATION 102, NOVEMBER 1977

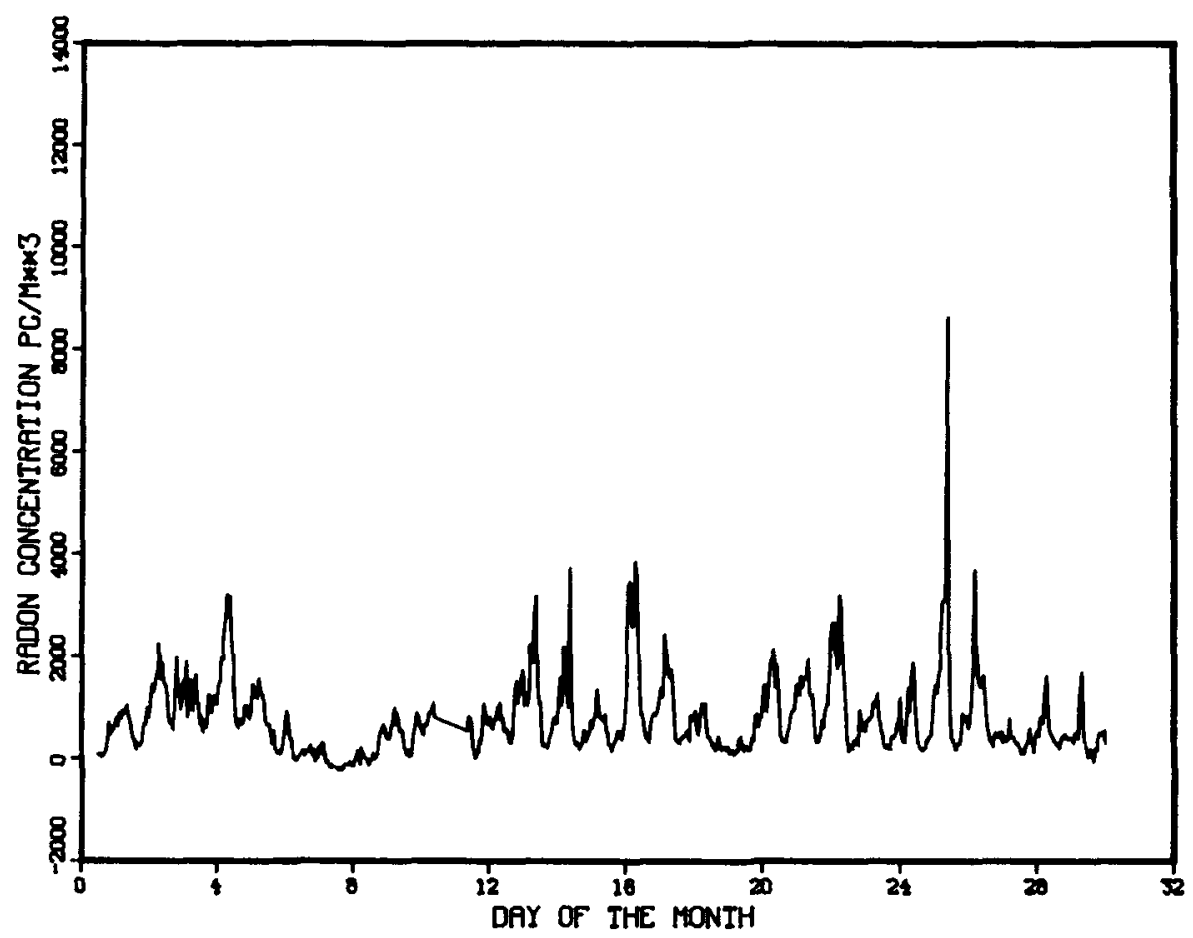

STATION 102, DECEMBER 1977

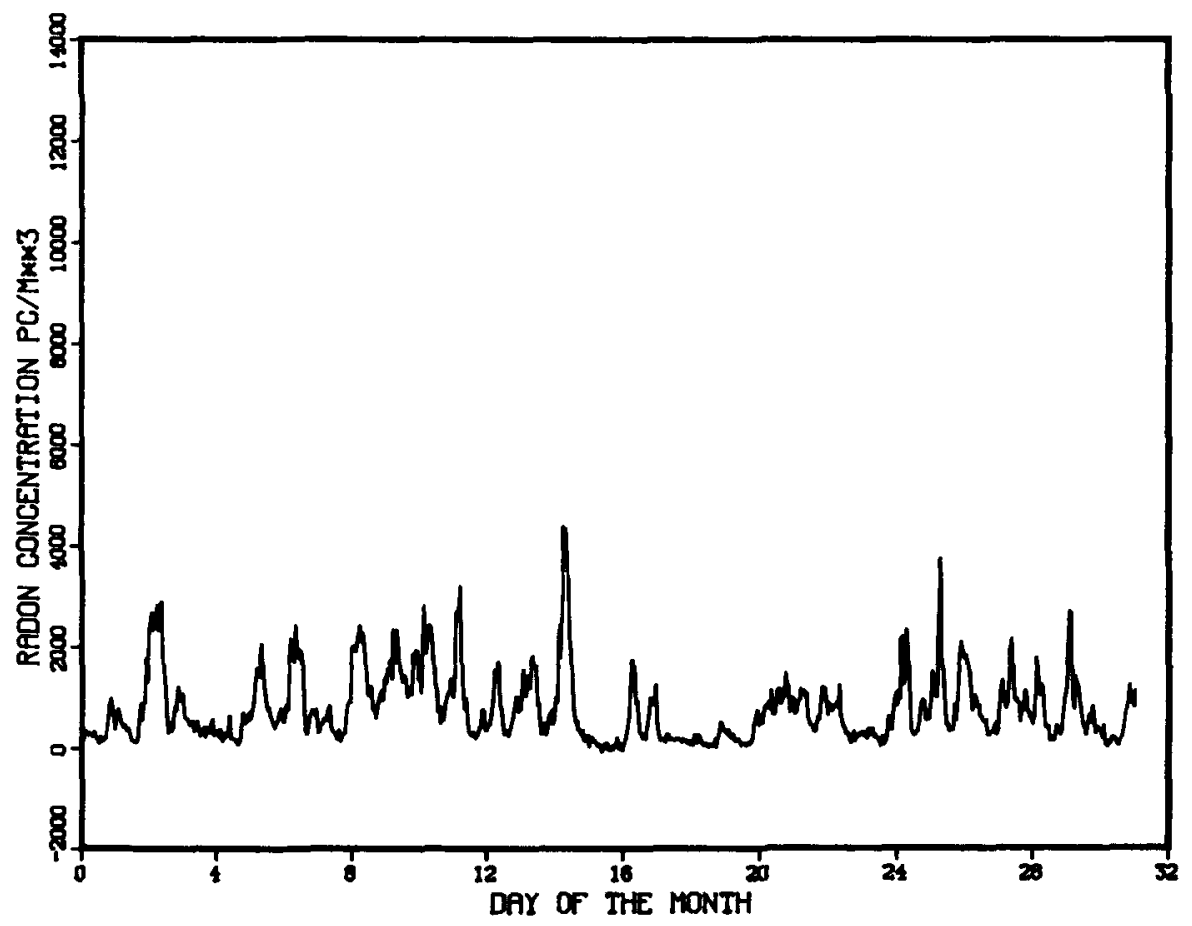

Figure A5. Continued. 
STATION 102, MARCH 1978

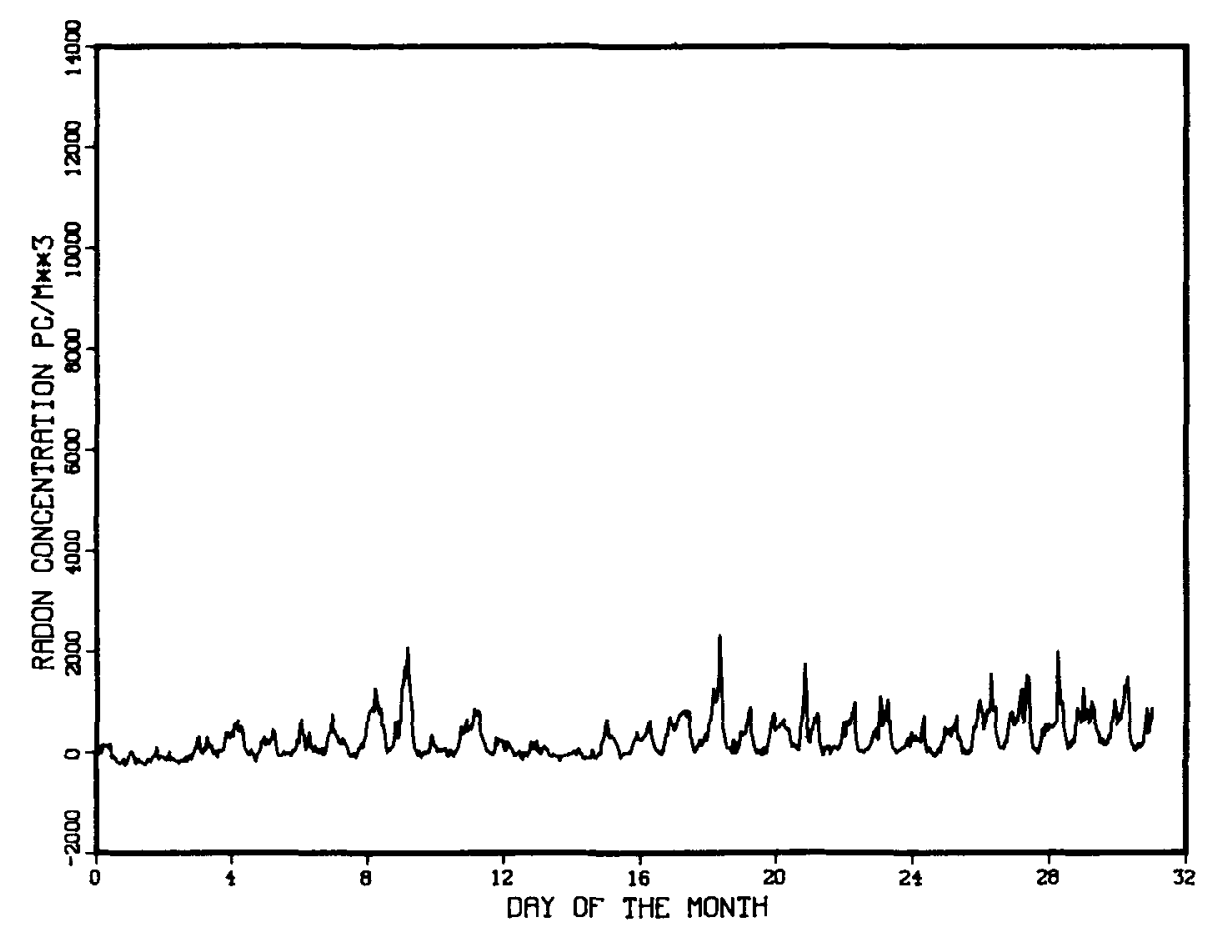

STATION 102, APRIL 1978

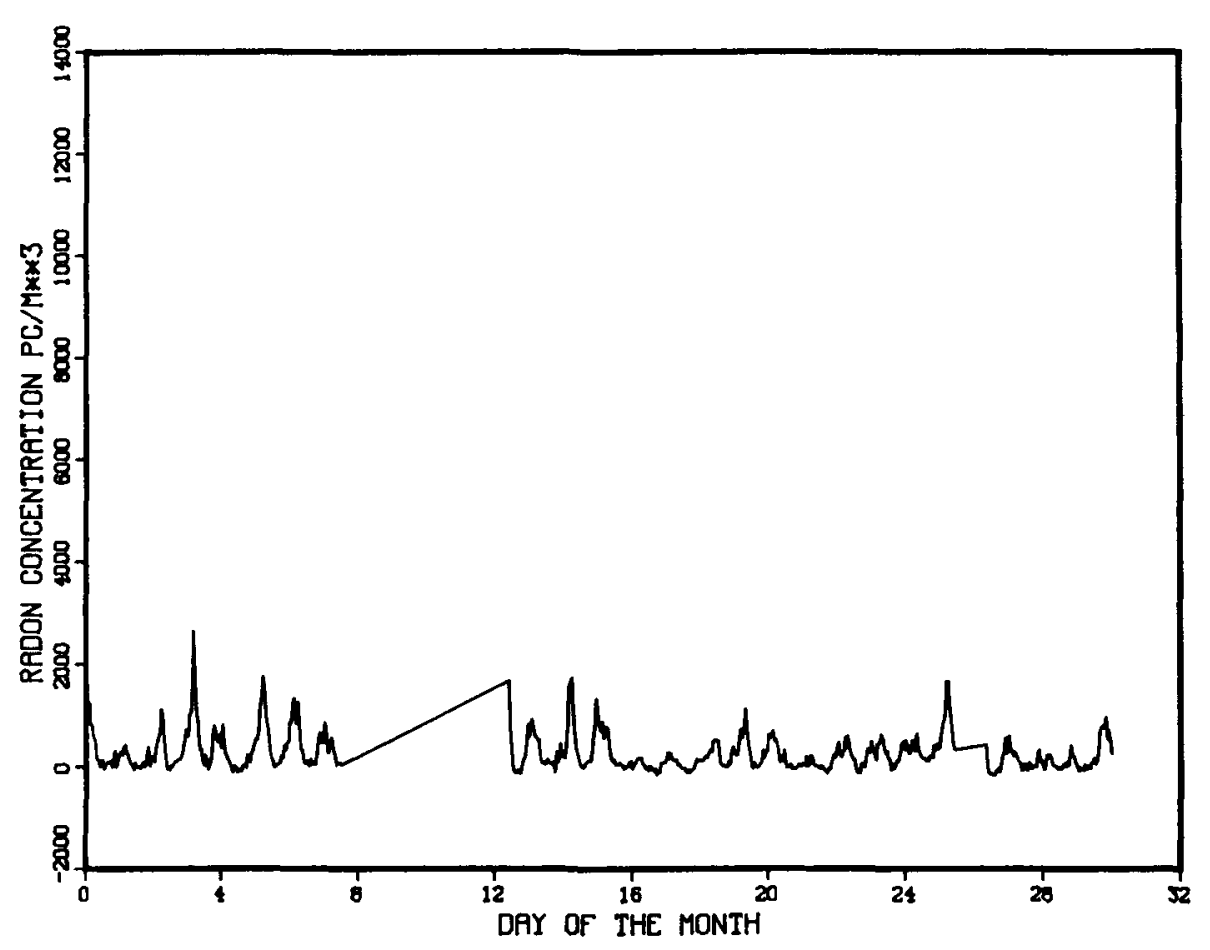

Figure A5. Continued. 

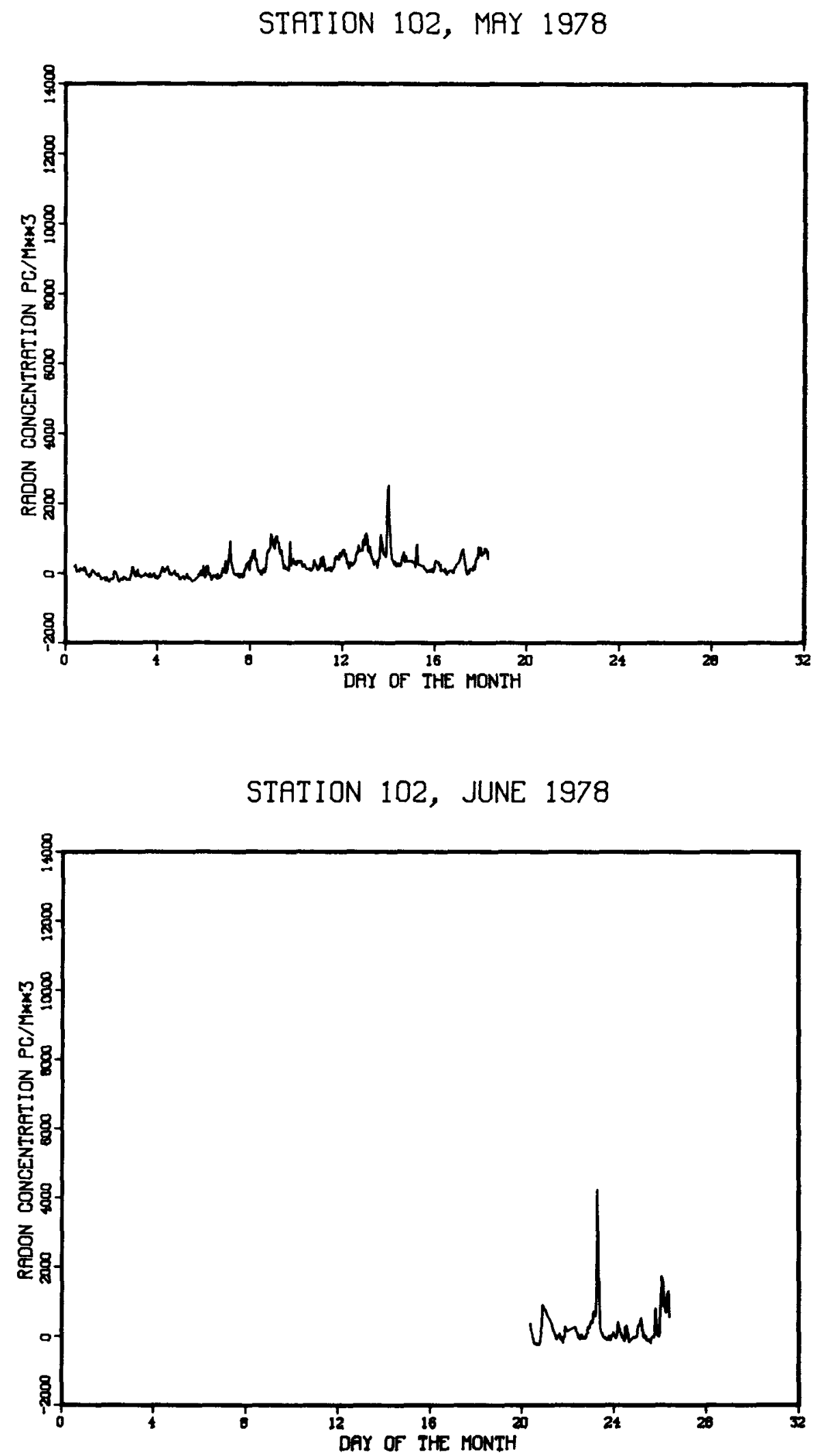

Figure A5. Continued. 
STATION 103, JULY 1977
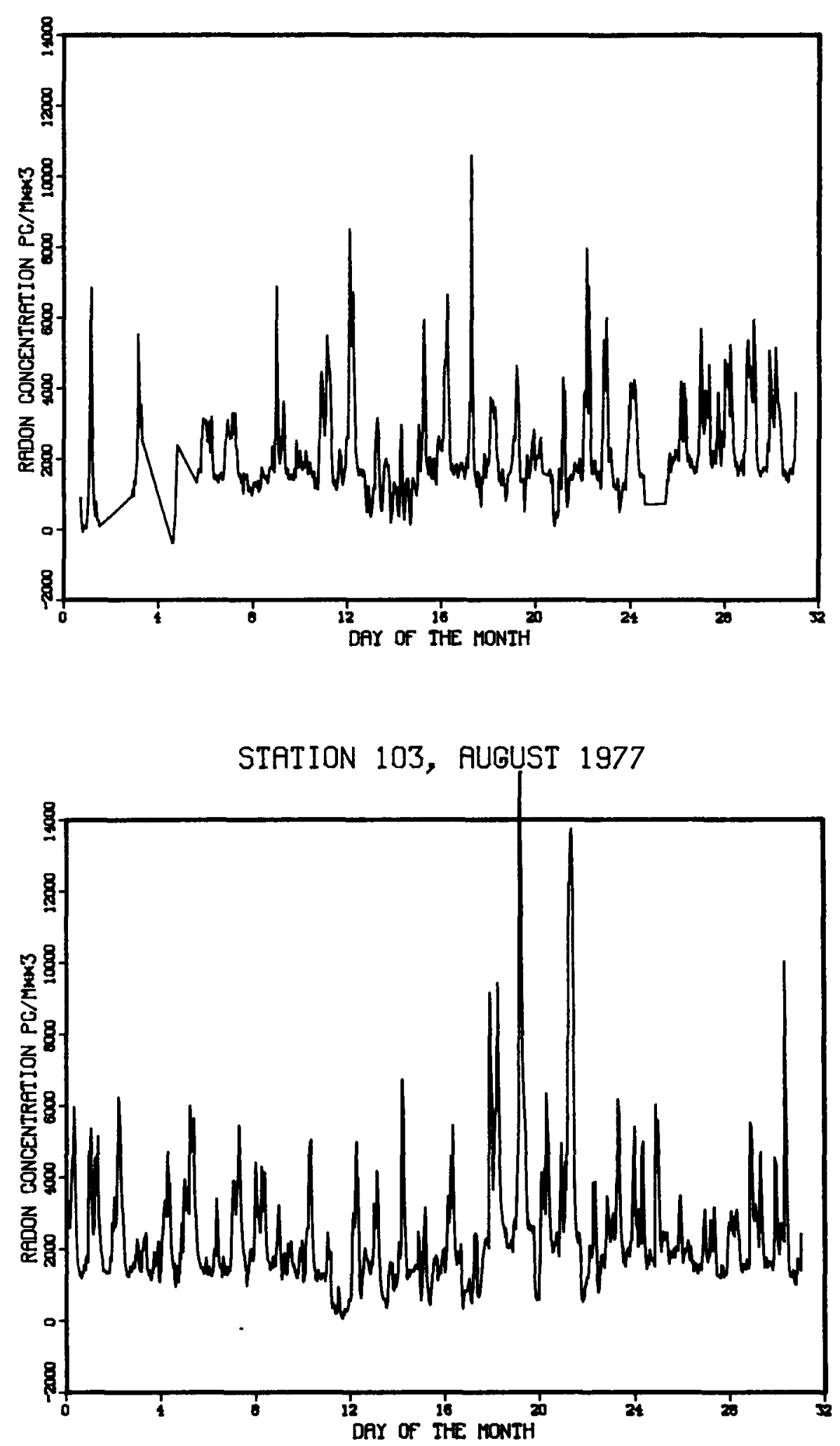

Figure A6. Radon Concentration by Hour at Station 103. 
STATION 103, SEPTEMBER 1977

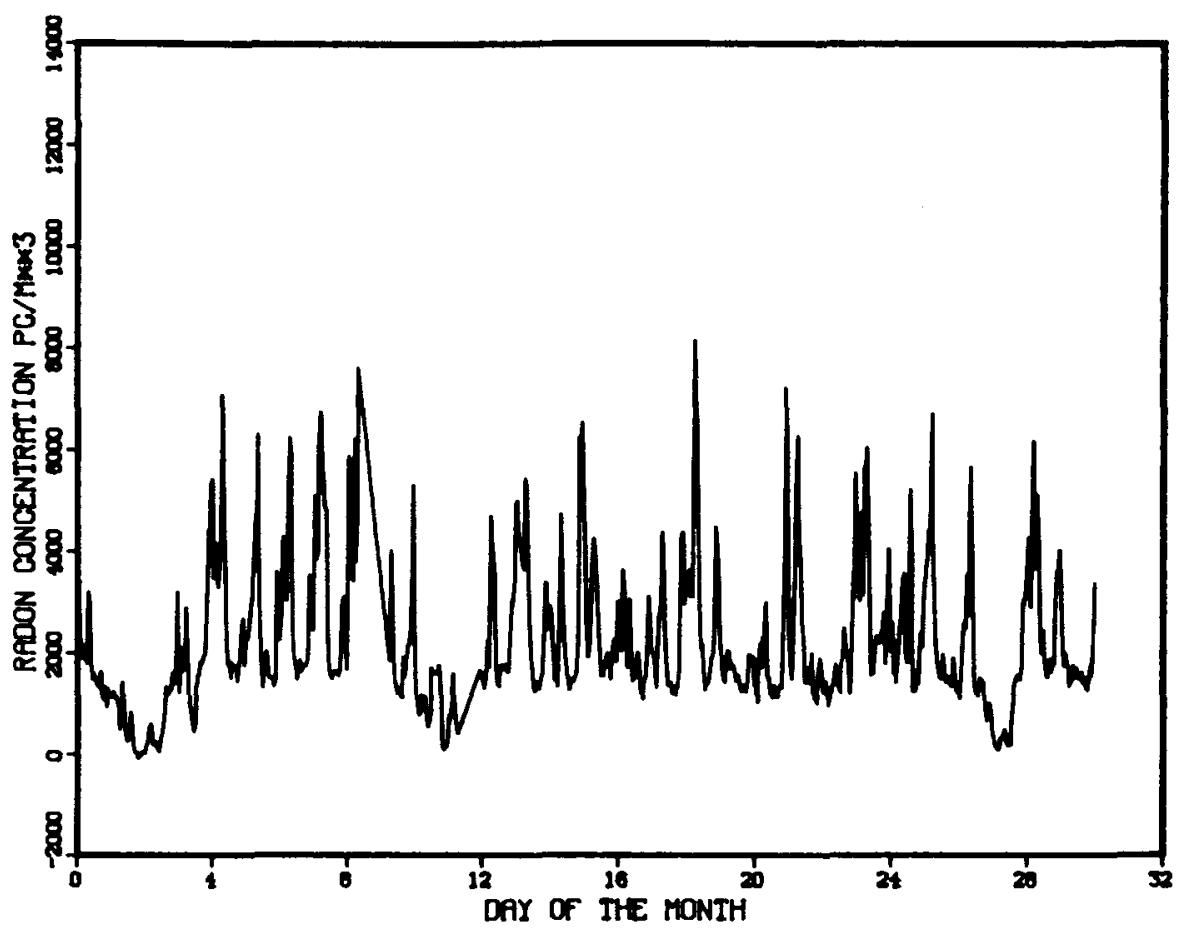

STATION 103, OCTOBER 1977

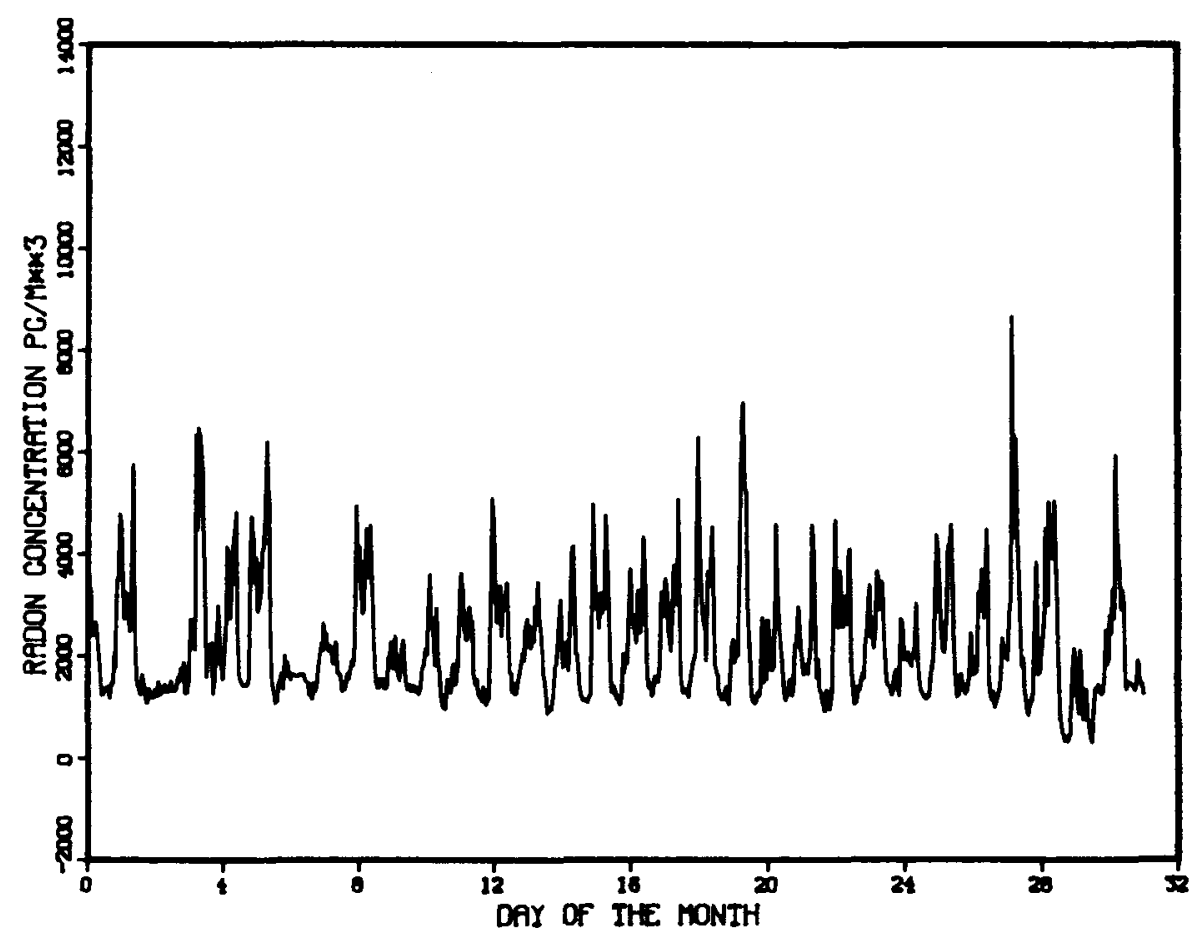

Figure A6. Continued. 
STATION 103, NOVEMBER 1977
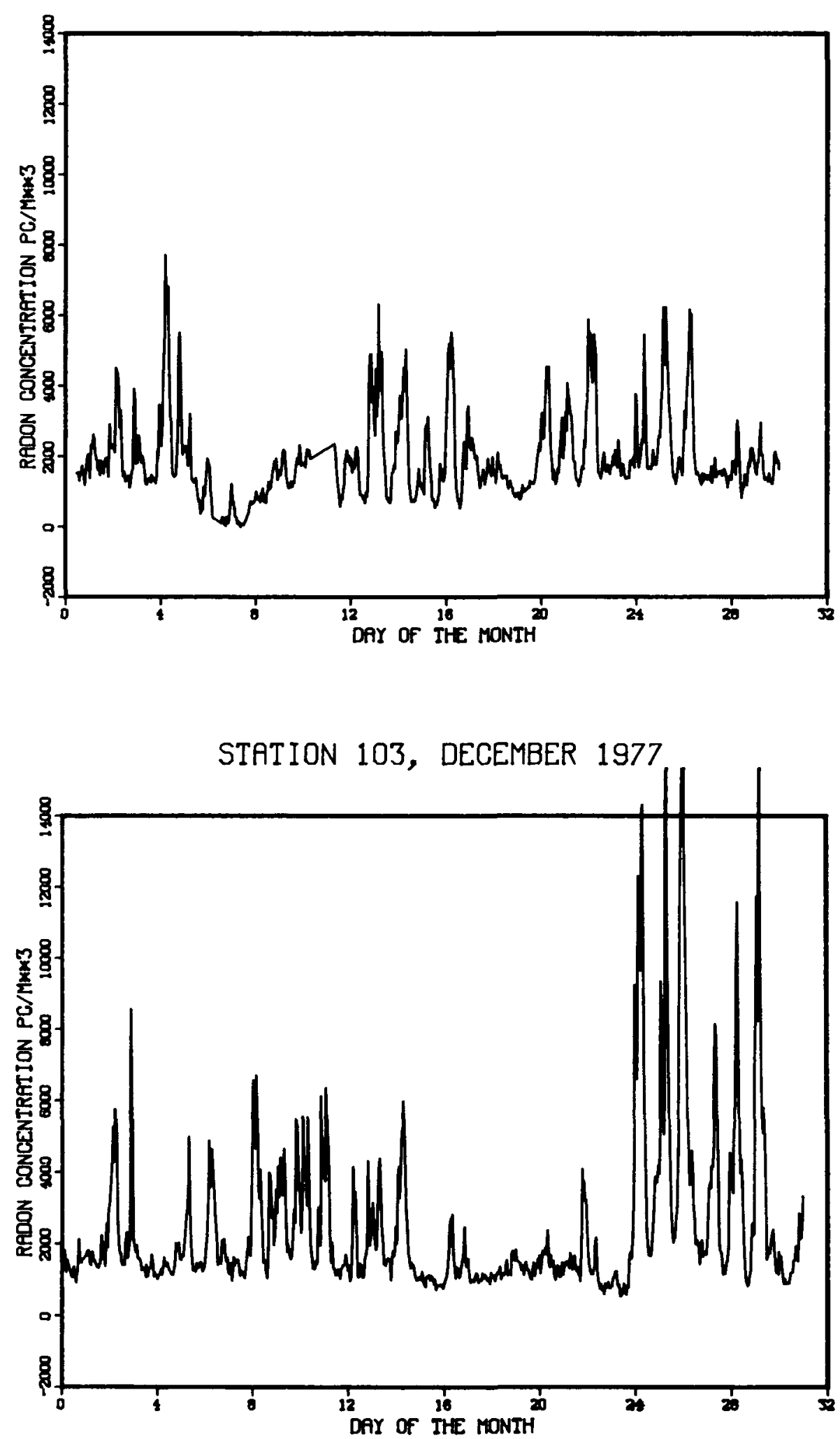

Figure A6. Continued. 
STATION 103, MRRCH 1978

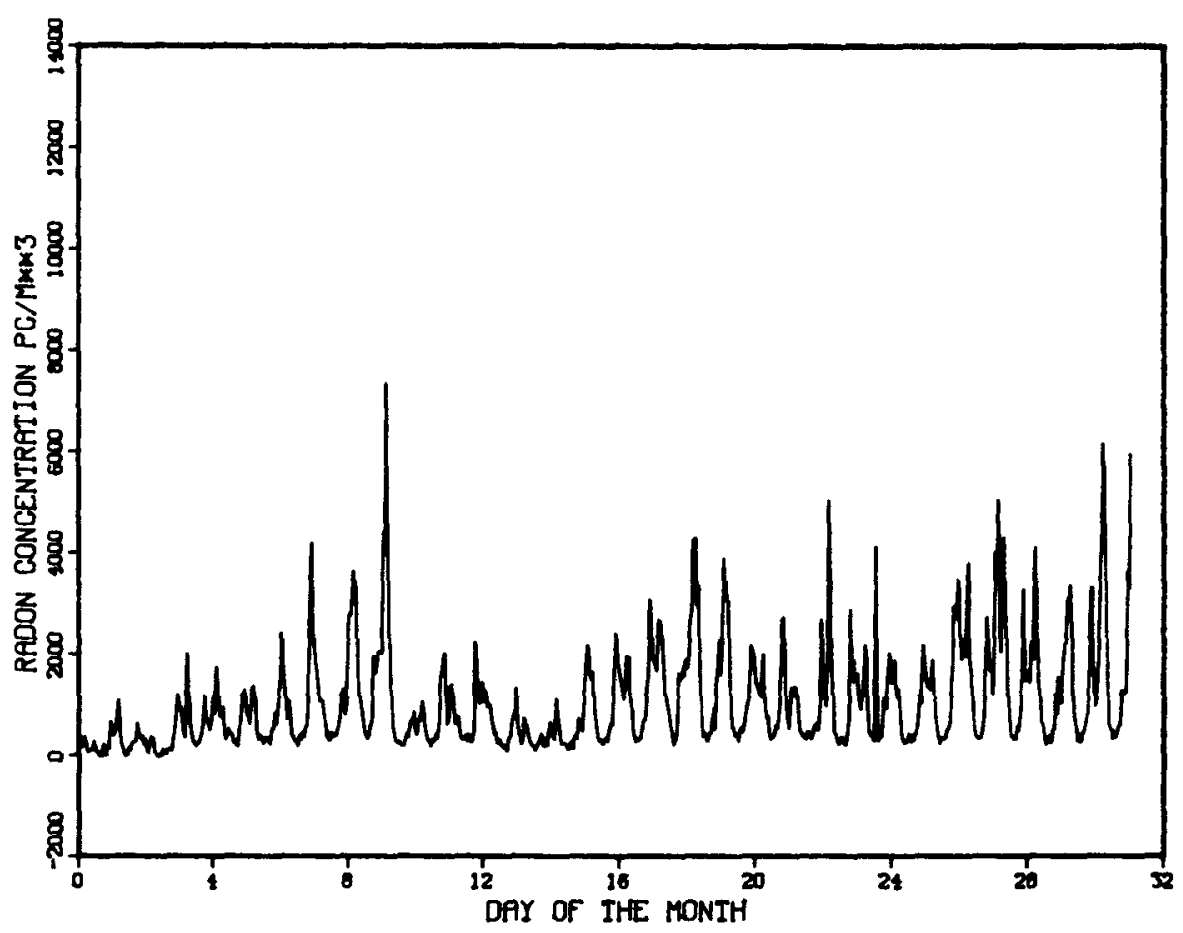

STATION 103, APRIL 1978

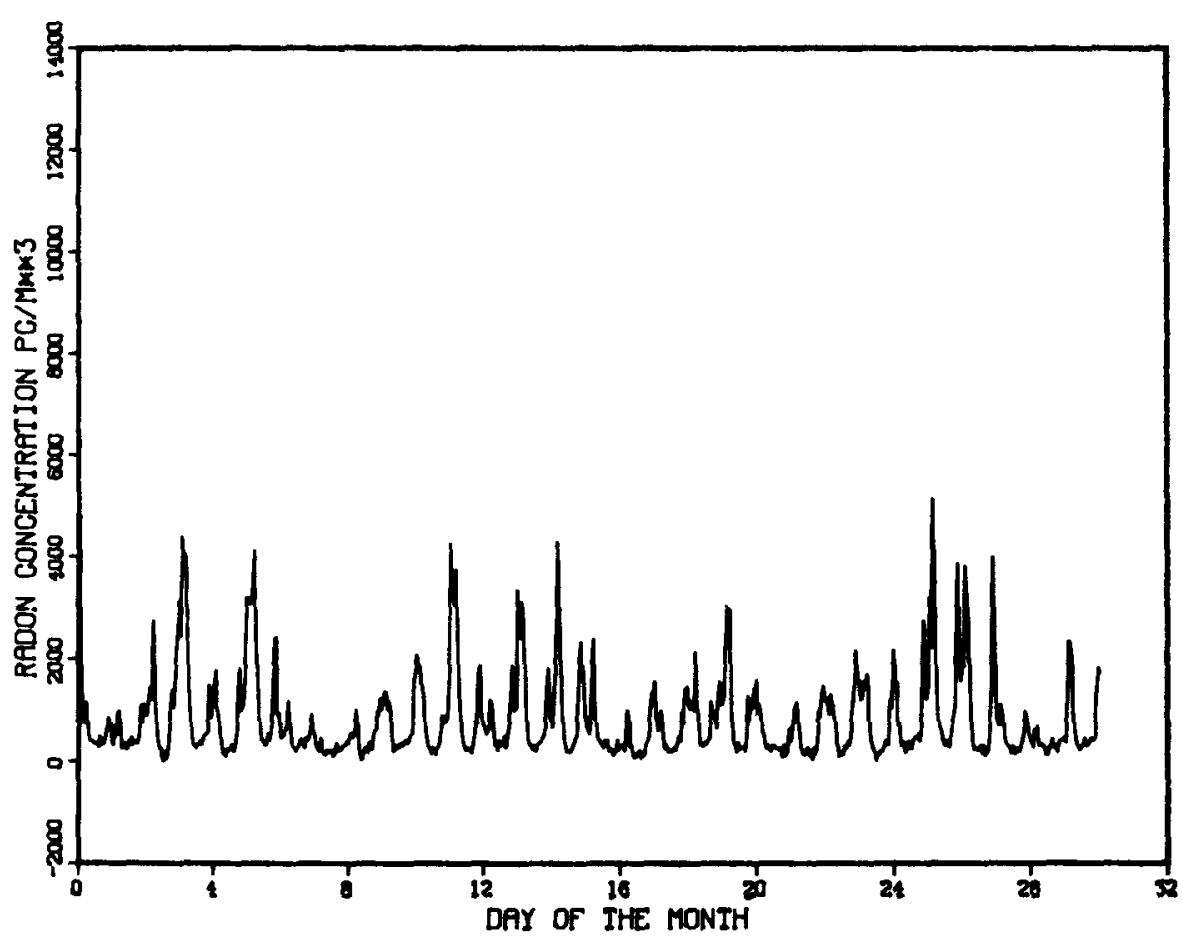

Figure A6. Continued. 


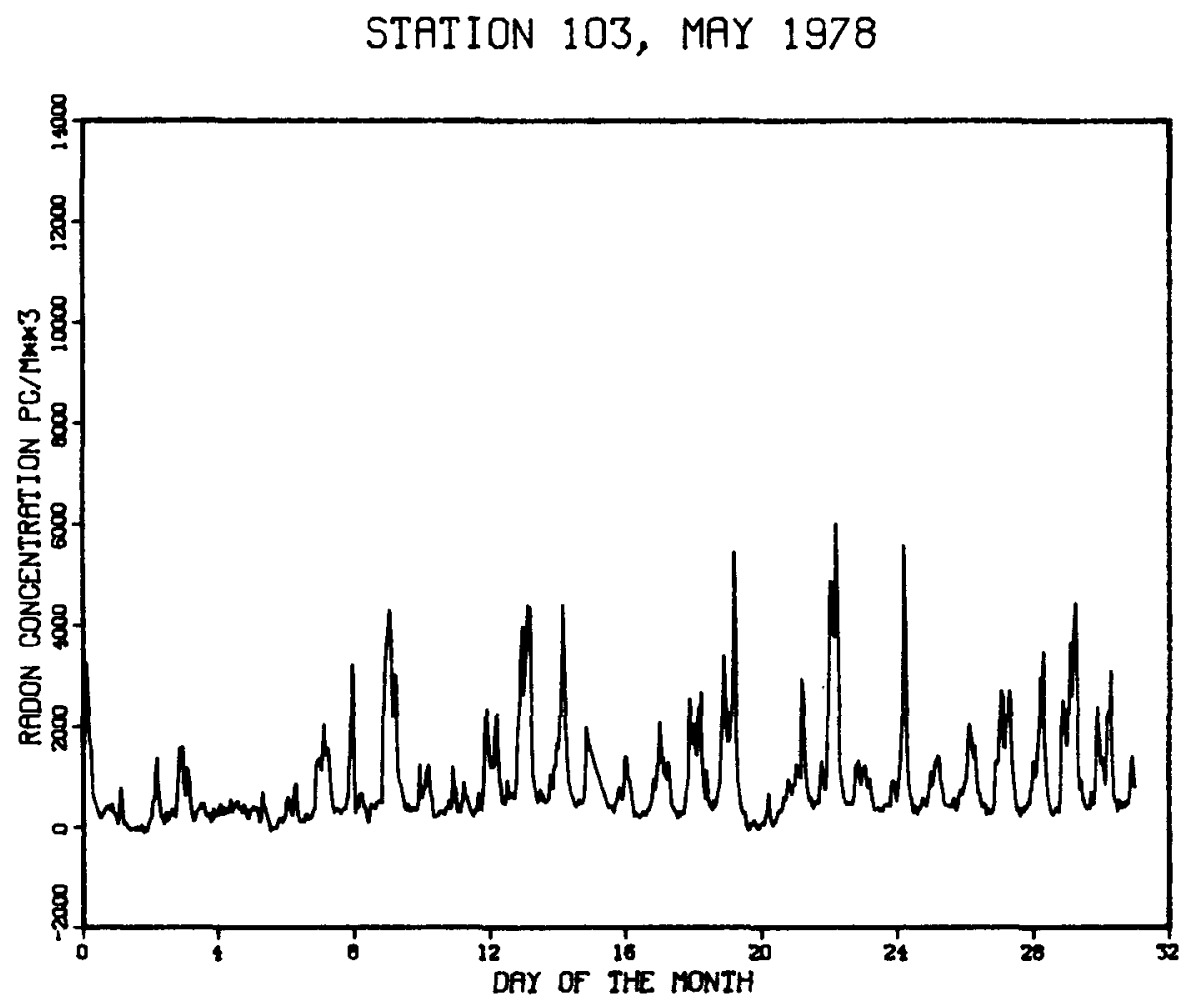

STATION 103, JUNE 1978

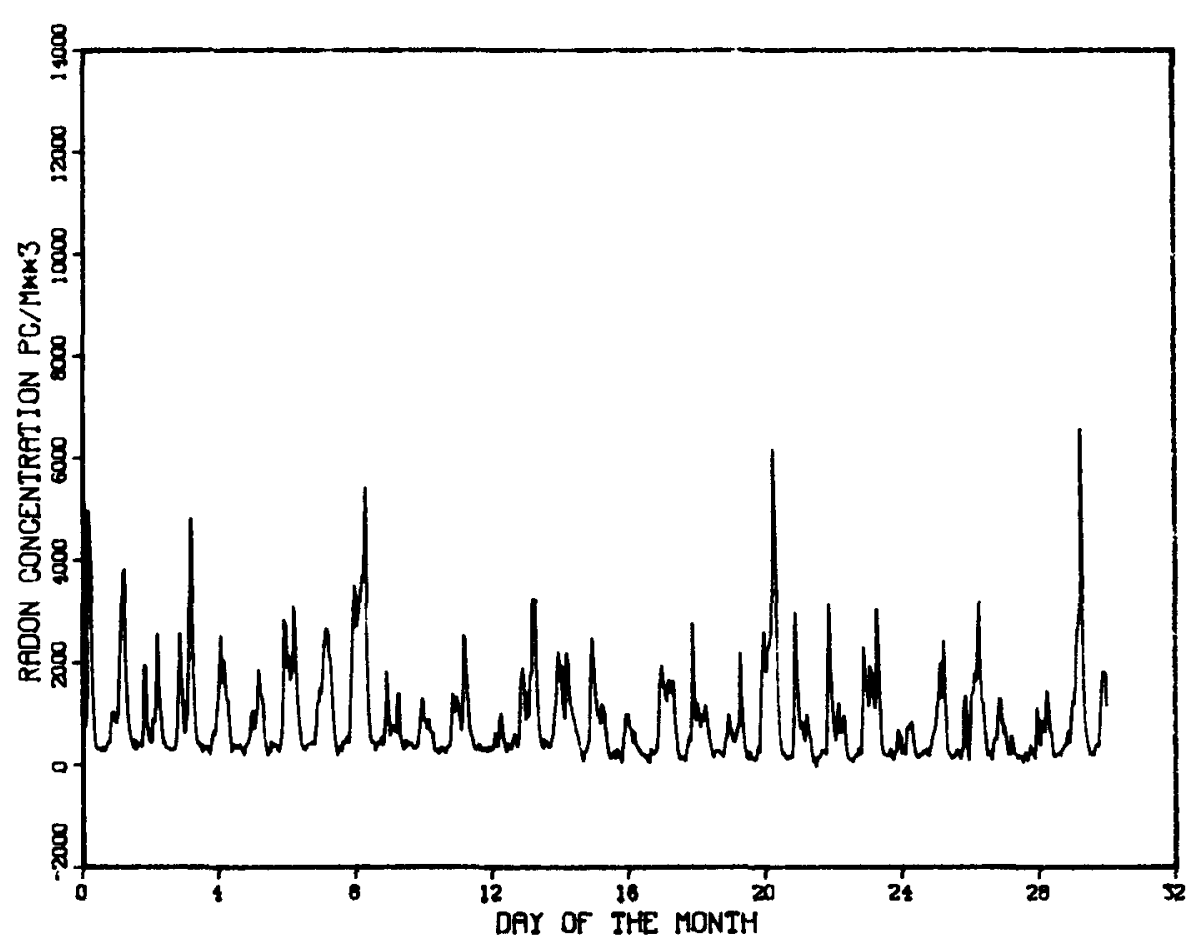

Figure A6. Continued. 
STATION 103, JULY 1978

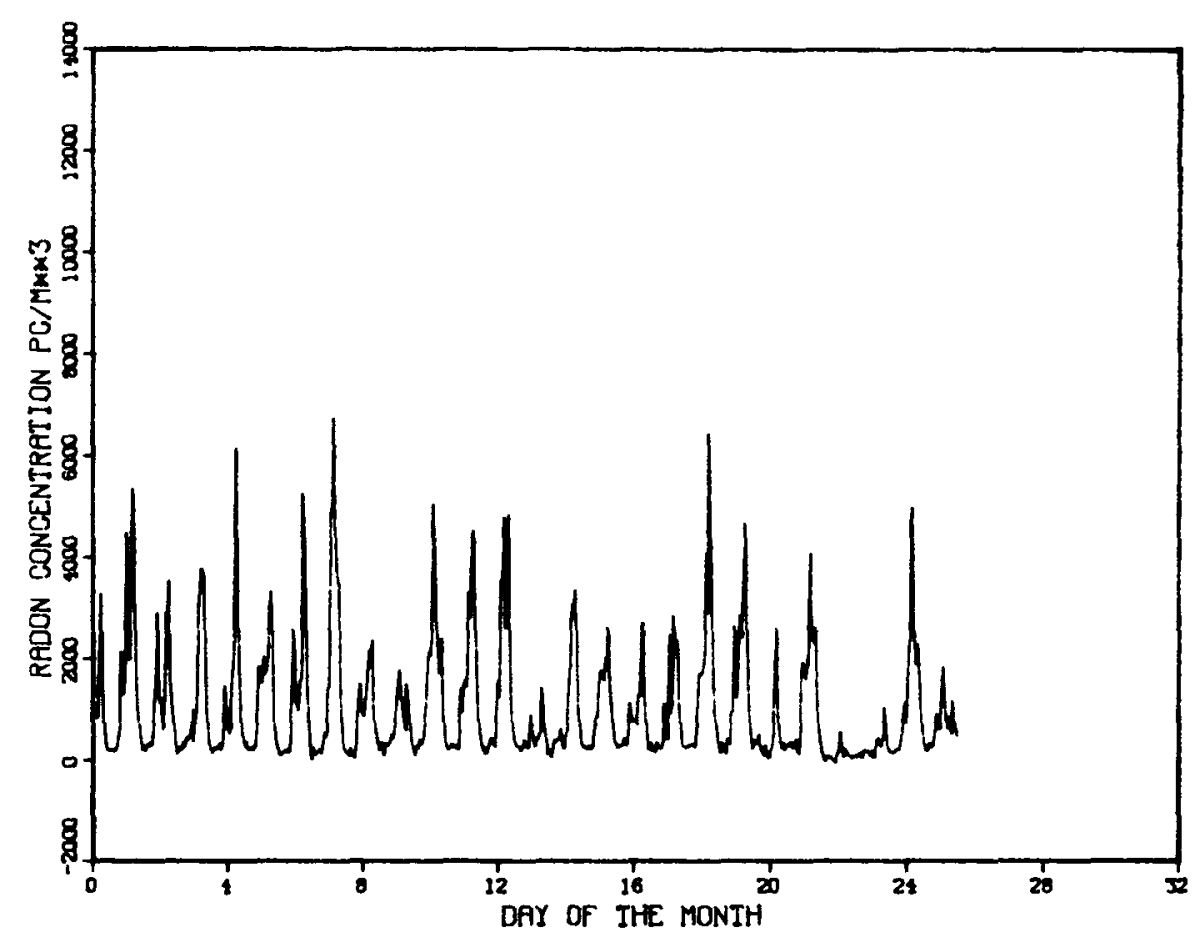

Figure A6. Continued. 
STATION 104, JULY 1977

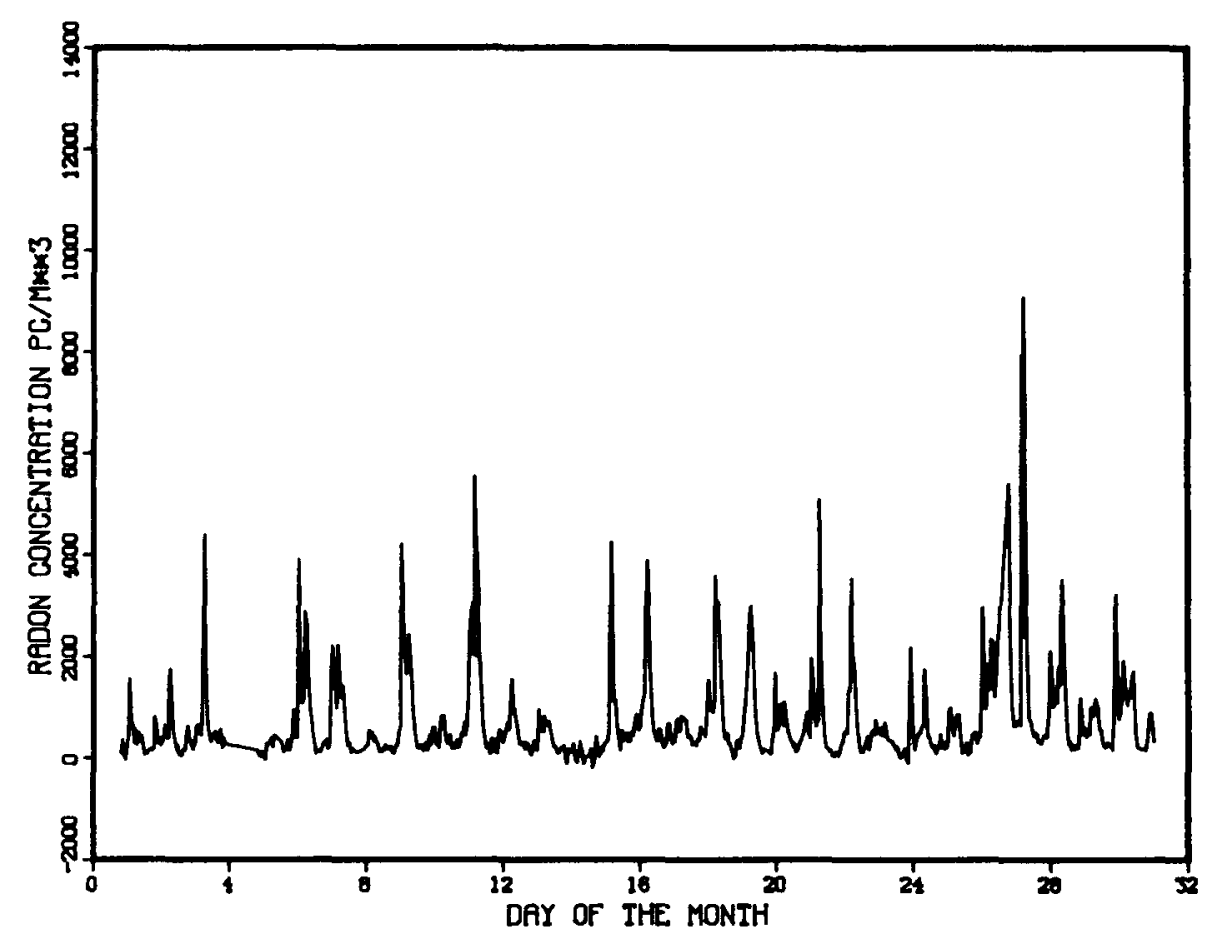

STATION 104, AUGUST 1977

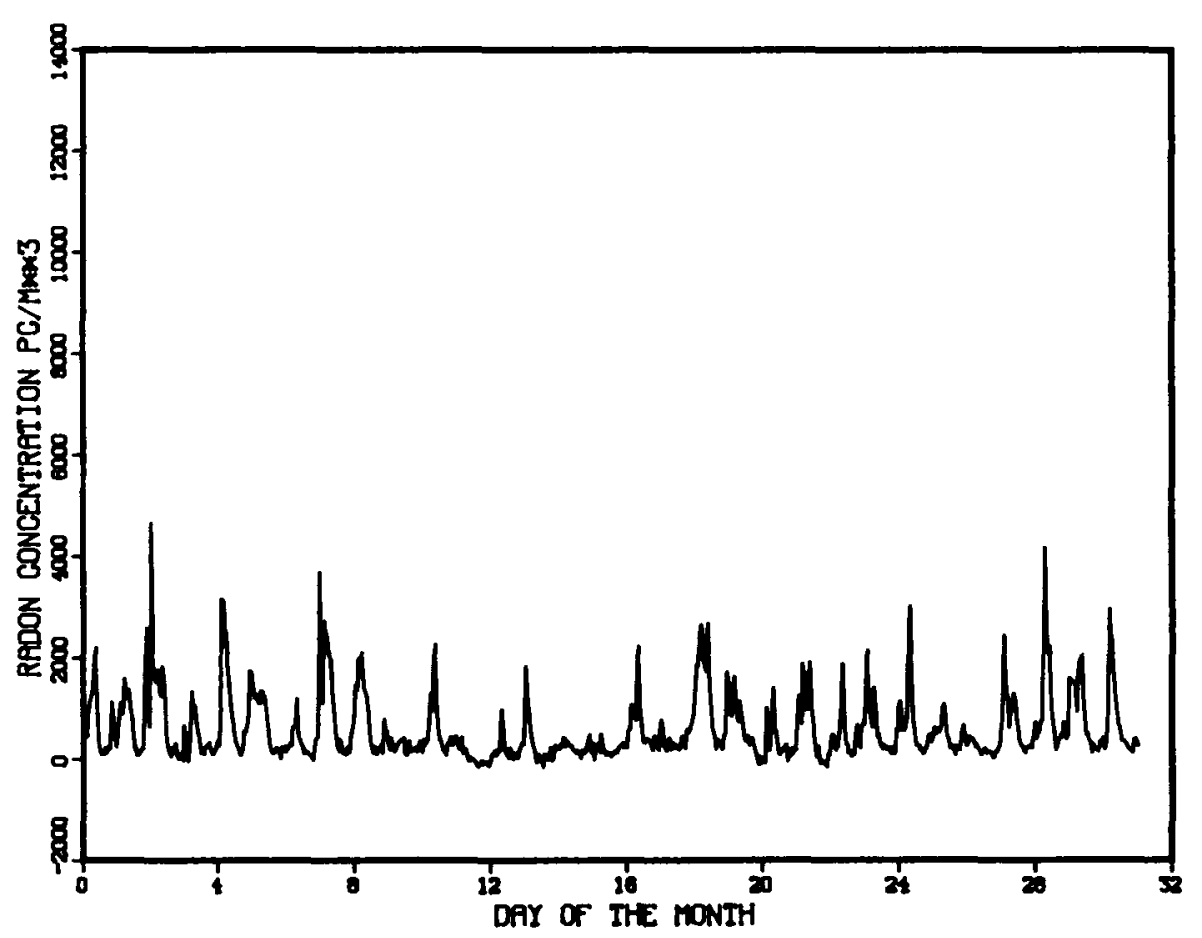

Figure A7. Radon Concentration by Hour at Station 104. 


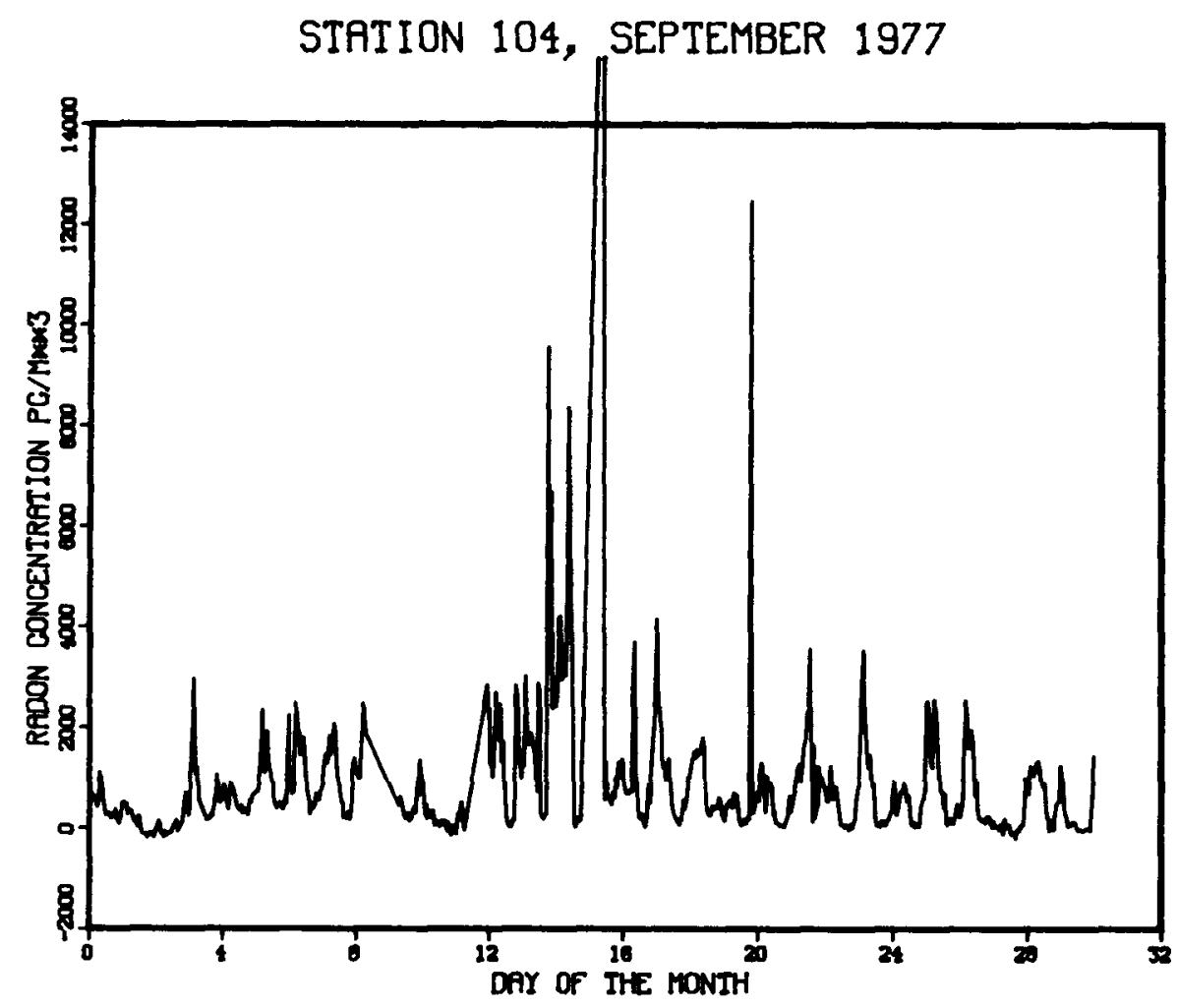

STATION 104, OCTOBER 1977

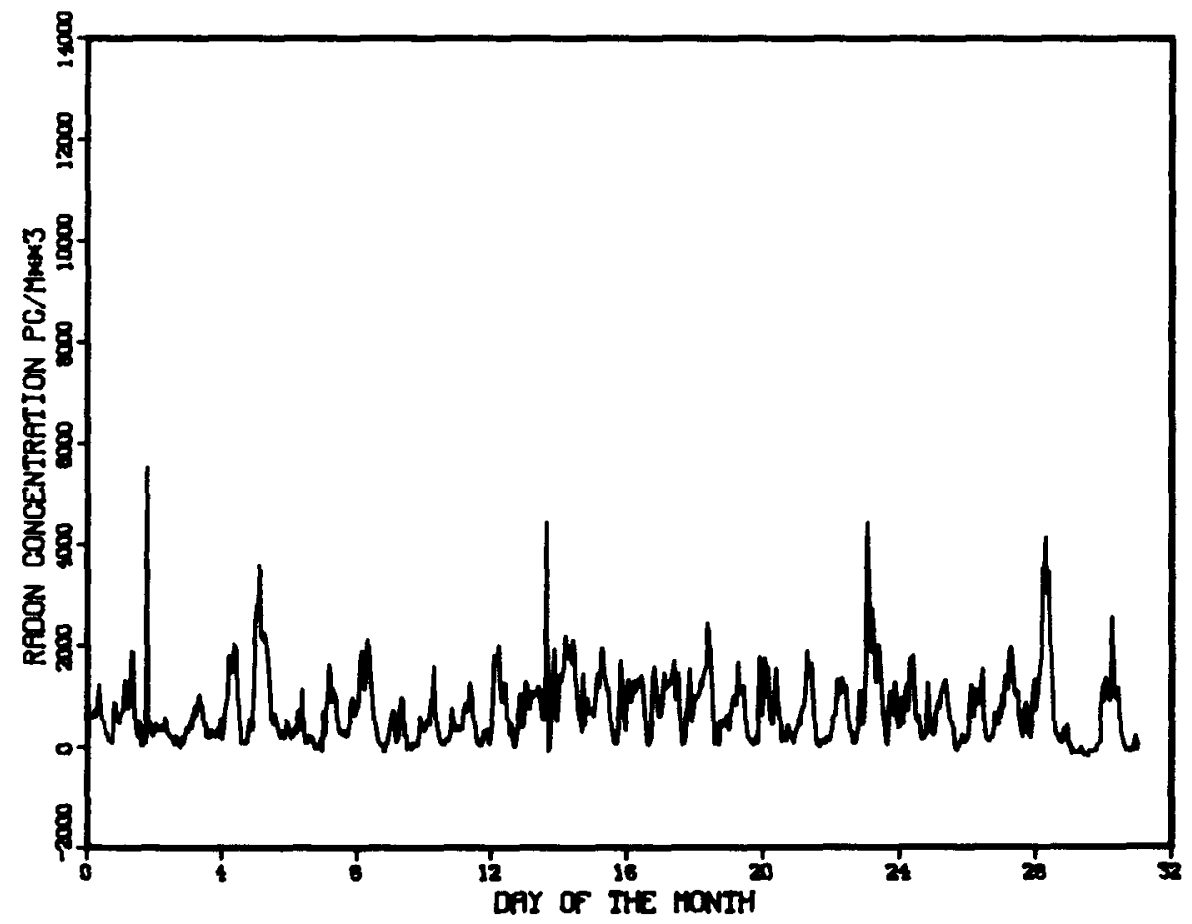

Figure A7. Continued. 
STATION 104, NOVEMBER 1977

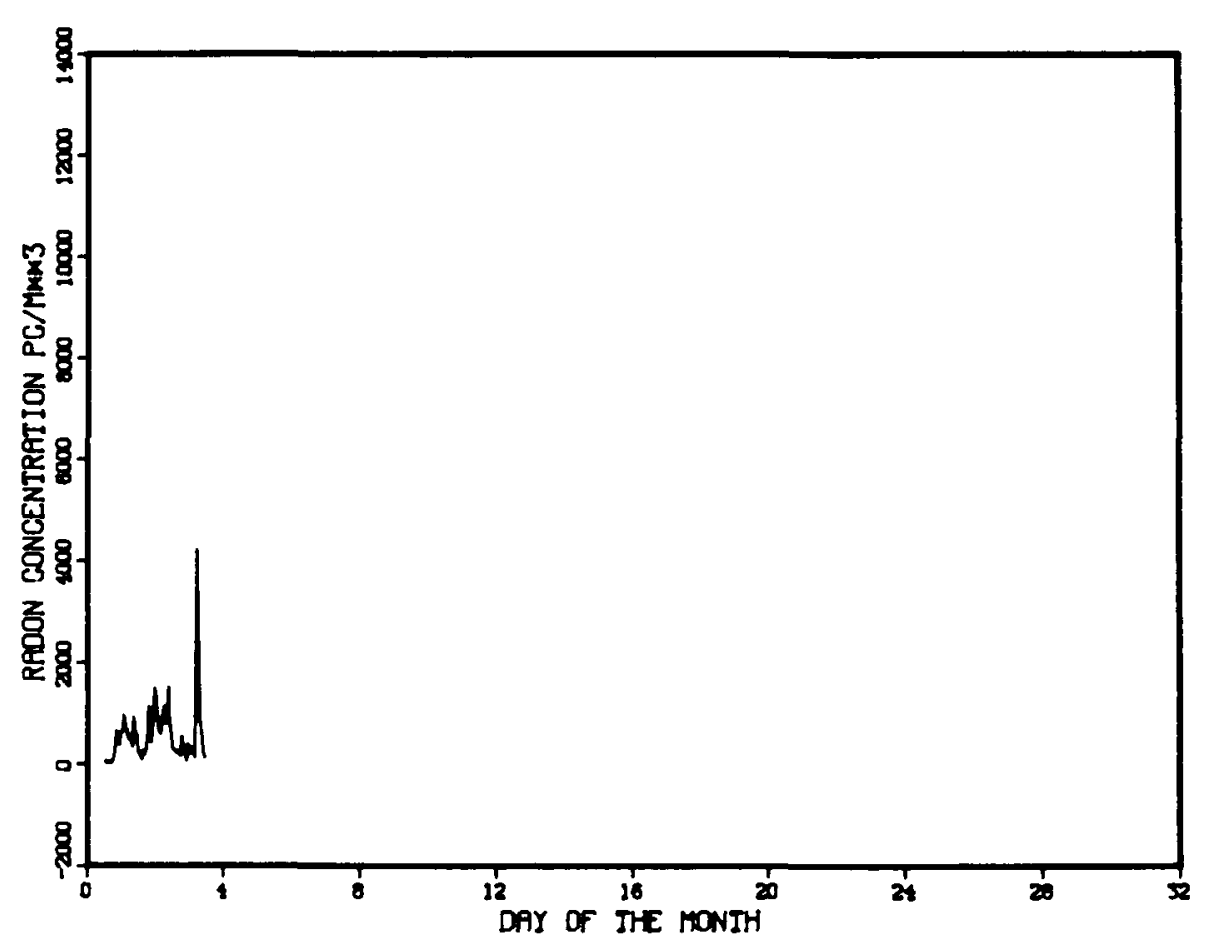

STATION 104, DECEMBER 1977

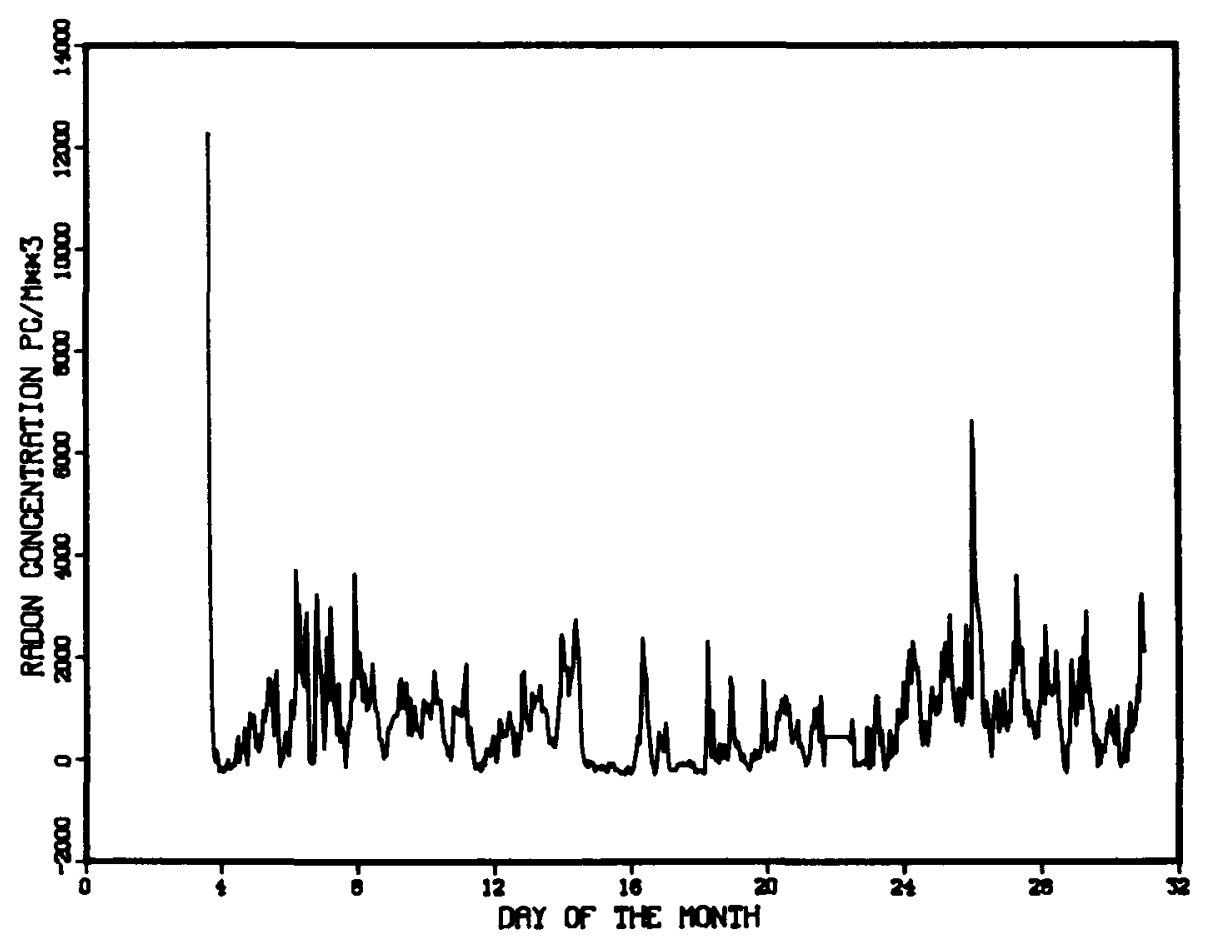

Figure A7. Continued. 


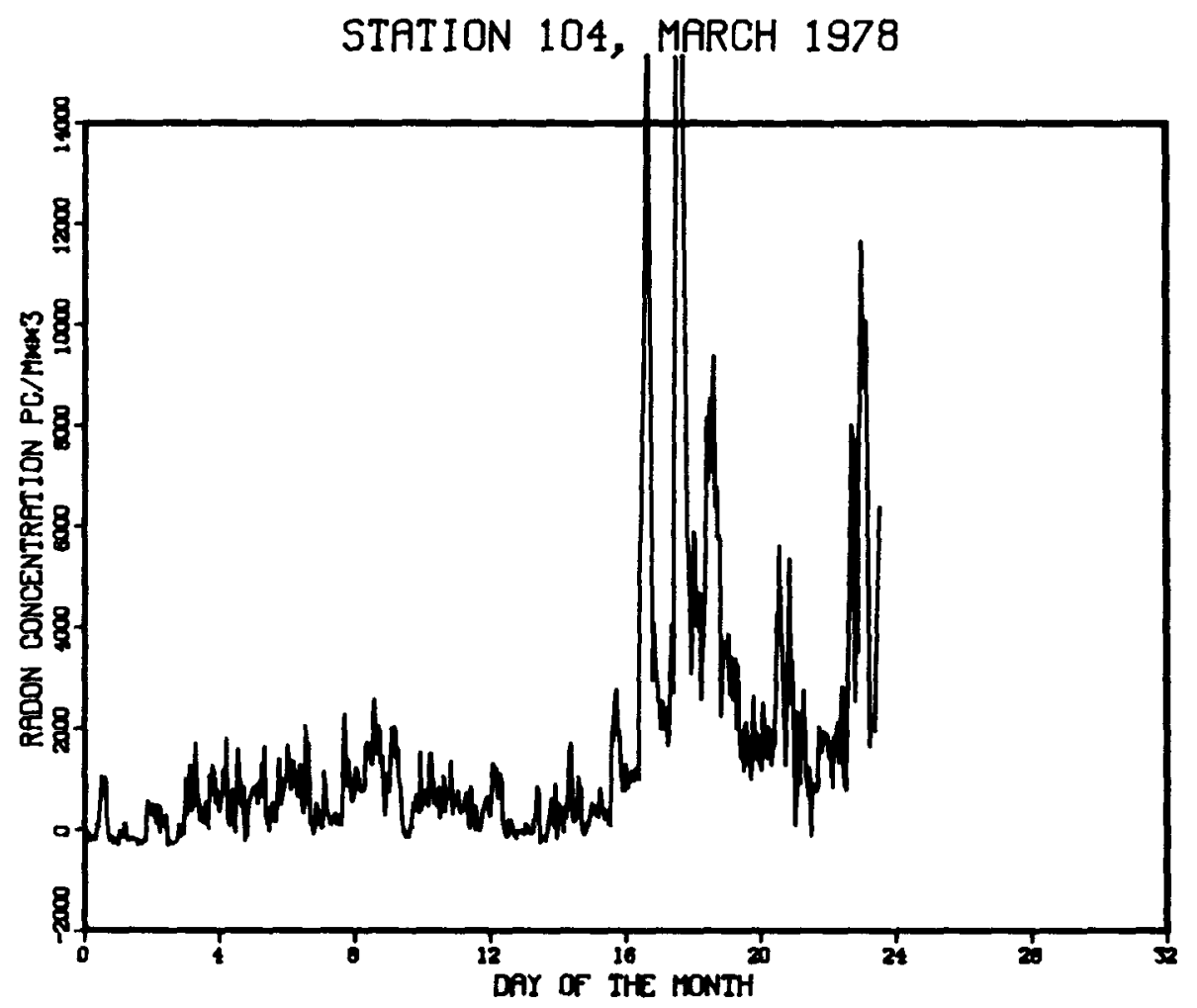

Figure A7. Continued. 
STATION 102, JULY 1977

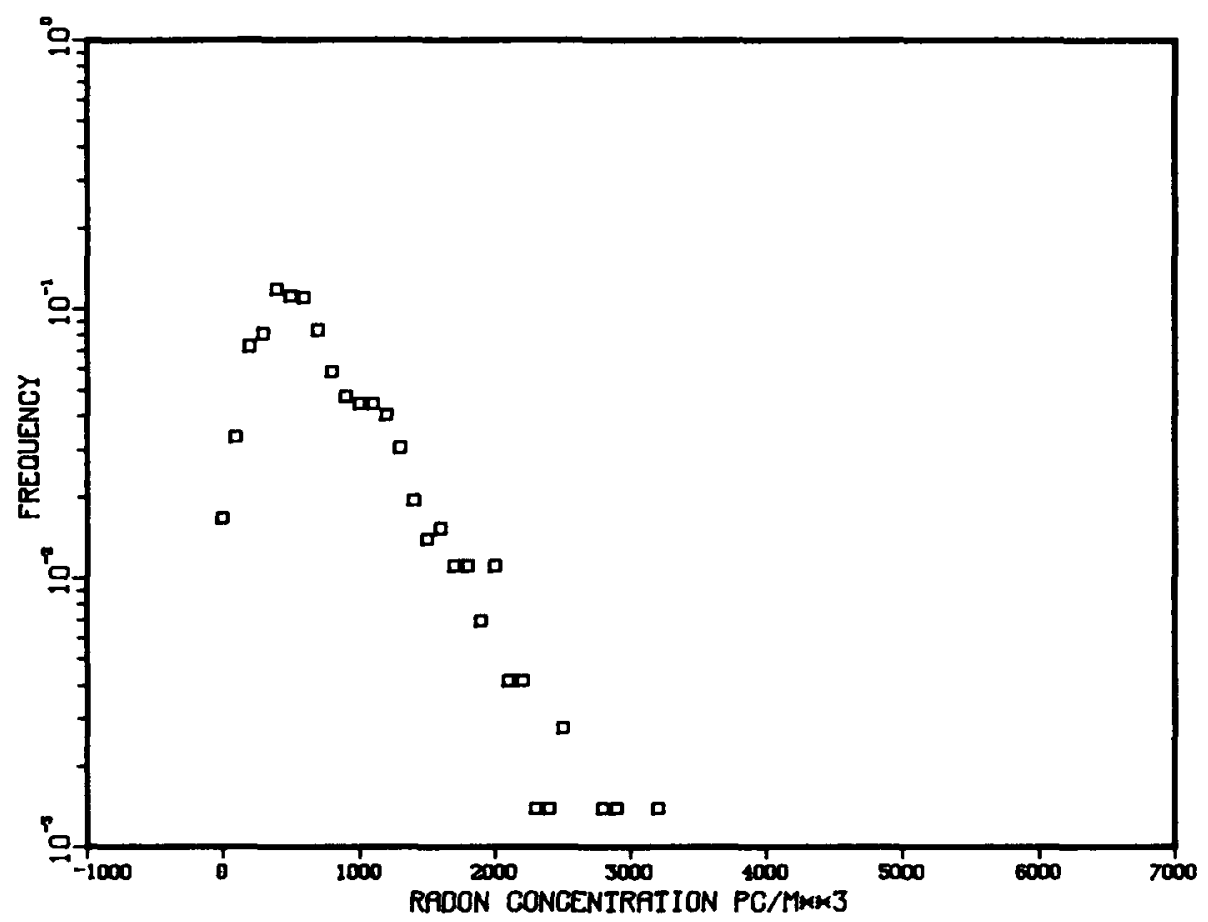

STATION 102, RUGUST 1977

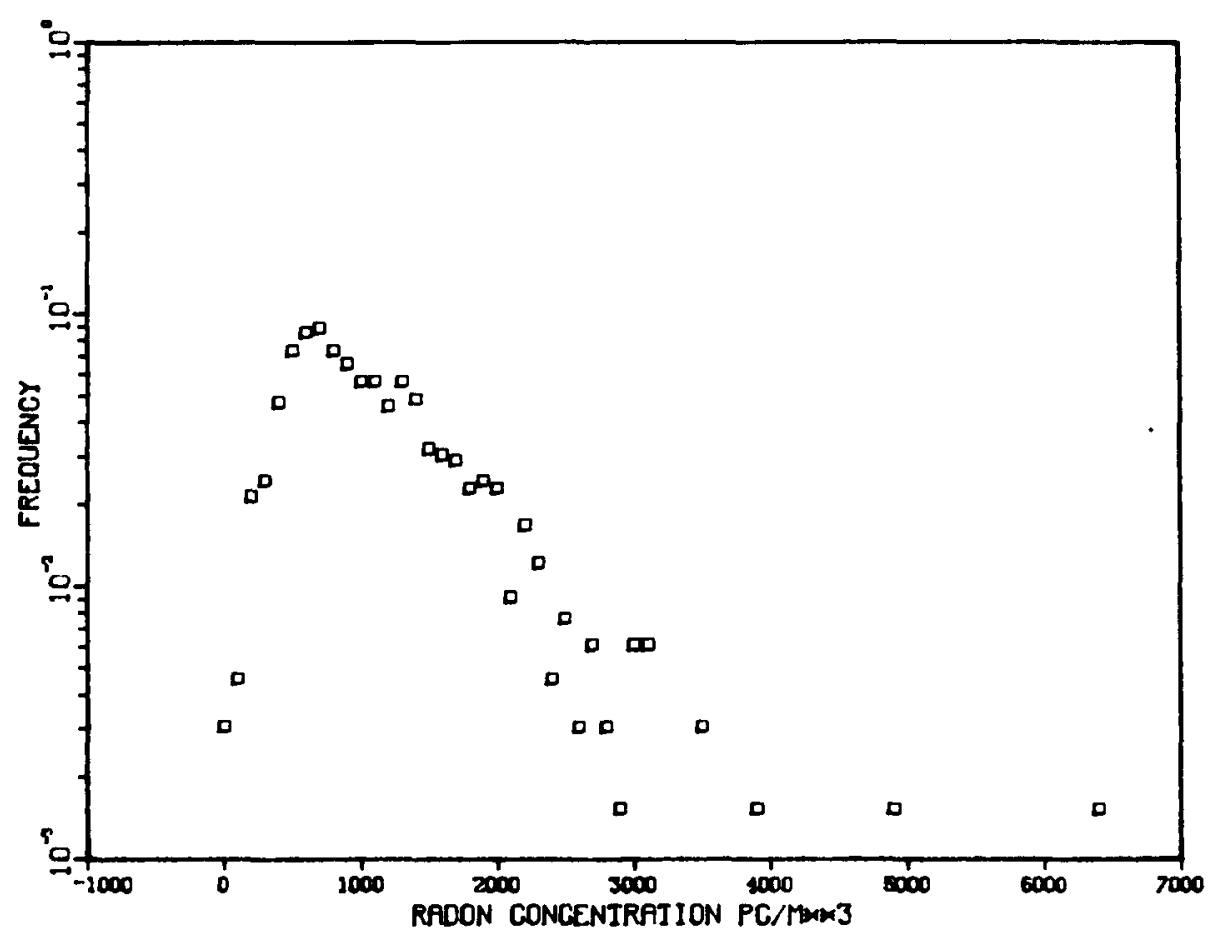

Figure A8. Radon-Concentration Frequency at Station 102. 
STATION 102, SEPTEMBER 1977

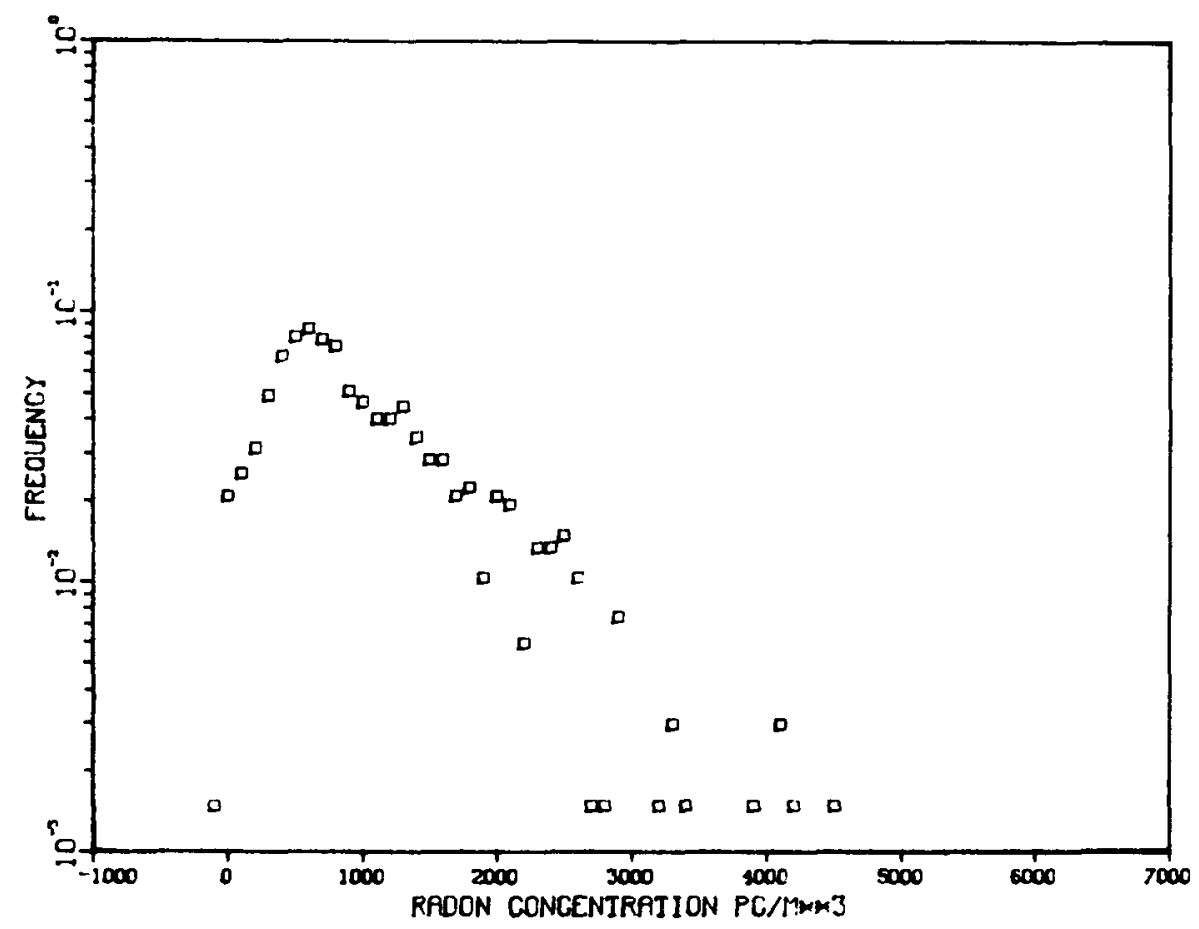

STATION 102, OCTOBER 1977

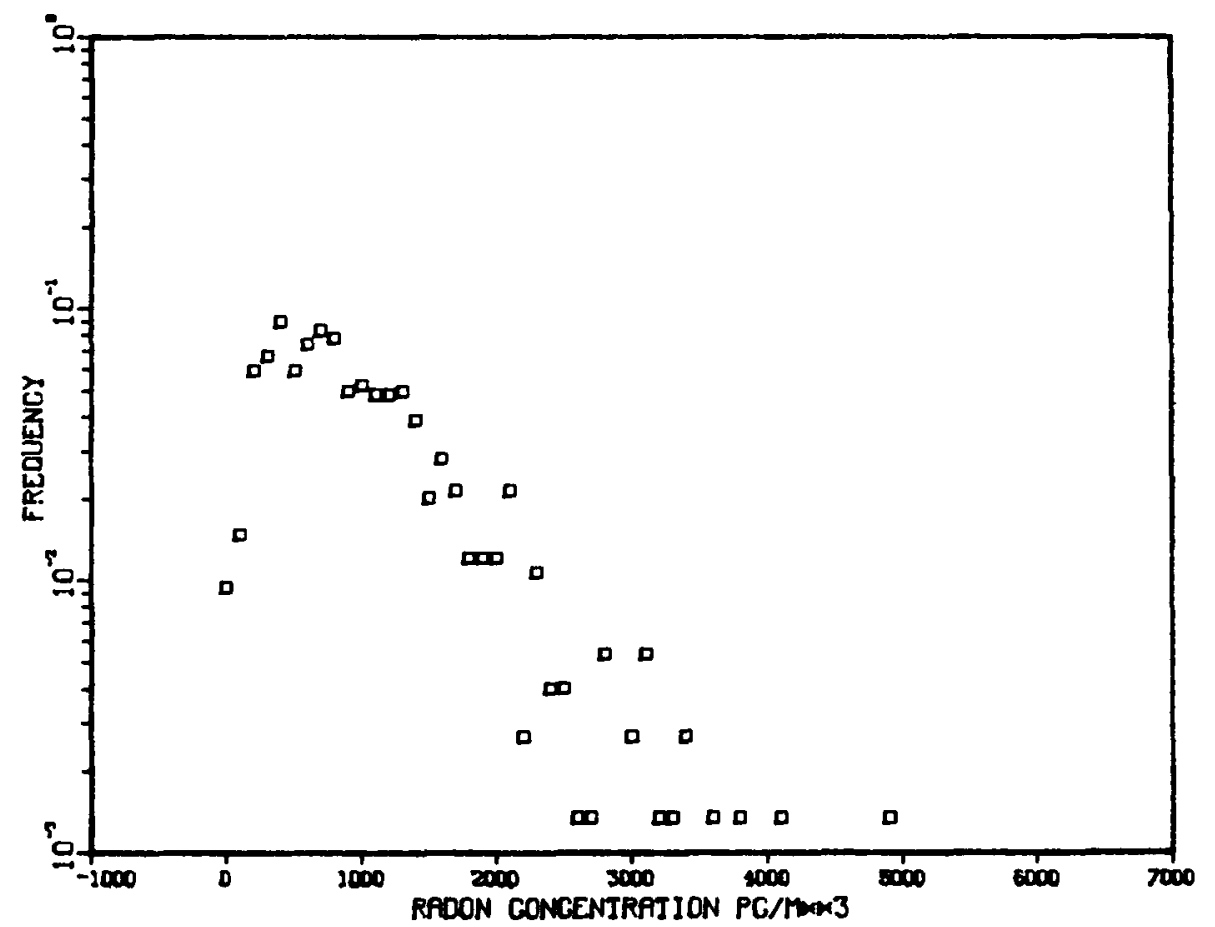

Figure A8. Continued. 
STATION 102, NOVEMBER 1977

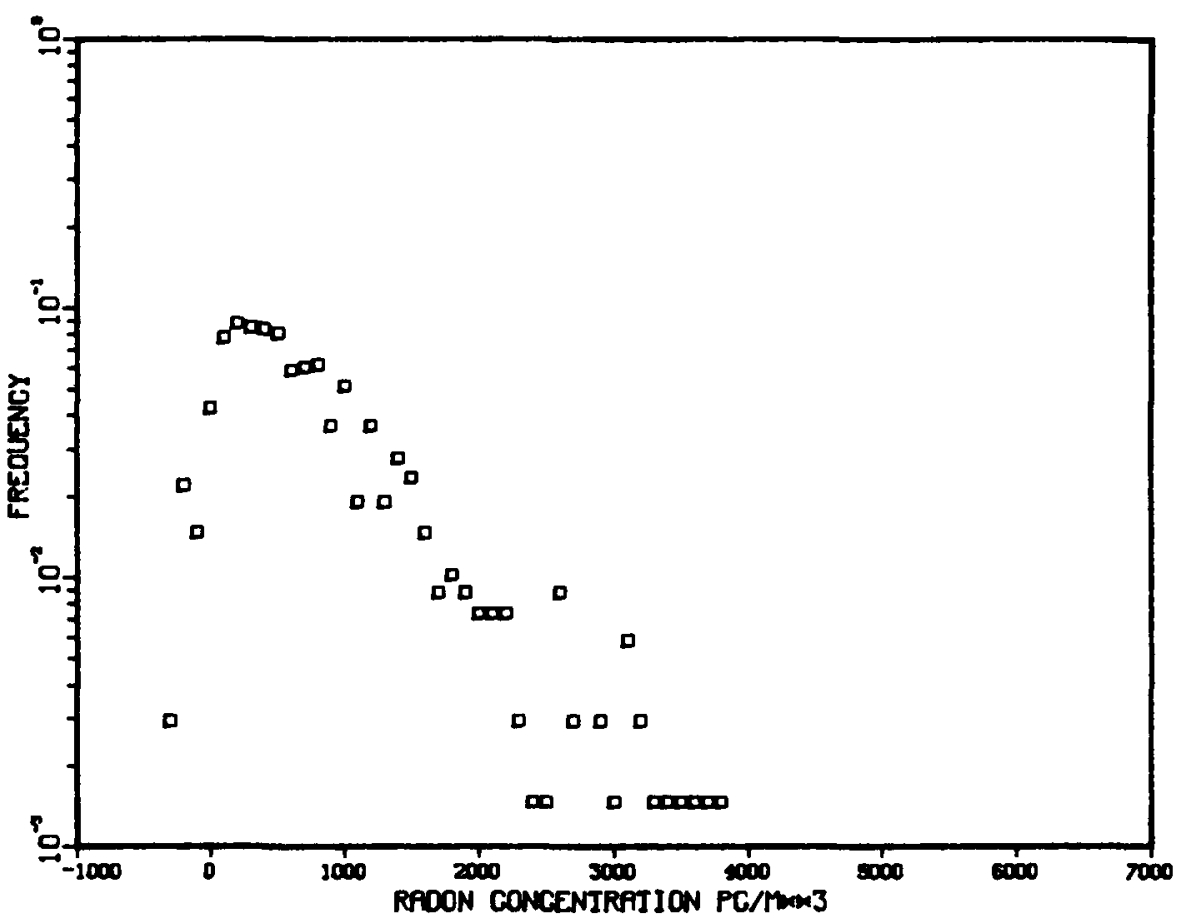

STATION 102, DECEMBER 1977

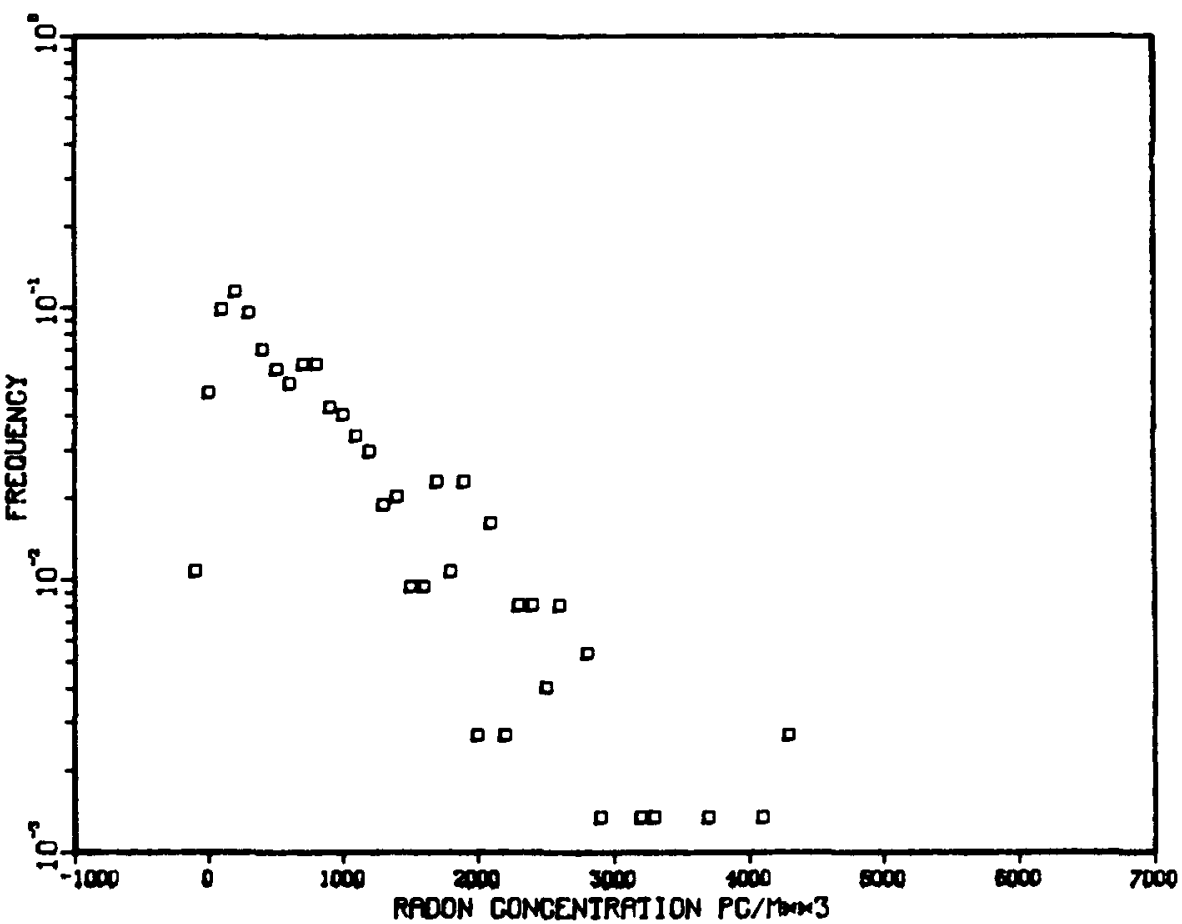

Figure A8. Continued. 
STATION 102, MARCH 1978

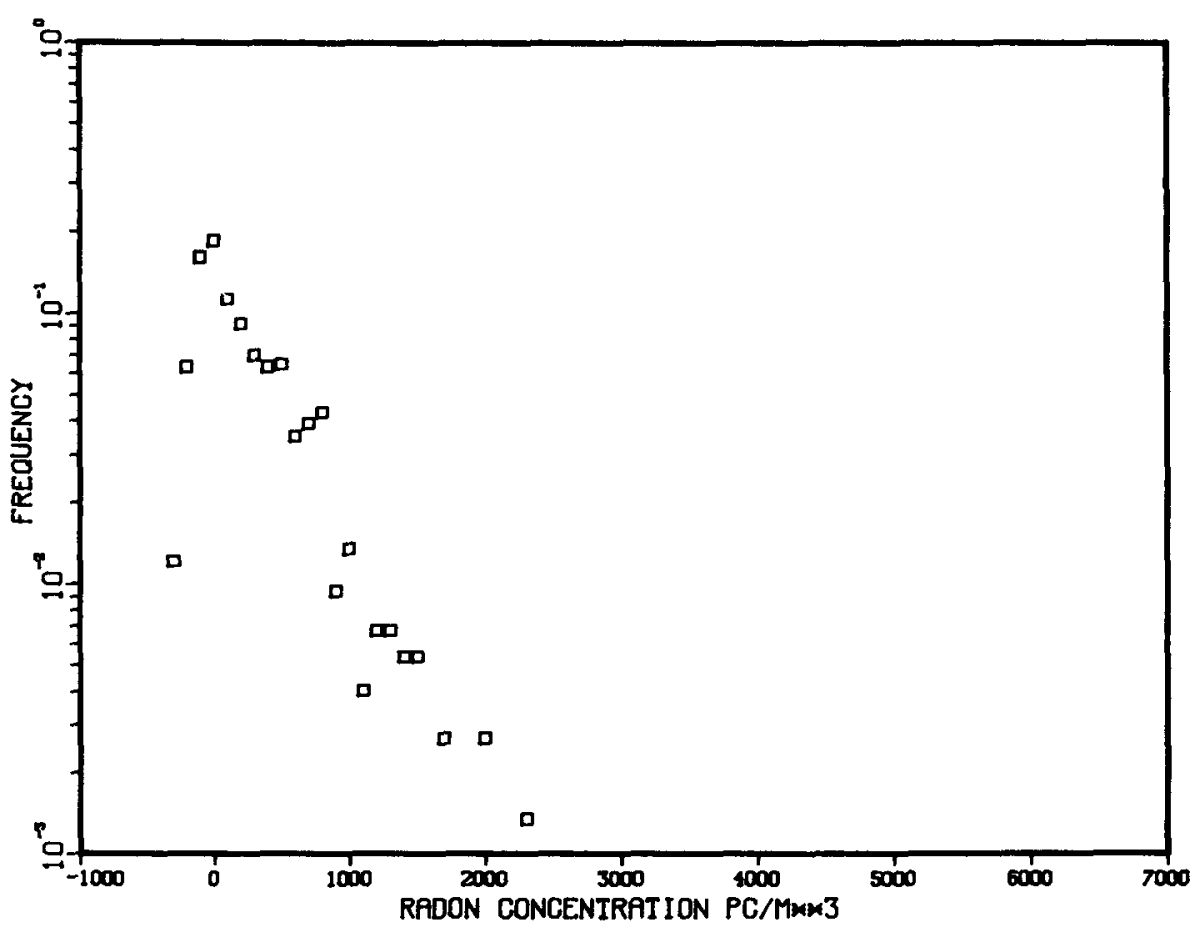

STATION 102, APRIL 1978

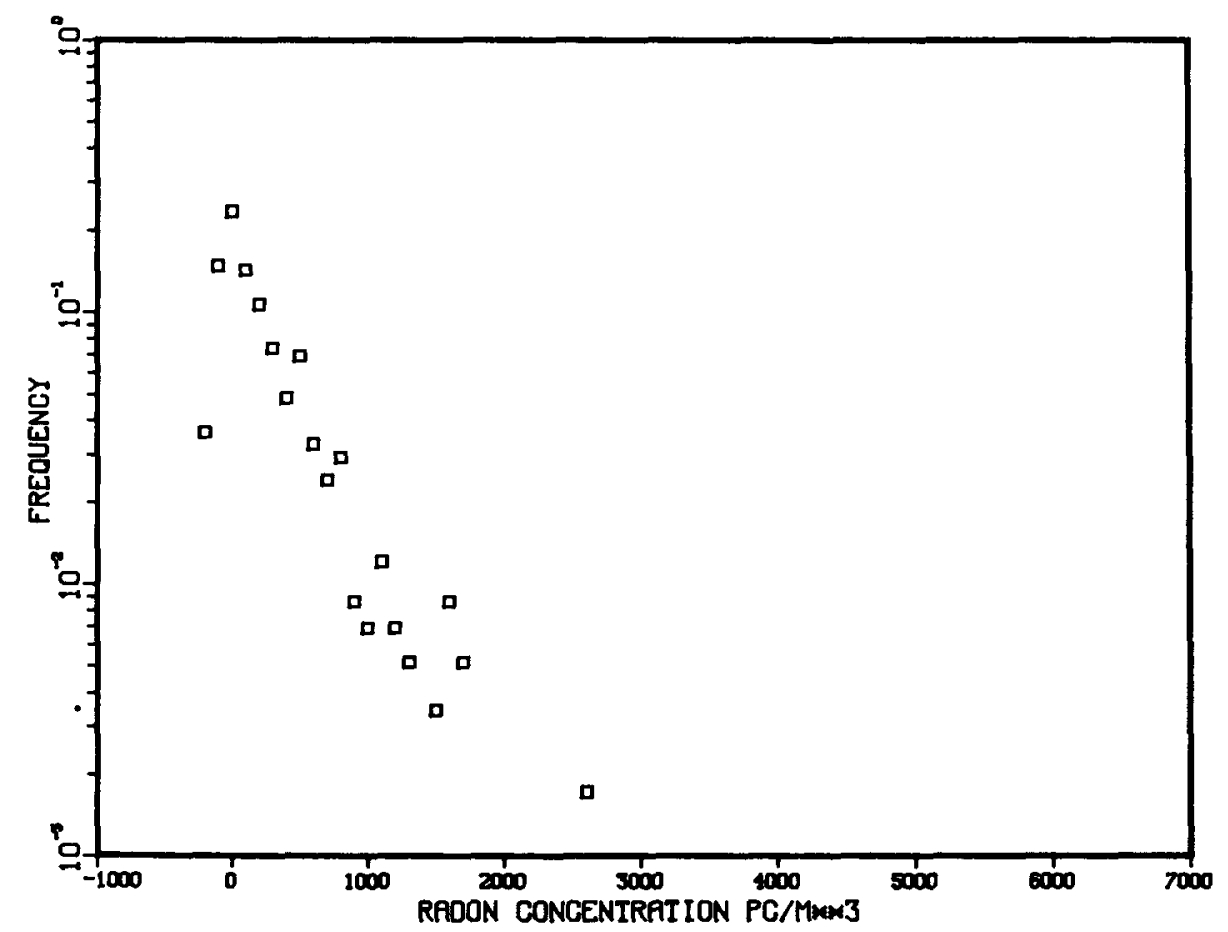

Figure A8. Continued. 
STATION 102, MAY 1978

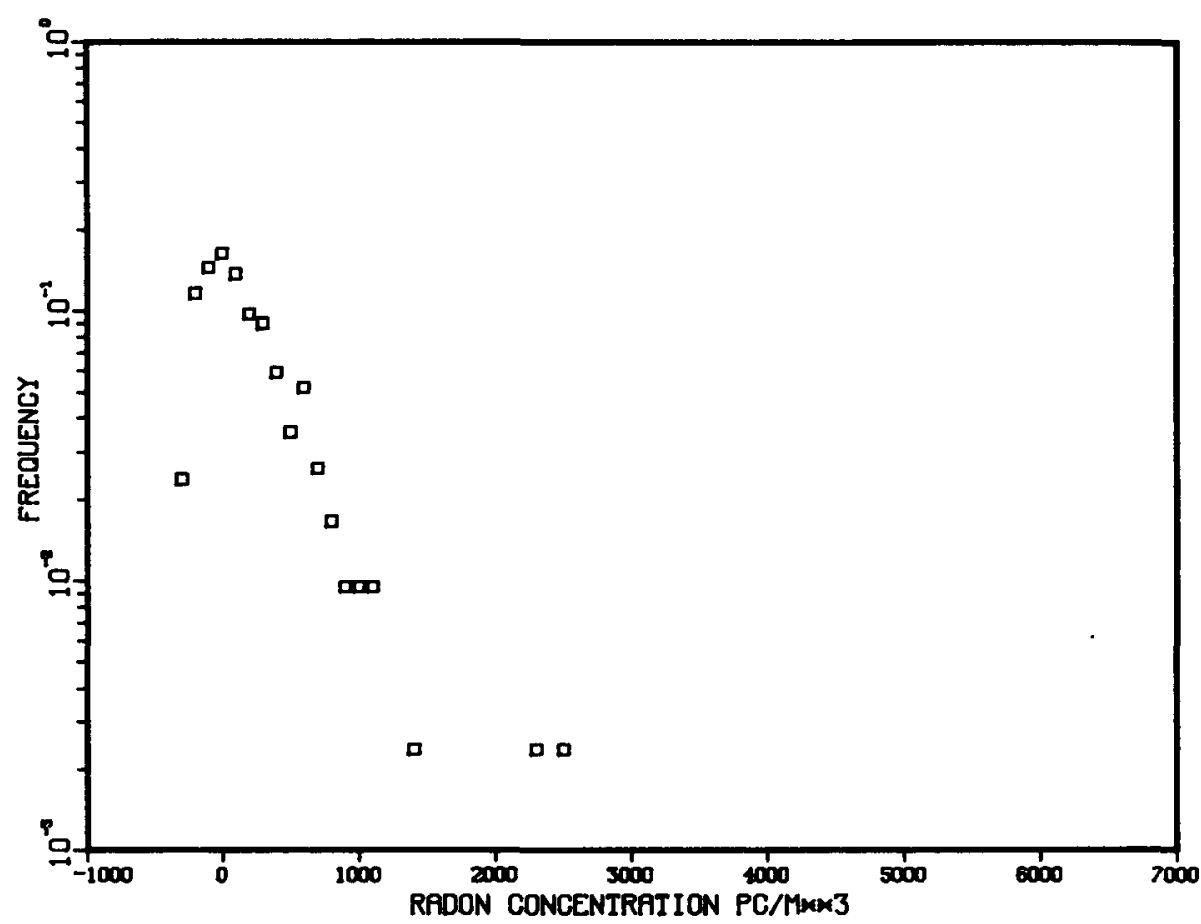

STATION 102, JUNE 1978

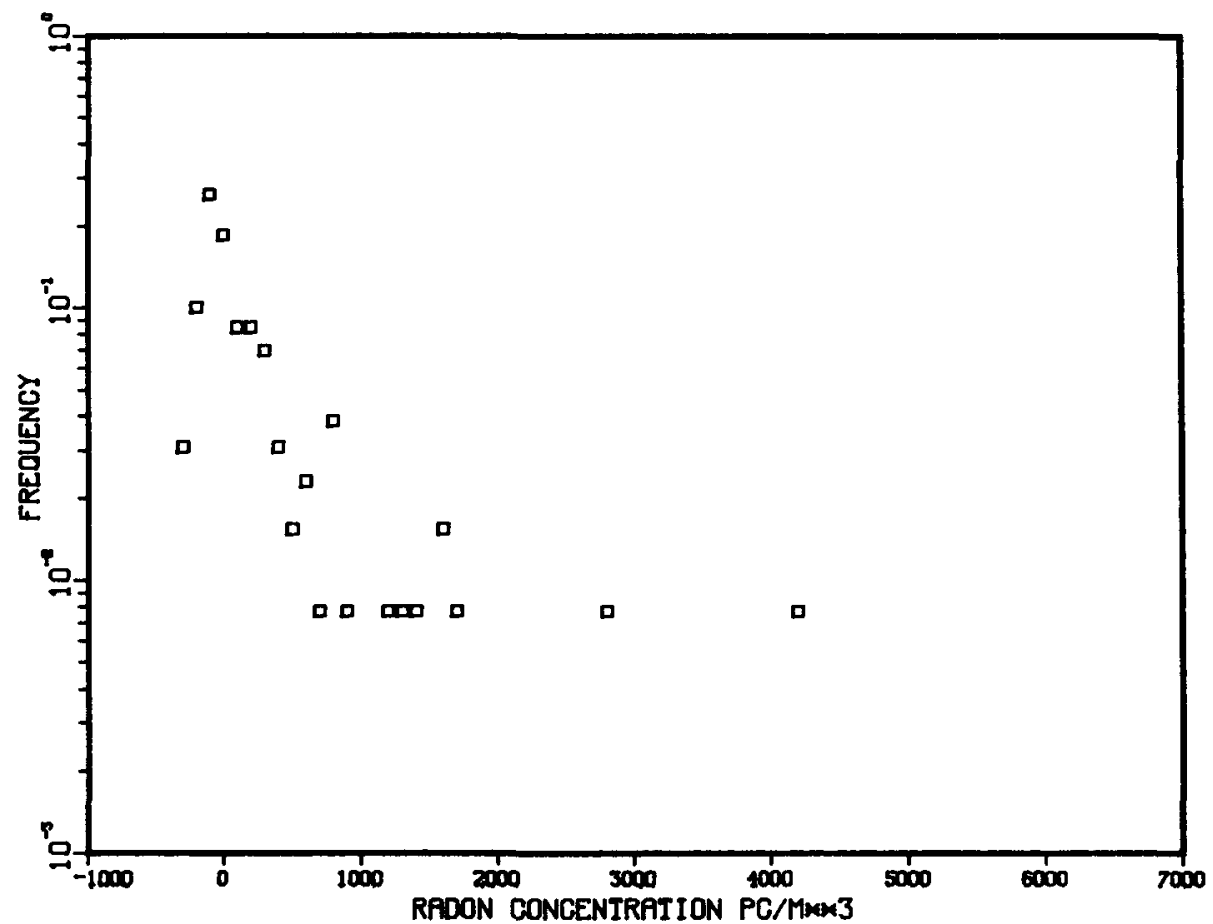

Figure A8. Continued. 
STATION 103, JULY 1977

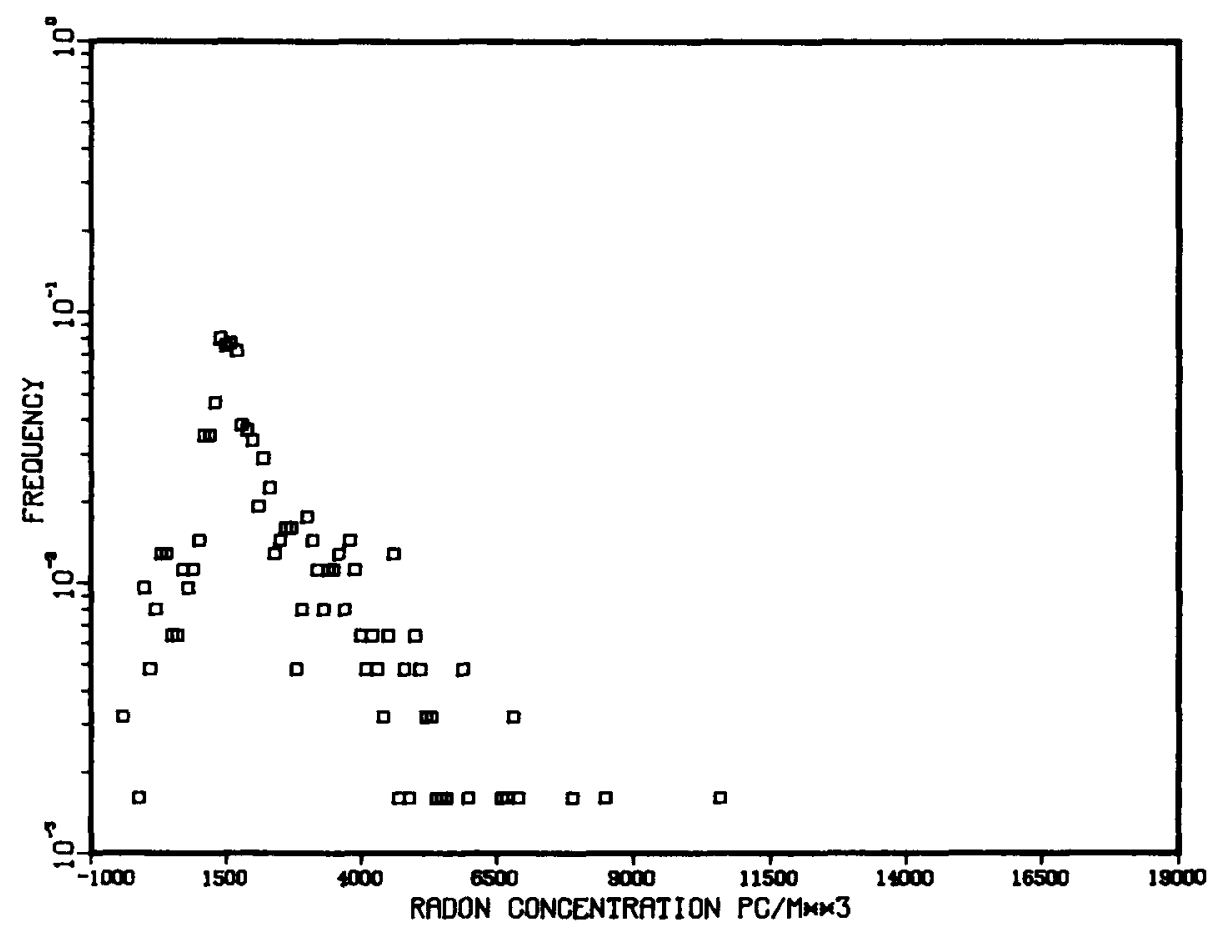

STATION 103, AUGUST 1977

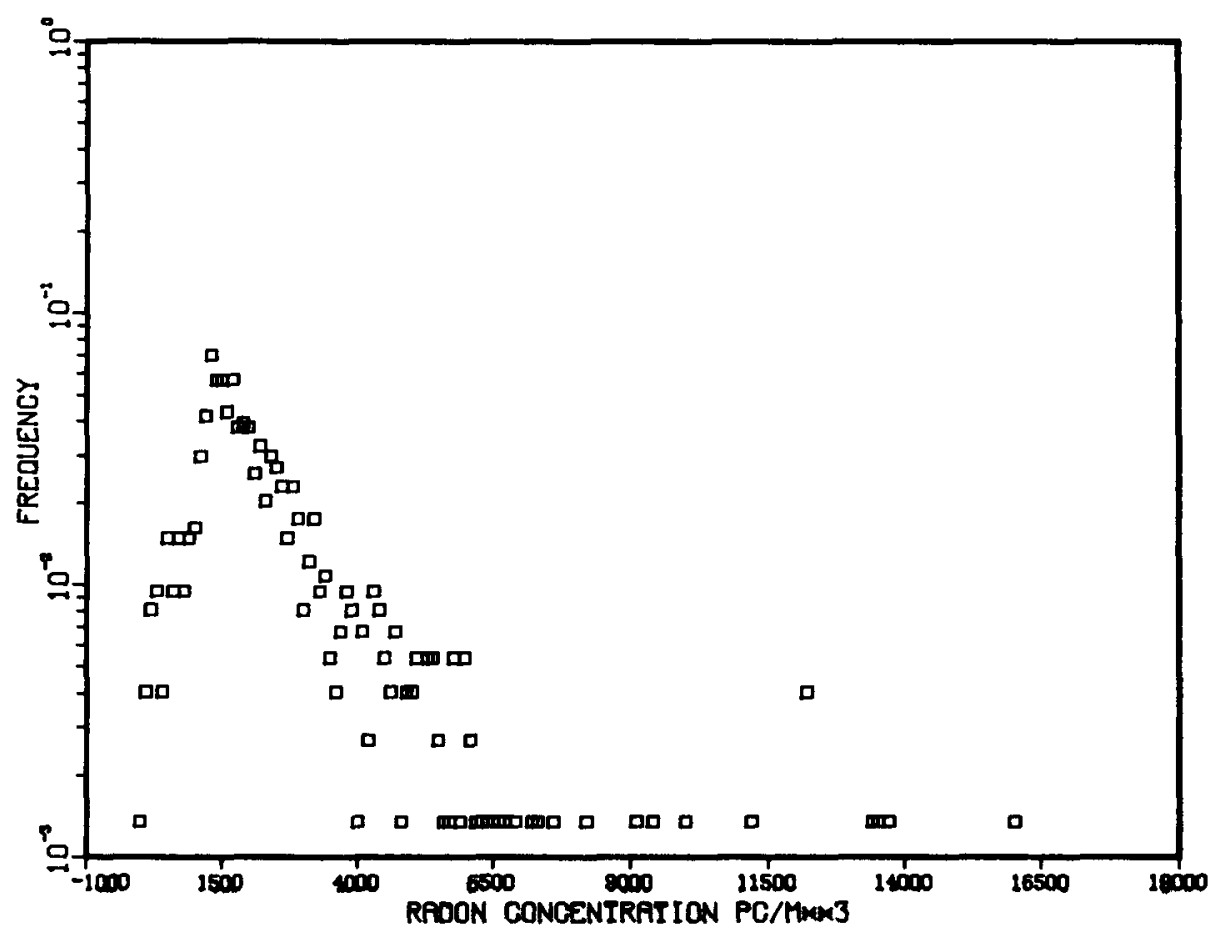

Figure A9. Radon-Concentration Frequency at Station 103. 
STATION 103, SEPTEMBER 1977

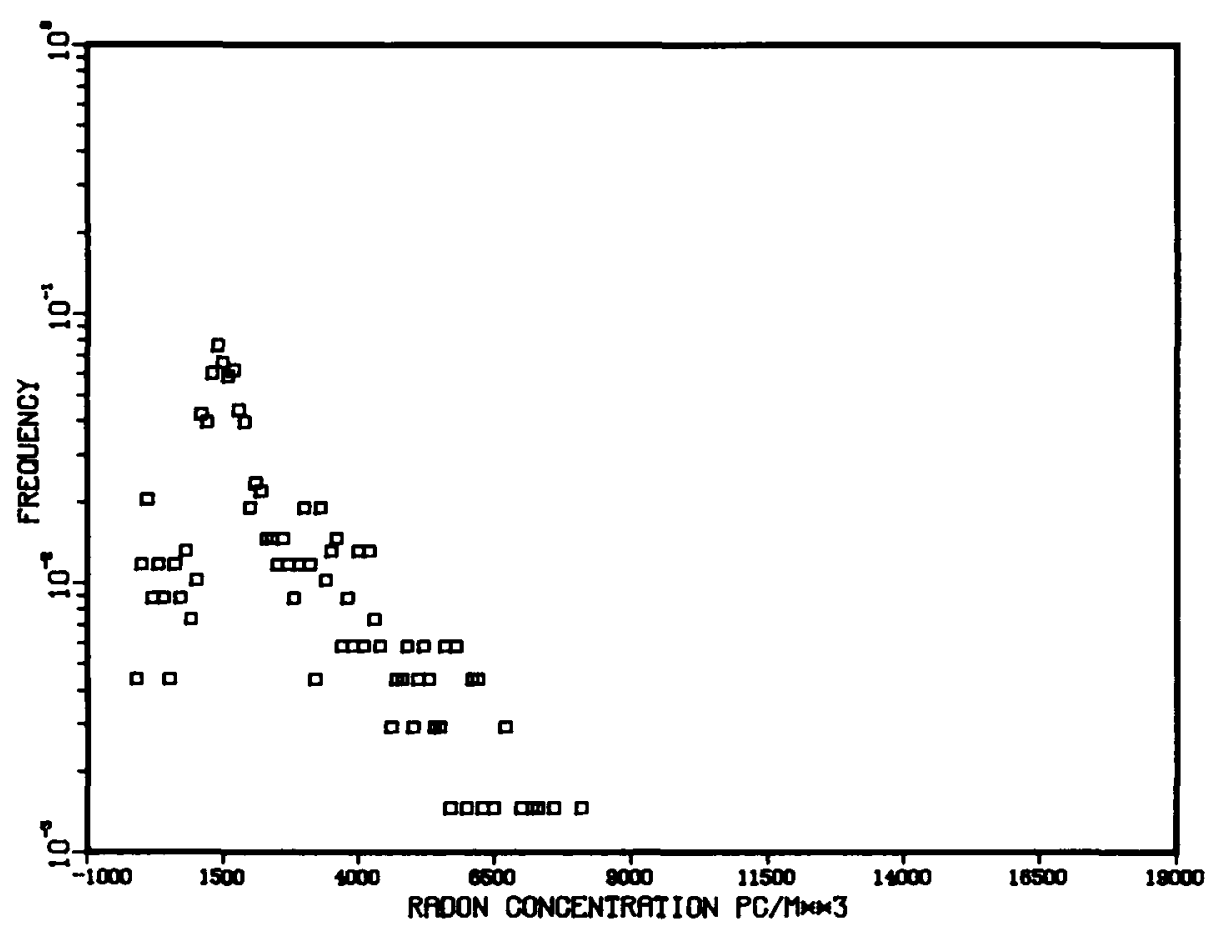

STATION 103, OCTOBER 1977

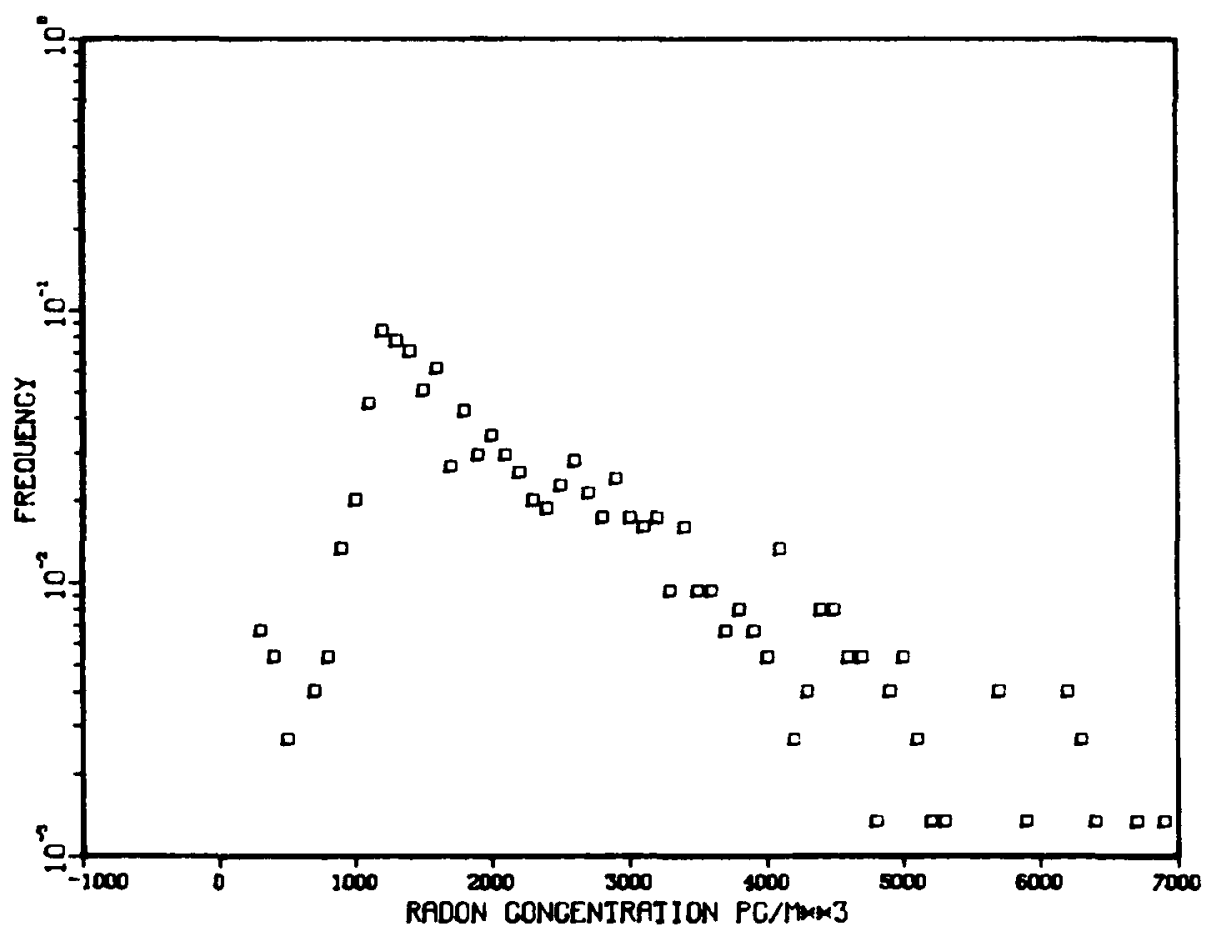

Figure A9. Continued. 
STATION 103, NOVEMBER 1977

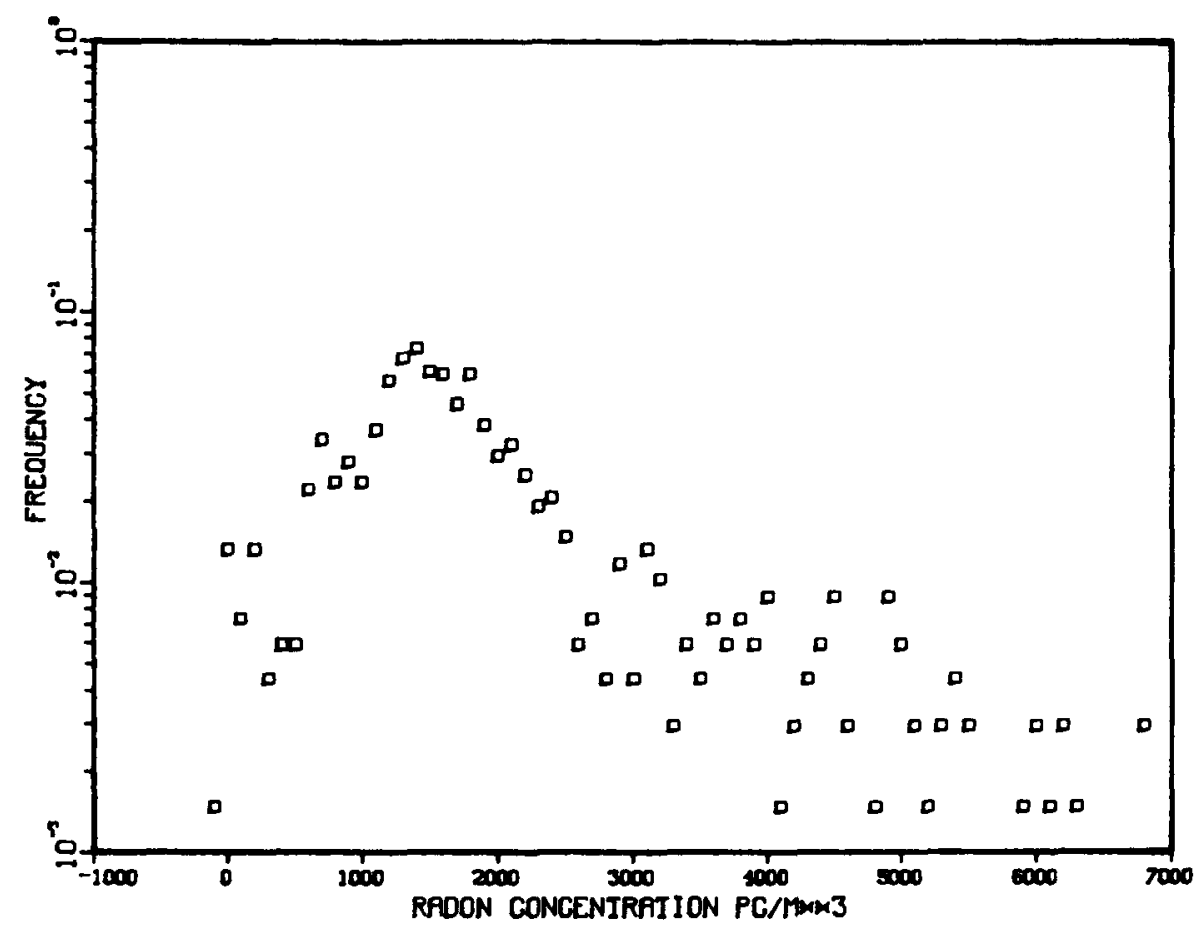

STATION 103, DECEMBER 1977

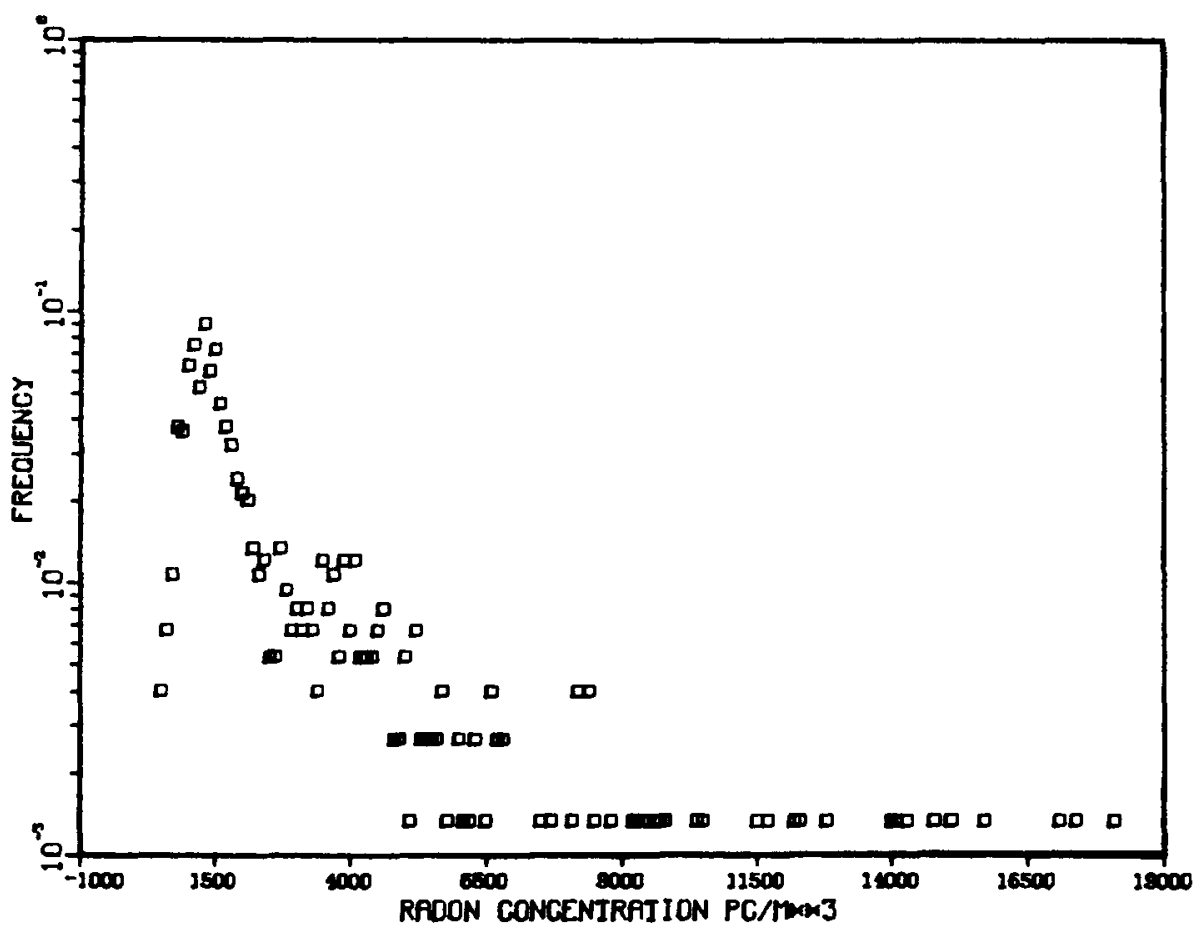

Figure A9. Continued. 


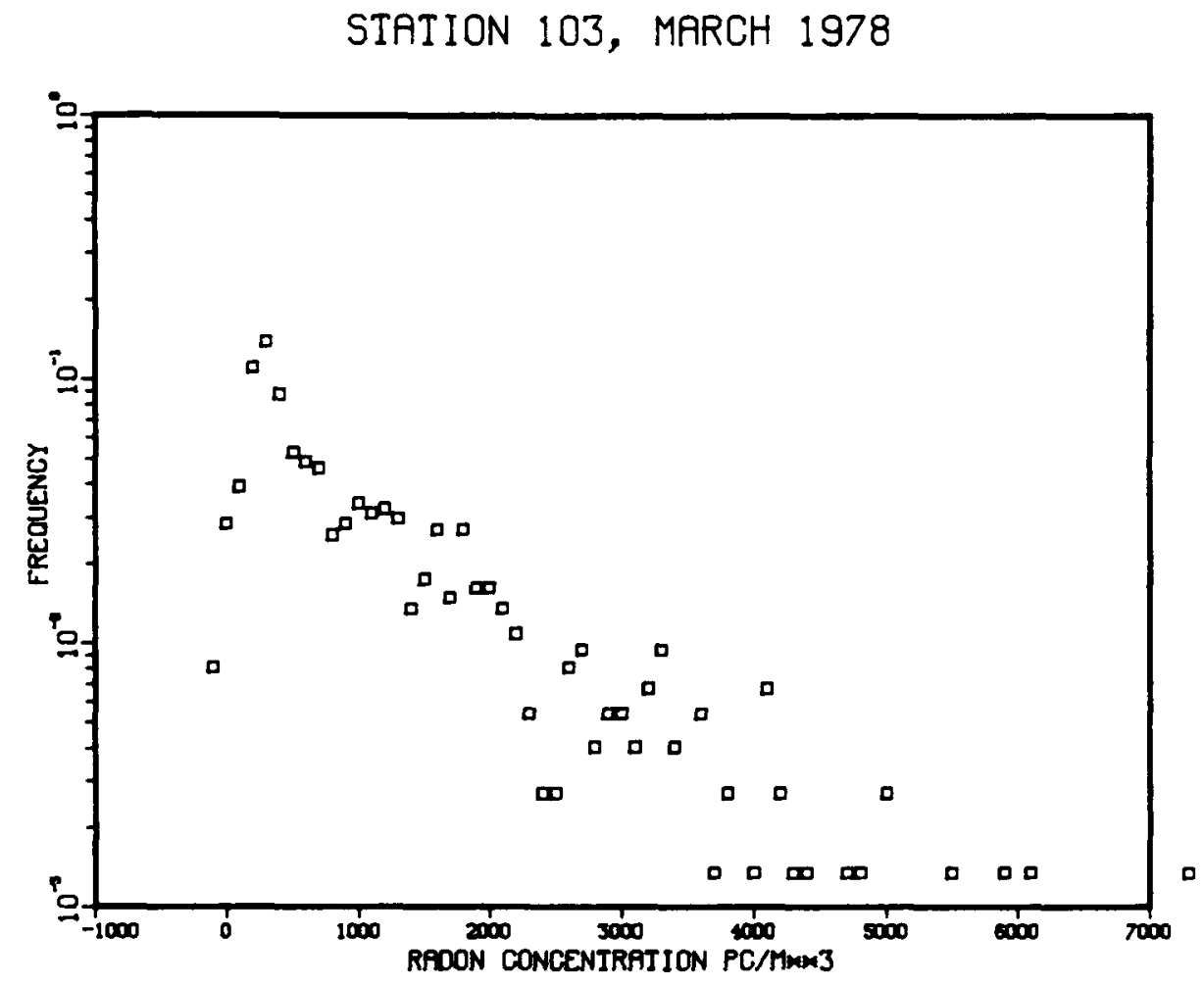

STATION 1C3, APRIL 1978

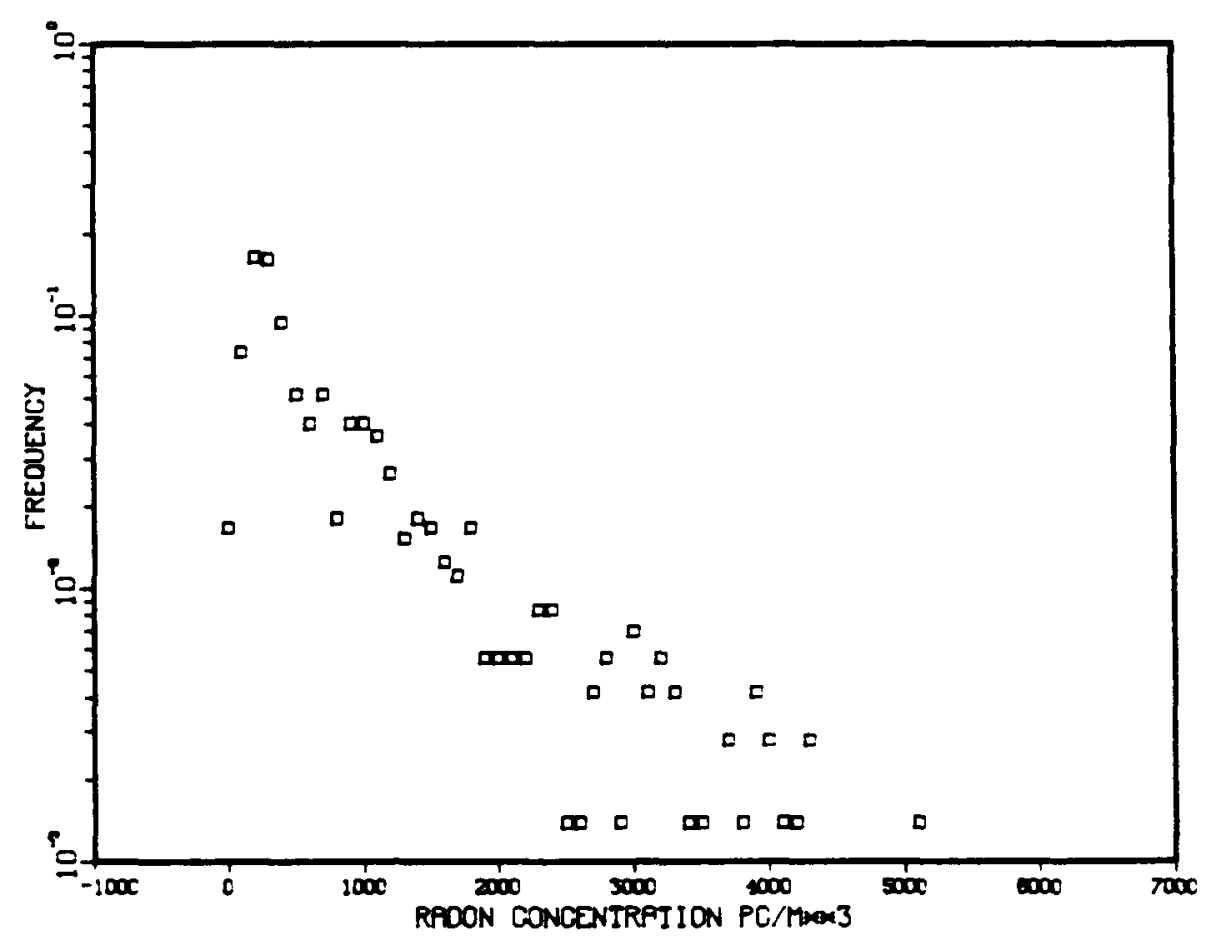

Figure A9. Continued. 
STATION 103, MAY 1978

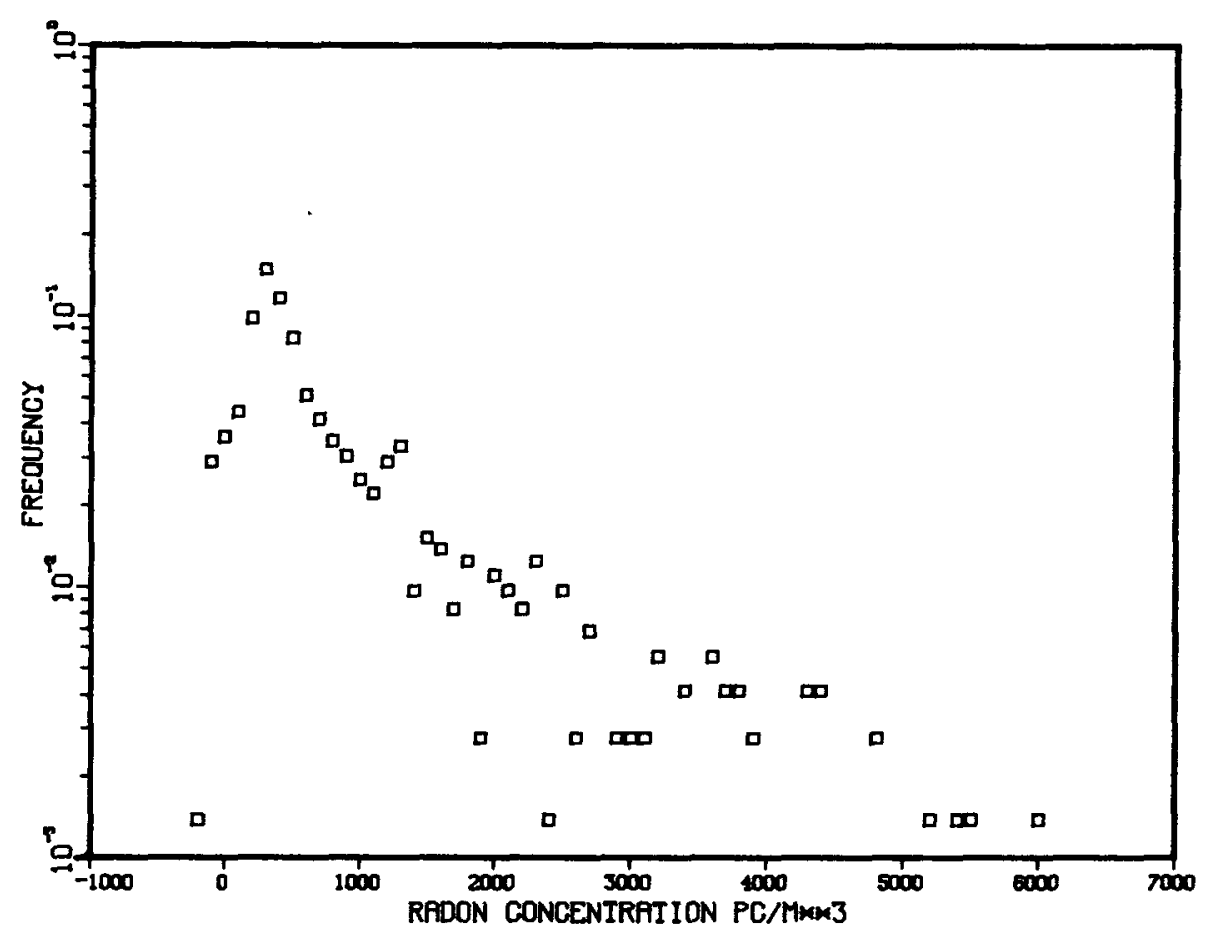

STATION 103, JUNE 1978

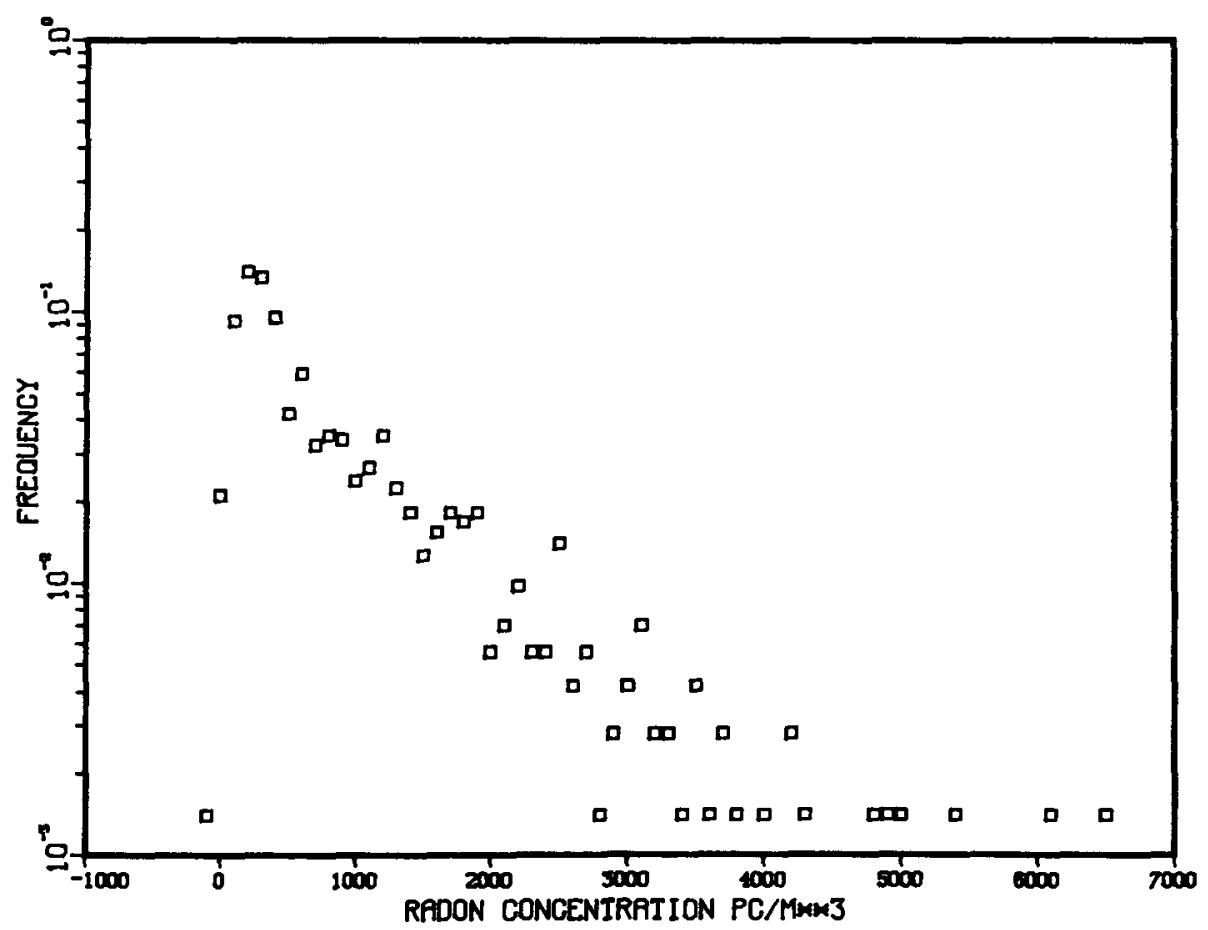

Figure A9. Continued. 
STATION 103, JULY 1978

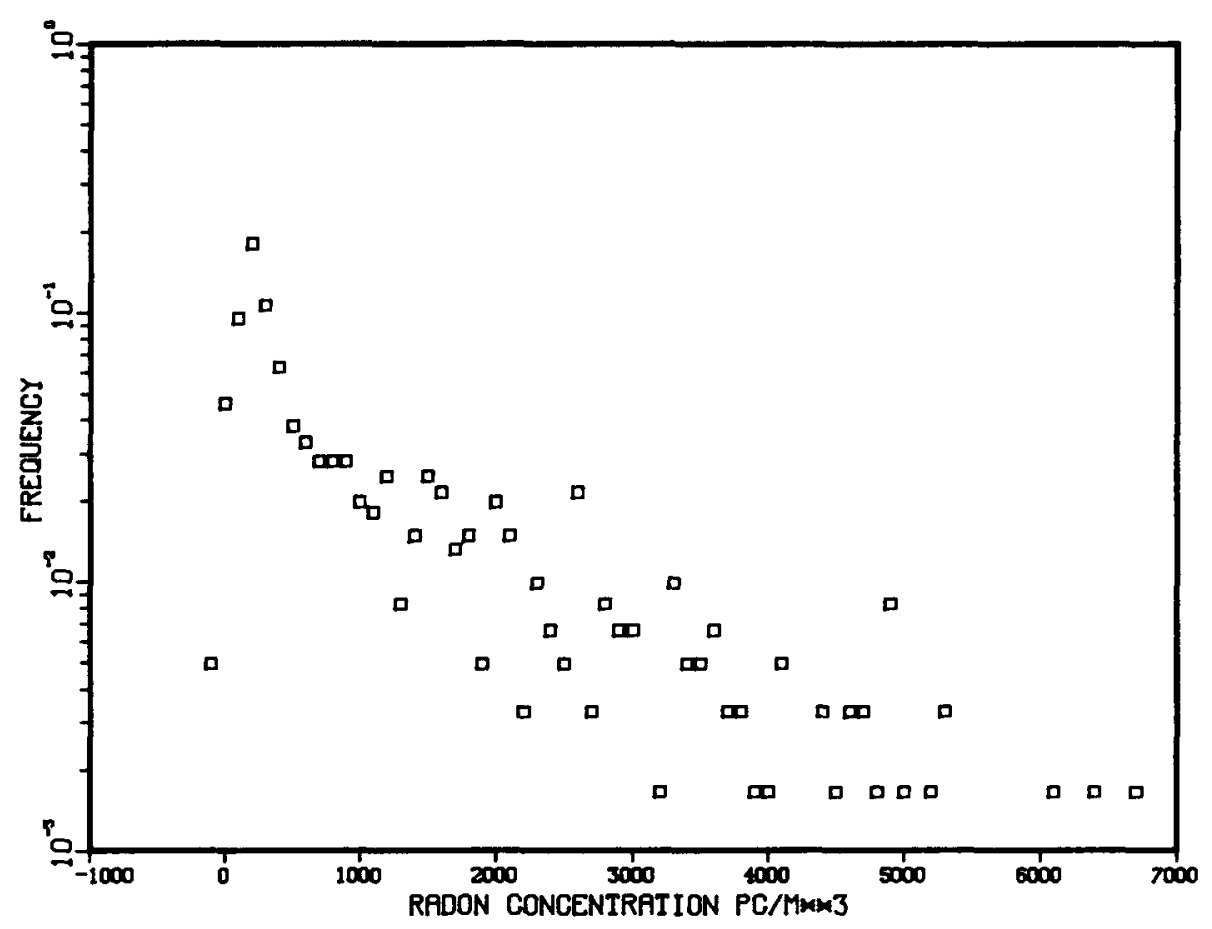

Figure A9. Continued. 

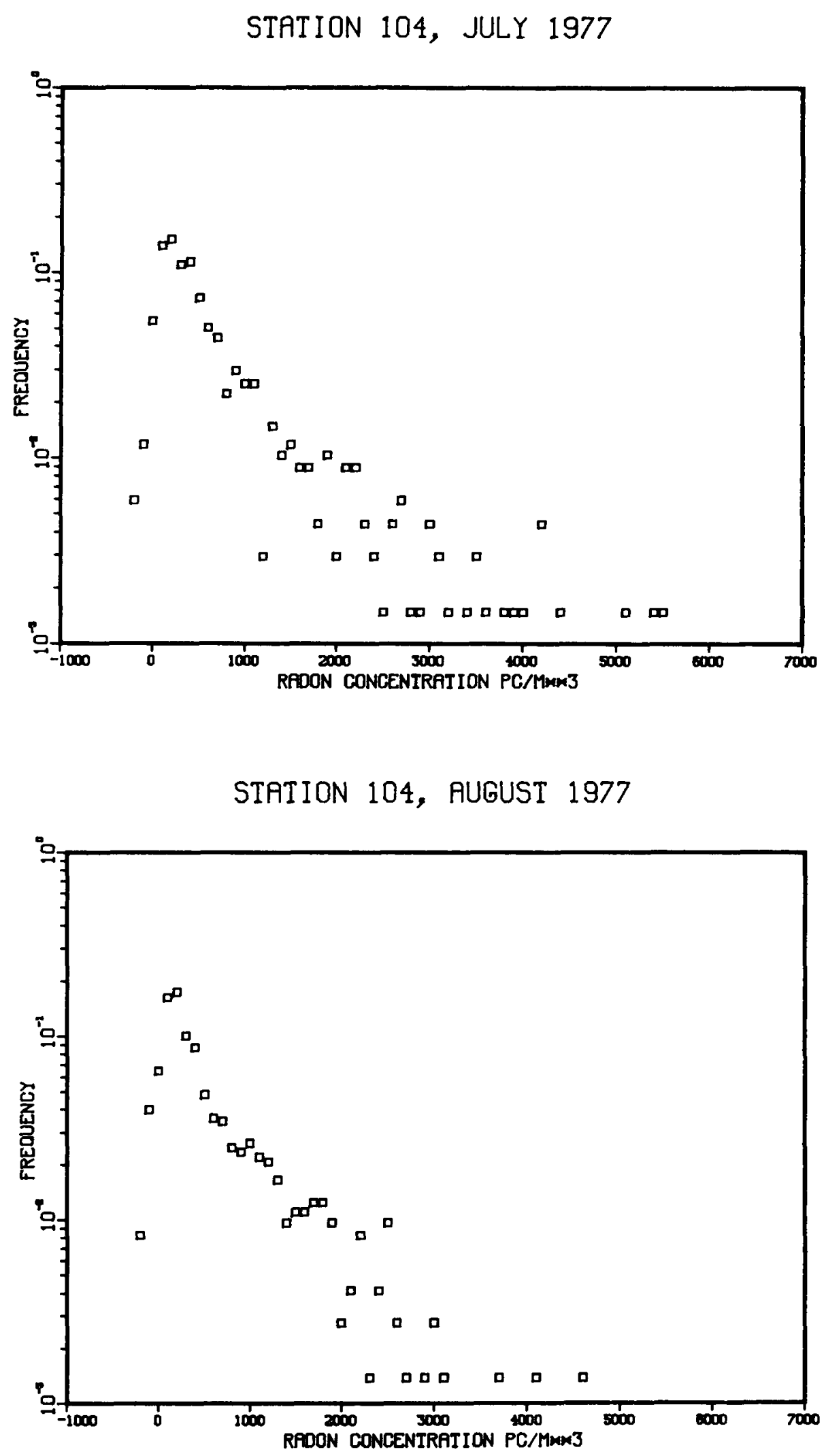

Figure A10. Radon-Concentration Frequency at Station 104. 
STATION 104, SEPTEMBER 1977

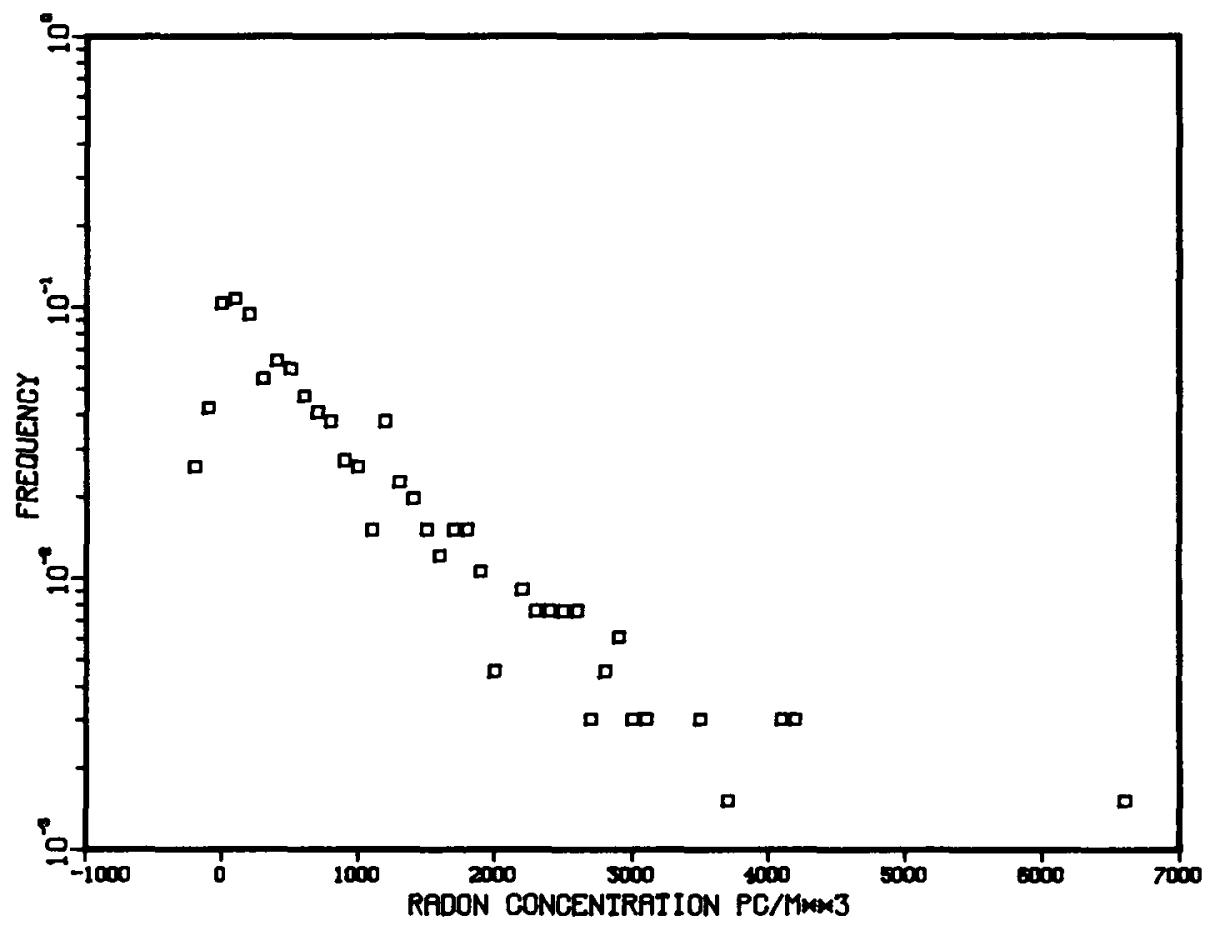

STATION 104, OCTOBER 1977

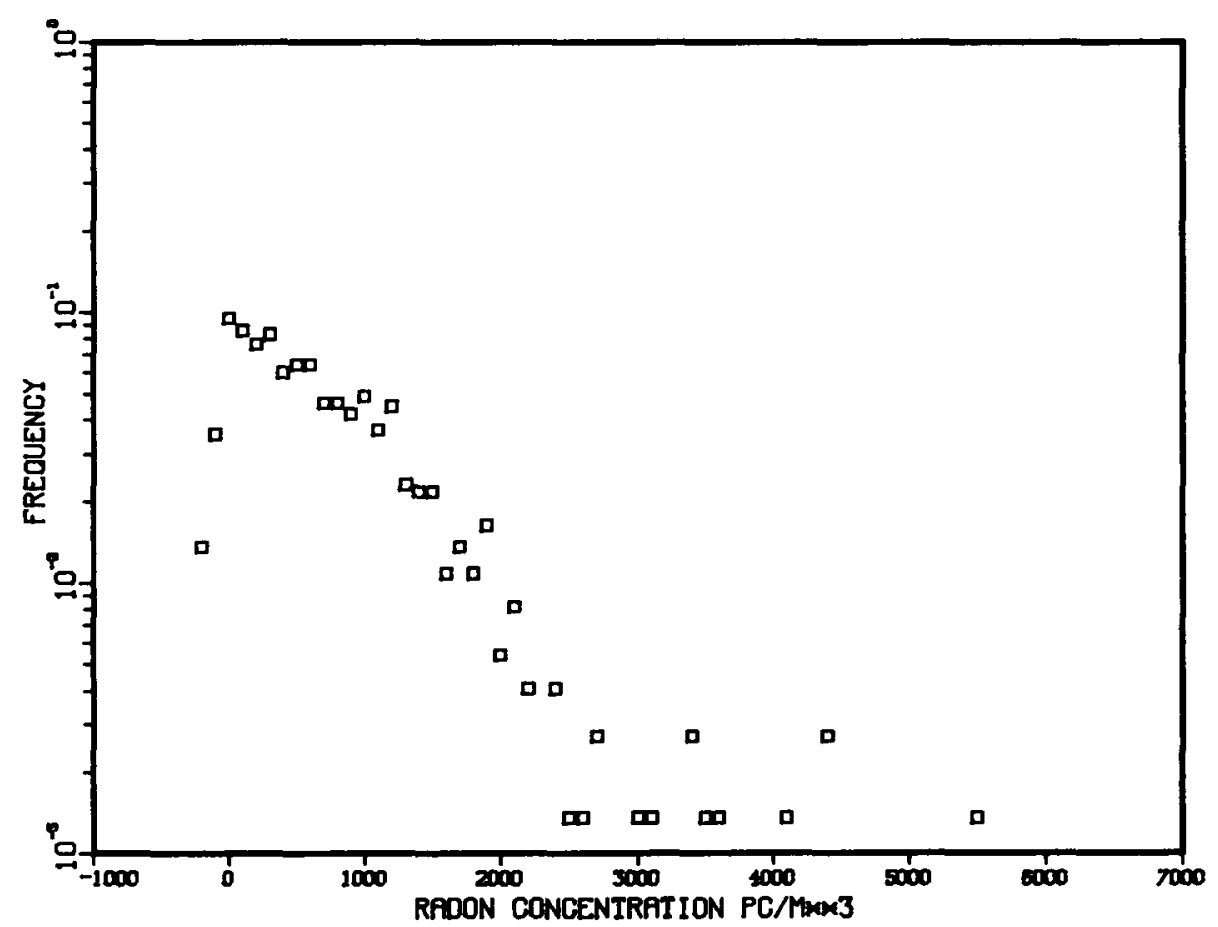

Figure Alo. Continued. 
STATION 104, NOVEMBER 1977

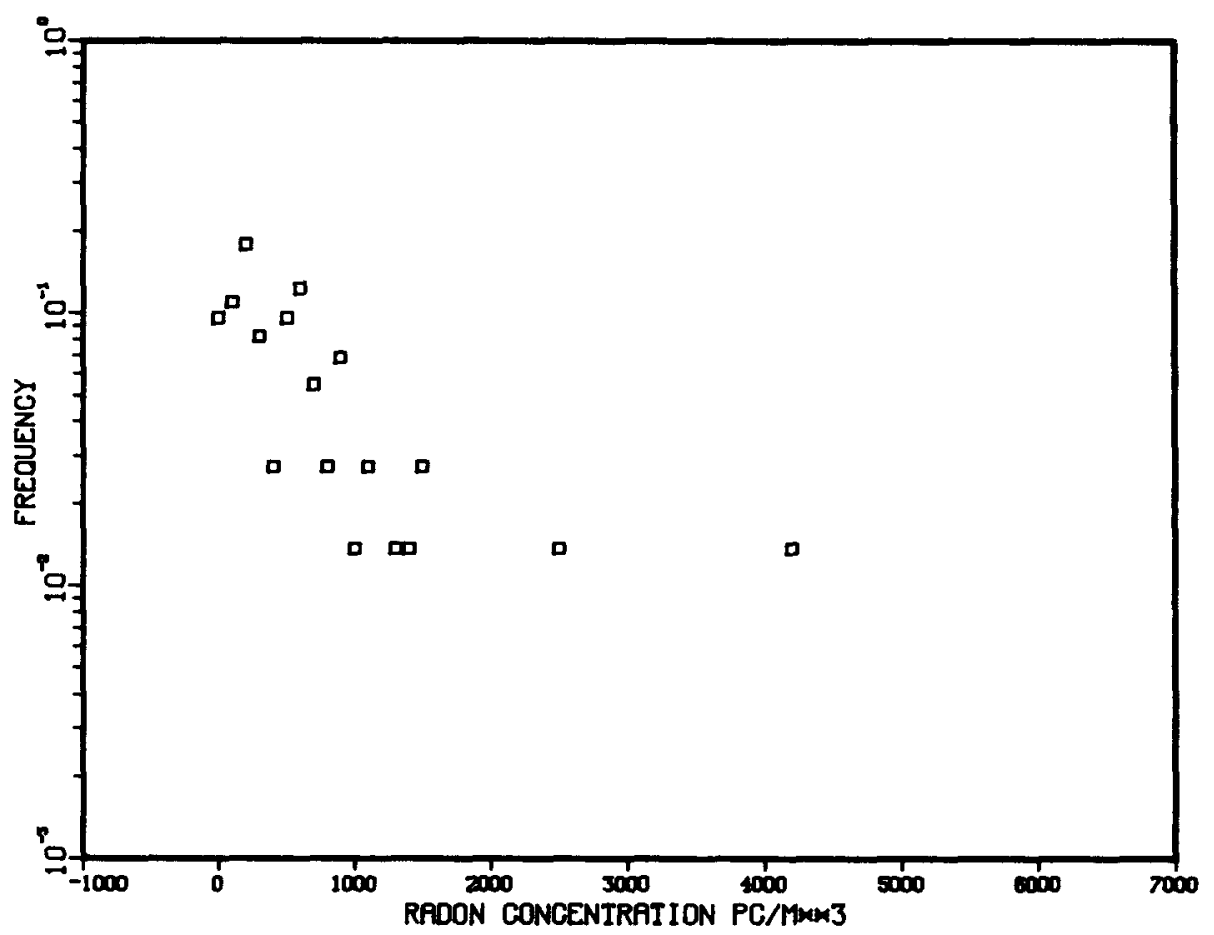

STATION 104, DECEMBER 1977

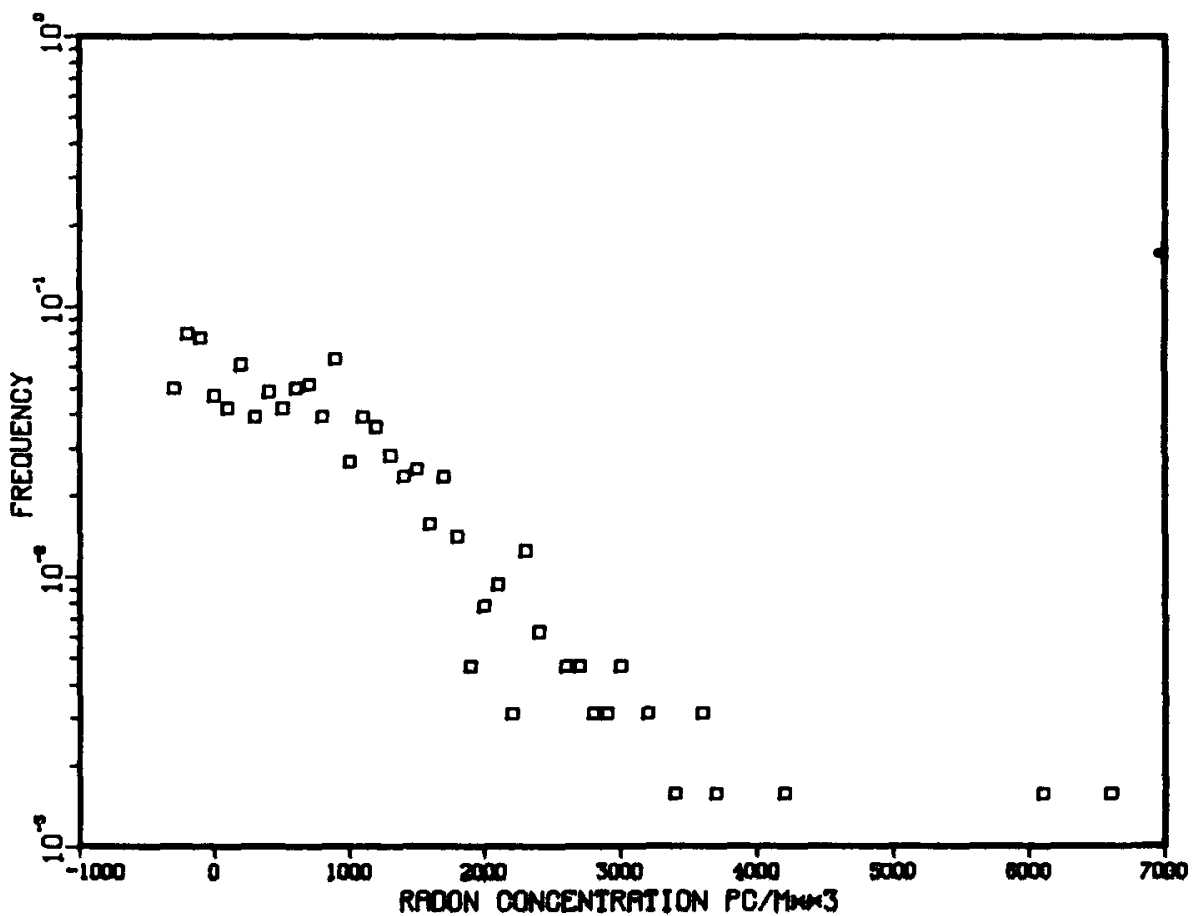

Figure Alo. Continued. 
STATION 104, MARCH 1978

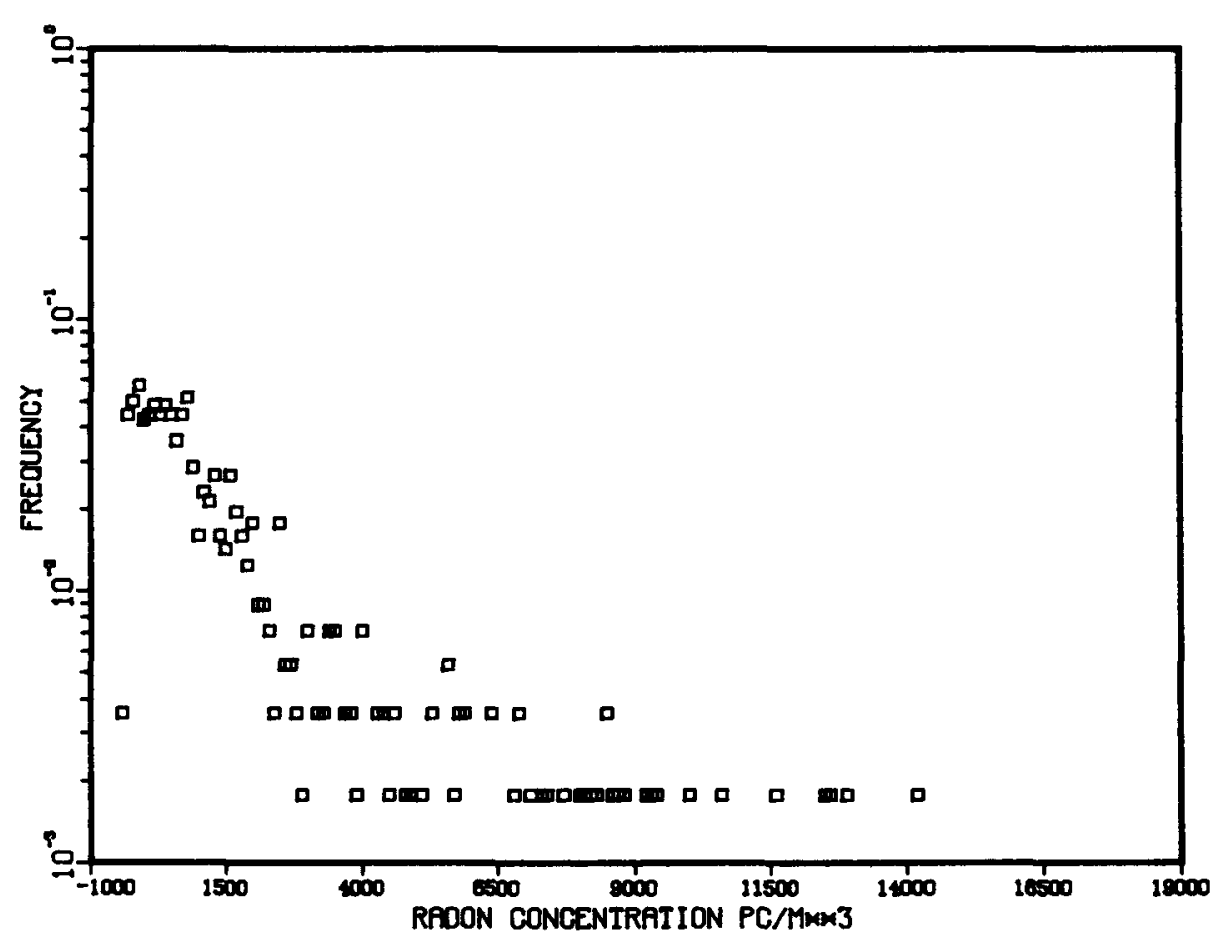

Figure Alo. Continued. 
STATION 102, JULY 1977

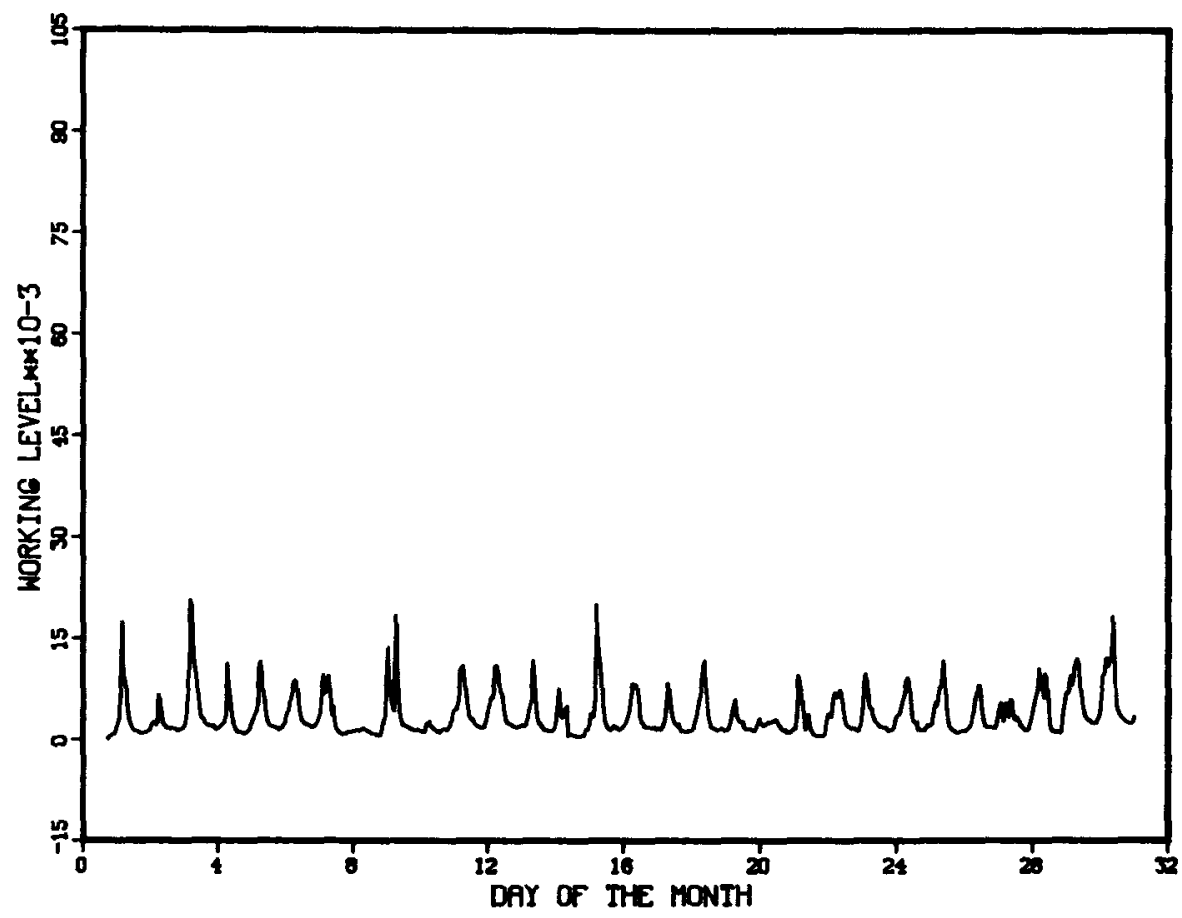

STATION 102, AUGUST 1977

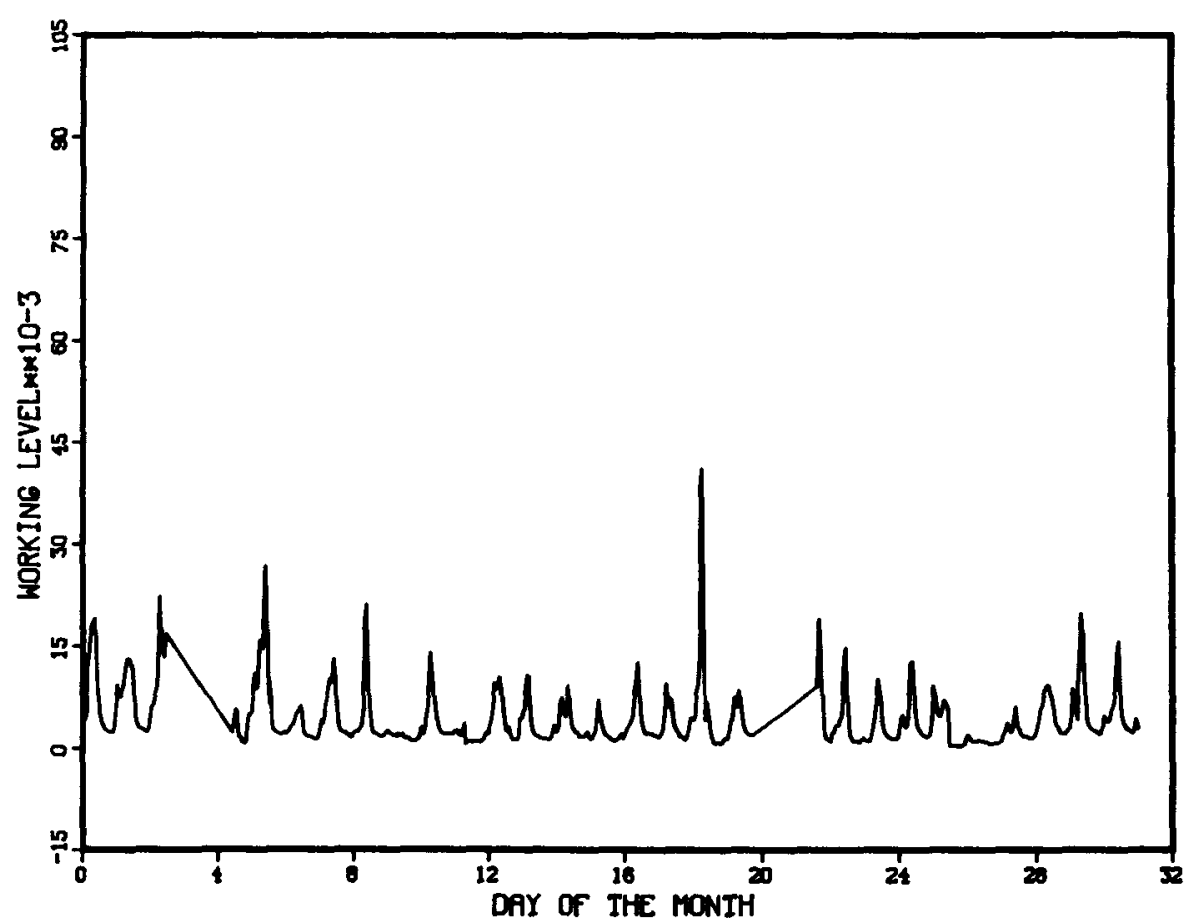

Figure All. Working Level by Hour at Station 102 . 
STATION 102, SEPTEMBER 1977

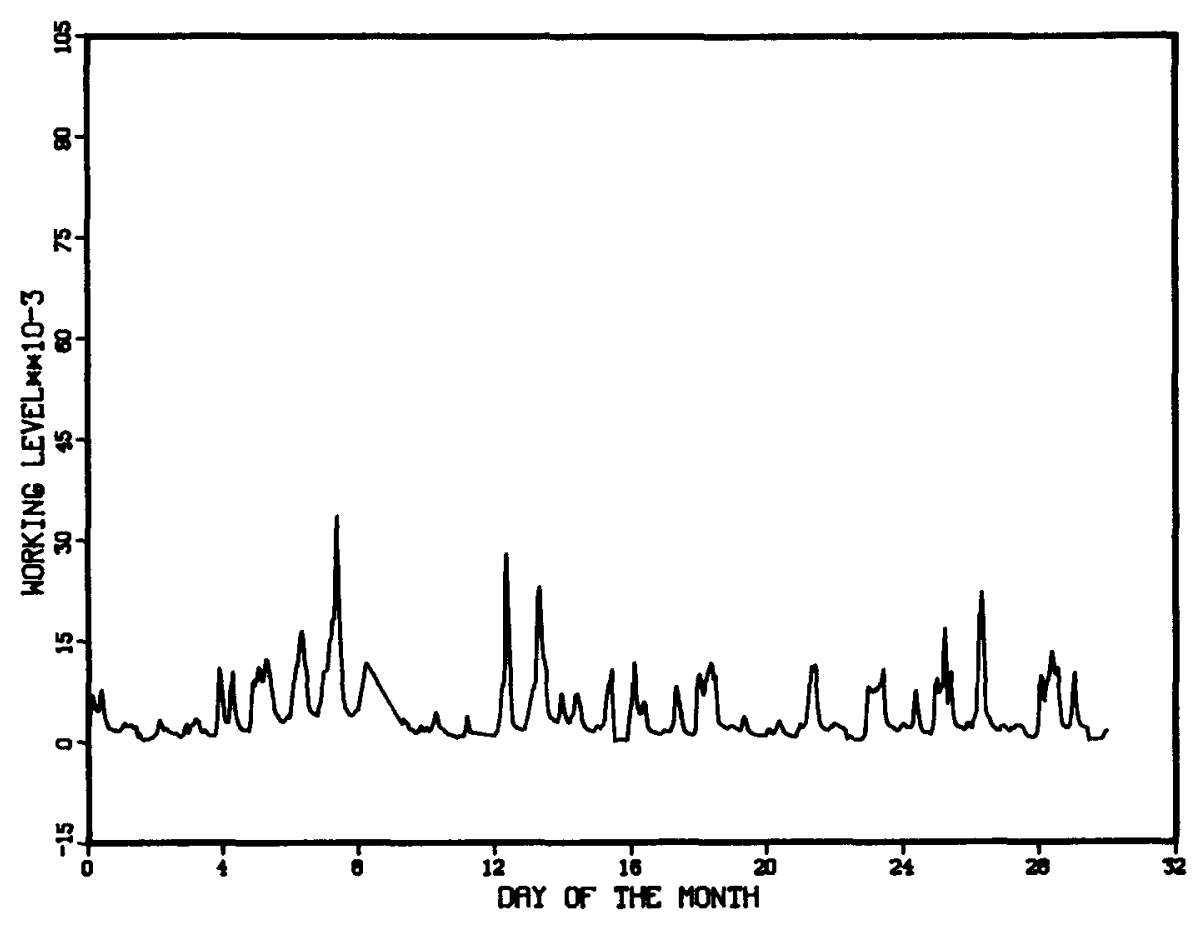

STATION 102, OCTOBER 1977

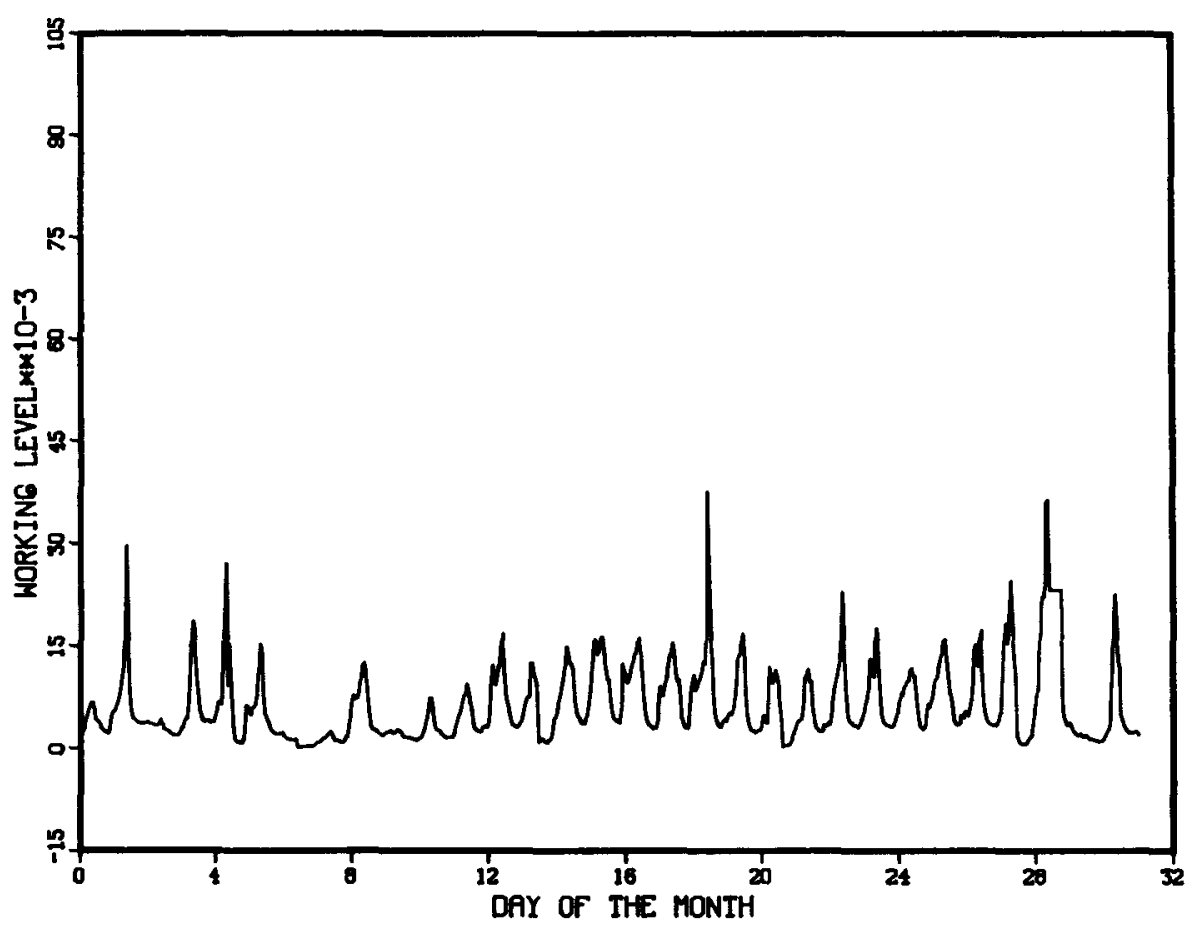

Figure All. Continued. 
STATION 102, NOVEMBER 1977

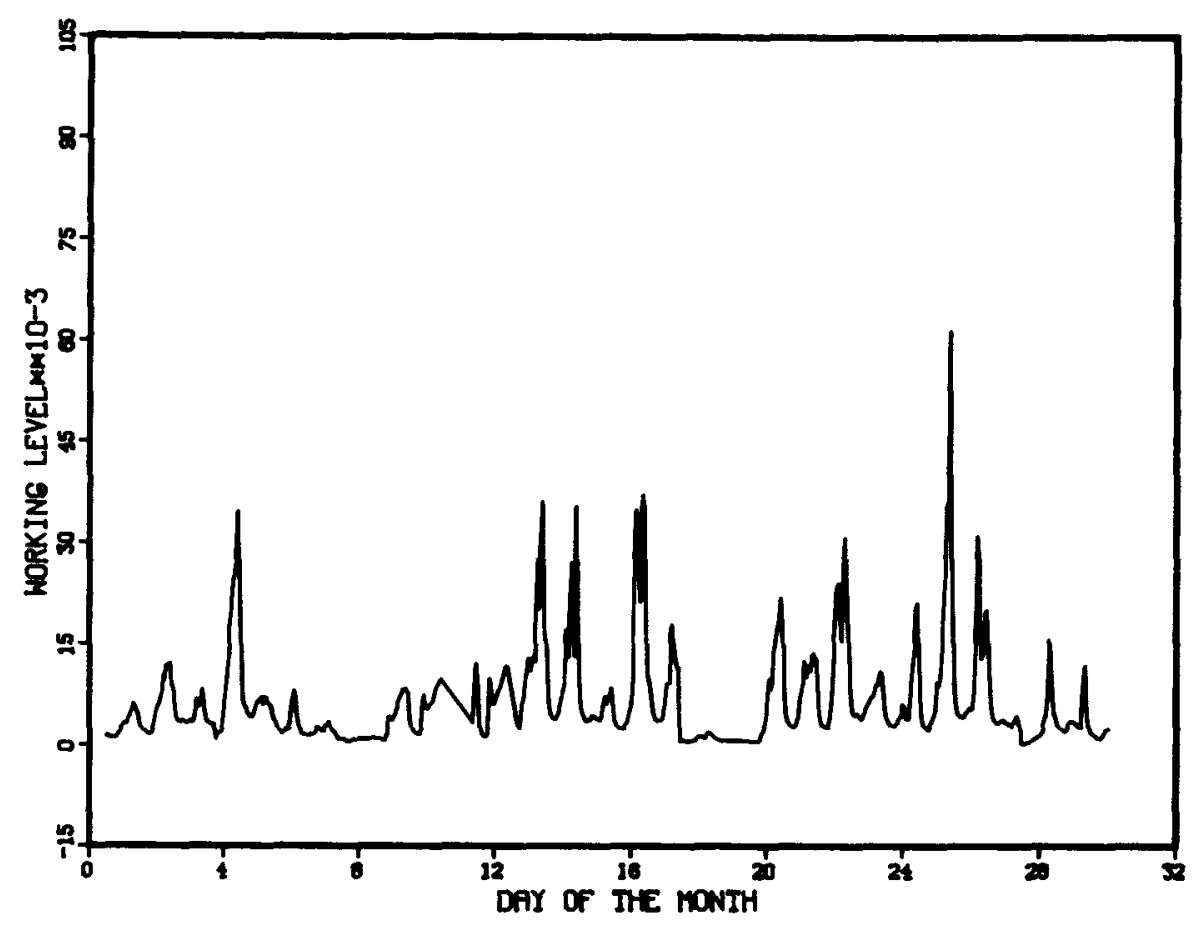

STATION 102, DECEMBER 1977

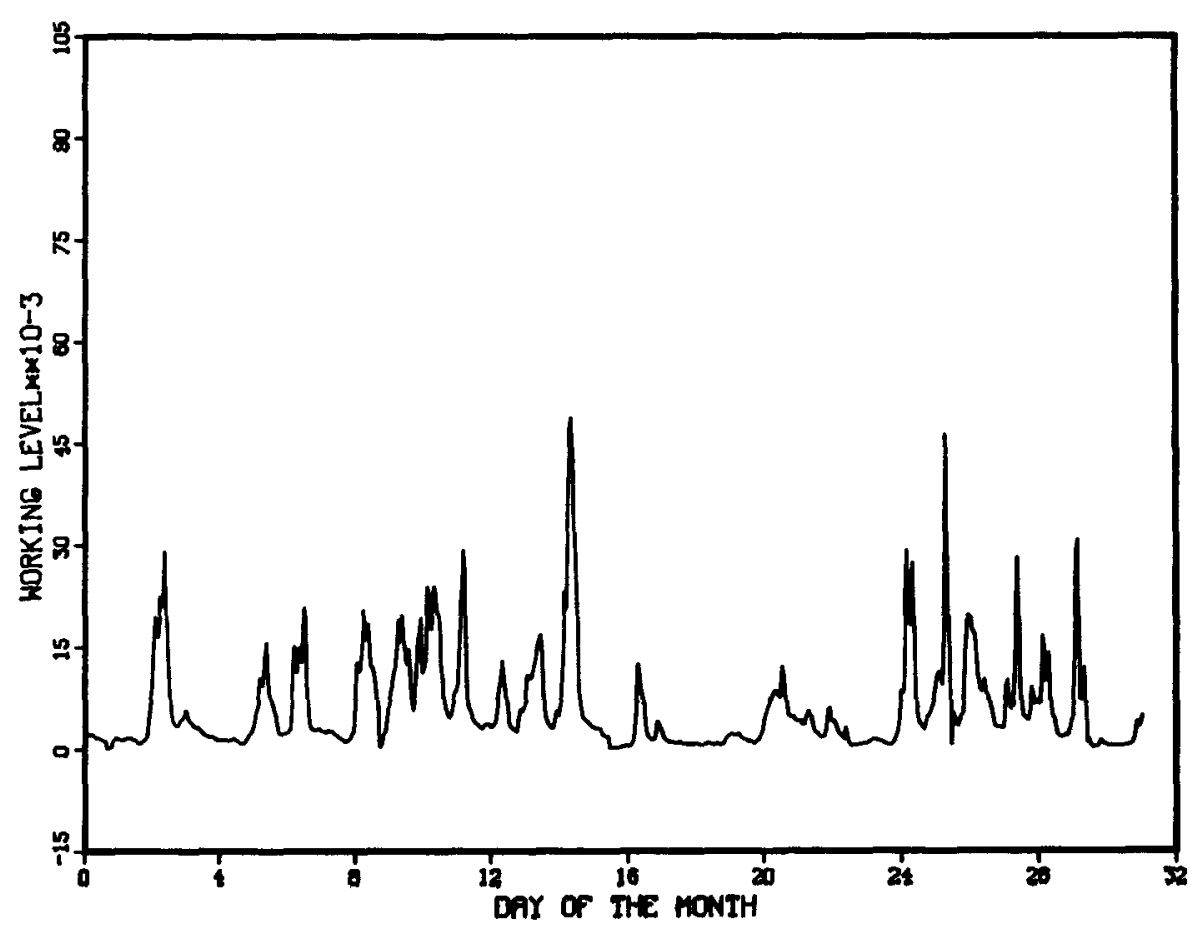

Figure All. Continued. 
STATION 102, MARCH 1978

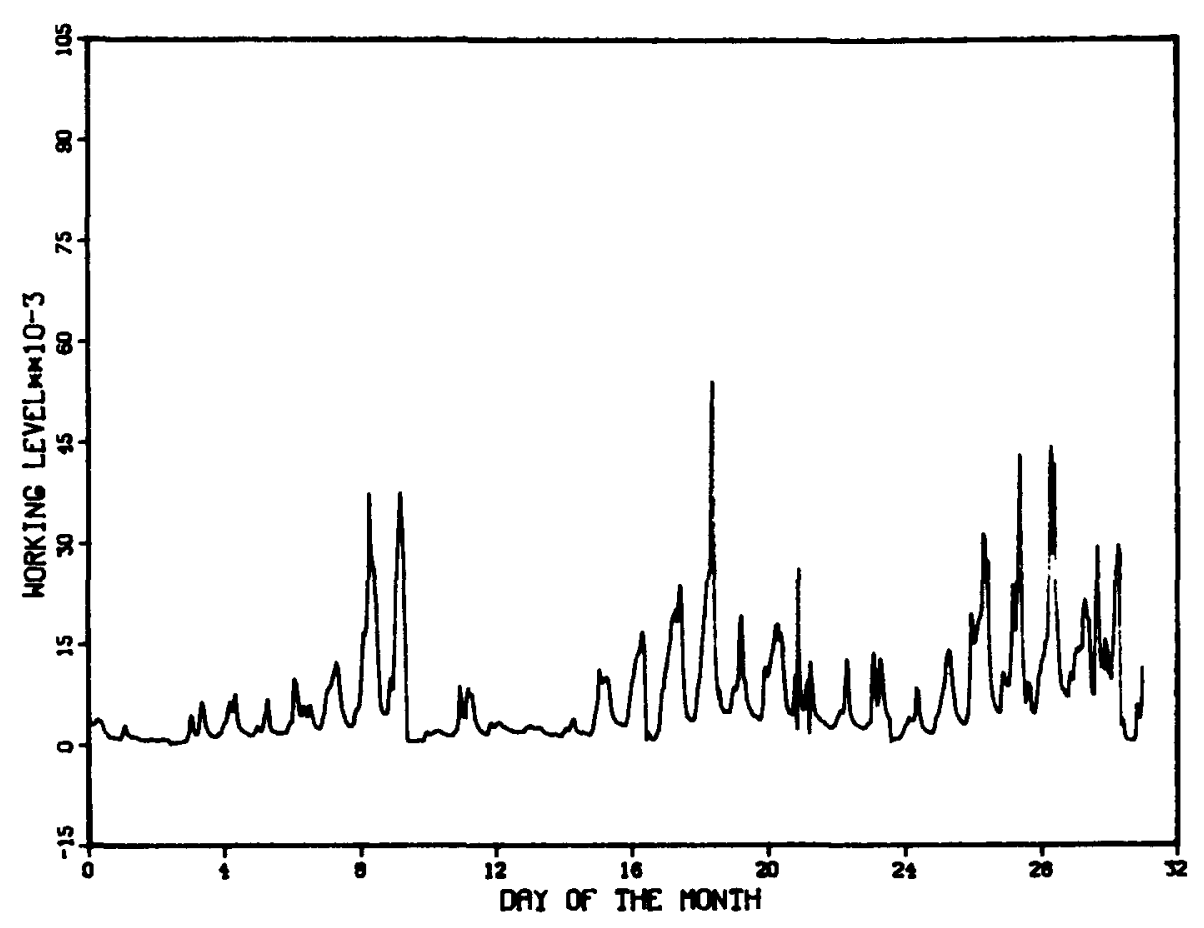

STATION 102, APRIL 1978

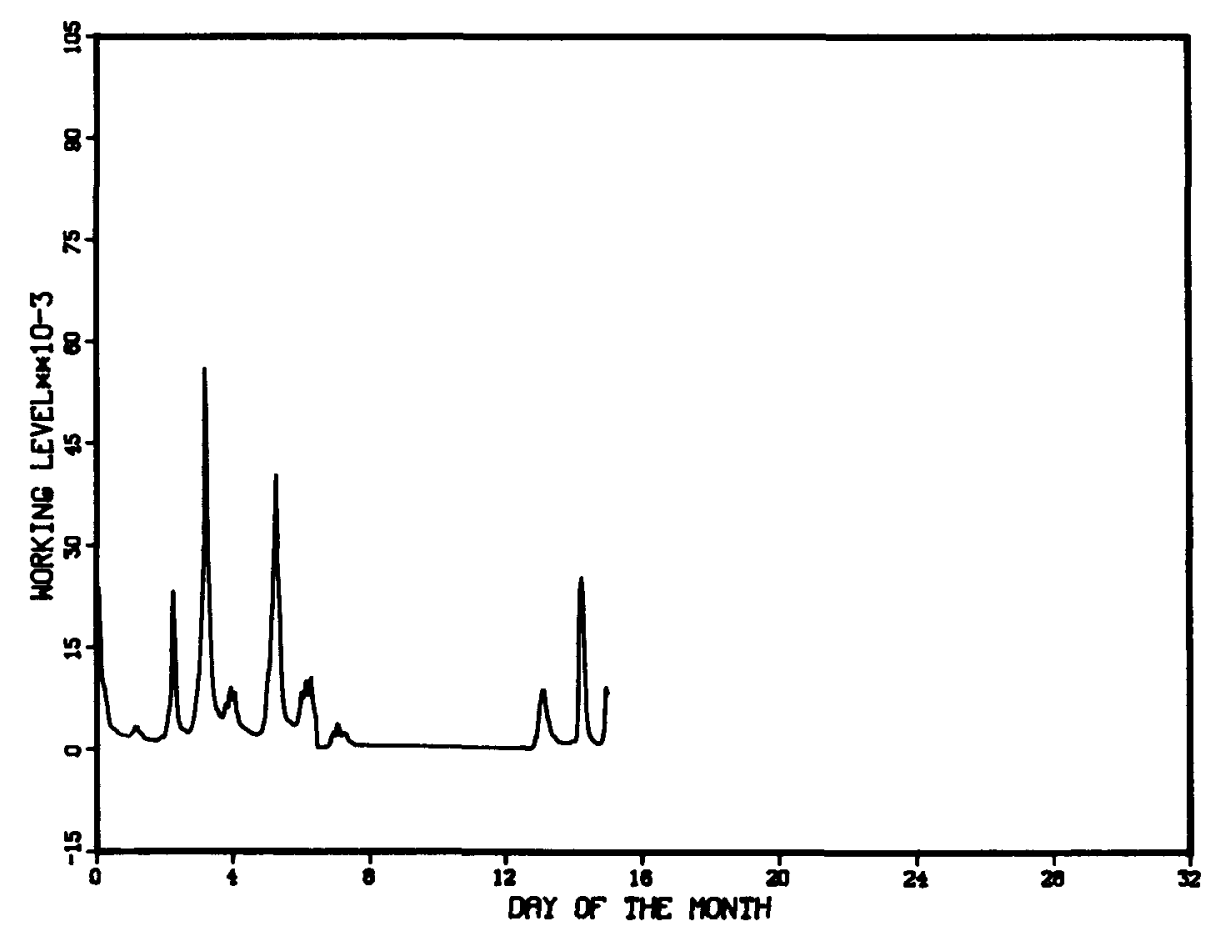

Figure All. Continued. 
STATION 102, JUNE 1978

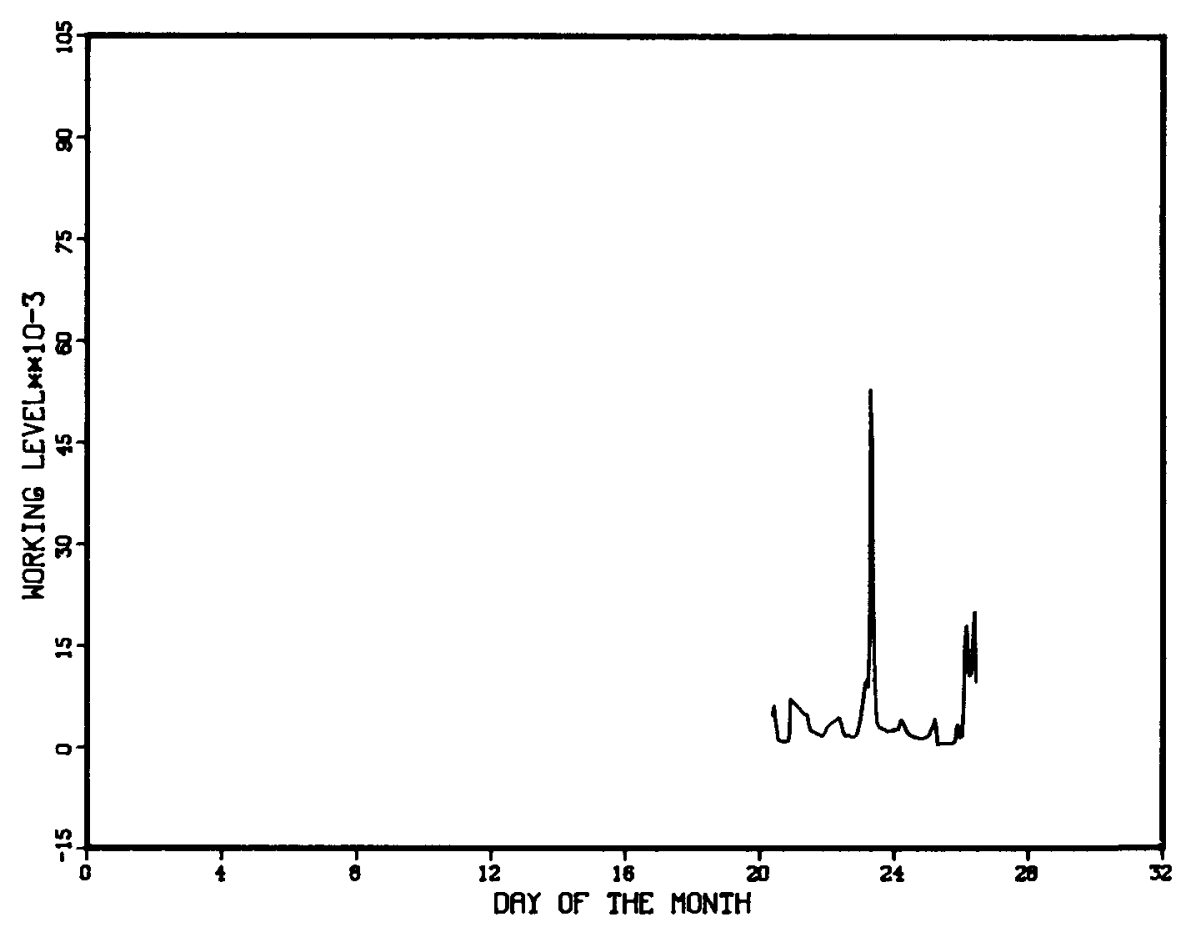

Figure All. Continued. 
STATION 103, JULY 1977

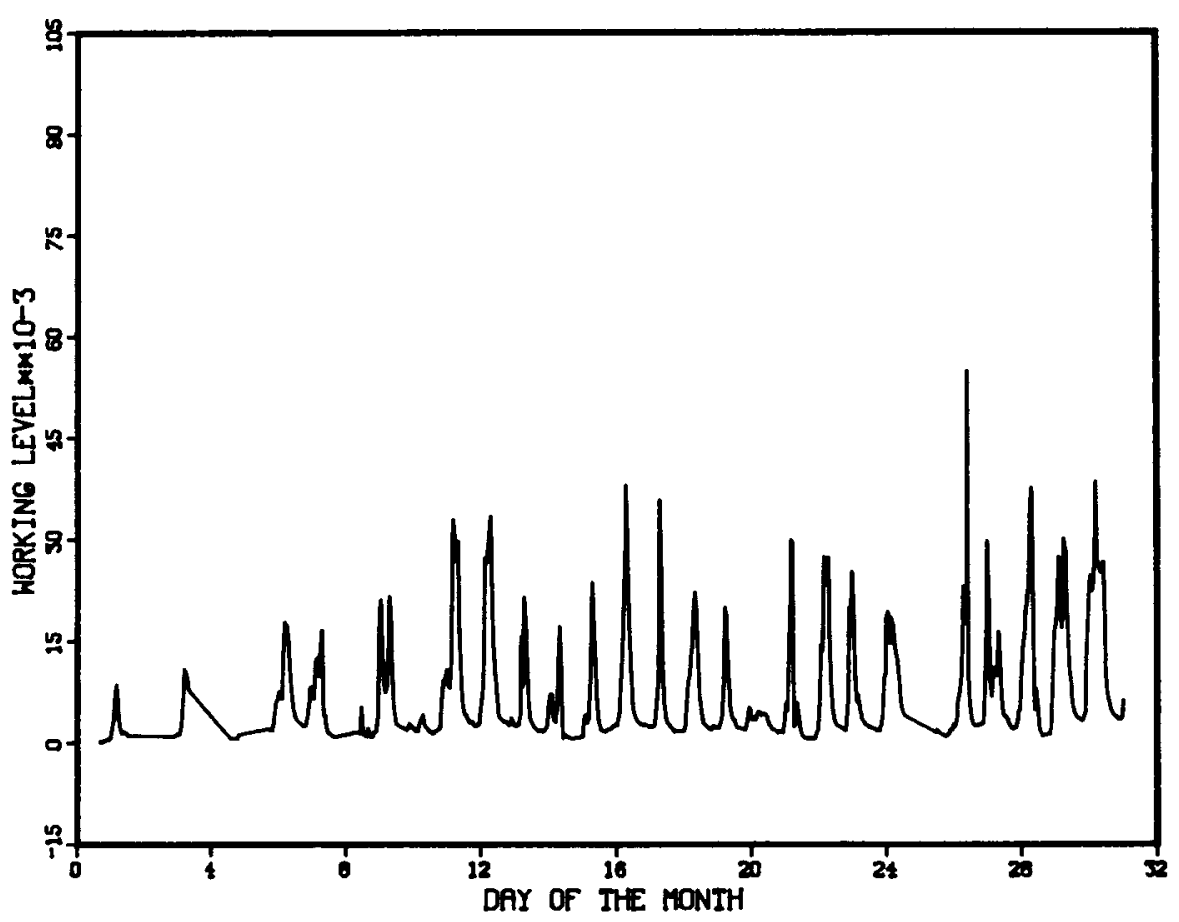

STATION 103, AUGUST 1977

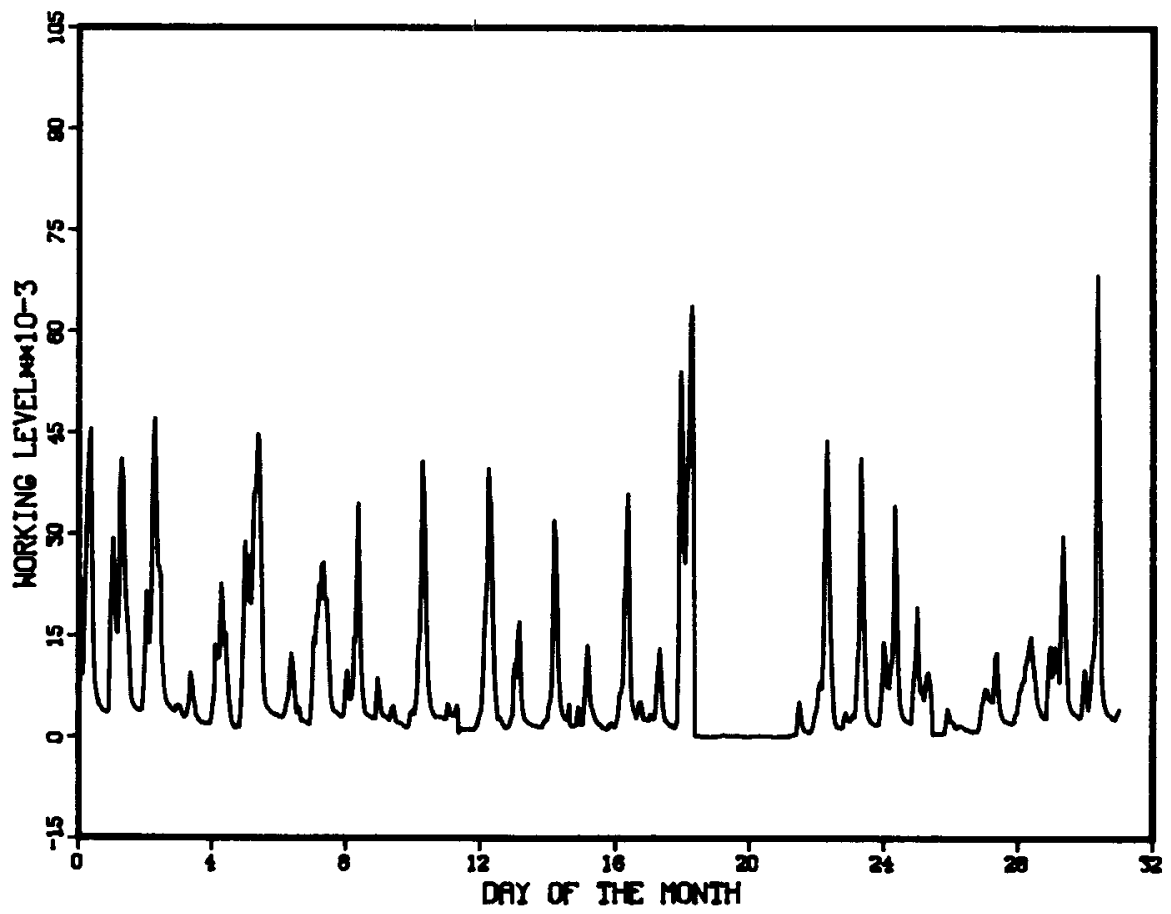

Figure A12. Working Level by Hour at Station 103. 
STATION 103, SEPTEMBER 1977

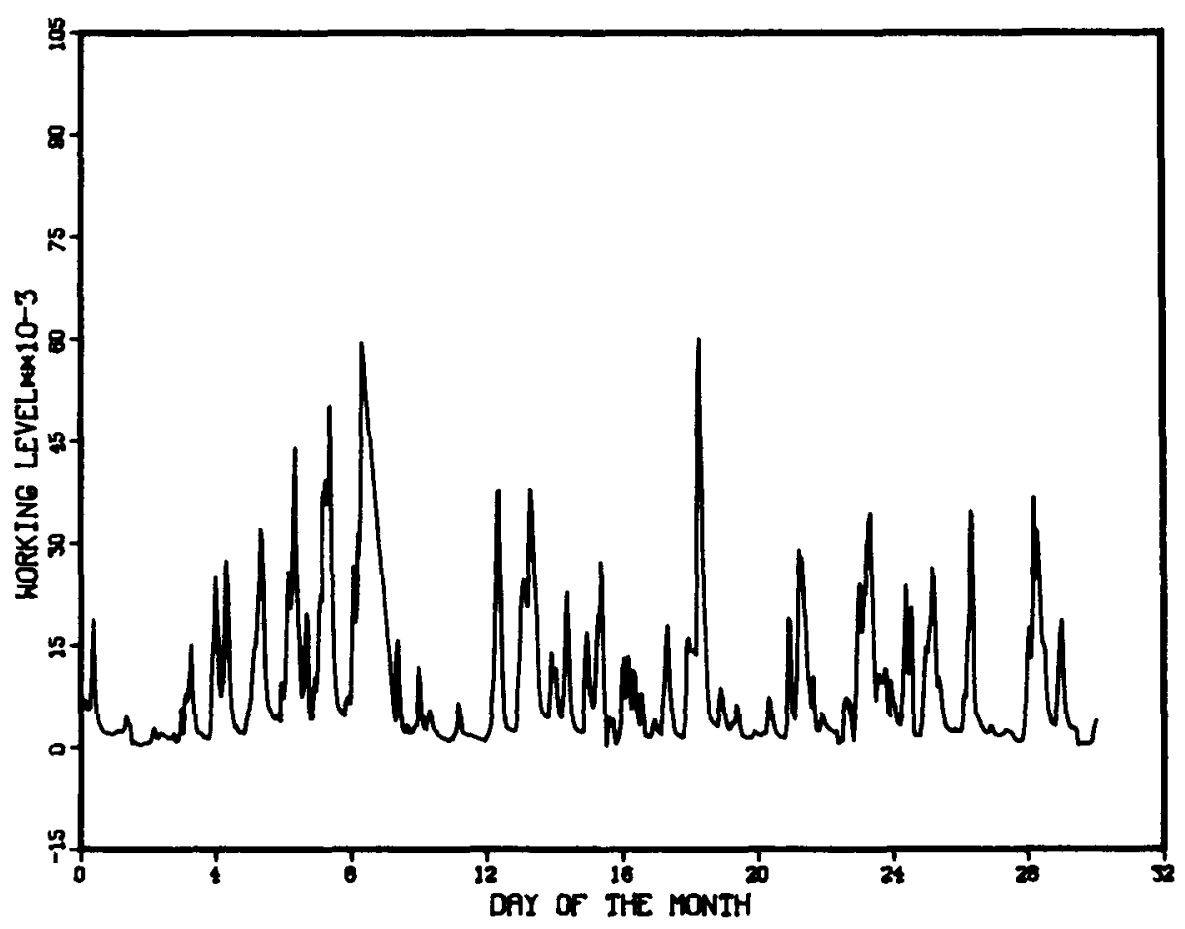

STATION 103, OCTOBER 1977

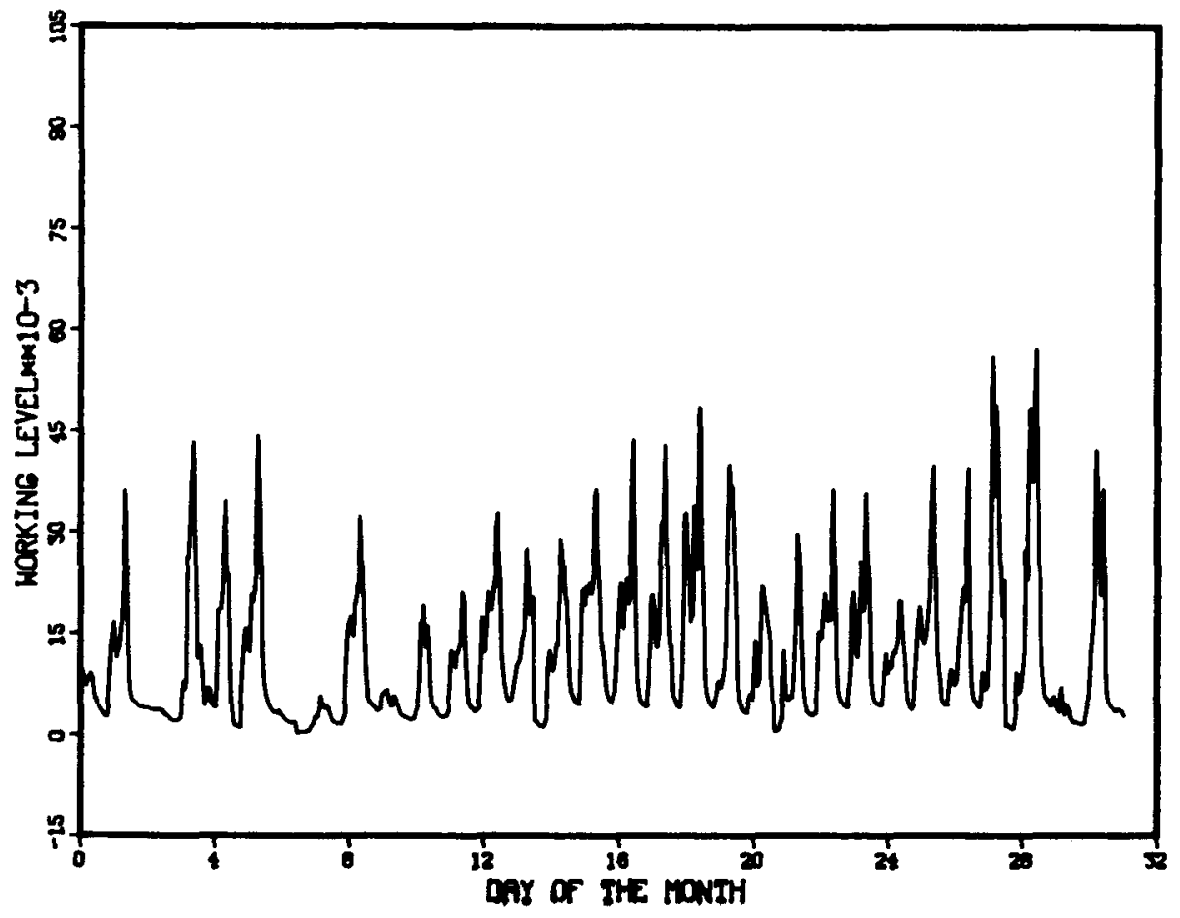

Figure A12. Continued. 
STATION 103, NOVEMBER 1977

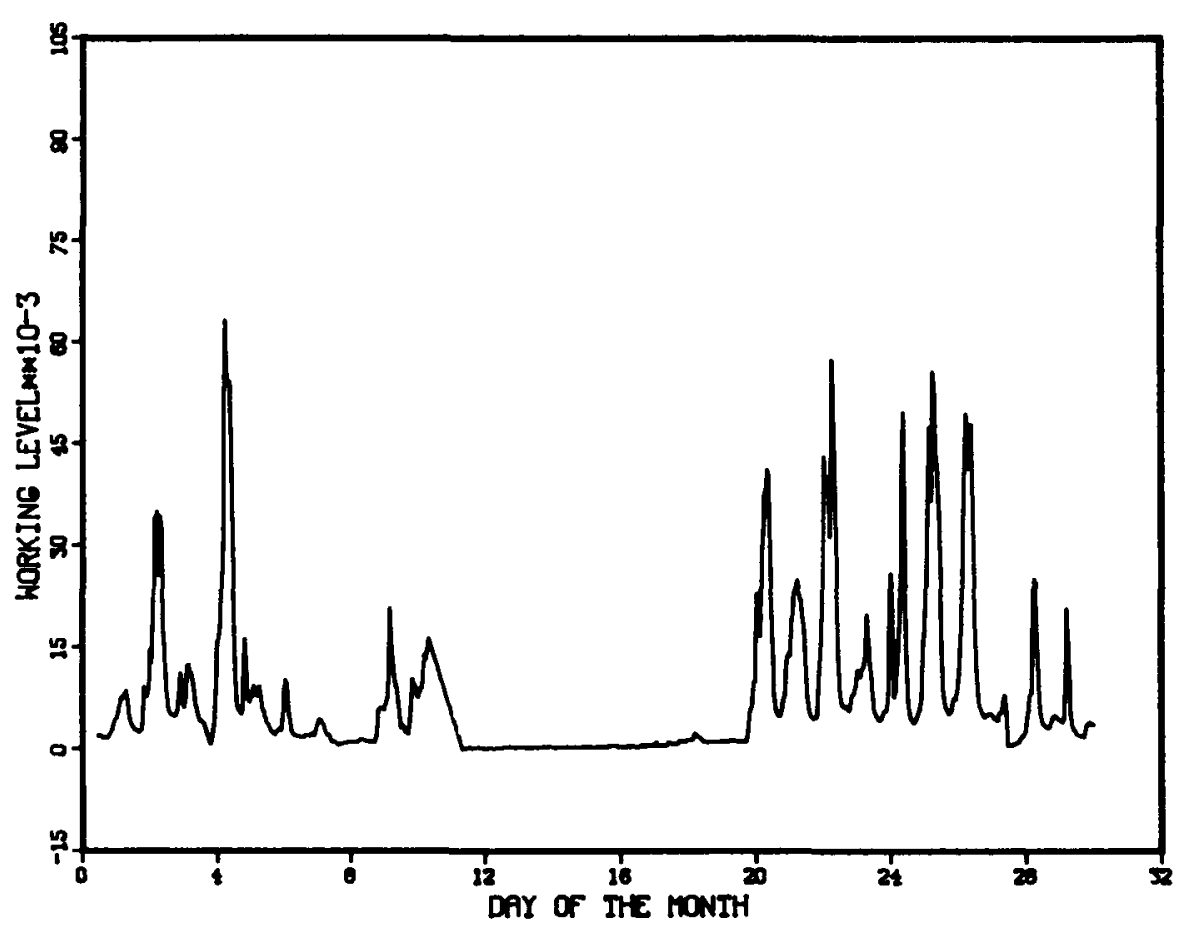

STATION 103, DECEMBER 1977

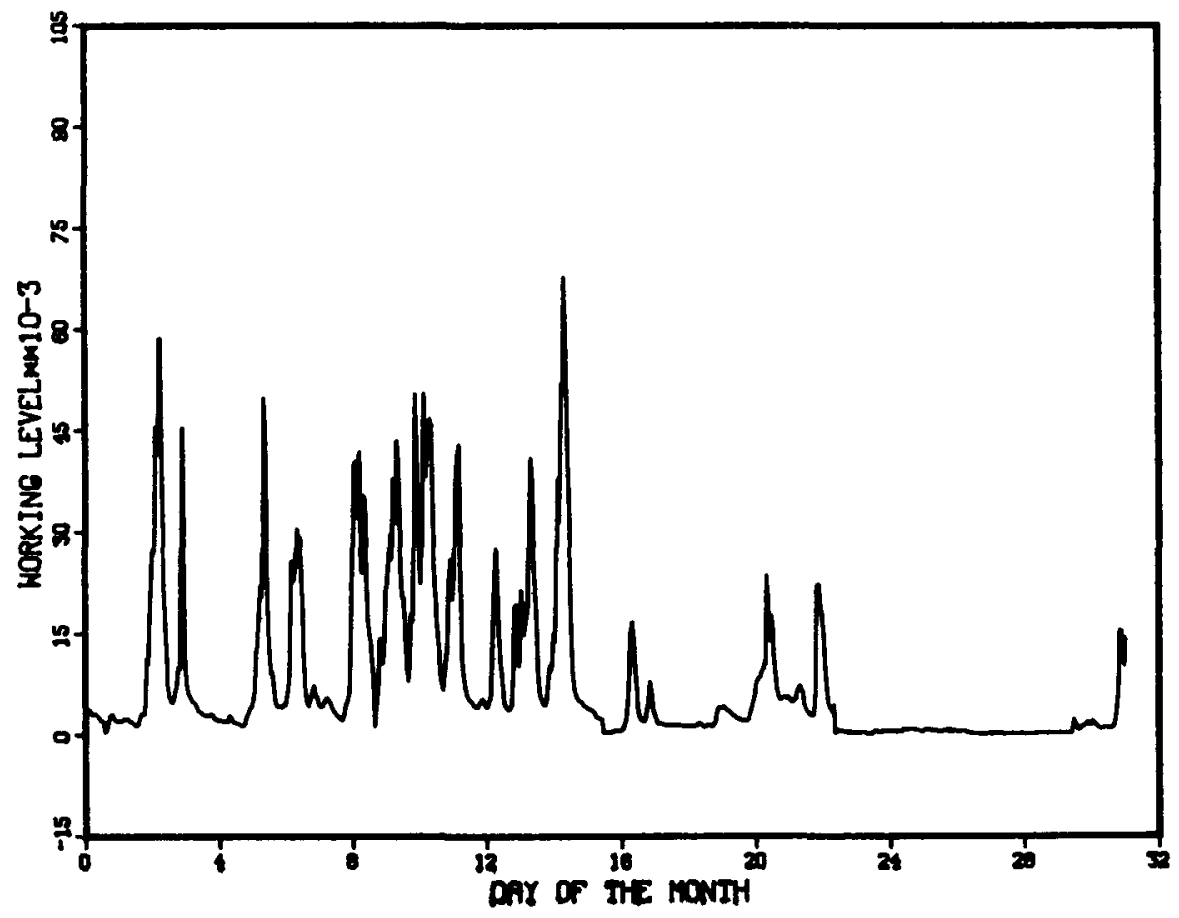

Figure Al2. Continued. 
STATION 103, MRRCH 1978

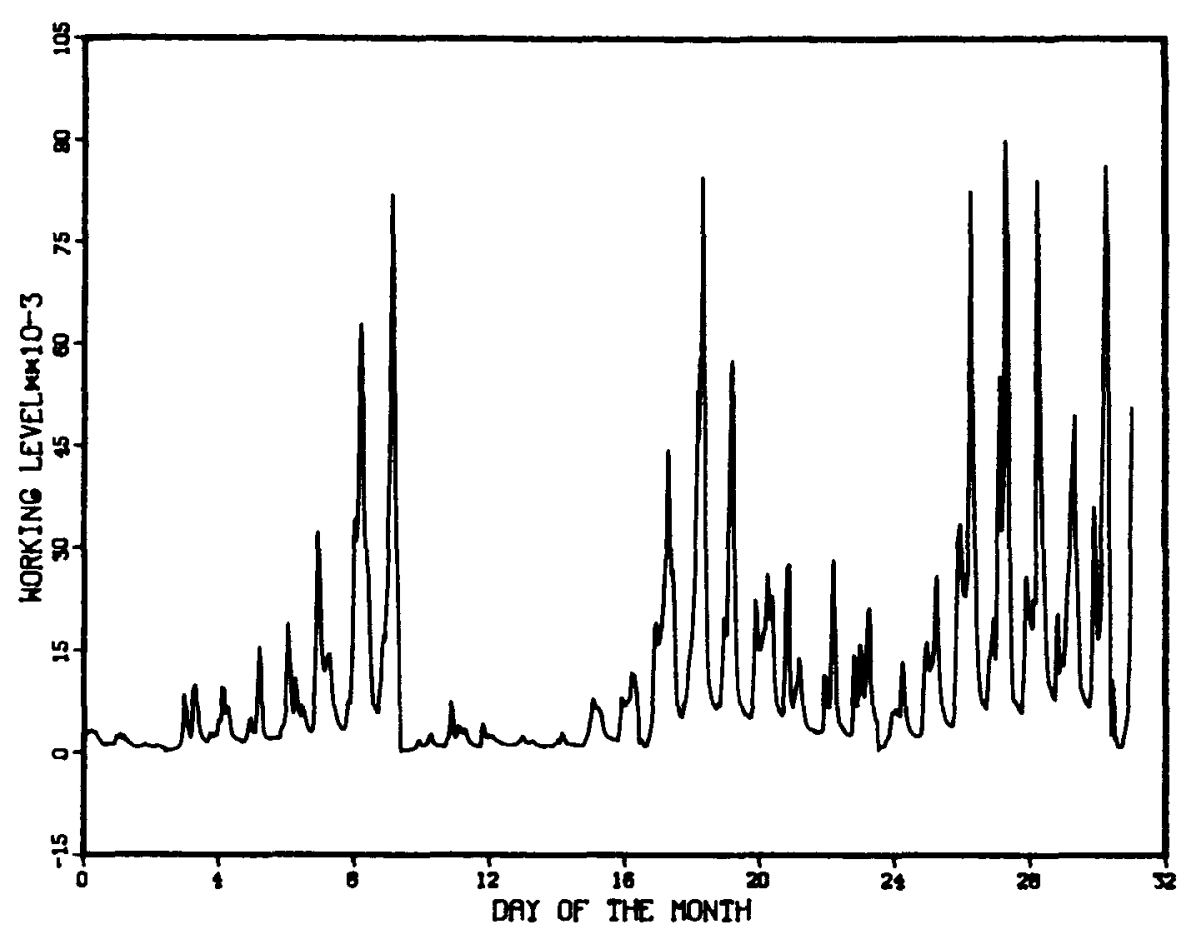

STATION 103, APRIL 1978

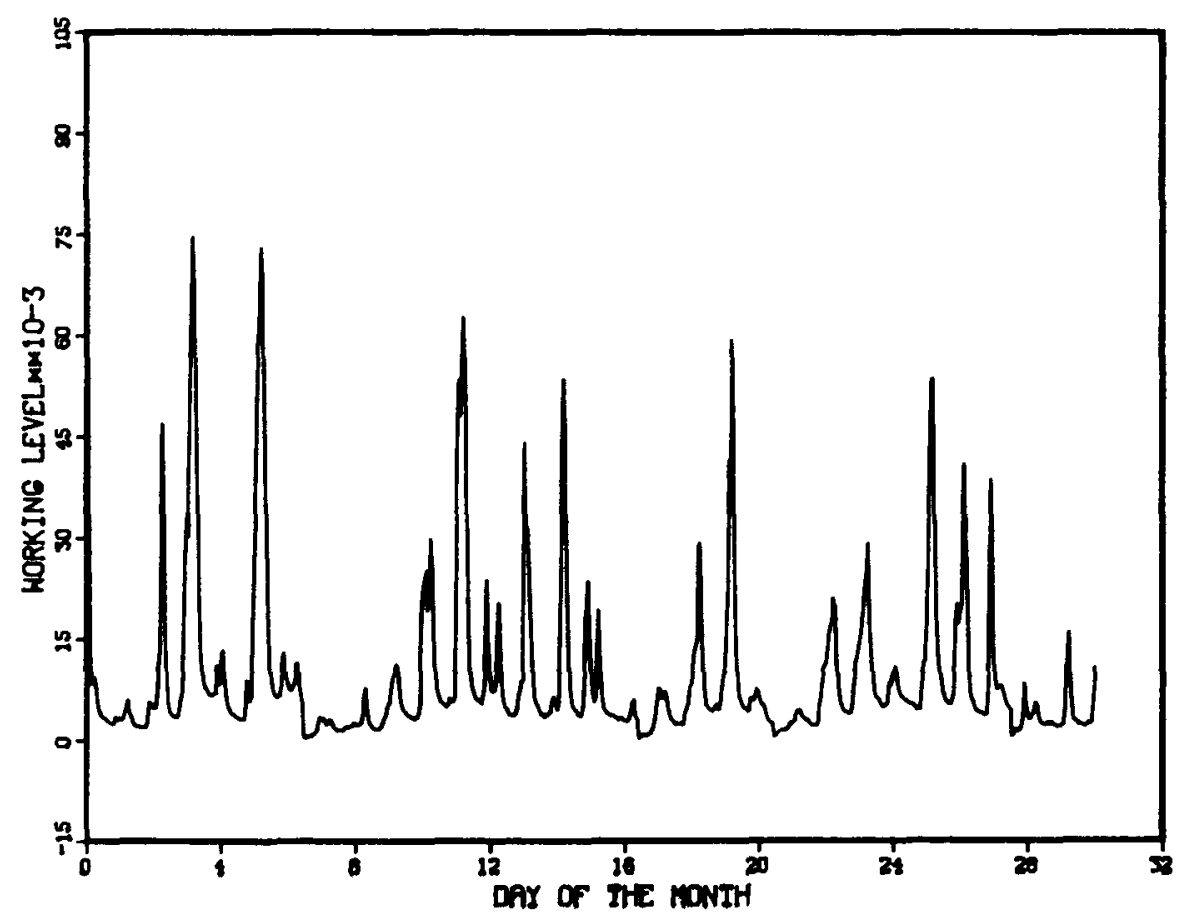

Figure Al2. Continued. 
STATION 103, MAY 1978

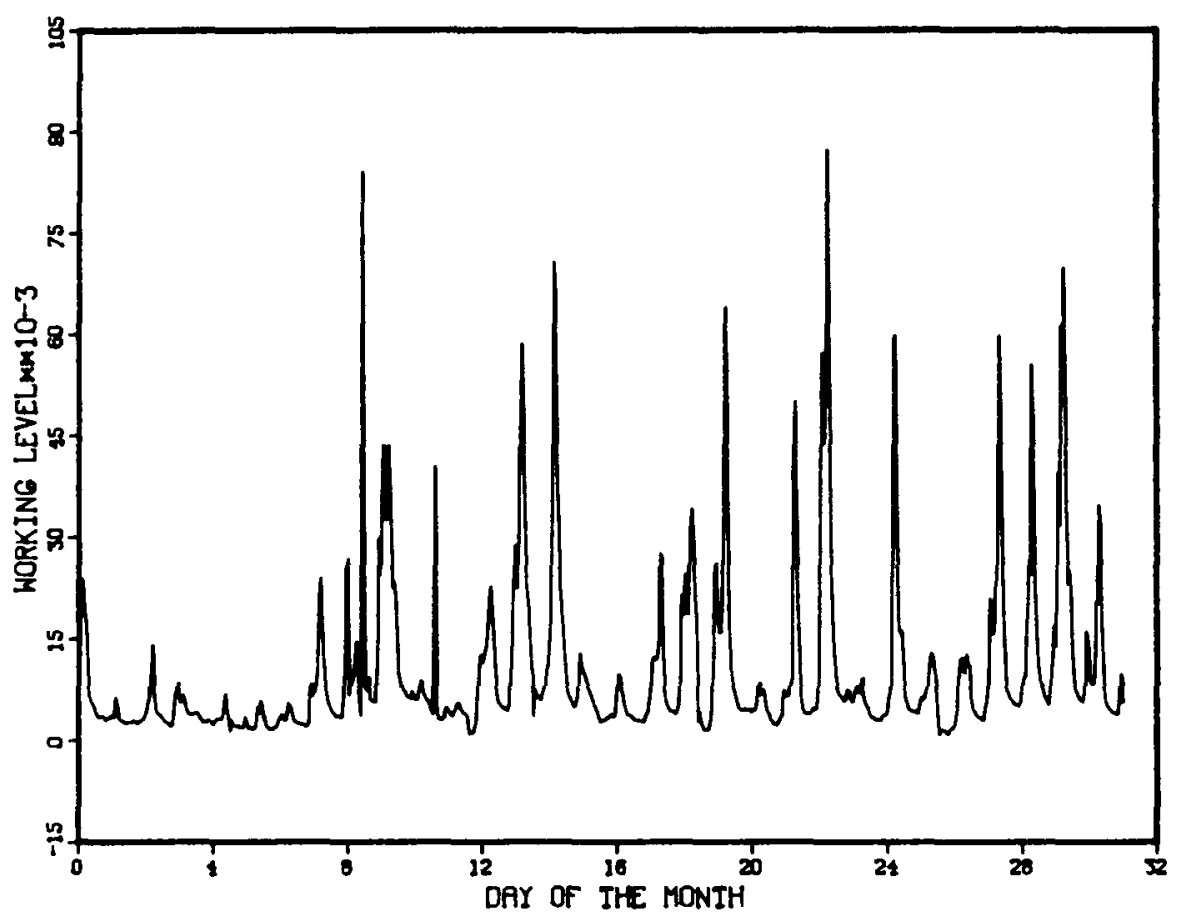

STATION 103, JUNE 1978

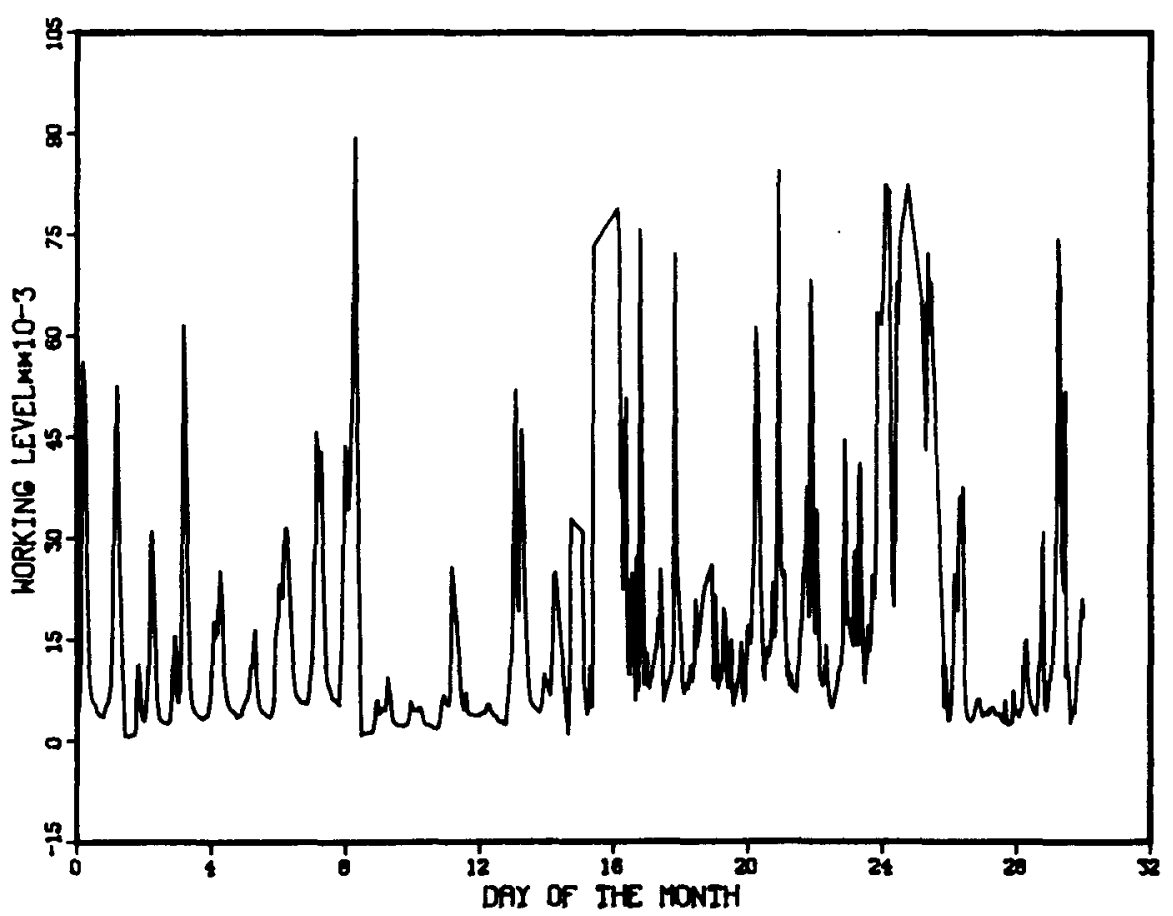

Figure A12. Continued. 
STATION 103, JULY 1978

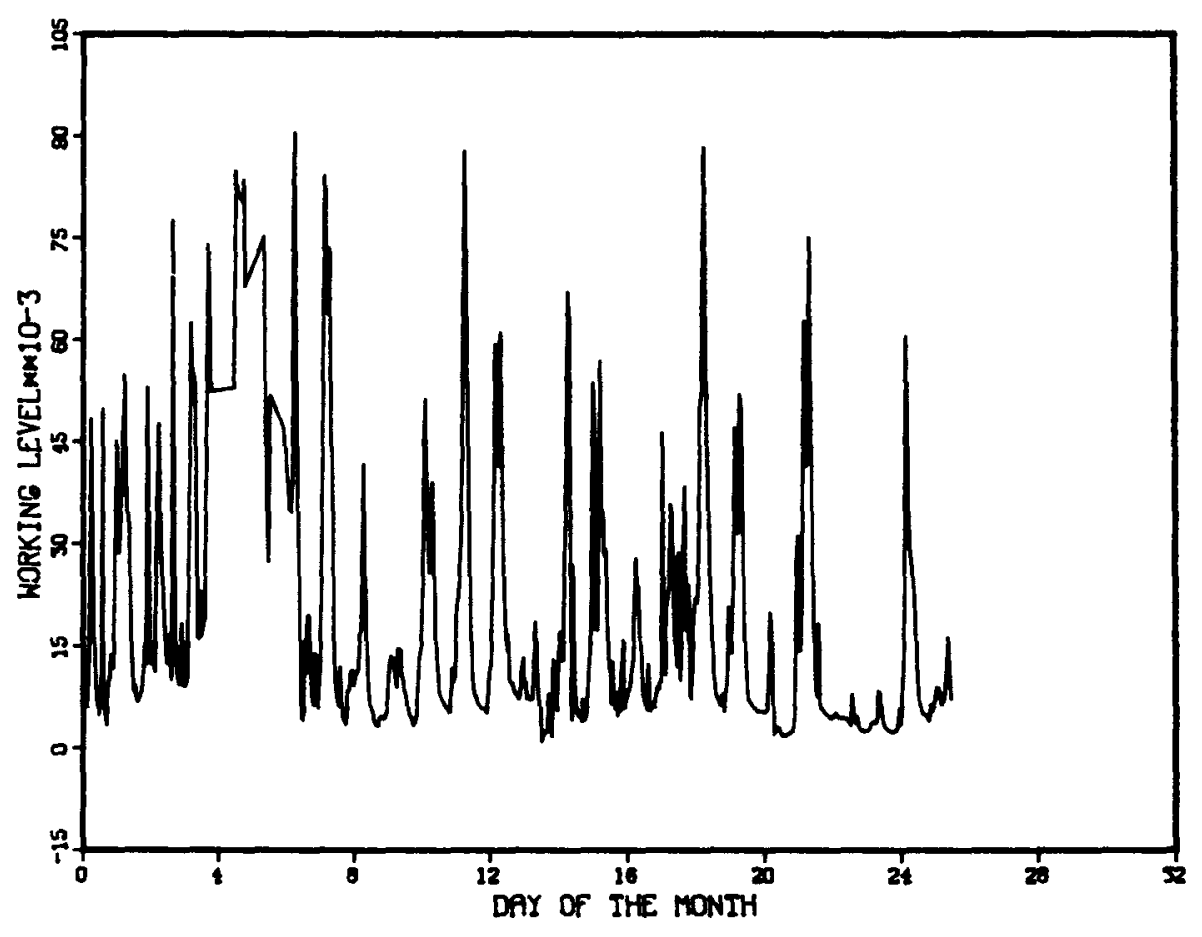

Figure Al2. Continued. 
STATION 104, JULY 1977

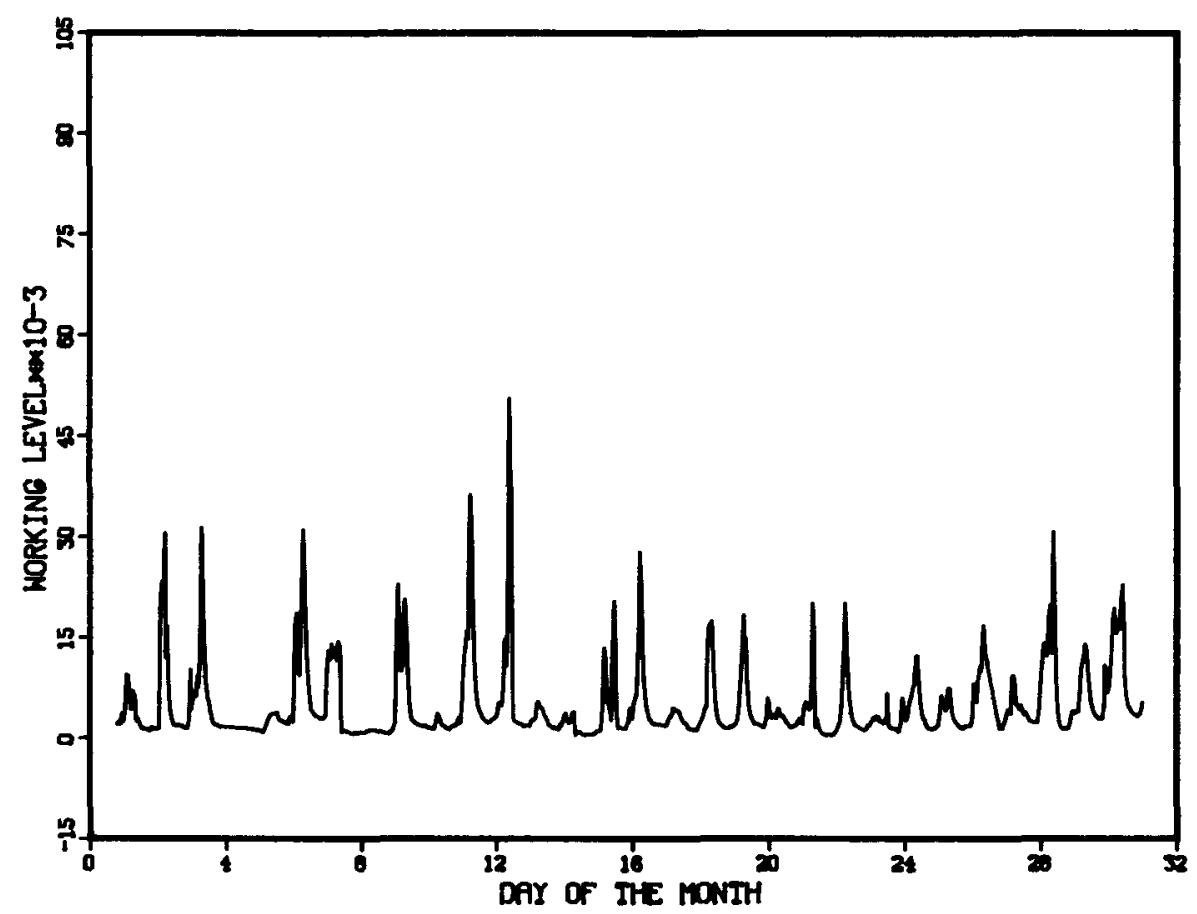

STATION 104, RUGUST 1977

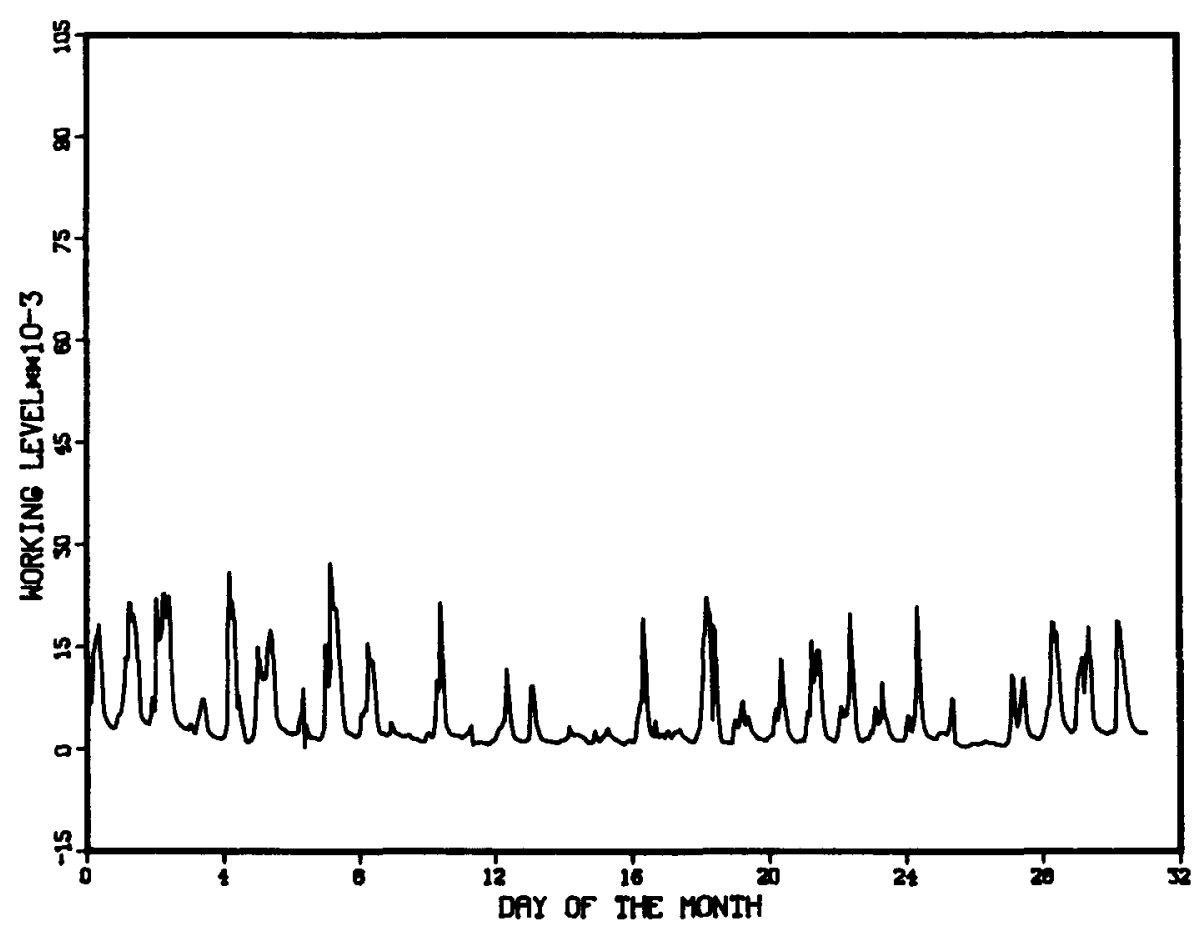

Figure A13. Working Level by Hour at Station 104 . 
STATION 104, SEPTEMBER 1977

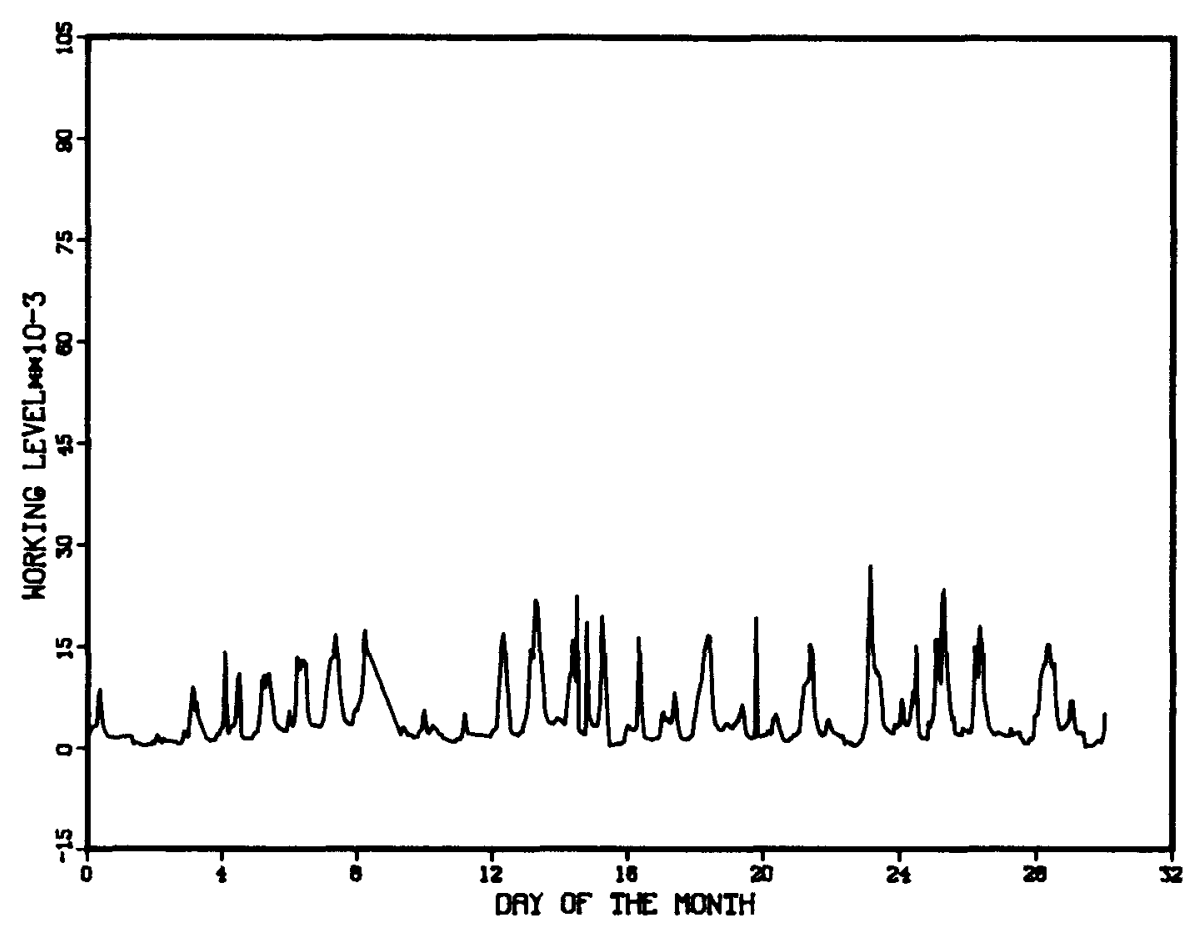

STATION 104, OCTOBER 1977

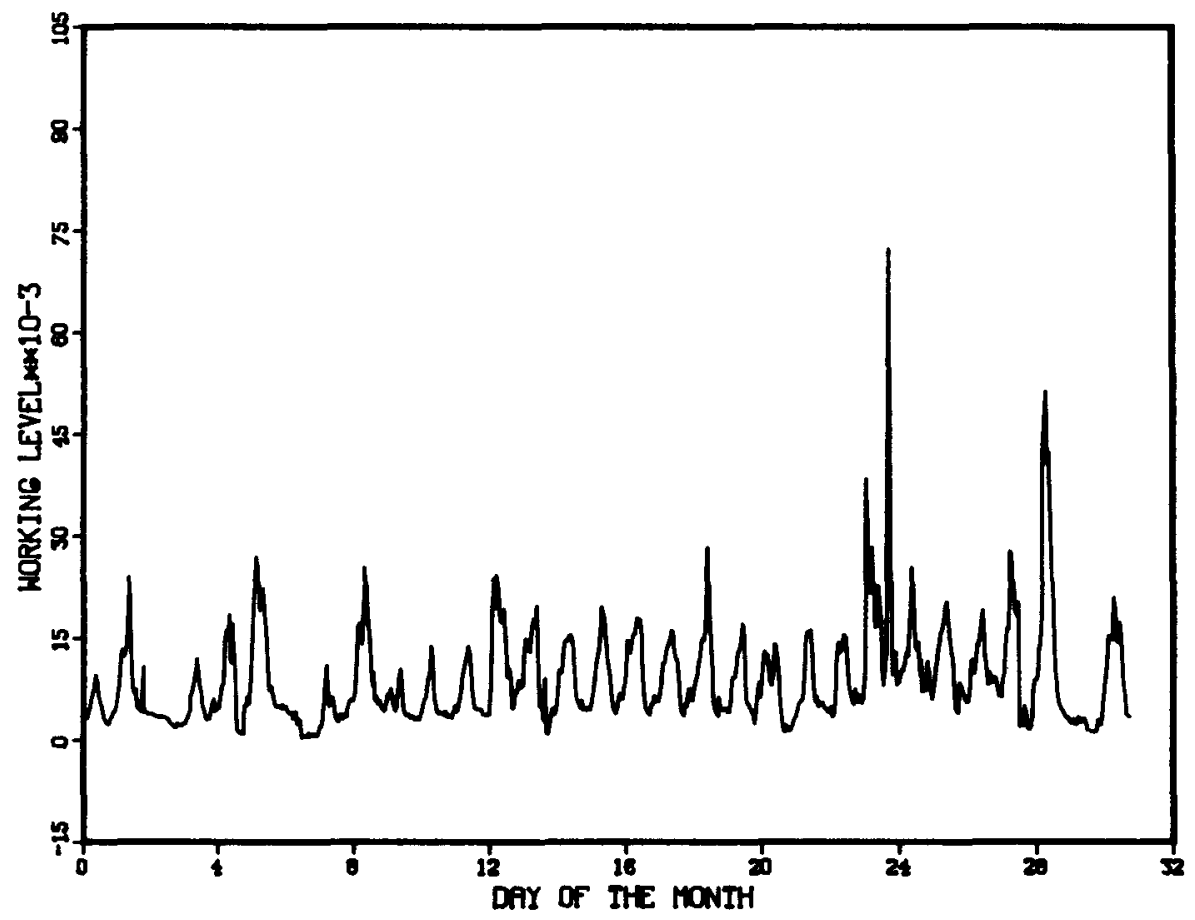

Figure Al3. Continued. 
STATION 104, FEBRURRY 1978

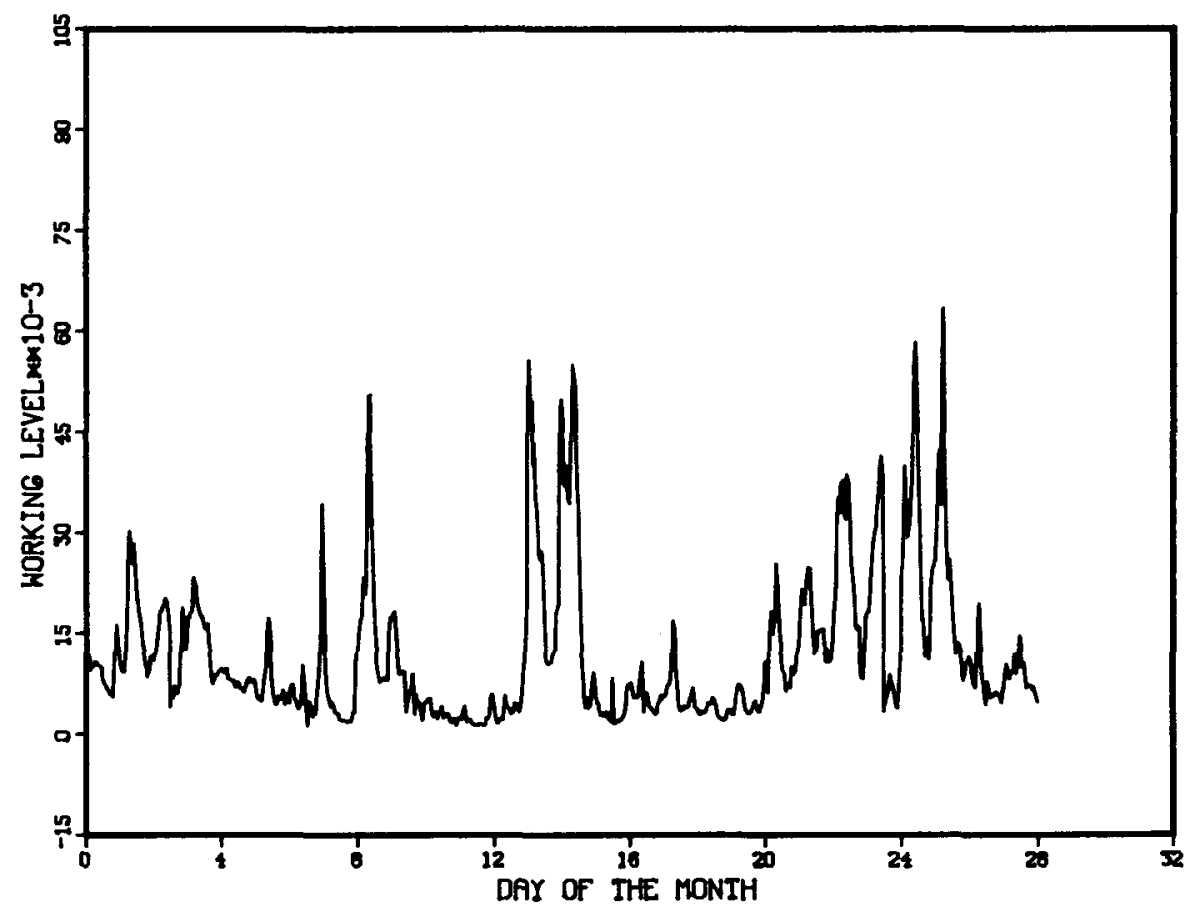

STATION 104, MARCH 1978

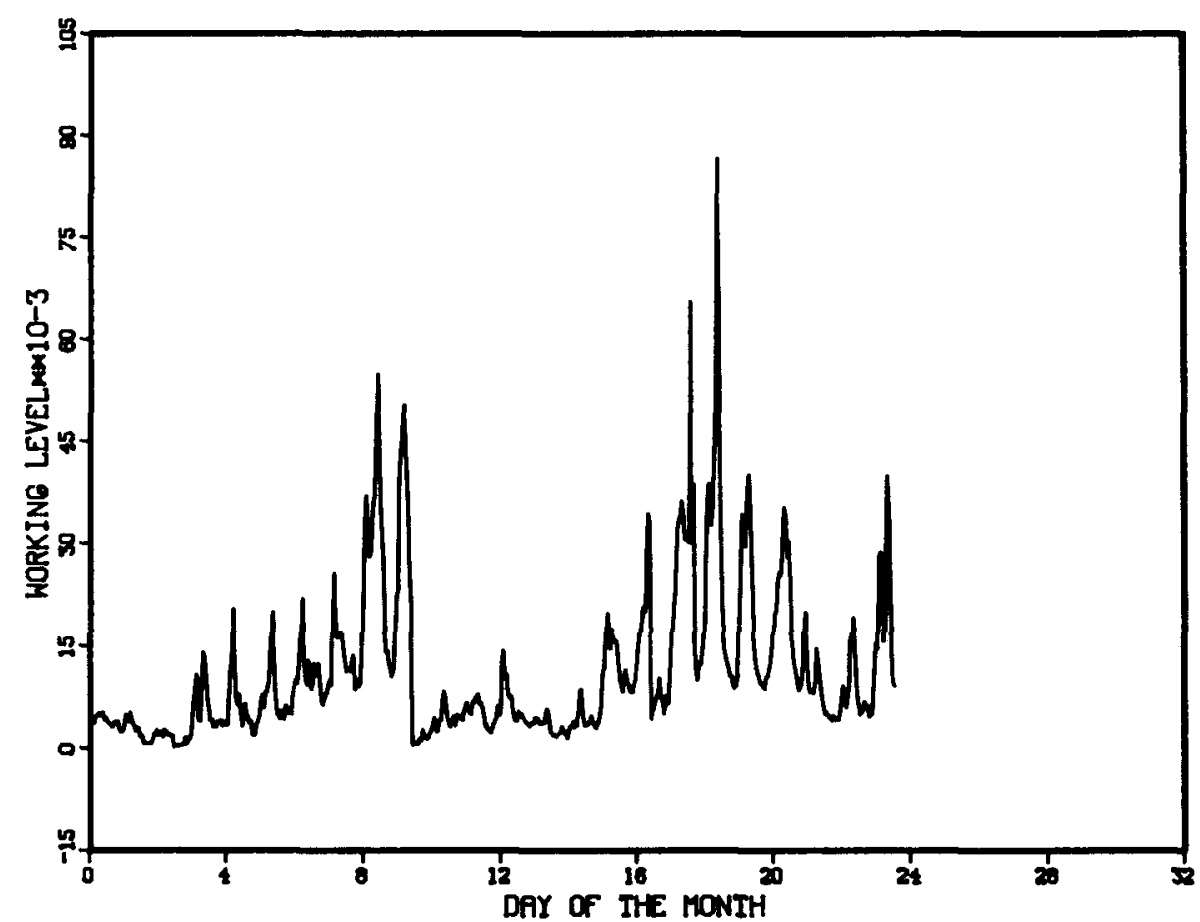

Figure Al3. Continued. 
STATION 102, JULY 1977

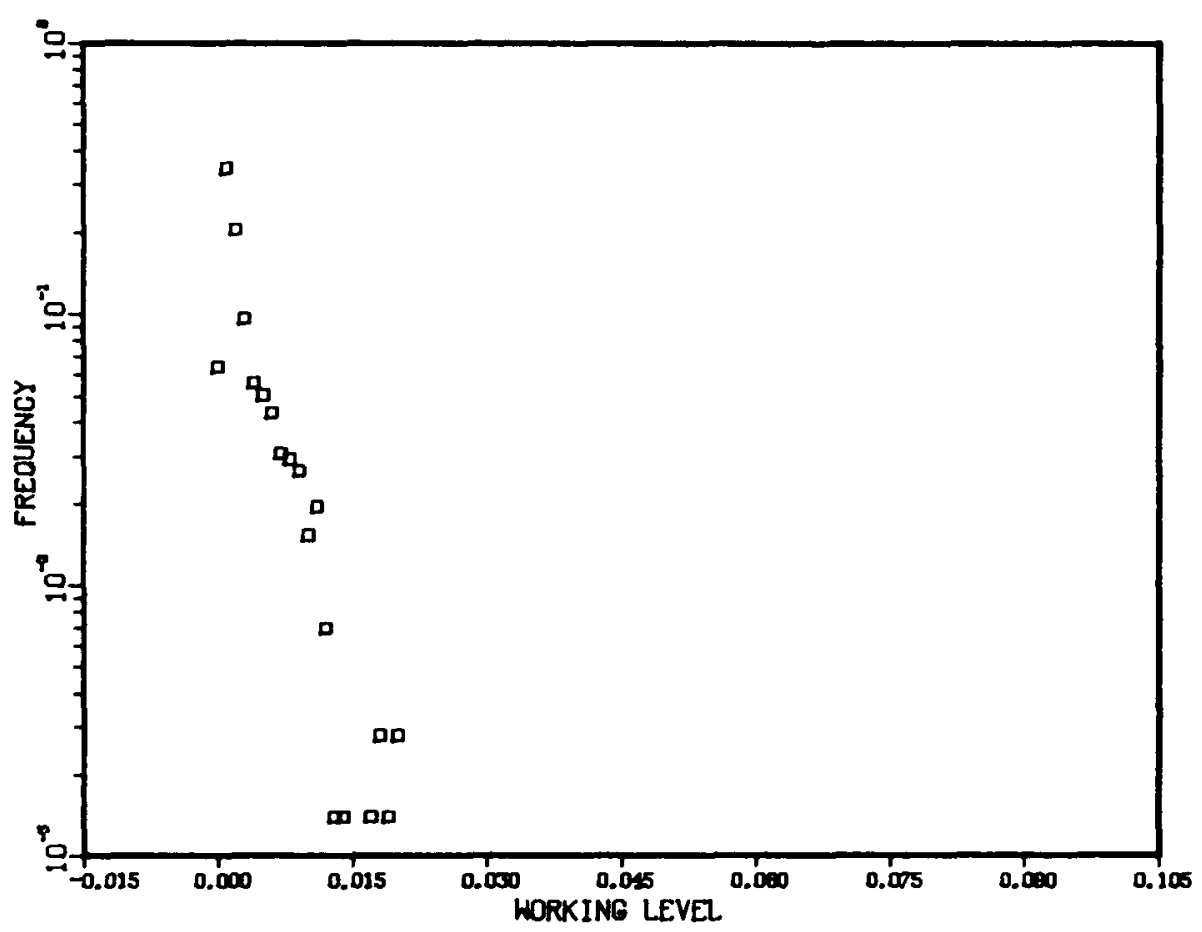

STATION 102, AUGUST 1977

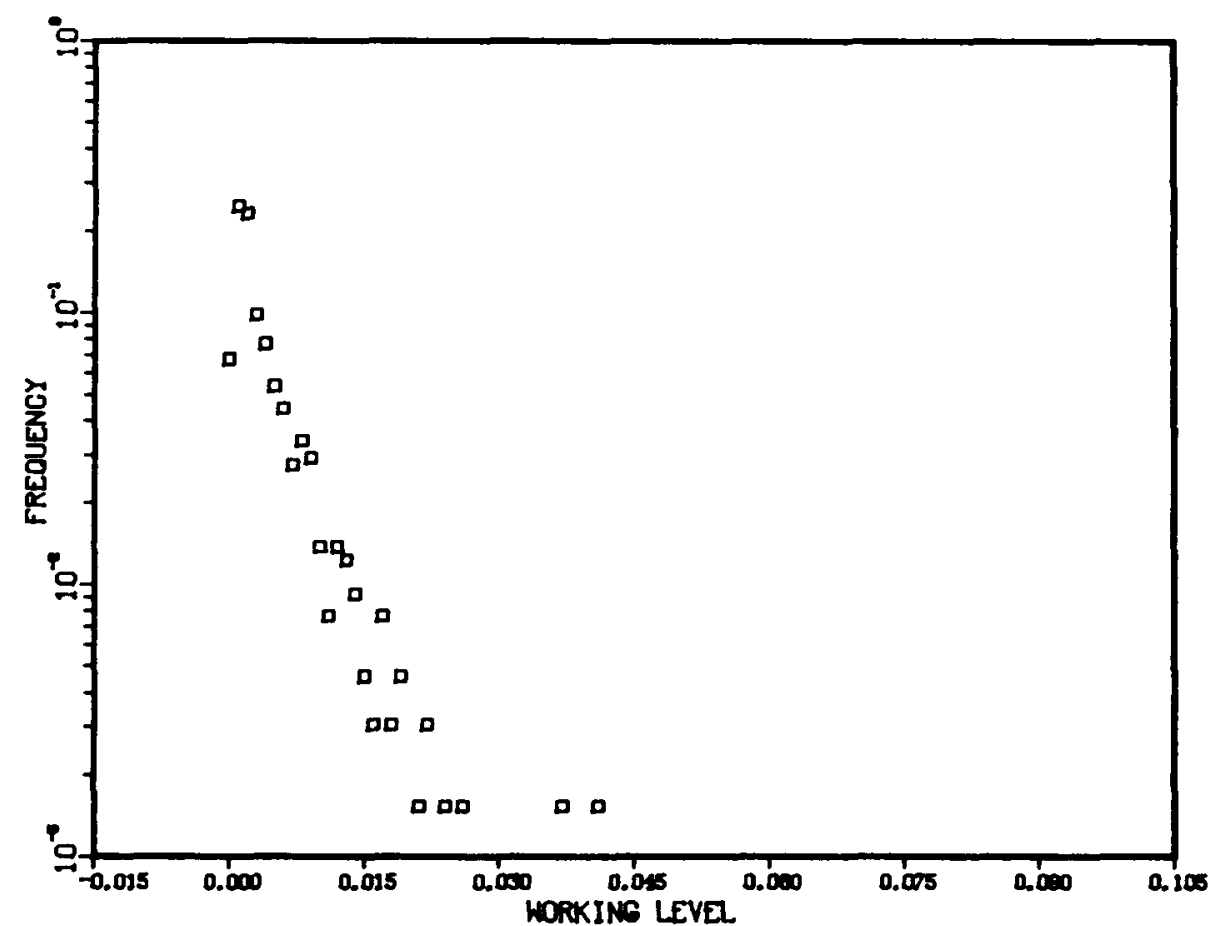

Figure A14. Working-Level Frequency at Station 102. 
STATION 102, SEPTEMBER 1977

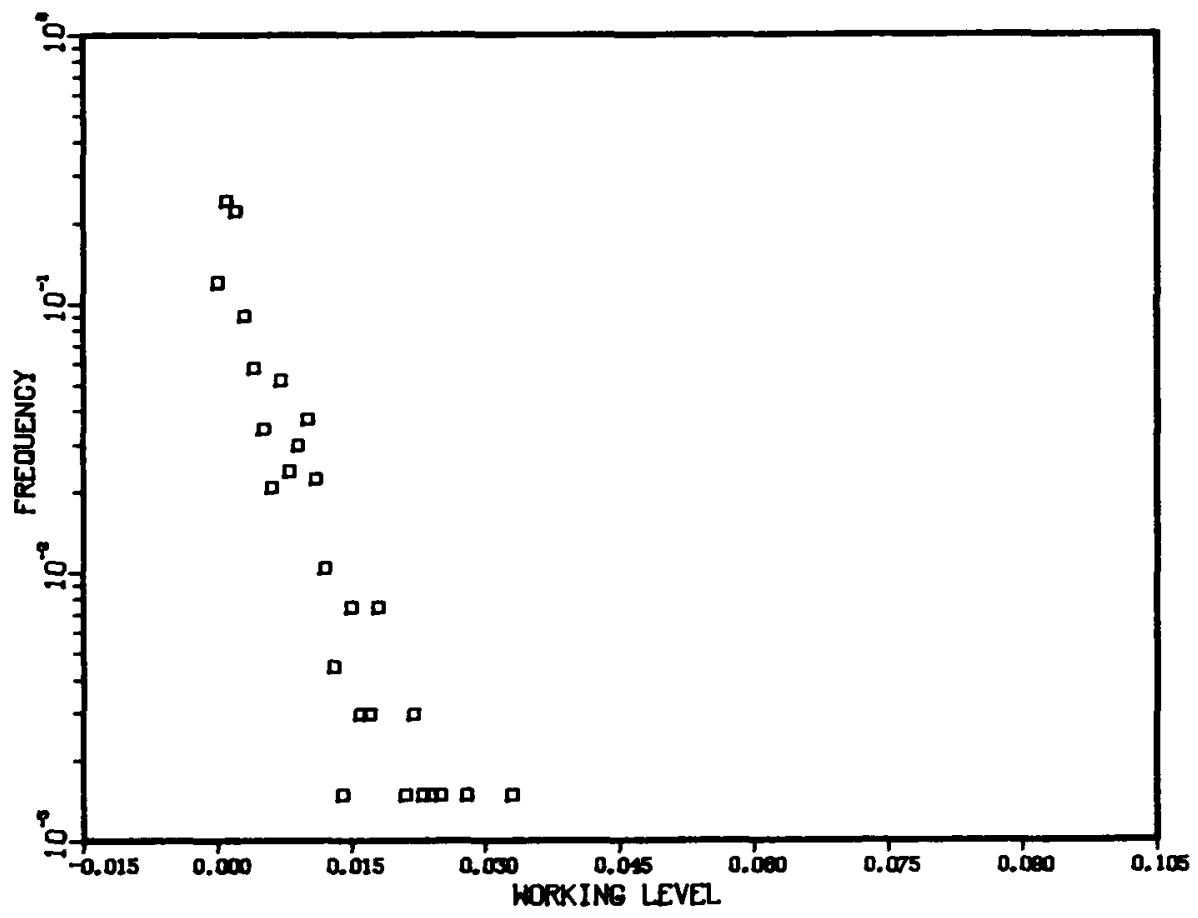

STATION 102, OCTOBER 1977

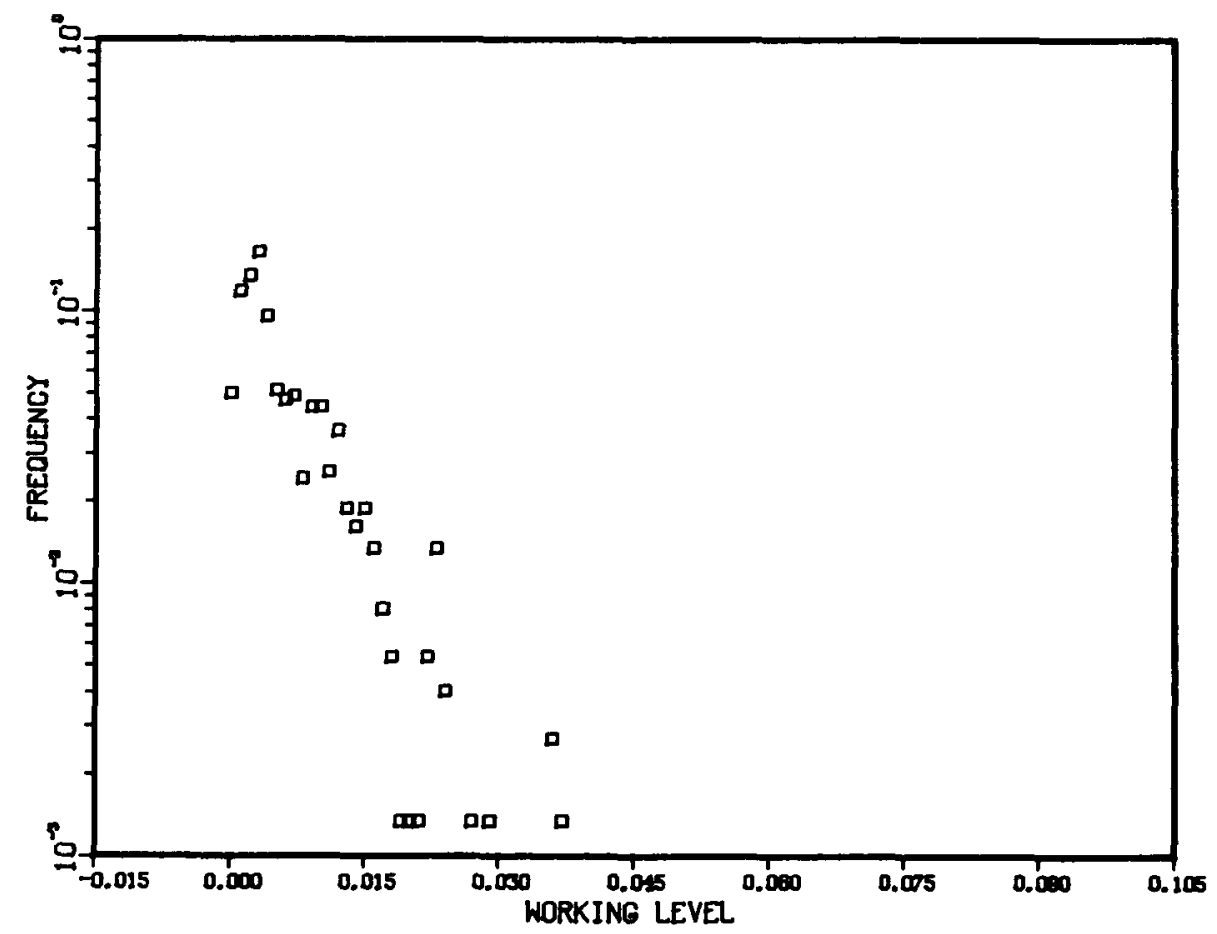

Figure Al4. Continued. 

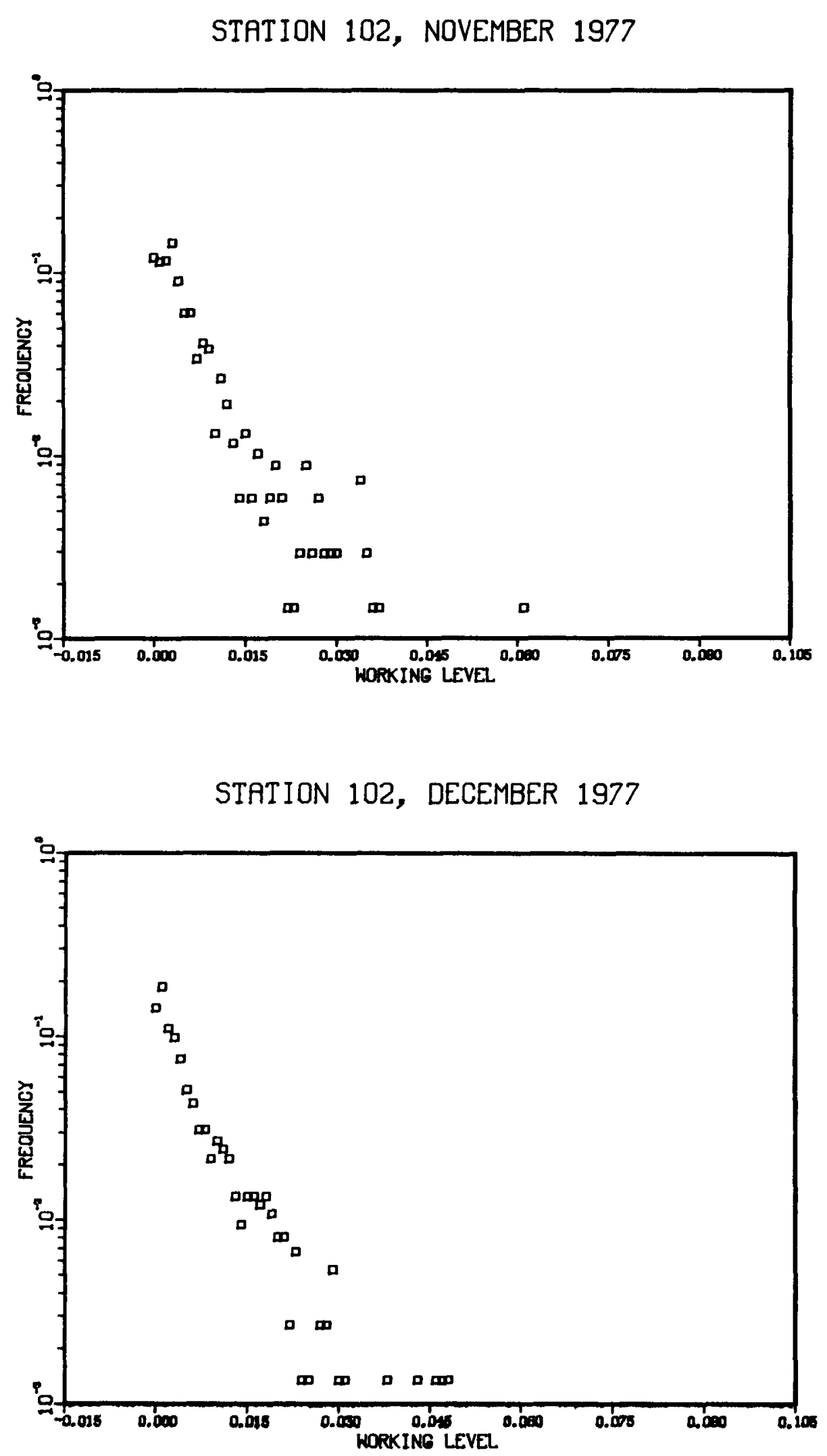

Figure Al4. Continued. 
STATION 102, MARCH 1978

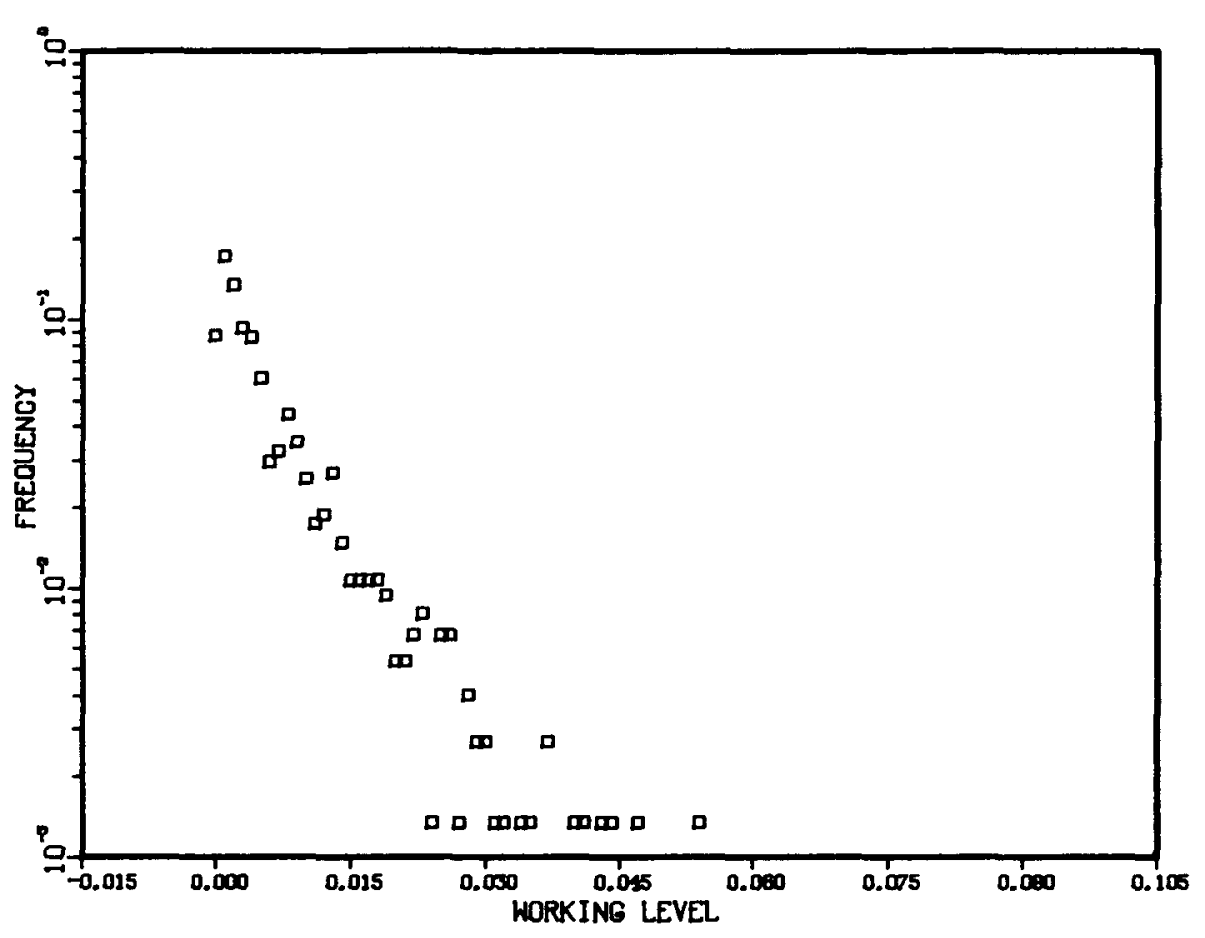

STATION 102, APRIL 1978

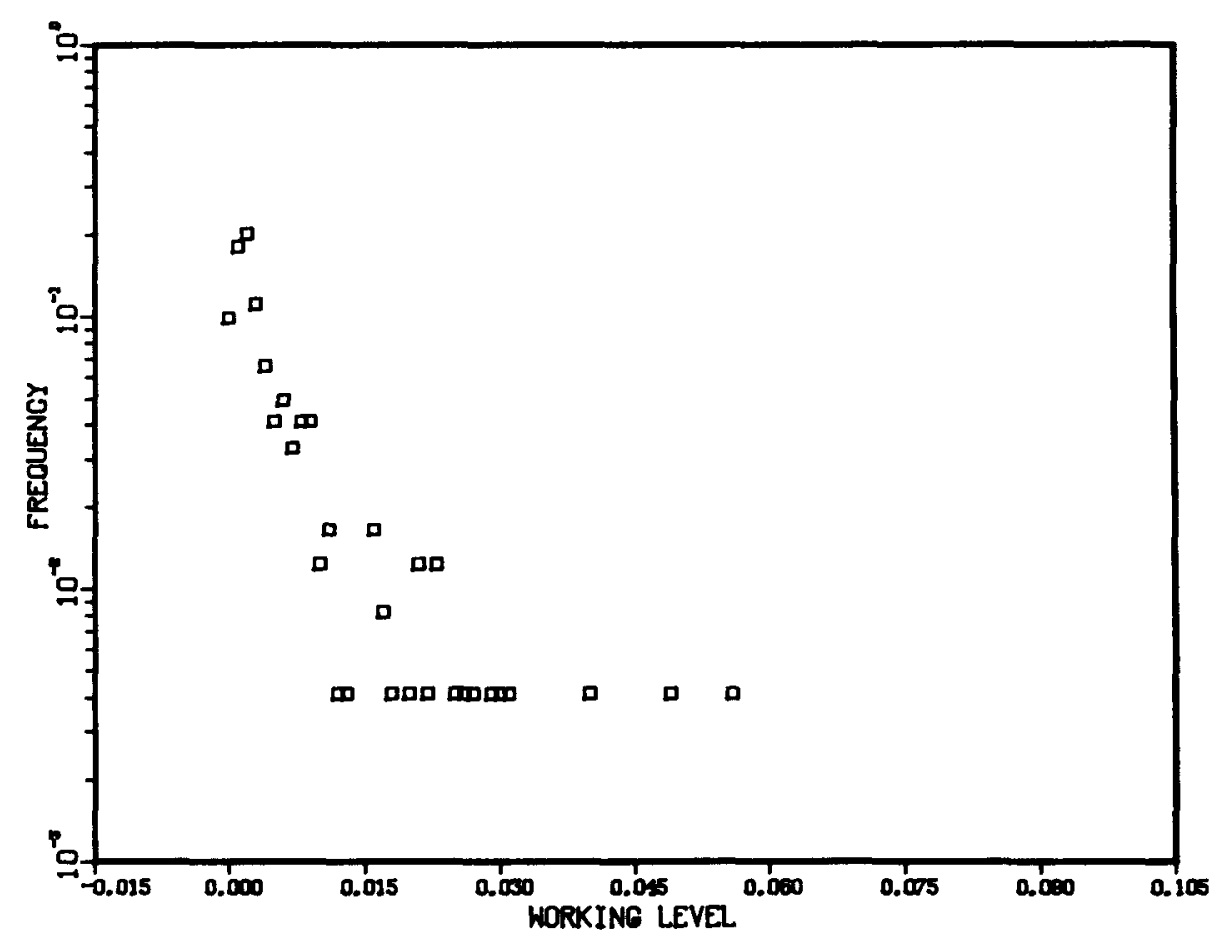

Figure Al4. Continued. 
STATION 102, JUNE 1978

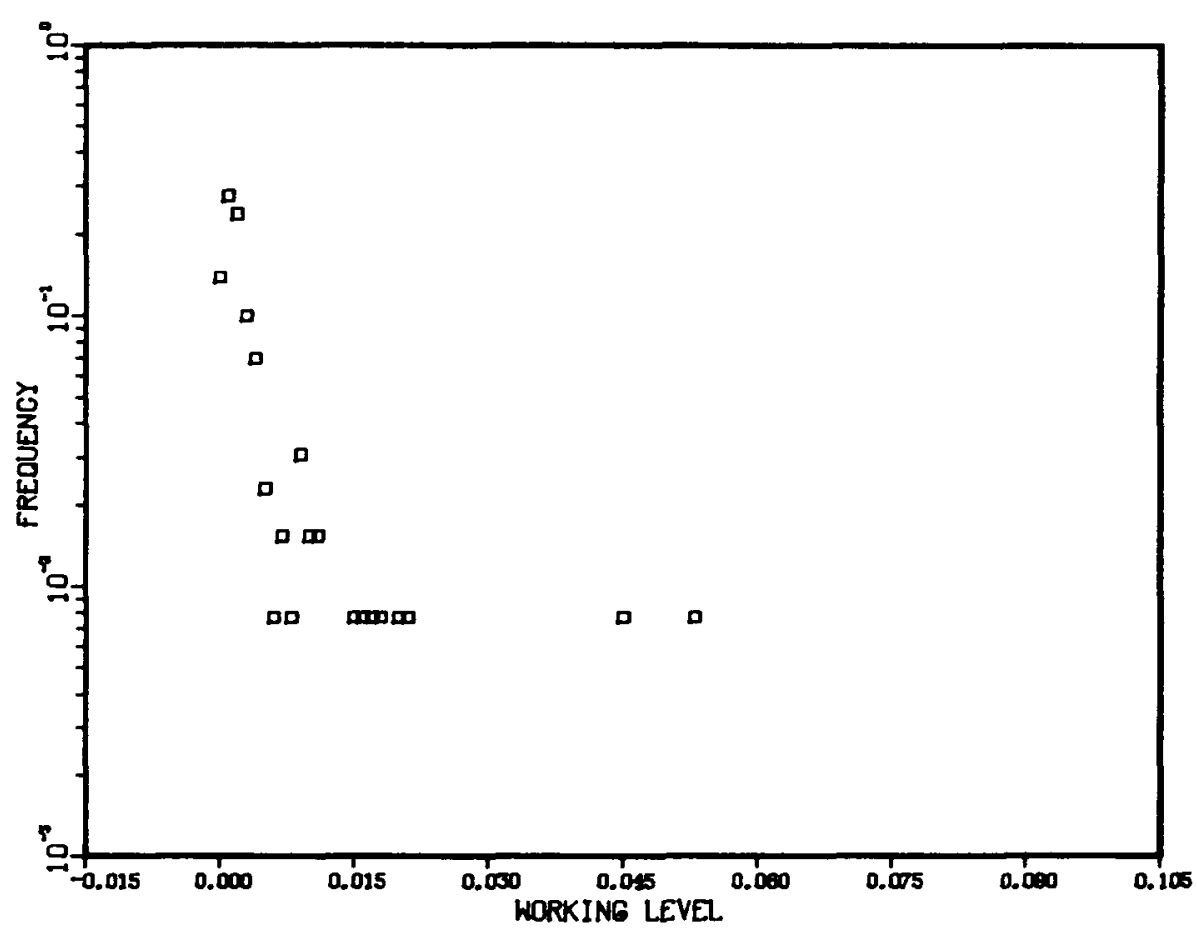

Figure Al4. Continued. 


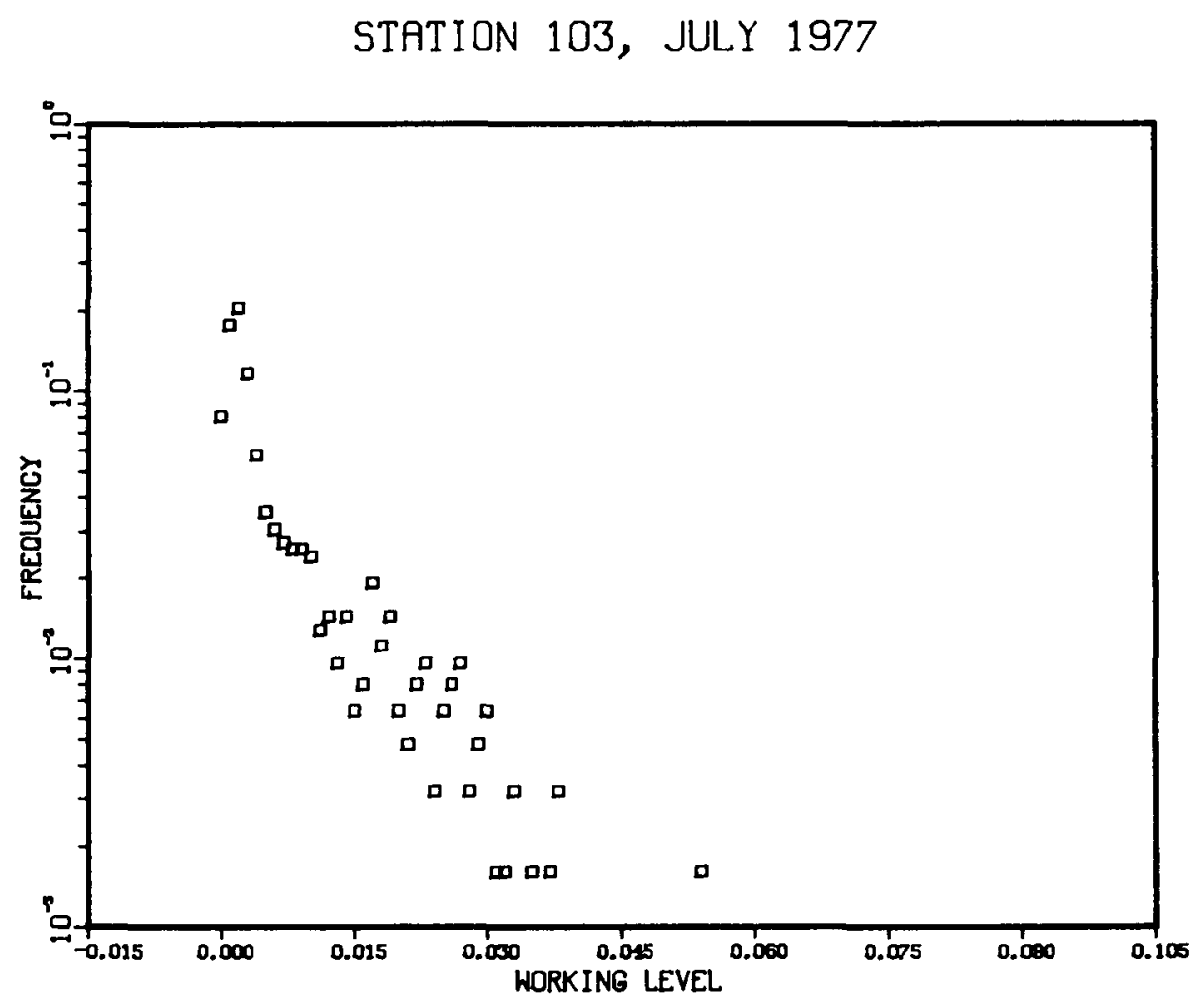

STATION 103, AUGUST 1977

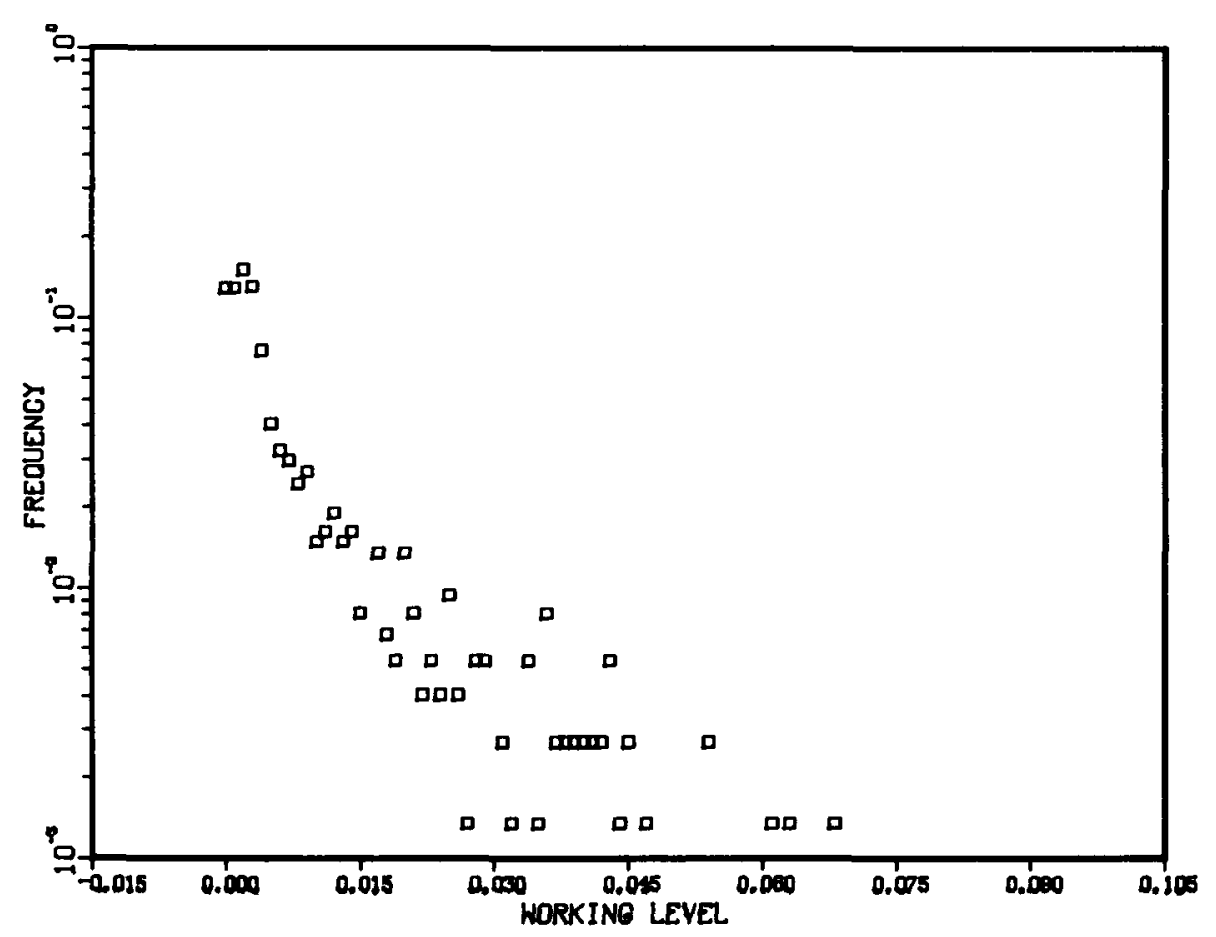

Figure Al5. Working-Level Frequency at Station 103. 
STATION 103, SEPTEMBER 1977

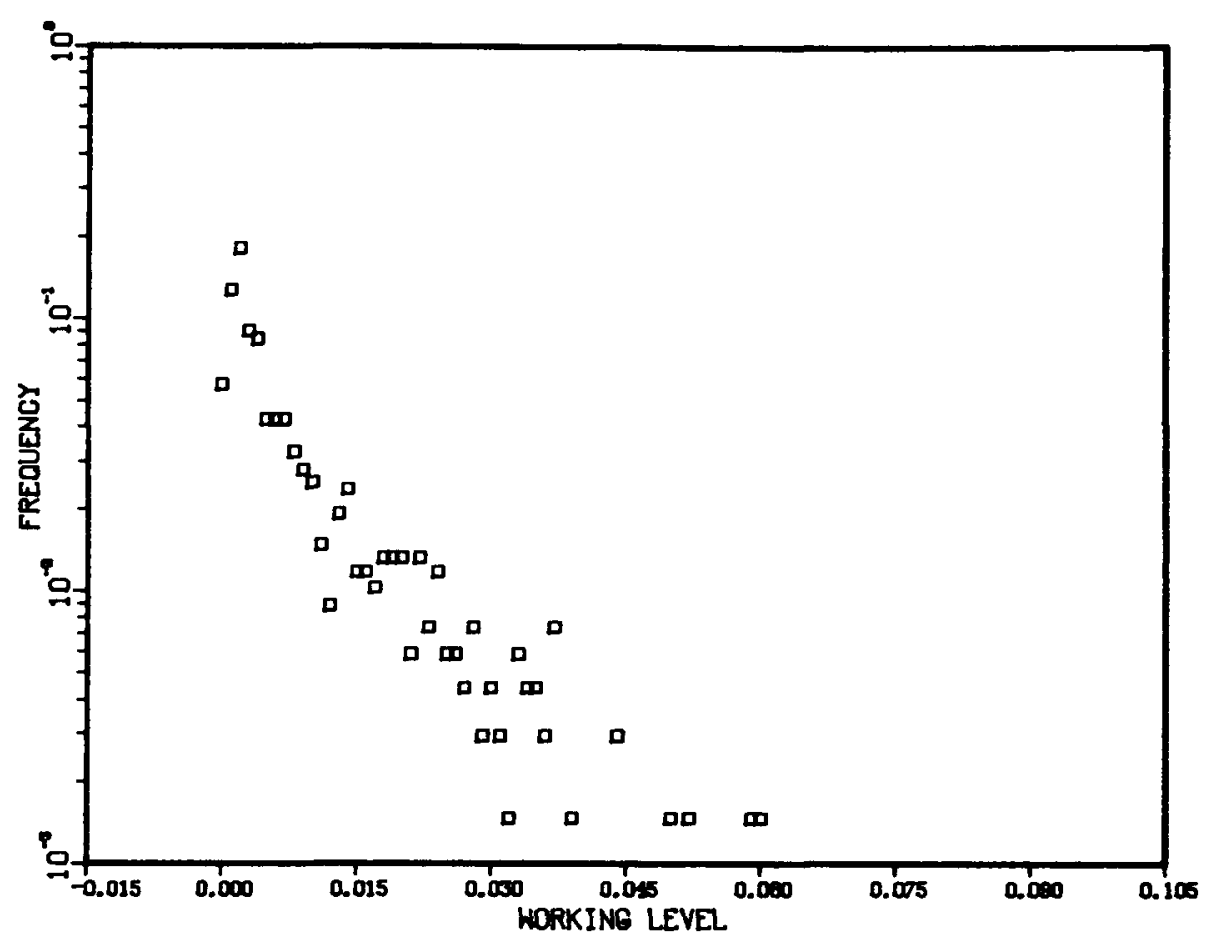

STATION 103, OCTOBER 1977

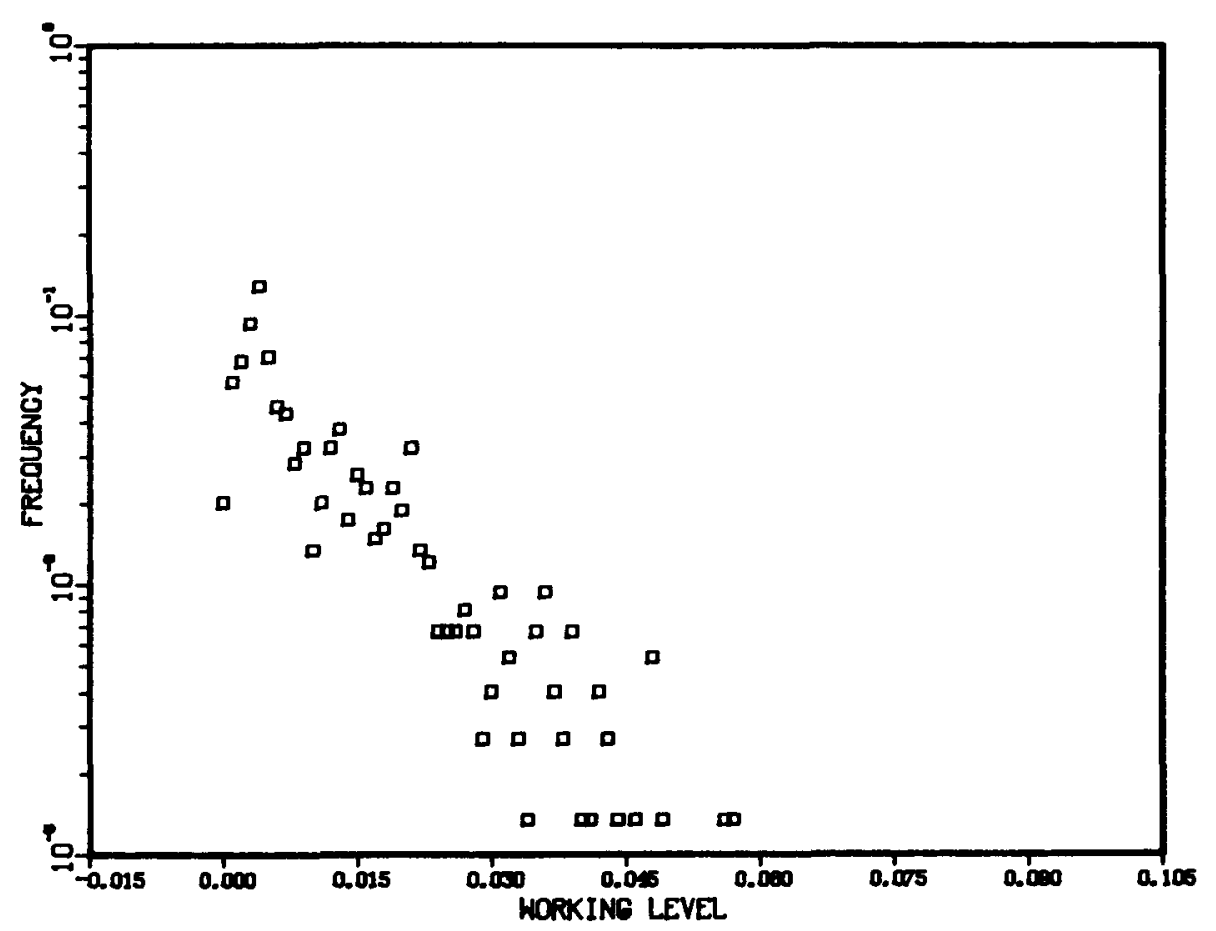

Figure Al5. Continued. 
STATION 103, NOVEMBER 1977

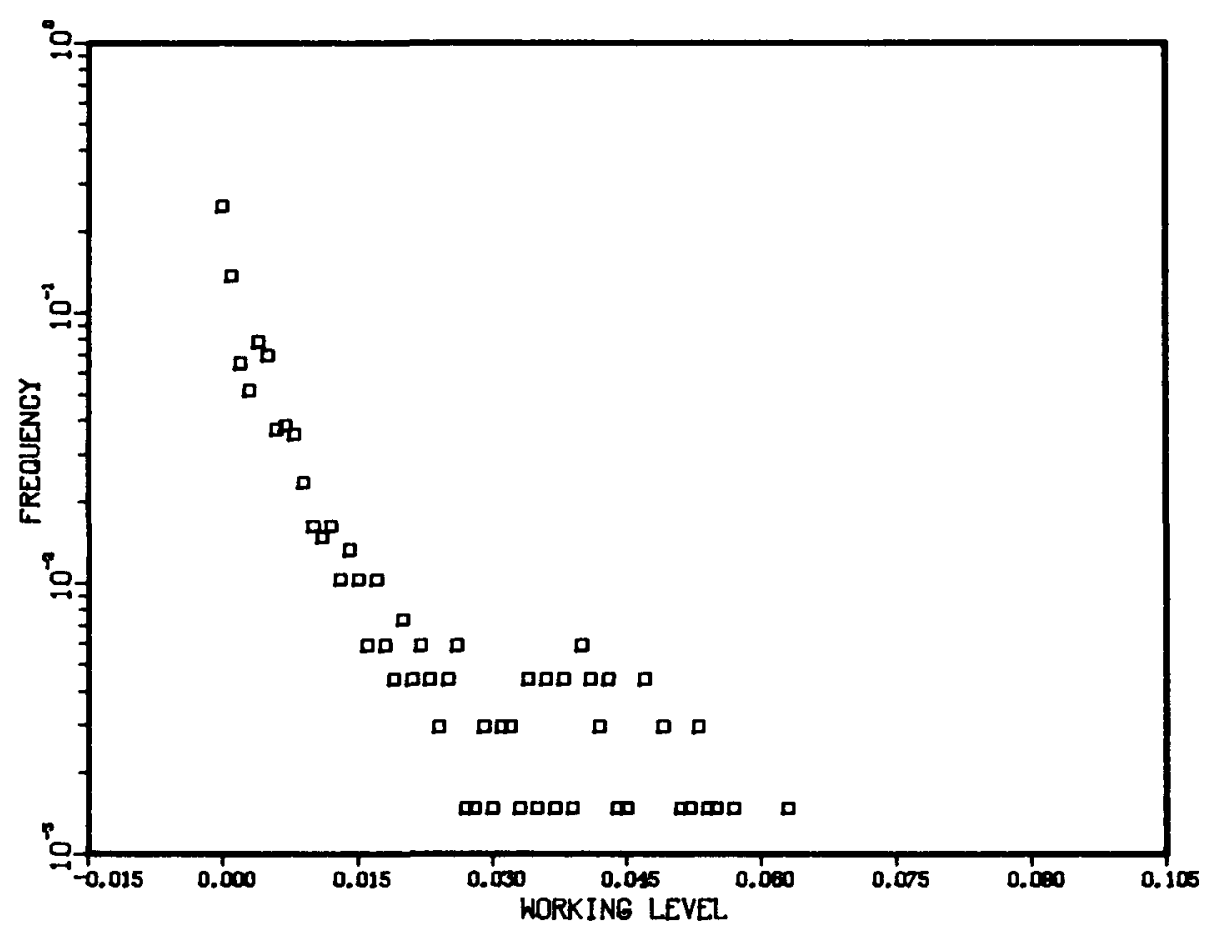

STATION 103, DECEMBER 1977

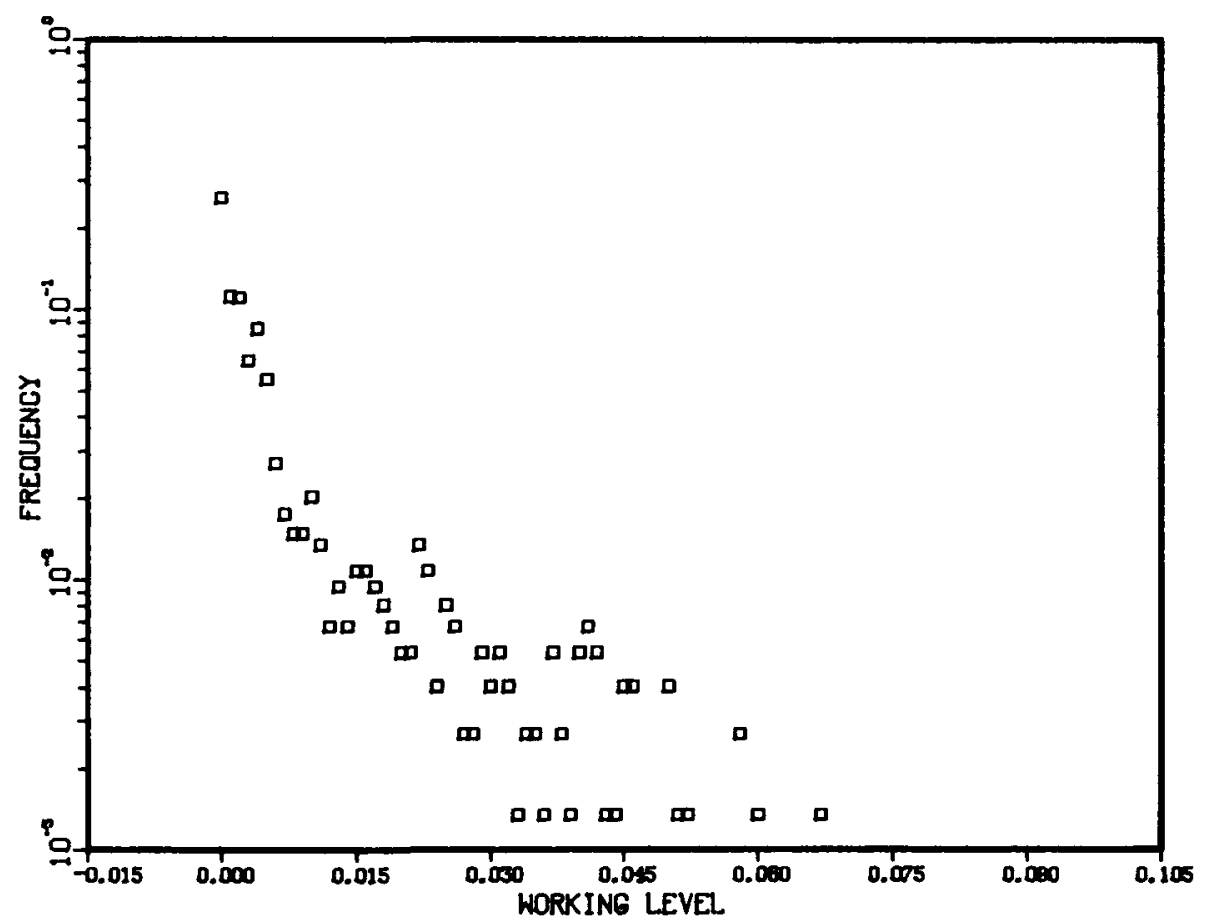

Figure A15. Continued. 
STATION 103, MARCH 1978

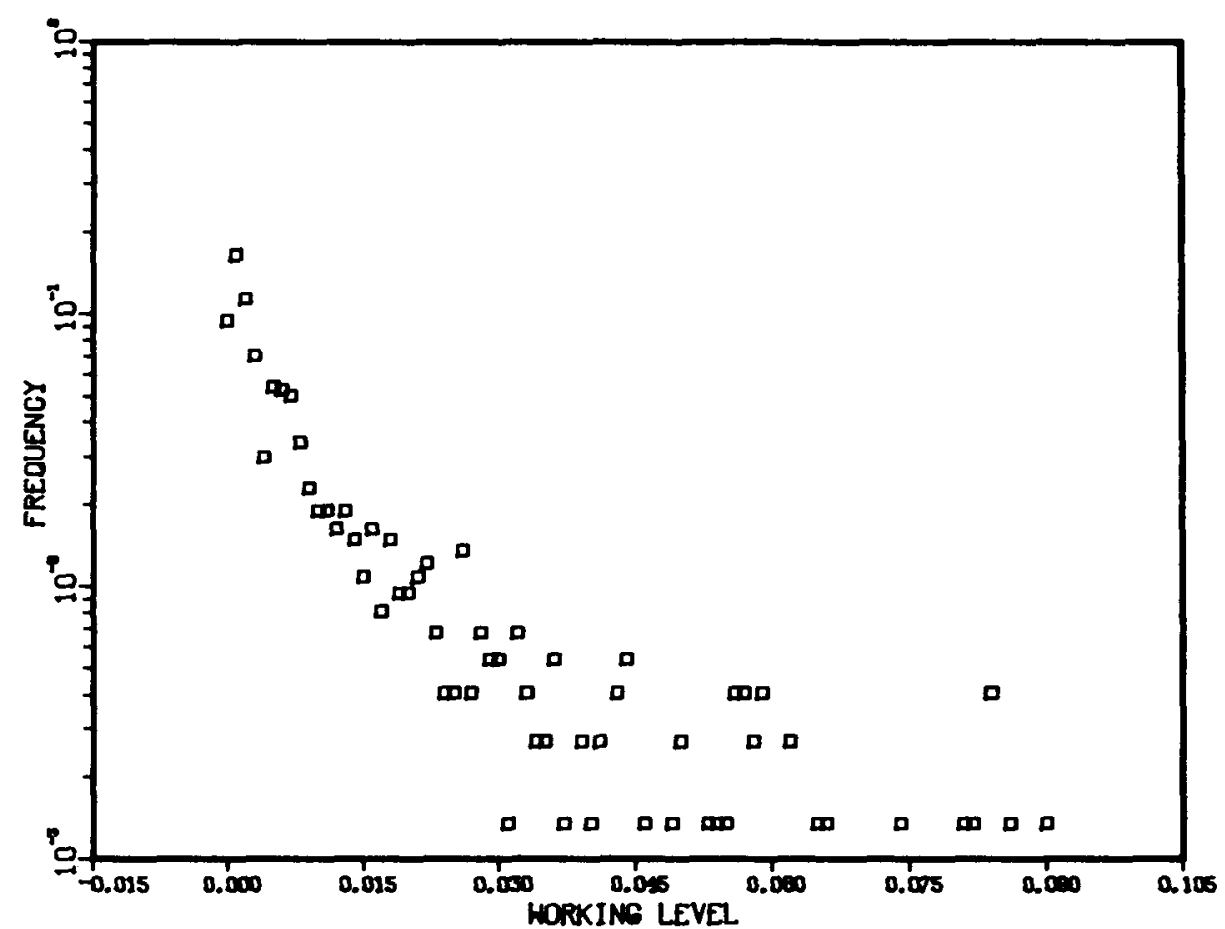

STATION 103, APRIL 1978

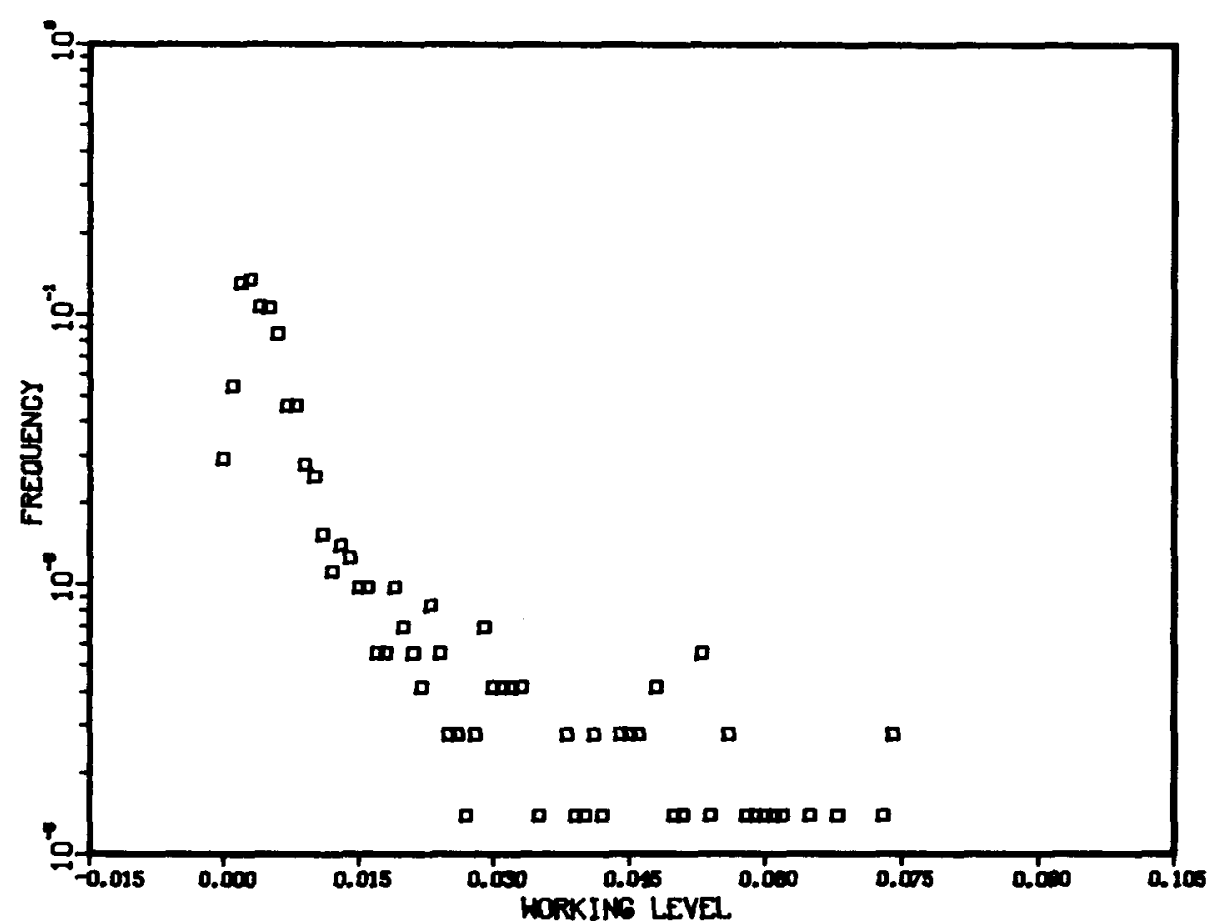

Figure A15. Continued. 
STATION 103, MAY 1978

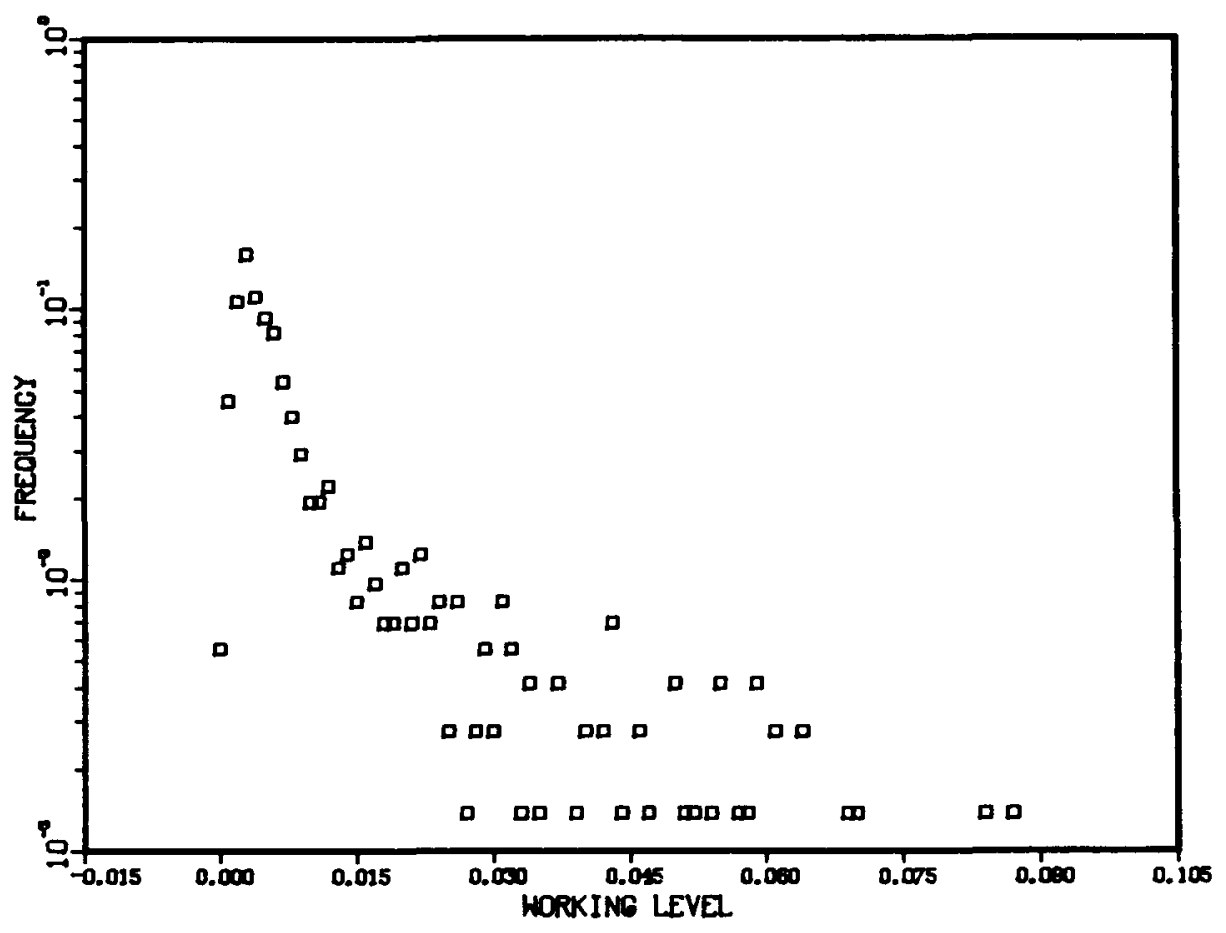

STATION 103, JUNE 1978

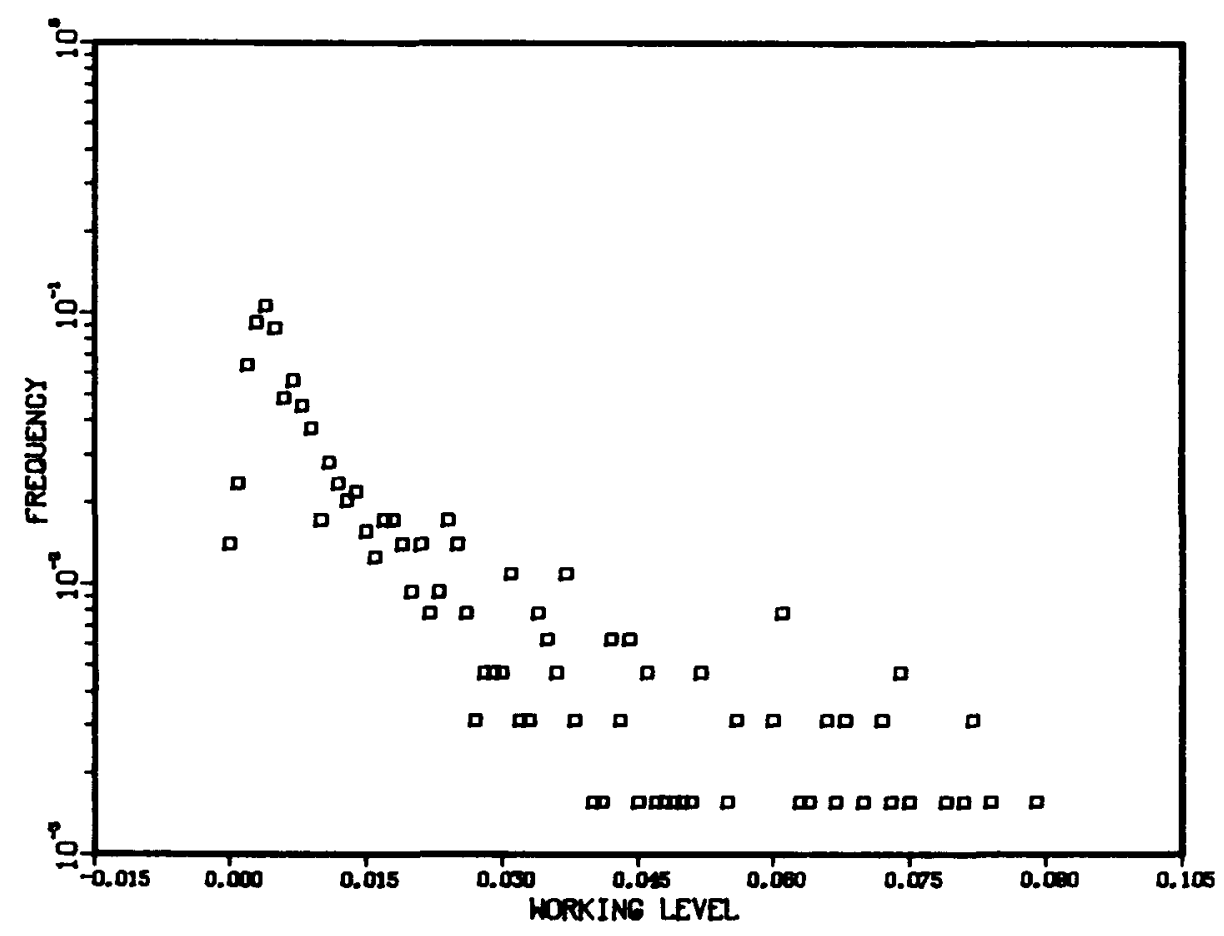

Figure Al5. Continued. 
STATION 103, JULY 1978

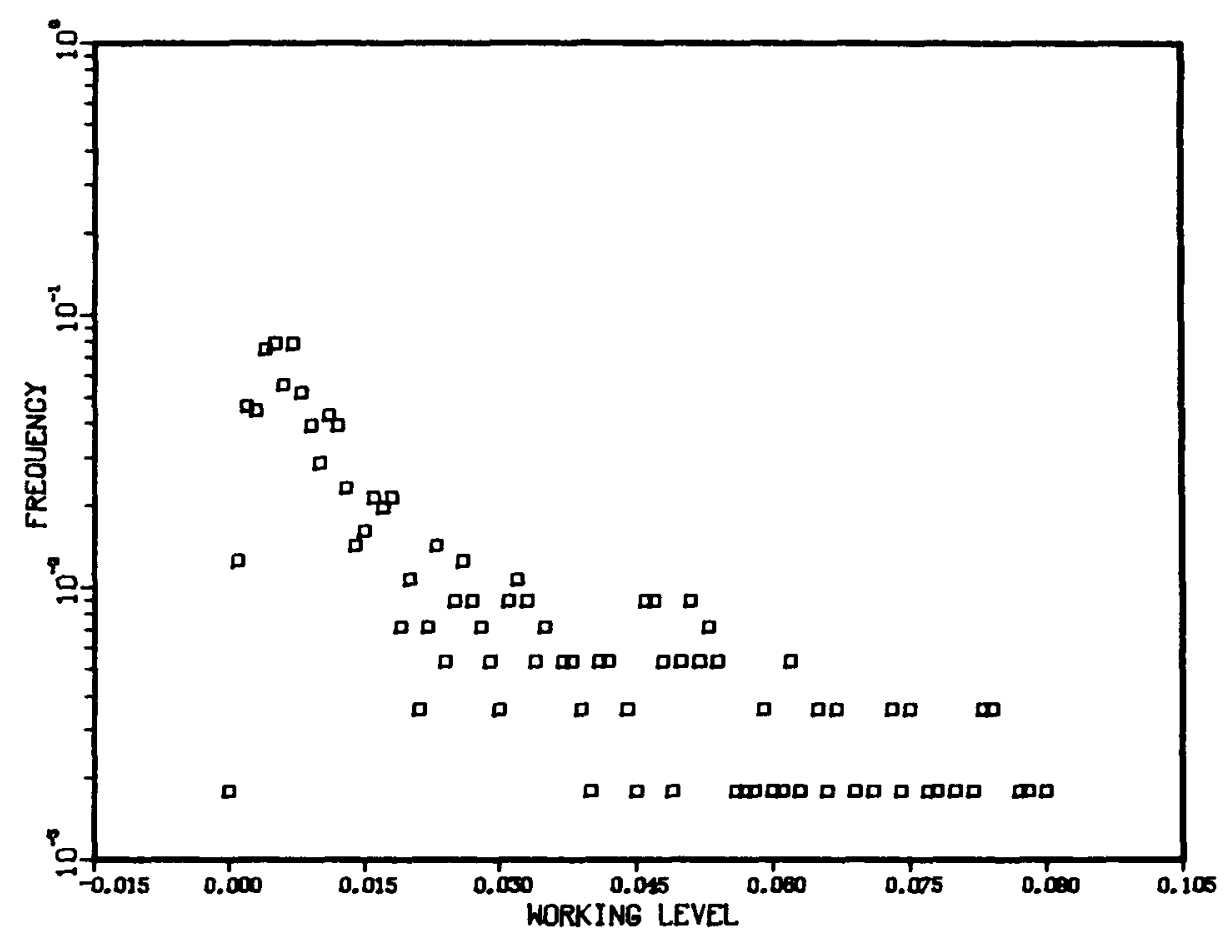

Figure A15. Continued. 
STATION 104, JULY 1977

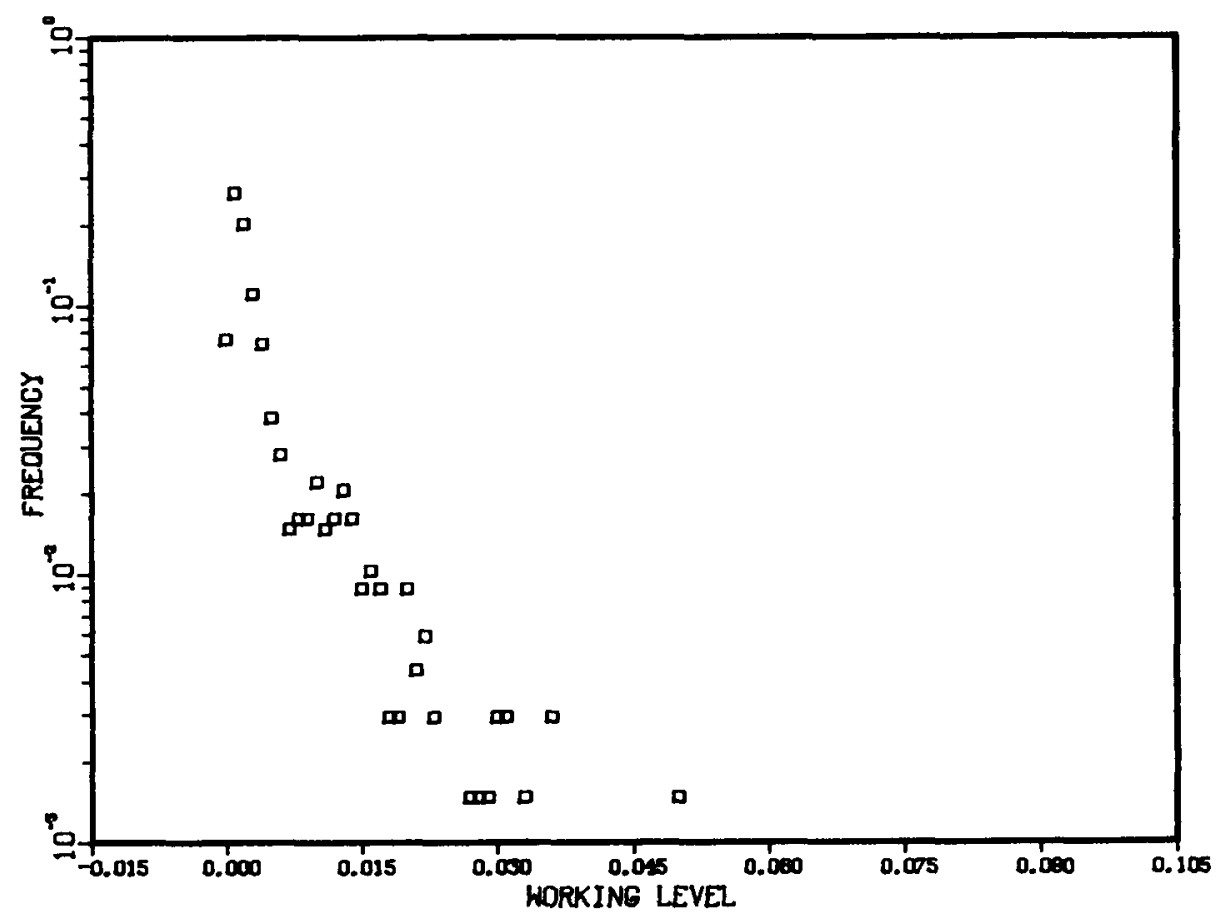

STATION 104, AUGUST 1977

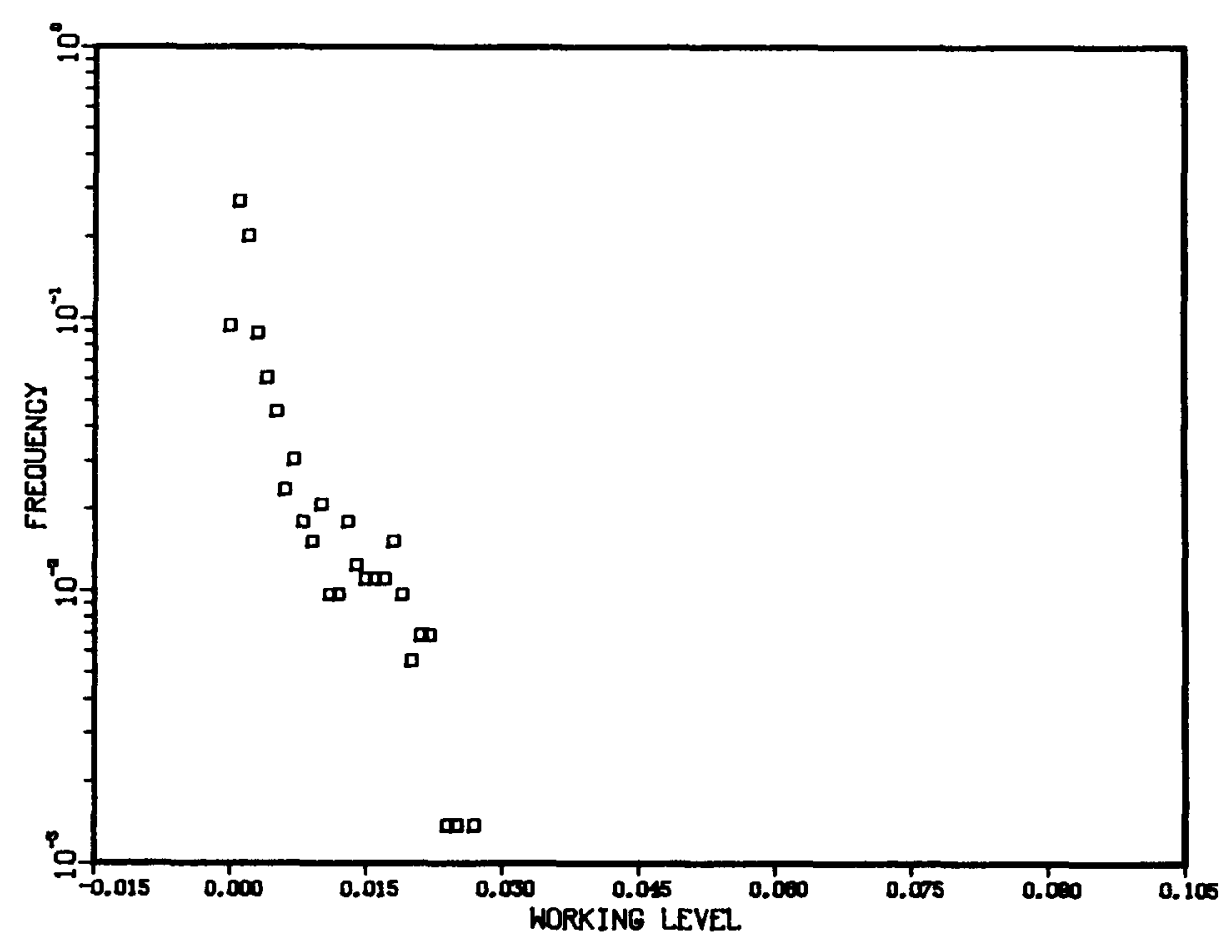

Figure A16. Working-Level Frequency at Station 104. 
STATION 104, SEPTEMBER 1977

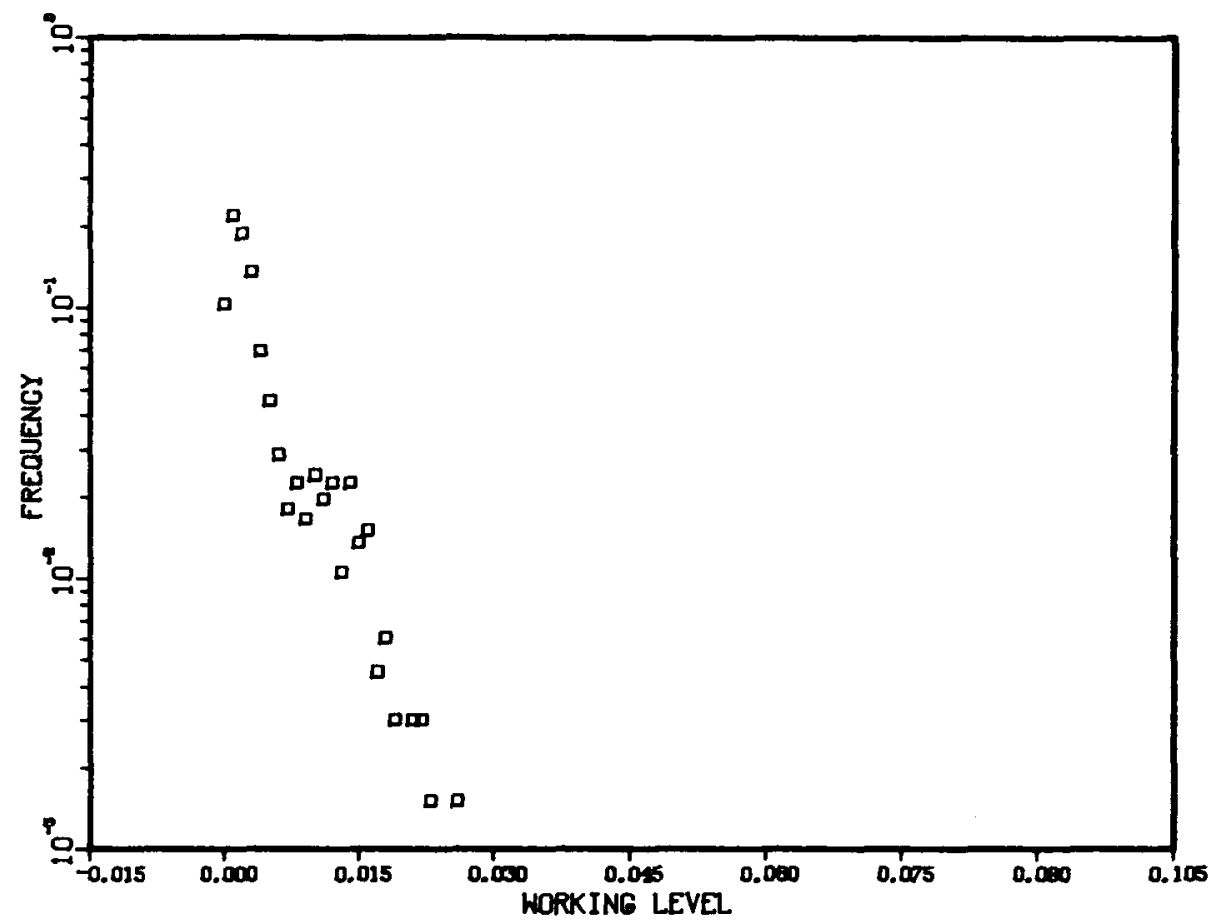

STATION 104, OCTOBER 1977

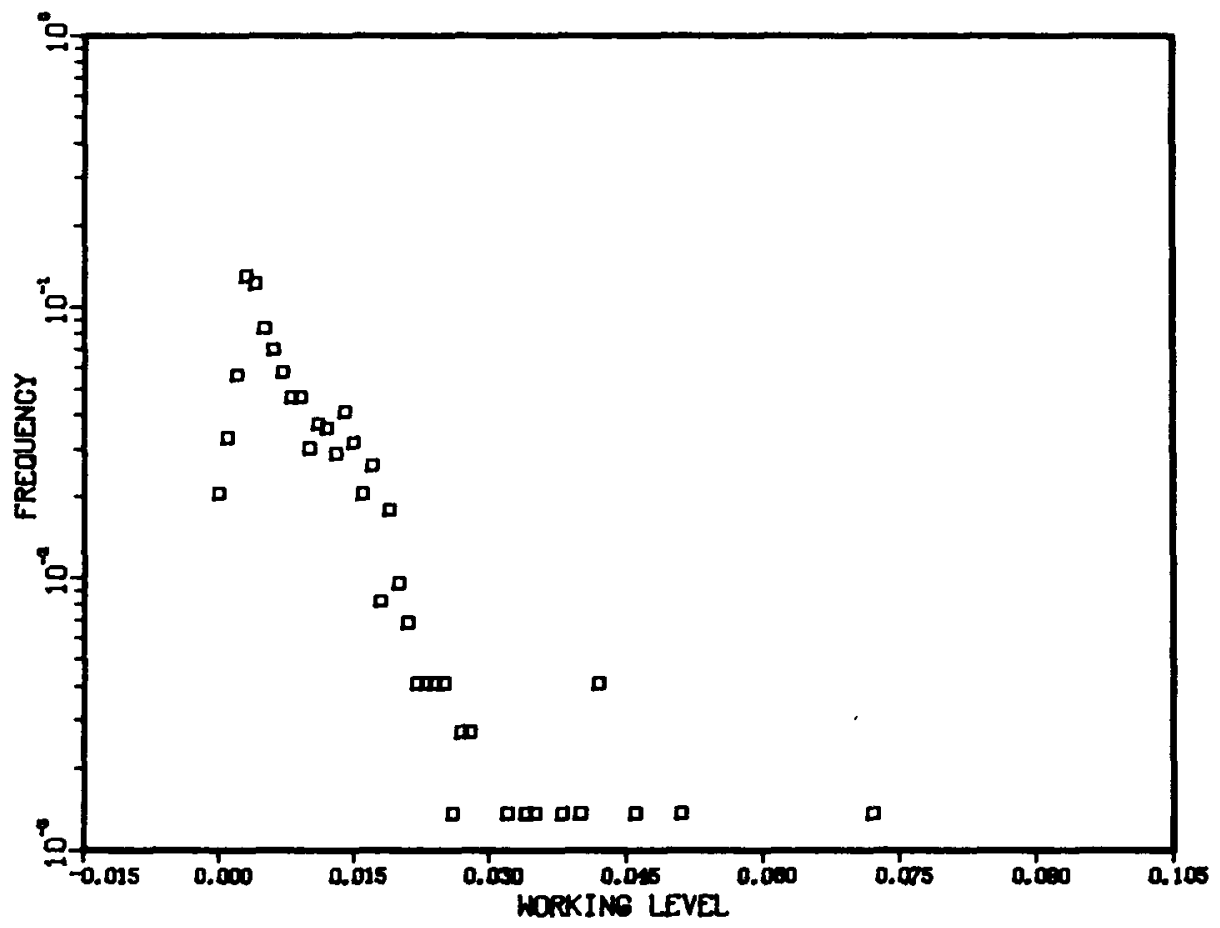

Figure Al6. Continued. 
STATION 104, DECEMBER 1977

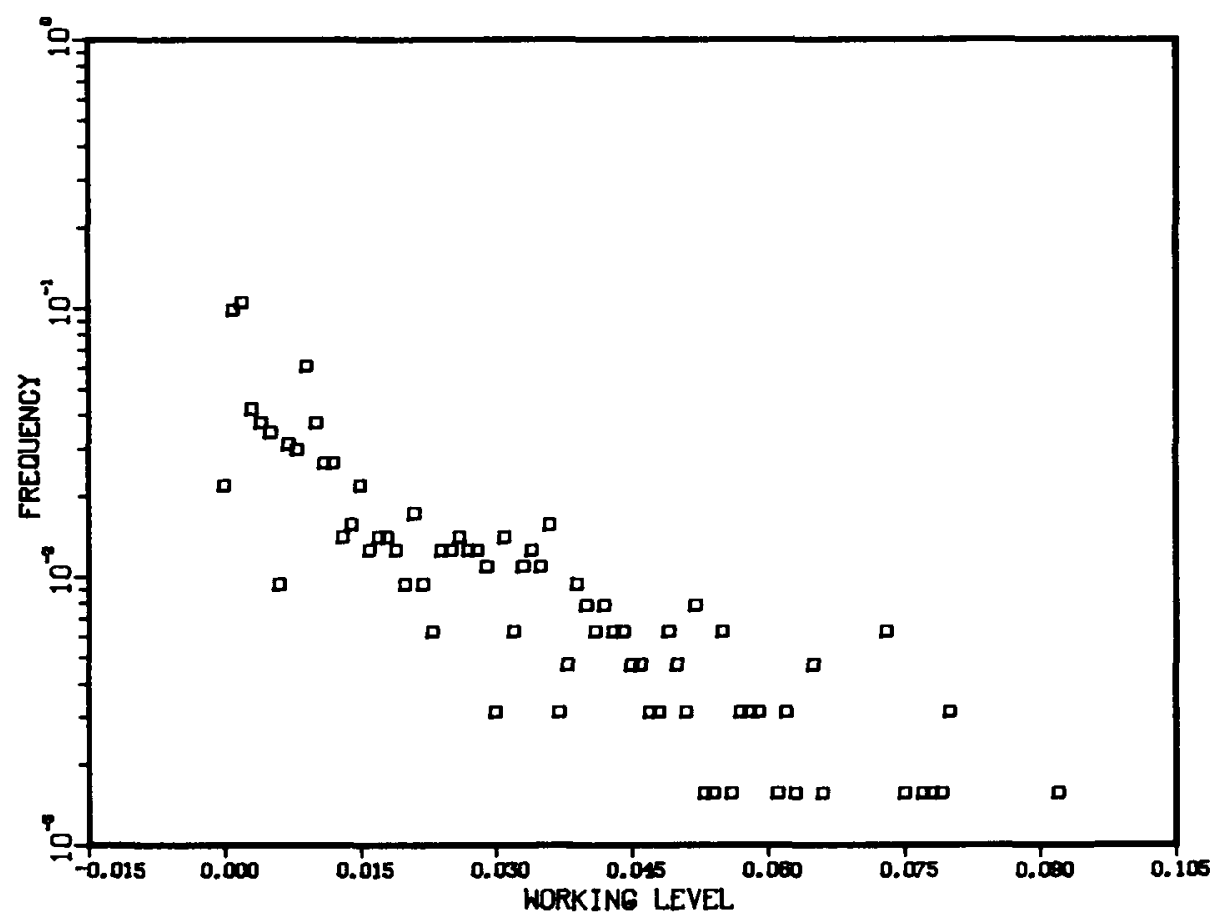

STATION 104, JANURRY 1978

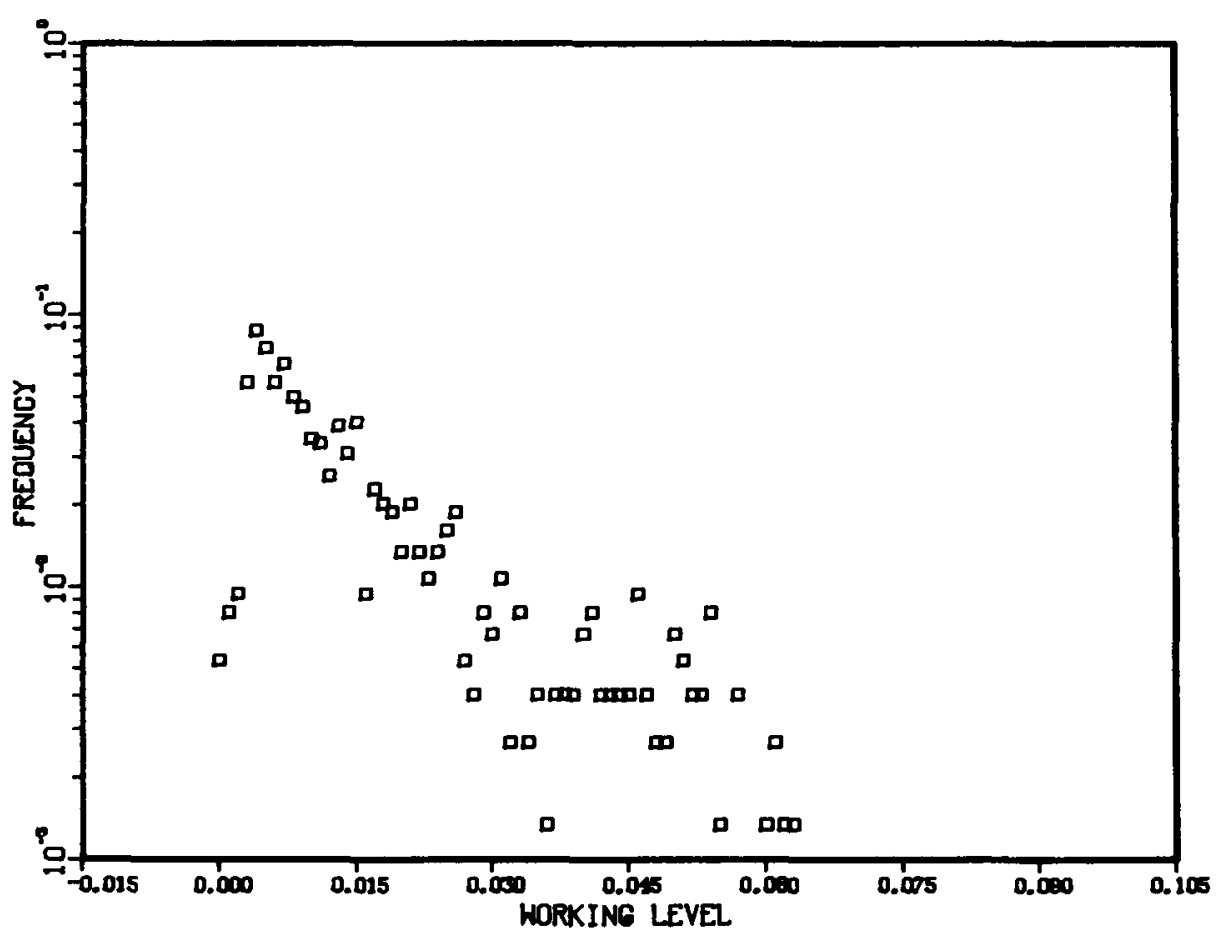

Figure Al6. Continued. 
Distribution of

NUREG/CR-1133 Appendix (ANL/ES-81 Appendix)

Internal:

A.J. Dvorak

P.F. Gustafson

W.J. Hallett

R.B. Holtzman

W.E. Kisieleski (20)

P. Frenzen

C.E. Dungey

J.B. Lindstrom

G.L. Montet
P. Chee

N. Kretz

M.H. Momeni (110)

C.J. Roberts

J. Rundo

W.K. Sinclair

J.H. Kittel

R.B. Keener

J.H. Martens
B. Hicks

D. Grahn

A.F. Stehney

Y.C. Yuan

A.J. Zielen

ANL Contract File

ANL Libraries (5)

TIS Files (6)

\section{External:}

NRC Washington, for distribution per RH (160)

Manager, Chicago Operations and Regional Office, DOE

Chief, Office of Patent Counsel, DOE-CORO

President, Argonne Universities Association, Argonne, IL

R.E. Alexander, USNRC, Washington

A.J. Breslin, Health and Safety Lab., USDOE, New York, NY 10014

A. Brodsky, USNRC, Washington

T. Buh1, State of New Mexico Health and Social Services Dept., P.0. Box 2536, Milan, NM 87021

R.E. Cunningham, USNRC, Silver Spring, MD 20910

R.S. Daniels, NUS Corp., 4 Research Place, Rockville, MD 20850

R.L. Doty, Tennessee Valley Authority, Muscle Shoals, AL 35660

R. Douglas, U.S. Environmental Protection Agency, P.0. Box 15027, Las Vegas, NV 89114

M.B. Emmett, Oak Ridge National Lab., Oak Ridge, TN 37830

W.C. Fort, U.S. Environmental Protection Agency, P.0. Box 15027, Las Vegas, NV 89114

W.E. Gray, Anaconda Co., 660 Bannock St., Denver, CO 80204

D.W. Hendricks, U.S. Environmental Protection Agency, P.0. Box 15027, Las Vegas, NV 89114

J. Kastner, USNRC, Washington

E.E. Kennedy, United Nuclear - Homestake Partners, P.0. Box 98, Grants, NM 87020

R.H. Kennedy, Div. Environmental Control Technology, USDOE, Washington

H. Lowenberg, USNRC, Washington

P. Magno, U.S. Environmental Protection Agency, 1921 Jefferson Davis Highway, Arlington, VA 22202

D. Martin, USNRC, Washington

J. Martin, U.S. Environmental Protection Agency, 1921 Jefferson Davis Highway, Arlington, VA 22202

J. Martin, USNRC, Washington

C. Meyers, Tennessee Valley Authority, 100 Union Bldg., Knoxville, TN 37902

H.J. Miller, USNRC, Washington

W. Mills, U.S. Environmental Protection Agency, 1921 Jefferson Davis Highway, Arlington, VA 22202

C. Nelson, U.S. Environmental Protection Agency, 1921 Jefferson Davis Highway, Arlington, VA 22202 
L. Santos, USNRC, Washington (50)

L.C. Schwendiman, Battelle Pacific Northwest Lab., P.0. Box 999, Richland, WA 99352

M.B. Sears, Oak Ridge National Lab., Oak Ridge, TN 37830

G.A. Sehme1, Battelle Pacific Northwest Lab., P.0. Box 999, Richland, WA 99352

W.J. Shelley, Kerr-McGee Nuclear Corp., P.O. Box 25861, Oklahoma City, OK 73125

W. Thompson, USNRC, Washington

J.R. Travis, Oak Ridge National Lab., Oak Ridge, TN 37830

J. Watson, University of North Carolina, Chape1 Hi11, NC 27514

R. Wilde, USNRC, Washington

M.E. Wrenn, University of Utah, Salt Lake City, UT 84112

W. Zobel, Tennessee Valley Authority, 100 Union Bldg., Knoxville, TN 37902

DOE-TIC

(2) 